\title{
Regulatory mechanisms driving motor neuron functional diversification
}

\author{
Dissertation \\ for the award of the degree \\ "Doctor of Philosophy (Ph.D.)" \\ Division of Mathematics and Natural Sciences \\ of the Georg-August-Universität Göttingen \\ within the doctoral program of Biology \\ of the Georg-August University School of Science (GAUSS)
}

Submitted by

Mudassar Nazar Khan

from

Lodi, California USA

Göttingen, March $1^{\text {st }}, 2018$ 


\section{THESIS COMMITTEE MEMBERS}

\section{Committee Members:}

Prof. Dr. Till Marquardt (Supervisor, Reviewer)

Interfaculty Chair for Neurobiological Research

Clinic for Neurology, University Clinic Aachen

Prof. Dr. Ernst Wimmer (Reviewer)

Dept. of Developmental Biology

Johann-Friedrich-Blumenbach-Institute of Zoology and Anthropology, Göttingen

Prof. Dr. med. Hansjörg Scherberger

Research Group Neurobiology

German Primate Center (DPZ), Göttingen

\section{Extended Committee Members/Members of the Examination Board:}

Prof. Dr. Stefan Bonn

Institute of Medical Systems Biology

Center for Molecular Neurobiology Hamburg (ZMNH), University Clinic Eppendorf,

Hamburg

Dr. Camin Dean, Ph.D.

Trans-synaptic Signaling

European Neuroscience Institute (ENI), Göttingen

Prof. Dr. Stefan Jakobs

Dept. of NanoBiophotonics

Max Planck Institute for Biophysical Chemistry, Göttingen

Date of oral examination: April $24^{\text {th }}, 2018$ 
"What a piece of work is a man ... in form and moving how express and admirable! in action how like an angel..."

-Shakespeare, Hamlet 


\section{DECLARATION AND CONTRIBUTIONS}

I confirm that I composed the following Ph.D. thesis entitled "Regulatory mechanisms driving motor neuron functional diversification." I designed and performed the experiments and analyzed the results of the experiments with the exception of the indicated contributions by collaborators:

Prof. Dr. Till Marquardt, provided advice on the experimental design, data analysis and editorial comments on this work.

Dr. Tsung-I Lee, Ph.D. cloned the mouse Err2 and Err3 DNA constructs.

Dr. rer. nat. Piotr Fabrowski performed the ECR browser searches, cloned the KcnalO DNA construct and promoter constructs.

Dr. Camille Lancelin, Ph.D. performed the OSC-PLS analysis.

Ashish Rajput performed the chick spinal column RNA extraction and sequencing experiments

Dr. Vikas Bansal, Ph.D. performed the RNA sequencing analysis.

Mudassar Nazar Khan

Göttingen, March 1 $1^{\text {st }}, 2018$ 


\section{ACKNOWLEDGEMENTS}

I am profoundly grateful to my thesis supervisor Prof. Dr. Till Marquardt for his generous support, patience, immense knowledge and motivation in all facets of the Ph.D. process. I am grateful for your trust in my abilities as a student in your lab and I cherish your enthusiasm and guidance, which allowed me to grow as a student of science and will continue to shape my future studies. I also enjoyed the times discussing music and philosophy and telling funny stories and jokes.

I express my gratitude towards my thesis committee members for stimulating me to think deeper about my work and sharing their knowledge: Prof. Dr. Ernst Wimmer and Prof. Dr. Hansjörg Scherberger. I would like to thank the extended committee members, Prof. Dr. Stefan Bonn, Dr. Camin Dean and Prof. Dr. Stefan Jakobs for accepting to read my thesis work. And I would like to thank Prof. Dr. Dieter Heineke for his support and guidance in the GAUSS biology program at Universität Göttingen.

I would like to acknowledge my colleagues in the Marquardt lab-Thanks for sharing your expertise and your time with me and I enjoyed working you all: Yehan Bian, Pitchaiah Cherukuri, Piotr Fabrowski, Alexandra Klusowski, Jacqueline Kueh, Camille Lancelin, Daniel Müller, Chor Han Poh, Nidhi Subhashini, Beate Veith, Liang Wang.

I am indebted to Daniel Müller for all the questions he answered with patience and care. Your help during this process meant a lot to me. Thanks for sharing your knowledge, experience and expertise and a lot of laughs with me.

Also, I am indebted to Pitchaiah Cherukuri and Piotr Fabrowski, who were generous in sharing their knowledge, experience, expertise and a lot of laughs during my time in the laboratory.

For all the collaborators on the Err2/3 project: I especially want to thank Dr. Pitchaiah Cherukuri for technical support in electrophysiology studies and suction electrode recording collaboration, Dr. Piotr Fabrowski for cloning studies collaboration. Furthermore, I thank Dr. Camille Lancelin for her collaboration on PCA analysis for the mouse behavior studies and Yehan Bian collaboration for muscle spindle studies. I want to thank Dr. Stefan Bonn for the collaboration on chicken RNA sequencing and analysis. I thankful for and appreciate working with Ashish Rajput, Dr. Vikas Bansal, and Dr. Michel Edwar. I would like to thank Dr. Robert Brownstone and Dr. Isabelle Panek for helping me to learn how to perform electrophysiological recordings in mouse spinal cord slices, which was very important for 
this work. I would like to thank Alexandra Klusowski and Beate Veith for their general assistance in the lab.

I would like to thank European Neuroscience Institute (ENI) staff members for their support, especially Nils for microscopy help, Tim and Matthias for IT-Service help, Frank for behavioral apparatus help and Franzi, Jessica and Verena for animal husbandry. I would like to thank my students, Simeon and Saskia for invigorating the enthusiasm to teach and learn.

To my family: To my mother, I appreciate your enduring love and wishes, to my father, thanks for your patience and support and to my younger sister and two brothers, thank you for being my friends. To my grandmother and grandfather, thank you for the love, hope and wisdom that you have tried to instill in me. I dedicate this work to my biological father. You continue to live through me. To my family here in Germany, thanks for all the love and support.

I want to convey my deepest gratitude towards my beautiful wife: thank you for your enduring love, support, and vast patience. Thanks for being a part of my life.

Finally, I thank God-the origin of all forms of expression for the gift of life and opportunity to gain deep knowledge of this world and of myself. 


\section{ABSTRACT}

Precise neural control of muscle tone, reflexes, and voluntary movements relies on circuits operating through the fusimotor neurons. Fusimotor neurons or gamma motor neurons ( $\gamma$-MNs) control motor behavior by tuning the sensitivity of the muscle spindle (MS) stretch receptors. However, the molecular mechanisms that endow $\gamma$-MNs with unique functional properties that are different from the force-generating alpha motor $(\alpha-\mathrm{MNs})$ are largely unknown. Therefore, the role of estrogen-related receptors Err2 and Err3 in specifying $\gamma$-MN biophysical properties was determined using chick and mouse models. I hypothesized that Err2 and Err3 are functionally redundant transcription factors that regulate $\mathrm{MN}$ functional diversification by promoting $\gamma$-MN biophysical properties. To test this hypothesis, quantitative immunodetection studies were performed on Err2 and Err3 expression during early postnatal development in mice. Forced expression of Err2 and Err3 was achieved longterm in chick MNs through a Tol2 transposon-mediated gene transfer strategy, which was followed by whole cell patch-clamp recordings to determine their effects on MN biophysical properties. Furthermore, conditional knockout mice lacking Err2 and Err3 $\left(E r r 2 / 3^{c K O}\right)$ in $\gamma$ MNs were generated. Err $2 / 3^{c K O} \mathrm{MNs}$ were recorded using whole cell patch-clamp and their motor behaviors were analyzed, including gait and precision movements. Lastly, RNA screening studies, reporter gene studies and whole cell patch-clamp recordings in chick were used to identify Err2/3 target genes. Based on data from these studies, I concluded that Err2 and Err3 are important for functional diversification of MNs into $\gamma$ - and $\alpha-\mathrm{MNs}$ and the acquisition of $\gamma-\mathrm{MN}$ biophysical properties that are required for accurate motor behaviors in mice. 


\section{LIST OF ABBREVIATIONS \& SYMBOLS}

\begin{tabular}{|c|c|c|c|}
\hline$\%$ & Percent & $\mathbf{M}$ & Molar \\
\hline${ }^{\circ} \mathbf{C}$ & Degrees Celsius & $\mathbf{m}$ & Milli \\
\hline $\mathbf{A}$ & Ampere & $\mu \mathbf{m}$ & $\operatorname{Micron}(\mathrm{s})$ \\
\hline AHP & After-hyperpolarization & M $\Omega$ & Mega Ohm \\
\hline Bmps & Bone morphogenetic proteins & ms & Millisecond \\
\hline b.p. & Base pair & $\min$ & minutes \\
\hline BSA & Bovine Serum Albumin & MN & Motor neuron (s) \\
\hline cDNA & Complementary DNA & MS & Muscle spindle(s) \\
\hline cKO & Conditional knockout & $\mathbf{m V}$ & Millivolt(s) \\
\hline ChAT & Choline acetyltransferase & $\mathbf{n}$ & \# of neurons \\
\hline CNS & Central Nervous System & $\mathbf{N}$ & \# of animals \\
\hline $\operatorname{deg}$ & Degrees & NeuN & Neural Nuclei \\
\hline DEPC & Diethylpyrocarbonate & O.C.T. & Optimal cutting temperature \\
\hline DMSO & Dimethyl Sulfoxide & O.N. & Overnight \\
\hline $\mathbf{E}$ & Embryonic Day & $\mathbf{p}$ & $p$-value \\
\hline ECR & $\begin{array}{l}\text { Evolutionary conserved non- } \\
\text { coding regions }\end{array}$ & $\begin{array}{l}\mathbf{P} \\
\mathbf{p A}\end{array}$ & $\begin{array}{l}\text { Postnatal day } \\
\text { Picoampere }\end{array}$ \\
\hline EDTA & $\begin{array}{l}\text { Ethylene diaminetetraacetic } \\
\text { acid }\end{array}$ & $\begin{array}{l}\text { PBS } \\
\text { PCR }\end{array}$ & $\begin{array}{l}\text { Phosphate buffered saline } \\
\text { Polymerase chain reaction }\end{array}$ \\
\hline eGFP & Enhanced GFP & pMN & MN progenitor cells \\
\hline EGTA & Ethylene glycoltetraacetic acid & PGC & Preganglionic motor column \\
\hline Egr3 & Early growth response 3 & r.p.m & Revolutions per min \\
\hline EnR & Engrailed repressor domain & RA & Retinoic Acid \\
\hline Err2 & Estrogen-related receptor beta & RNA & Ribonucleic Acid \\
\hline $\begin{array}{l}\text { Err3 } \\
\text { gamma }\end{array}$ & Estrogen-related receptor & $\begin{array}{l}\text { PFA } \\
\text { SDS }\end{array}$ & $\begin{array}{l}\text { Paraformaldehyde } \\
\text { Sodium Dodecyl Sulfate }\end{array}$ \\
\hline FG & Fluoro-Gold & $\mathbf{s}$ & Second(s) \\
\hline FGF & Fibroblast growth factor & S.E.M. & Standard error of the mean \\
\hline Fig. & Figure & Shh & Sonic hedgehog \\
\hline g & gram & $\mathbf{V}$ & Ventral \\
\hline GDNF & $\begin{array}{l}\text { Glia cell line derived } \\
\text { neurotrophic factor }\end{array}$ & vAChT & $\begin{array}{l}\text { Vesicular acetylcholine } \\
\text { transporter }\end{array}$ \\
\hline GFP & Green Fluorescent Protein & vGlut1 & Vesicular glutamate \\
\hline GTOs & Golgi tendon organs & & transporter 1 \\
\hline h & Hours & VP16 & Herpesvirus protein VP16 \\
\hline HB9 & MN and pancreas homeobox & Wnt & Wingless-related MMTV \\
\hline H-H & Hamburger-Hamilton & $\mathbf{w} / \mathbf{v}$ & Weight per volume \\
\hline HMC & Hypaxial motor column & $\mathbf{x} \mathbf{g}$ & gravitational acceleration \\
\hline Hox & Homeobox & & $(9.81)$ \\
\hline IRES & Internal ribosome entry site & & \\
\hline K.O. & knockout & & \\
\hline $\mathbf{K b}$ & Kilo base pair & & \\
\hline Isl-1 & Islet-1 & & \\
\hline 1 & Liter & & \\
\hline Lhx & LIM homeobox protein & & \\
\hline LMC & Lateral motor column & & \\
\hline MMC & Medial motor column & & \\
\hline
\end{tabular}




\section{TABLE OF CONTENTS}

I. DECLARATION AND CONTRIBUTIONS 4

II. ACKNOWLEDGEMENTS 5

III. ABSTRACT

IV. LIST OF ABBREVIATIONS AND SYMBOLS 8

V. TABLE OF CONTENTS 9

VI. LIST OF FIGURES 13

VII. LIST OF TABLES 16

1. INTRODUCTION

1.1 Generation of motor neuron (MN) diversity in the embryonic spinal cord 18

1.1.1 Embryogenesis, neurulation and the generation of MNs 18

1.1.2 Refinement of MN identity based on columnar position and peripheral axonal projection 22

1.1.3 Refinement of MN subtype identity based on motor "pool" position 24

1.2 Proprioception and motor control 25

1.3 The muscle spindle (MS) apparatus 26

1.3.1 MS morphogenesis 27

1.3.2 The role of $\gamma$-MNs in the physiological function of the MS 28

1.3.2.1 $\alpha-\gamma$ co-activation without $\gamma$-MN input ___ 29

1.3.2.2 The stretch reflex__ 30

1.3.2.3 Beta motor neurons $(\beta-\mathrm{MNs}) \_31$

1.4 MN functional diversification_____ 31

1.4.1 $\alpha$ - and $\gamma$-MN Ia afferent connectivity and morphology ___ 32

1.4.2 Molecular correlates distinguishing $\alpha$ - and $\gamma-\mathrm{MNs}$

1.4.2.1 Molecular differences among $\alpha$-MN subtypes: fast $\alpha$-MNs and slow $\alpha-M N s$

1.4.3 MN biophysical properties ___ 34

1.4.3.1 Biophysical properties of $\alpha-\mathrm{MN}$ innervating specific motor units in cat and rat___ 35

1.4.3.2 Characterization of $\alpha$ - and $\gamma$-MN biophysical properties in cat___ 36

$1.5 \quad$ Nuclear receptors (NRs) _ 39

1.5.1 Estrogen-related receptor (ERR) family structural elements and phylogeny ___ 40

1.5.1.1 Estrogen-related receptor 1 (Err1)____ 41

1.5.1.2 Estrogen-related receptor $2($ Err2)____ 41

1.5.1.3 Estrogen-related receptor $3($ Err3)_____ 42

1.6 Rationale and Objectives ___ 42 
2. MATERIALS \& METHODS

2.1 Laboratory consumables and glassware/plasticware 44

2.2 Antibodies 44

2.3 Chemicals 44

$2.4 \quad$ Enzymes 47

$2.5 \quad$ Kits 47

2.6 Plasmids 47

2.7 Programs \& Databases ___ 48

$2.8 \quad$ Solutions ___ 49

2.9 Animal care ___ 49

2.10 Mouse interbreeding and mouse genotyping ___ 49

2.10.1.1 Mouse genotyping__ 52

2.10.1.1.1 Genotyping Chat::Tomato mouse line __ 53

2.10.1.1.2 Genotyping of Esrrb-Chat-Cre knockout mouse line___ 55

2.10.1.2 Genotyping of Esrrg-Chat-Cre knockout mouse line___ 55

2.11 Mouse behavior experiments ___ 56

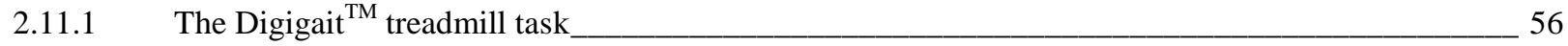

2.11.1.1 Orthogonal Signal Correction Partial Least Squares (OSC-PLS) analysis _ 58

2.11.2 The horizontal ladder task ___ 61

2.11.3 The horizontal beam task___ 62

2.12 Quantitative immunodetection studies ___ 63

2.12.1 Chick tissue processing ___ 63

2.12.2 Chick quantitative immunodetection studies ___ 63

2.12.3 Fluoro-Gold (FG) MN labeling experiments___ 63

2.12.4 Mouse tissue processing____ 64

2.12.5 Mouse quantitative immunodetection studies __ 64

2.12.6 Chick and mouse confocal imaging and quantification ___ 64

2.12.7 Quantification of vGlut1 synaptic varicosities using Imaris software ___ 65

$\mathbf{2 . 1 3}$ In ovo electroporation experiments ___ 65

2.13.1 Tol2 transposon system: stable in ovo transfection of chick MNs ___ 66

2.13.2 Chick Kcna10 DNA construct ___ 68

2.13.3 Mouse Kcnalo enhancer DNA constructs_____ 69

2.13.4 Chick RNA isolation and sequencing ___ 70

2.13.5 Chick RNA sequencing analysis ___ 72

2.14 Chick and mouse electrophysiology ___ 72

2.14.1 Chick spinal cord slice preparation ___ 72

2.14.2 Chick whole cell patch-clamp recordings ___ 72 
2.14.3 Mouse spinal cord slice preparation 74

2.14.4 Mouse whole cell patch-clamp recordings___ 74

2.14.5 Chick and Mouse whole cell patch-clamp analysis ___ 75

2.15 Bacterial transformation and DNA extraction

2.16 Statistical analysis ___

3. RESULTS 80

3.1 Characterization of Err2 and Err3 transcription factor expression pattern in wild-type mouse spinal cord 80

3.1.1 Err2 and Err3 expression pattern during early postnatal development 80

3.2 Err2 and Err3 expression pattern in $E r r 2 / 3^{c K O}$ mouse spinal cord 83

3.2.1 Characterization of putative $\gamma$-MN connectivity in $E r r 2 / 3^{c K O}$ mice 86

3.3 Characterization of MN biophysical properties in $E r r 2 / 3^{c K O}$ mice 89

3.3.1 Characterization of putative $\alpha$ - and $\gamma$-MNs biophysical properties in control versus Err $2 / 3^{c K O}$ mice 90

3.4 Characterization of gait patterns and precision movements during locomotion in Err2/3 ${ }^{\text {cKO }}$ mice 94

3.4.1 Characterization of gait in Err $2 / 3^{c K O}$ mice using the DigiGait ${ }^{\mathrm{TM}}$ treadmill task ___ 95

3.4.2 The horizontal ladder task____ 98

3.4.3 The horizontal beam task___ 99

3.5 Err2 and Err3 function in driving chick MN biophysical properties ___ 100

3.6 The mechanistic role of Err2 in the influence of $\gamma$-MN biophysical properties___ 104

3.6.1 Err2 up-regulates Kcna10 gene expression____ 104

3.6.2 Err2 directly regulates Kcnal0 gene activity ___ 106

3.6.3 Kena10 biophysical properties in chick MNs ___ 108

4. DISCUSSION

4.1 Molecular characteristics of $\gamma$-MNs 111

4.2 Distinct biophysical properties of $\alpha$ - and $\gamma$-MNs in mice 113

4.3 The role of Err2 and Err3 in driving $\gamma$-MN biophysical properties 113

4.4 The role of Err2 and Err3 in determining mouse gait and precision movements 114

4.5 The role of Err2 and Err3 in driving a $\gamma$-MN biophysical signature 115

4.6 Working towards a molecular mechanism that generates $\alpha$ - and $\gamma$-MN functional diversification 116

4.7 Err2 and Err3 actions specifically promote $\gamma$-MN function but not identity 117

4.8 Outlook: The role of Err2 and Err3 in extraocular MN functional properties and proprioception 
5. SUMMARY 121

6. REFERENCES 123

7. APPENDIX 1 141

8. APPENDIX 2 144

9. APPENDIX 3 148

10. CURRICULUM VITAE 152 


\section{LIST OF FIGURES}

Figure 1.1 Early depiction of neural diversity: Santiago Ramón y Cajal's schematic of the cellular

nature of the nervous system 18

Figure 1.2 The development of the embryonic spinal cord 19

Figure 1.3 The dorsoventral (d-v) axis patterning 20

Figure 1.4 Ventral neural progenitor cell identity and MN cell fate 21

Figure 1.5 Schematic representation of the MN columns within the rostrocaudal (r-c) axis 23

Figure 1.6 Hox genes regulate motor column specific innervations 24

Figure 1.7 Schematic depicting different types of MNs and their innervations 27

Figure 1.8 Schematic representation of $\alpha-\gamma$ co-activation 30

Figure 1.9 General patterns of properties in different MN subtypes 38

Figure 1.10 Schematic representation of nuclear receptor (NR) structure, group III nuclear receptor (NR3) phylogenetic tree and representation of estrogen-related receptor (ERR) structural elements 41

Figure 2.1 Schematic diagram of Chat ${ }^{\mathrm{Cre}}$ mouse line 50

Figure 2.2 The interbreeding strategy for generating Chat::Tomato mice 51

Figure 2.3 The interbreeding strategy for generating Esrrb ${ }^{\text {flox/flox }}$; Esrrg ${ }^{\text {flox/flox }}$; Chat ${ }^{\text {Cre }}$ conditional $^{\text {. }}$ knockout $\left(\right.$ Err2/3 $\left.{ }^{\mathrm{cKO}}\right)$ mice lacking Err2 and Err3 in MNs 52

Figure 2.4 Schematic diagrams showing digital paw print (Paw Contact Area) and gait signals for the forelimbs and hindlimbs 58

Figure 2.5 MN development in chick spinal cord 67

Figure 2.6 Stable transfection of chick MNs in the ventral horn of the spinal cord followed by whole cell patch-clamp recordings

Figure 2.7 Different combinations of DNA constructs electroporated in the chick neural tube for the Kcna10 ECR activity experiments

Figure 2.8 Different combinations of DNA constructs co-electroporated in the chick neural tube for the chick RNA sequencing studies

Figure 2.9 DNA constructs electroporated in chick neural tube for chick the whole cell patch-clamp recordings 73

Figure $2.10 \mathrm{MN}, \mathrm{SN}$ and MS differentiation in mice 75

Figure 2.11 F-I traces for calculating firing frequency and gain 76

Figure 2.12 Calculation of gain and firing frequency from F-I curve 77

Figure 3.1 Co-expression of high levels of Err2 and Err3 in relatively small soma size putative $\gamma$-MNs that express low levels of NeuN and incorporate high levels of FG at P21 81

Figure 3.2 High levels of Err2 and Err3 expression becomes more restricted to relatively small soma size MNs that express low levels of NeuN and incorporate high levels of FG at P21 82 
Figure 3.3 High expression levels of Err2 and Err3 distinguish relatively small soma size putative $\gamma$-MNs as development proceeds 83

Figure 3.4 Elimination of Err2 and Err3 expression from MNs in Err2/3 ${ }^{\mathrm{cKO}}$ mice 85

Figure 3.5 Lack of Err2 and Err3 relative expression levels in the putative $\gamma$-MNs from Err2/3 ${ }^{\mathrm{cKO}}$ mice compared to control mice 86

Figure 3.6 Visualization of Ia sensory synaptic termini enriched in vGlut $1^{+}$synaptic varicosities 87

Figure 3.7 Ia sensory afferent connectivty is not altered in Err2/3 ${ }^{\mathrm{cKO}}$ mice 88

Figure 3.8 Comparison of FG intensity and soma size to target $\gamma$-MNs in P21 W.T. mice for recordings 89

Figure 3.9 The loss of Err2 and Err3 in the putative $\gamma$-MNs from Err $2 / 3^{\mathrm{cKO}}$ mice results in shifted biophysical properties towards putative $\alpha-\mathrm{MNs}$

Figure 3.10 A putative $\gamma$-MN from Err2/3 ${ }^{\mathrm{cKO}}$ mice loses its biophysical signature and shifts towards $\alpha-\mathrm{MN}$ biophysical properties 92

Figure 3.11 The putative $\gamma$-MNs from Err2/3 ${ }^{\mathrm{cKO}}$ lose their biophysical signature and shift their biophysical properties towards $\alpha$-MNs 93

Figure 3.12 The putative $\alpha$-MNs from Err2/3 $3^{\mathrm{cKO}}$ do not shift their biophysical properties 94

Figure $3.13 \mathrm{Err} 2 / 3^{\mathrm{cKO}}$ mice have a different gait pattern in the forelimbs and the hindlimbs when compared to control mice 96

Figure 3.14 Specific gait variables predicted in distinguishing control versus Err2/3 ${ }^{\mathrm{cKO}}$ mouse gait patterns 97

Figure 3.15 Err2/3 ${ }^{\mathrm{cKO}}$ mice show deficits in precision movements when walking across a horizontal ladder 98

Figure 3.16 Err2/3 ${ }^{\mathrm{cKO}}$ show precision movement deficits when challenged with horizontal beam task 99

Figure 3.17 Forced expression of DNA constructs in MNs shifts their mean firing frequency 101

Figure 3.18 Forced expression of Err2VP16 in chick MNs drives their biophysical properties towards lower rheobase, higher firing frequency and gain 102

Figure 3.19 Forced expression of Err2VP16 in chick MN leads to a pattern of significantly higher firing frequency and higher gain

Figure 3.20 Forced expression of Err2VP16 in chick MNs drives their biophysical properties towards lower rheobase, higher firing frequency and gain 104

Figure 3.21 Forced expression of CMV-VP16-Err2-eGFP up-regulates Kcna10 RNA in chick spinal columns at E12.5 (HH St. 38-39) 105

Figure 3.22 Schematic of Kcna10 genomic locus showing the putative Err2/3 bindings sites in the Kcna10 ECR 106

Figure 3.23 Err2 regulates chick Kcna10 gene activity in MNs 107

Figure 3.24 Forced expression of Err2VP16 increases Kcna10 gene activity 108

Figure 3.25 Forced Kcna10 expression in chick MNs promotes $\gamma$-MN biophysical signature 109 
Figure 3.26 Forced expression of Kcna10 in chick MNs to a shift in biophysical properties towards lower rheobase, higher firing frequency, and higher gain 110

Figure 4.1 Err2 and Err3 expression in oculomotor nucleus of $\mathrm{ChAT}^{\text {Cre }}$; Rosa26 ${ }^{\text {floxtdTomato }}$ mice at three weeks of age postnatally

Figure 7.1 Anti-Err2 and anti-Err3 antibodies specifically recognize Err2 and Err3 proteins, respectively

Figure 7.2 Secondary antibodies $\operatorname{IgG} 2 \mathrm{~b}$ and IgG2a are isotype-specific for Err2 and Err3, respectively 


\section{LIST OF TABLES}

PAGE

Table 2.1 Primary Antibodies 44

Table 2.2 Secondary Antibodies 44

Table 2.3 Chemicals and Supplier 44

Table 2.4 Enzymes and Supplier 47

Table 2.5 Kits and Supplier 47

Table 2.6 Plasmids 47

Table 2.7 Software and Supplier 48

Table 2.8 Solutions 49

Table 2.9 Defined variables for forelimbs and hindlimb 60

Table 8.1 Summary of biophysical properties of putative $\alpha$ - and $\gamma$-MNs from 144

Table 8.2 Summary of biophysical properties of chick spinal cord MNs 146

Table 9.1 Forelimb variables control versus Err2/3 ${ }^{\mathrm{cKO}}$ at mice at all speeds 148

Table 9.2 Hindlimb variables control versus Err $2 / 3^{\mathrm{cKO}}$ at mice at all speeds 150

Table 9.3 $\mathrm{Q}^{2}$ and RMSEP p-value for forelimbs and hindlimbs 151 


\section{INTRODUCTION}

The central nervous system (CNS) has evolved in vertebrates and invertebrates so they can sense changes in their environment, process and integrate these changes and respond by performing specific actions or motor behaviors. In animals, automatic motor behaviors or locomotion is generated by neural networks in the spinal cord, however, the special capacity to perform precision tasks such as voluntary locomotion and manipulation of objects requires a constant flow of sensory information from the periphery. Therefore, this information from the nervous system must be integrated by spinal motor neurons (MNs), which generate and control muscular movements.

MNs within the spinal cord display diversity to adapt motor behaviors as vertebrates evolved from an aquatic to a terrestrial environment. The diverse classes and sub-classes of spinal MNs help the organism to move and control movement precisely. This poses several questions regarding MNs at various developmental and functional levels: first, how is the diversity of MNs in the embryonic and postnatal spinal cord acquired? second, how do spinal MN subtypes differ? third, what are the molecules that regulate the biophysical properties of MN subtypes and their function in locomotion?

This study aimed to identify molecular mechanisms that promote MN functional diversification, which permits the control of movement to enable tetrapod organisms to navigate and survive in their environment. Orphan nuclear receptors (ONRs) Err2 and Err3 were evaluated in the generation of specific biophysical membrane properties that enable specialized MNs called $\gamma$-MNs to function in circuits involved in coordination of limb movements or gait, maintenance of posture and execution of precision movements during locomotion in mice. Therefore, the lessons learned through these efforts may stimulate new studies that probe neural diversity tuned for function in other parts of the nervous system and may provide therapeutic strategies against neurodegenerative diseases linked to specific cell types. 


\subsection{Generation of motor neuron (MN) diversity in the embryonic spinal cord}

"True, it would be very convenient and very economical from the point of view of analytical effort if all the nerve centres were made up of a continuous intermediary network between the motor nerves and the sensitive and sensory nerves. Unfortunately, nature seems unaware of our intellectual need for convenience and unity, and very often takes delight in complication and diversity." -Santiago Ramón y Cajal, (Nobel Prize Lecture, 1906)

The field of neurobiology emerged when Santiago Ramón y Cajal, using the Golgi stain histological method, observed that the nervous system is organized into distinct units, neurons, that have diverse appearance; a finding that stimulated research into neural diversity (Fig. 1.1) (Masland, 2004). Since this initial discovery, many diverse neural cell classes and sub-classes have been identified in the nervous system mostly based on their appearance, sometimes on their biophysical properties and progressively on the genes or proteins they express (Masland, 2004). Diverse cell types emerge in the embryonic spinal cord during development, including MNs that further diversify subtype identities and emanate axons peripherally to target specific muscle groups (Jessell, 2000).
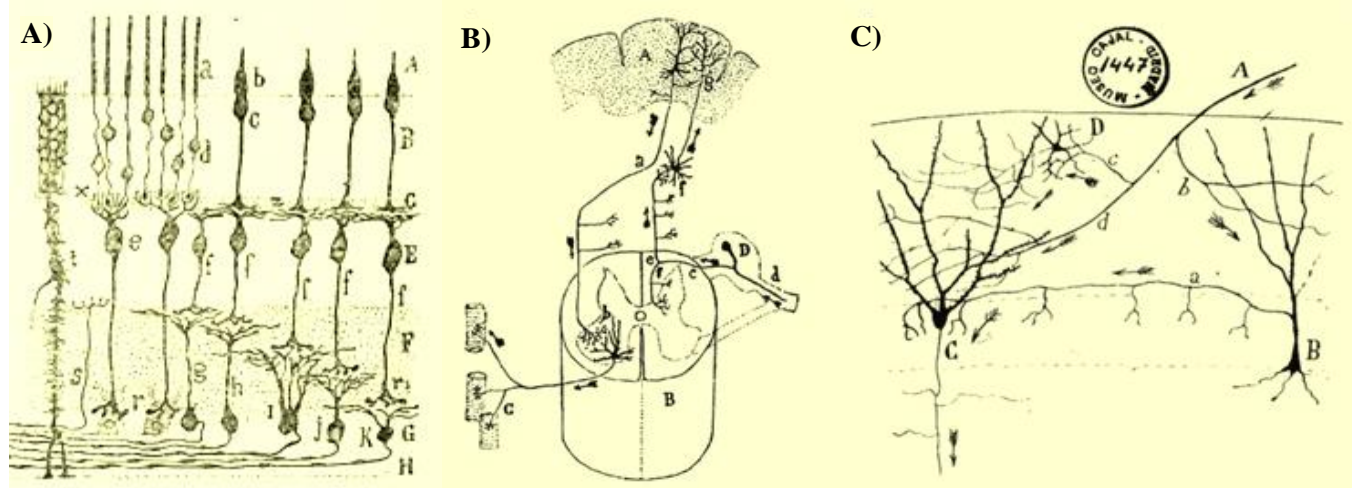

Figure 1.1 Early depiction of neural diversity: Santiago Ramón y Cajal's schematic of the cellular nature of the nervous system

A) Several cellular subtypes in the different levels of the retina. B) Long-range axon neurons of the cortex and the spinal cord. C) Short-range axon neurons of the hippocampus. (Adapted from Underwood, 2015 and Delgado-Garcia, 2015).

\subsubsection{Embryogenesis, neurulation and the generation of MNs}

Embryonic neural diversity produced in the nervous system during neurogenesis stems from cellular gene expression (cell lineage-rooted mechanism) and cellular communication (induction and cell-cell interaction grounded mechanism) (McConnell, 1995). During gastrulation, the chordamesoderm induces neural "fate" in the overlaying ectoderm that becomes the neural plate, a process known as neural induction. During neurulation, the notochord (forming from the chordamesoderm) induces ventral structure, while the dorsal 
lips of the neural plates fold inwards to generate the neural tube (Fig. 1.2B, C). In the neural tube, the neural progenitor cells proliferate and eventually generate neurons, astrocytes, and oligodendrocytes (Fig. 1.2D) (Purves, 2004).

A) Neural plate

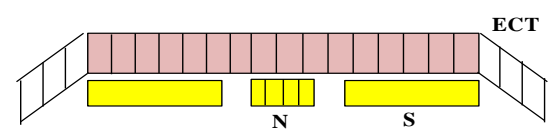

C) Neural tube
B) Neural fold

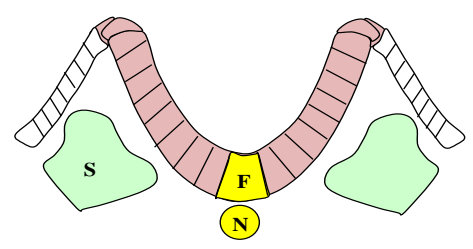

D) Spinal cord

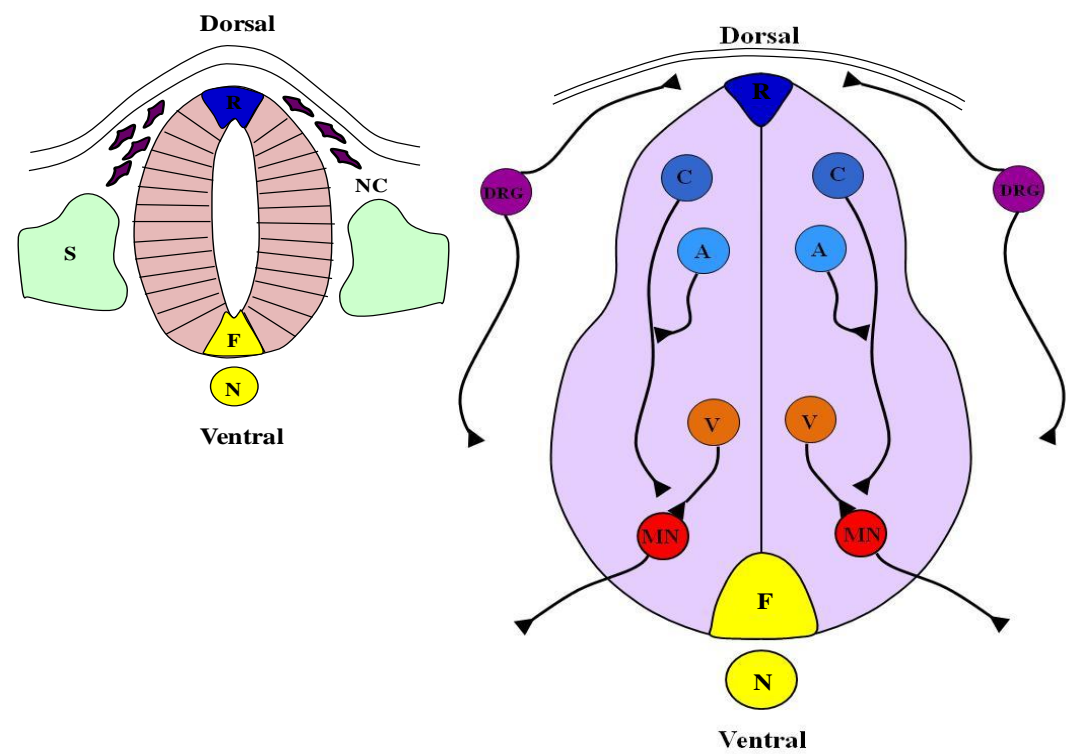

\section{Figure 1.2 The development of the embryonic spinal cord}

The development of the embryonic spinal cord consists of four stages: A) The neural plate stage: the neural cells have epidermal ectoderm (ECT) on their edge, while the notochord cells (N) are positioned centrally in the neural plate and the segmental plate mesoderm $(\mathrm{S})$ is positioned laterally in the neural plate. B) The neural fold stage: during neurulation, the floor plate (F) cells are observed in the ventral midline regions and the somatic mesoderm begins to take shape. $\mathrm{C}$ ) The neural tube stage: as the neural tube forms, the roof plate cells (R) differentiate and while the neural crest cells (NCCs) delaminate. D) The spinal cord: as development proceeds, the spinal cord develops diverse neural classes arrayed across a dorsoventral (d-v) axis: motor neuron (MN) and ventral interneurons (V) differentiate in the ventral region of the spinal cord while dorsal root ganglion neurons (DRGs), Commisural (C) neurons and Association neurons (A) differentiate in the dorsal region of the spinal cord. (Modified from Jessell, 2000; Placzek et al., 1991)

Within the neural tube, inductive signaling establishes neural class identities across two major spatial axes: 1) the dorsoventral (d-v) axis and 2) the rostrocaudal (r-c) axis (Jessell, 2000). As development proceeds, a latticed network of neural progenitor cells within these axes produces cells with specific identities. The generation of different neural subtypes in the ventral neural tube depends on graded concentrations of Sonic hedgehog (Shh) along 
the d-v axis (Fig. 1.3). It was observed that the embryonic structures in rat like the notochord, the node and the floor plate express vertebrate hedgehog (vhh-1) mRNA, also known as Shh (Roelink et al., 1994). Furthermore, in vitro expression of Shh resulted in differentiation of the floor plate cells or MNs in neural plate explant cultures (Roelink et al., 1994). While Shh transfection into neural plate explants was sufficient in inducing floor plate cell and $\mathrm{MN}$ differentiation (Tanabe et al., 1995). Moreover, graded concentrations of Shh induce ventral interneuron and ventral MN differentiation (Ericson et al., 1997a; 1997b).

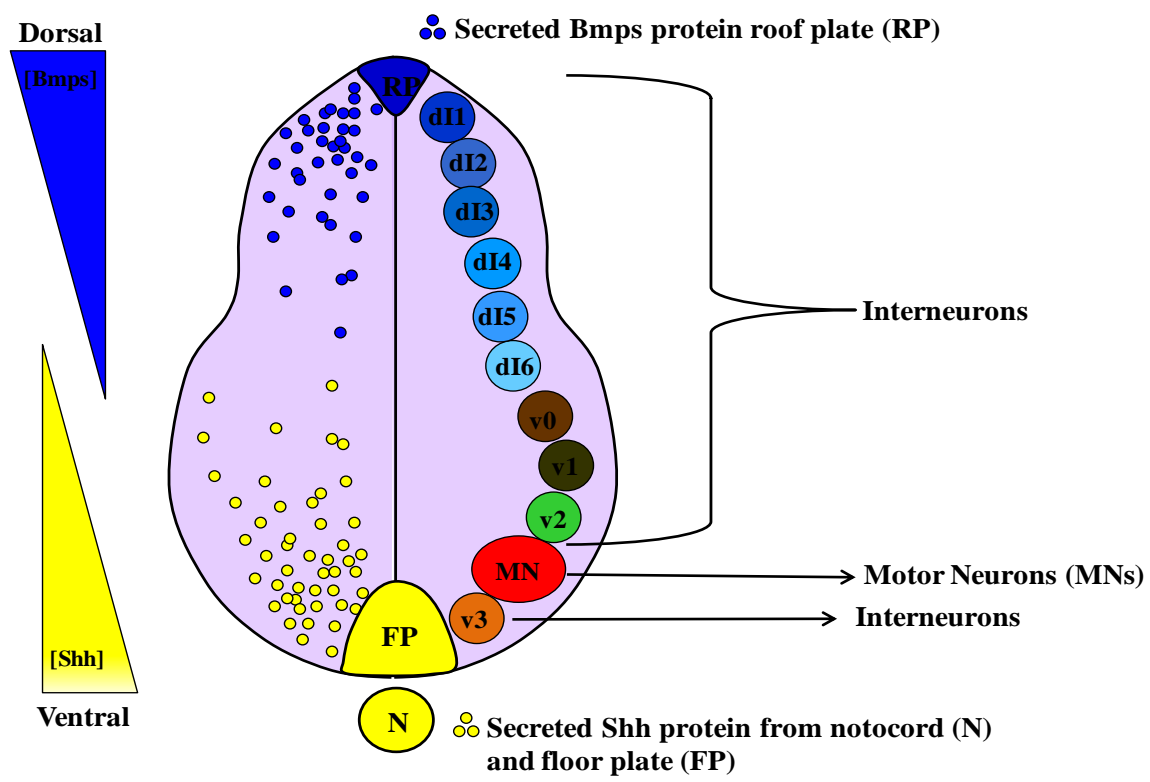

Figure 1.3 The dorsoventral (d-v) axis patterning

The neural tube's d-v axis is organized by complementary concentration gradients of secreted Bone morphogentic proteins (Bmps) and Sonic hedgehog (Shh). Roof plate (R) and surface ectodermsecreted Bmps dorsalize the neural tube, while the floor plate $(\mathrm{F})$ and notochord $(\mathrm{N})$ secreted Shh ventralizes the neural tube. Bmps generate neural progenitors to generate dorsal interneuron classes dI1-dI6, while Shh generates ventral interneuron classes (V0, V1, V2, V3) and motor neurons (MNs). (Adapted from Jessell, 2000 and Catela et al., 2015)

The graded concentrations of Sonic hedgehog (Shh) promotes the expression of certain homeodomain proteins in neural progenitor cells, which in turn form the neural progenitor domains, which are further sharpened by cross-repressive interactions between these proteins. Studies showed that Shh levels regulate two classes of homeodomain (HD) transcription factors: Shh class I proteins (Pax7, Pax6, Irx3, Dbx1, Dbx2) are repressed by Shh and class II proteins (Nkx6.1 and Nkx2.2), which are activated by Shh (Fig. 1.4A) (Briscoe et al., 2000). Class I proteins like Pax6 and Dbx2 are repressed by class II proteins like Nkx2.2 and Nkx6.1, respectively (Muhr et al., 2001). Nkx6.1 and Pax6 activate the expression of basic helix-basic-helix (bHLH) protein Olig2 expression, which promotes the 
progenitor cells to differentiate into MNs (Fig. 1.4B) (Novitch et al., 2001; Marquardt and Pfaff, 2001). More specifically, Olig2 is responsible for controlling MN subtype-specific and pan-neuronal character by activating the expression of Lhx3 and bHLH protein Neurogenin-2 (Ngn2), respectively (Novitch et al., 2001). Olig2 promotes cell cycle exit in progenitor cells directly or via Ngn2, which leads to the expression of post-mitotic markers like Is11, Is12 and $\mathrm{Hb} 9$ (Novitch et al., 2001). Moreover, the expression of $\mathrm{Hb} 9$ induces the expression of Is 1 and Lhx3, while Isl1 is required to induce Isl2 and Hb9 expression (Tanabe et al., 1998). Furthermore, $\mathrm{Hb} 9$ autoactivates its own expression to maintain differentiated MNs that express Is11, Is12, Lhx3 and ChAT, without Shh (Tanabe et al., 1998).

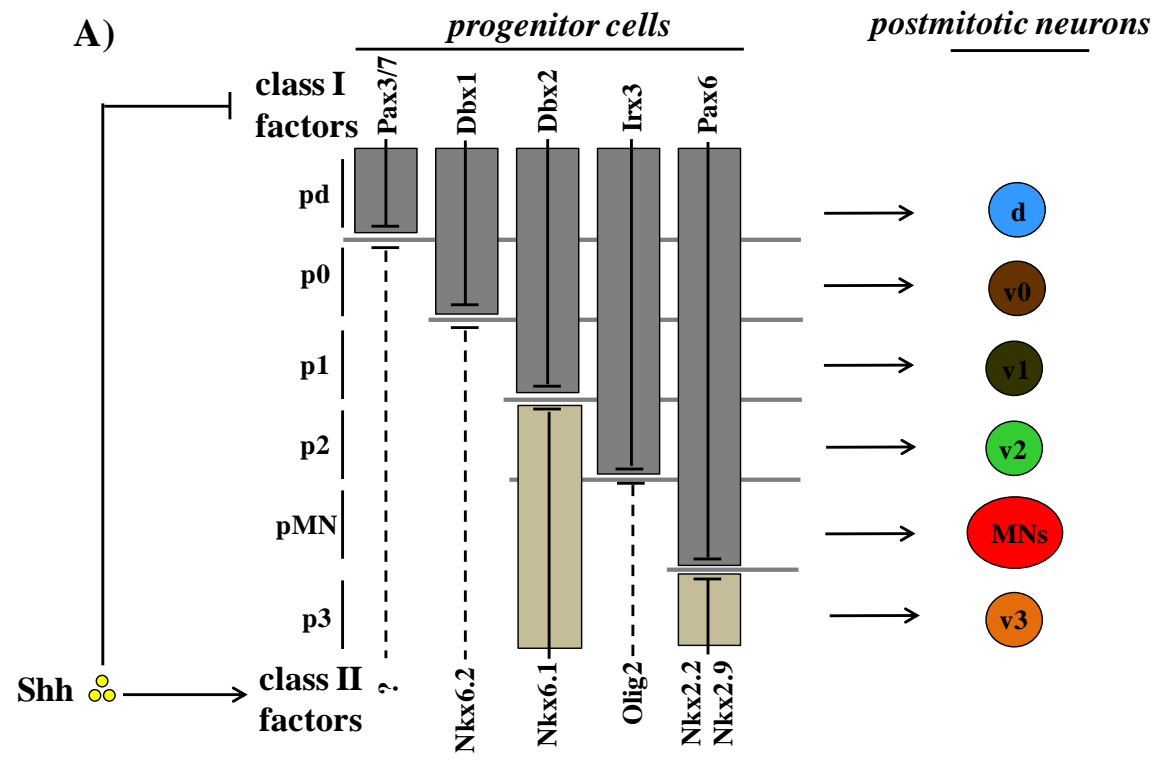

B)

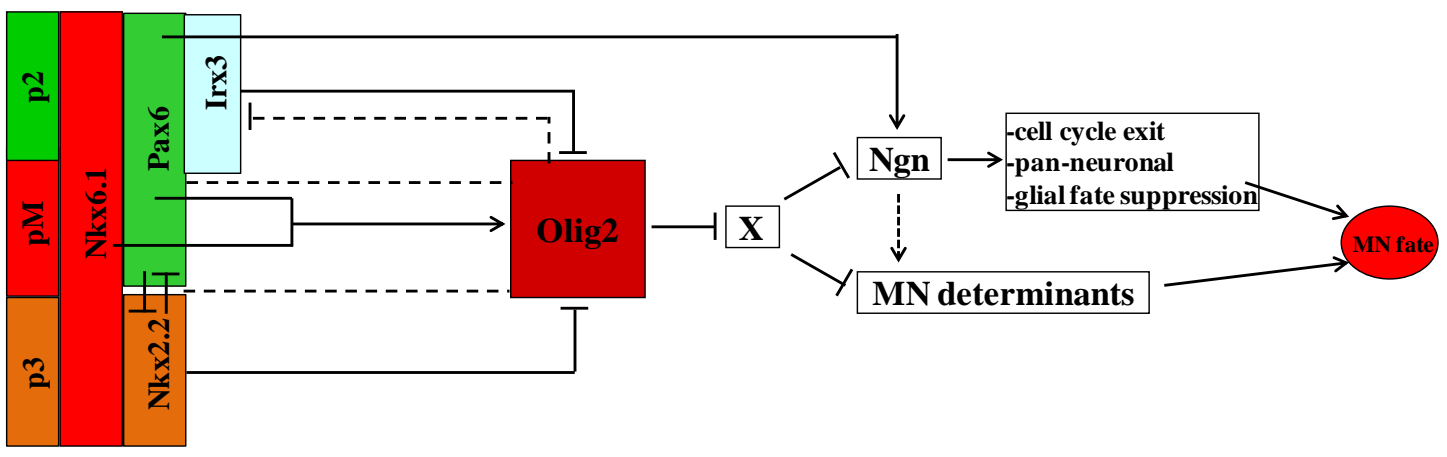

\section{Figure 1.4 Ventral neural progenitor cell identity and $\mathrm{MN}$ cell fate}

A) Neural tube Shh gradients determine whether a specific homeodomain (HD) transcription factor is expressed by a progenitor domain. The HD transcription factors expressed within a certain progenitor domain regulate neural differentiation. Shh represses class I HD transcription factors and activates class II HD transcription factors. Class I and class II HD proteins cross-repress each other resulting in specific progenitor domains. The combined expression pattern of HD proteins in the progenitor cells leads to a specific neural fate. B) The acquisition of $\mathrm{MN}$ fate is regulated by Olig2. Olig2 partially (dotted-line interaction) represses Irx3 and influences the expression of Neurogenin2 (Ngn2), through the repression of a repressor molecule X. Ngn2 influences cell cycle exit, pan-neuronal characteristics 
and suppresses glial fate, thus, driving the neurogenic lineage and the expression of $\mathrm{MN}$ determinants like HB9, MNR2, Isl1/Isl2 and Lhx3. Ngn2 is necessary but not sufficient in driving the expression of MN determinants, which is noted by dotted line. Nkx6.1 and Pax6 promote the expression of Olig2, while Nkx2.2 and Irx3 oppose the expression of Olig2. (Adapted Marquardt and Pfaff, 2001; Price and Briscoe, 2004)

\subsubsection{Refinement of MN identity based on columnar position and peripheral axonal projection}

Neurons in the neocortex and the spinal cord are organized into columnar units that form a system of recurrent and mutually connected circuits (Mountcastle, 1997; Sanders, 2002). In the spinal cord, chemical tracers were used to study axonal trajectories which revealed that $\mathrm{MN}$ somas cluster into columns representing a topological pattern correlated with the muscle groups their axons innervated (Landmesser, 1978a; 1978b; Hollyday, 1980; Gutman et al., 1993; Romanes, 1951, 1964). Thus, spinal motor "columns" containing MN somas extend across the rostrocaudal (r-c) axis of the spinal cord.

MN identities in these columns are patterned across $r-c$ axis by reciprocally graded concentrations of retinoic acid (RA) and fibroblast growth factors (FGFs), which in turn control homeobox (Hox) cluster gene expression (Bel-Vialar et al., 2002; Dasen et al., 2003; Liu et al., 2001; Catela et al., 2015) (Fig. 1.5). Hox transcription factors are encoded by 39 genes that are clustered into four complexes (HoxA, HoxB, HoxC and HoxD) that establish segmental identity and body plan patterns across the animal kingdom (Duboule and Dolle, 1989; Gaunt, 1988; Graham et al., 1989; see Philippidou and Dasen, 2013; McGinnis and Krumlauf, 1992 for review). Graded FGF concentrations determine Hox gene paralogue expression patterns at the cervical/brachial (Hox4-Hox8), thoracic (Hox8-Hox9) and lumbar (Hox10-Hox13) regions of the spinal cord (Bel-Vialar et al., 2002; Dasen et al., 2003; Liu et al., 2001; Dasen and Jessell, 2009; Philippidou and Dasen, 2013). Furthermore, RA regulates Hox gene expression at the brachial section of the spinal cord and the hindbrain (Liu et al., 2001; Glover et al., 2006; Trainor and Krumlauf, 2000; Dasen and Jessell, 2009). 


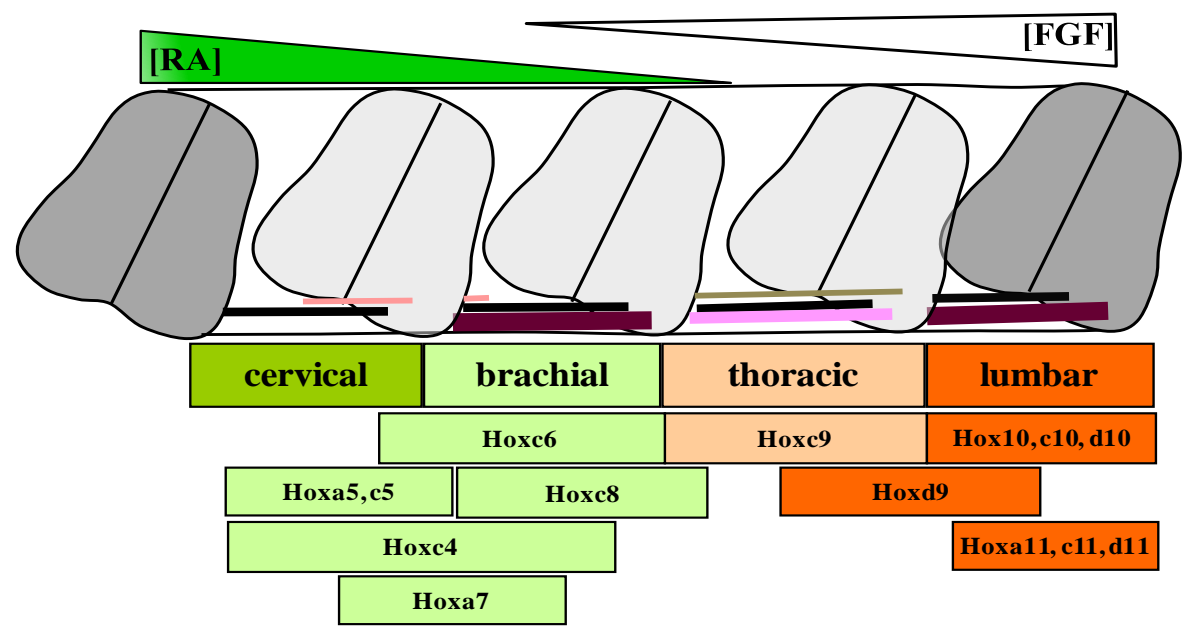

Figure 1.5 Schematic representation of the MN columns within the rostrocaudal (r-c) axis

The secretion of retionic acid (RA) and fibroblast growth factor (FGF) from opposite poles of the rostrocaudal axis ( $\mathrm{r}-\mathrm{c})$ sets up concentration gradients that induce the expression of Hox4-Hoxl1 genes. Molecularly distinct regions of the spinal cord into cervical, brachial, thoracic and lumbar and the organization of discrete motor neuron $(\mathrm{MN})$ columns is driven by the cross-repressive interactions of Hox proteins. The motor columns are organized in the r-c axis of the spinal cord: 1) cervical: the medial motor column (MMC) (black) and the phrenic motor column (PMC) (peach), 2) brachial: the medial motor columns (MMC) (black) and the lateral motor columns (LMC) (maroon), the phrenic motor column (PMC) (peach), 3) thoracic: the medial motor column (MMC) (black), the hypaxial motor column (HMC) (pink) and the preganglionic motor column (PGC) (beige), 4) lumbar: the medial motor column (MMC) (black) and the lateral motor column (LMC) (maroon). (Modified after Catela et al., 2015 and Philippidou and Dasen, 2013)

Somatic MNs send axons from these motor columns in three major routes: 1) dorsally to target axial muscles, 2) ventrally to target body wall muscles, 3) ventrally to target limb muscles (Price and Briscoe, 2004). Moreover, MNs from the preganglionic motor column (PGC) project their axons towards the sympathetic chain ganglia (scg) (Stifani, 2014). Several studies in mice have confirmed that Hox genes influence motor column identities and their precise innervations of target muscle groups (Fig. 1.6) (Philippidou et al., 2012; Lacombe et al., 2013; Wu et al., 2008; Jung et al., 2010). However, not all columnar identities are influenced by Hox genes. For instance, the medial motor column (MMC) extends throughout the spinal cord and motor axons from this column innervates midline muscles that present throughout all axial levels (Kanning et al., 2010; Philippidou and Dasen, 2013; Stifani, 2014). 


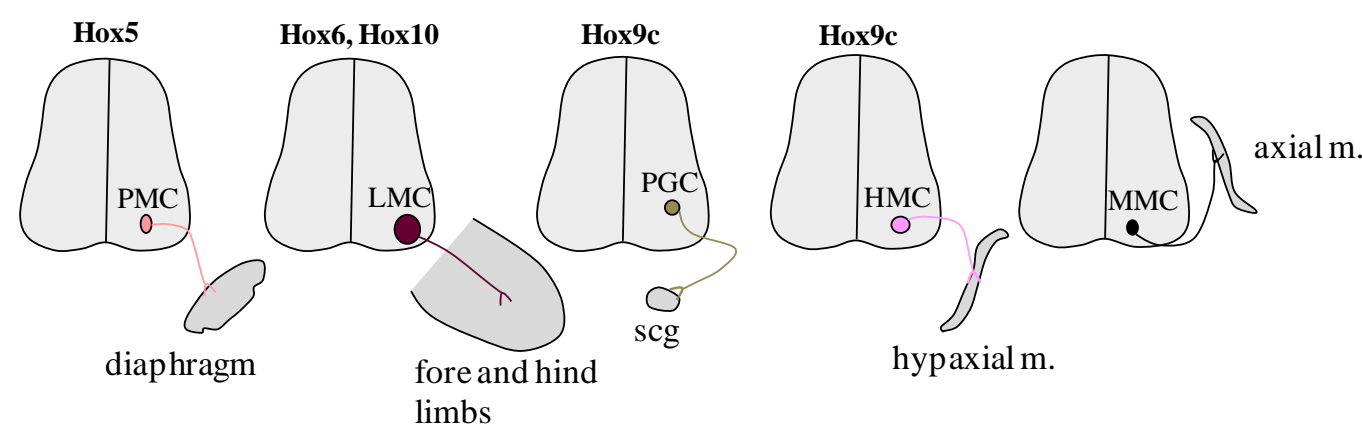

Figure 1.6 Hox genes regulate motor column specific innervations

MN identity is established by specific Hox genes: 1) Hox5 protein directs motor axons of phrenic motor column (PMC) innervation of diaphragm, 2) Hox6 and Hox10 proteins regulates motor axons of motor columns that target fore and hindlimbs, 3) Hox9c proteins directs preganglionic motor column (PGC) innervation of scg, 4) Hox9c proteins specifies the innervation of hypaxial muscles such body wall and intercostal muscles by hypaxial motor column (HMC) motor axons, 5) MMC motor column is not regulated by Hox genes but the motor axons project to axial musculature. (Modified after Catela et al., 2015 and Philippidou and Dasen, 2013)

\subsubsection{Refinement of MN subtype identity based on motor "pool" position}

MN somas cluster or form "motor pools" within the spinal columns based on the innervation of a specific muscle group (Stifani, 2014). Several studies have shown that MNs within motor pools occupy characteristic positions within motor columns, send axons to the same muscle, receive the same sensory neuron input and have a common function (Romanes, 1941; 1964; Landmesser, 2001; Hollyday, 1980; Price and Briscoe, 2004). These features imply a topographic organization of motor pools (Stifani, 2014). In addition to the motor column identities along the r-c axis, Hox gene programs also determine motor pool identities within the columns. Furthermore, studies have shown that neurotrophic factors from the mesoderm and muscles lead to the expression of ETS (E26 transformation-specific) transcription factors such as Pea3 and Er81, which are integral to precise circuit formation between MNs and sensory neurons (Haase et al., 2002; Lin et al., 1998; Dasen and Jessell, 2009). A study by Lin et al., (1998), determined the expression pattern profile of Pea3 and Er81 in chick MNs. They observed that the ETS gene expression profile in MNs paralleled most sensory neurons and their expression in MNs is contingent upon signals from the limb muscles (Lin et al., 1998). Moreover, the lack of Pea3 in mutant mice resulted in abnormal arborization within target muscle and abnormal motor pool clustering in the lateral motor column (LMC) (Livet et al., 2002). 


\subsection{Proprioception and motor control}

Various basic rhythmic patterns of limb movements have evolved in organisms that permit them to perform an array of locomotive behaviors such as swimming, crawling, walking, and flying (Kandel et al., 2000). Rhythmic motor actions of the vertebrate hindlimbs are produced by the central pattern generator (CPG) neural circuits in the spinal cord, largely independent of the sensory information (see Grillner, 2006 for review). During vertebrate movements, CPG networks comprised of interneurons produce rhythmic patterns, regulate the sequence of flexor-extensor muscles attached to joints and regulate muscle action sequence of the limbs of the body (see Kiehn, 2006 for review). Thus, automatic motor actions driving basic locomotion are generated and regulated by CPGs, however, sensory input enables the animal to adjust its gait phases to the changes in its environment (Kandel et al., 2000). Active limb movements and bending of the body triggers proprioceptive receptors like the muscle spindle (MS) and the Golgi tendon organs (GTOs), which then produce proprioceptive sensory information to regulate CPG activity during locomotion (see Pearson, 2004; Kiehn, 2016 for review). This feature is important for rectifying motor errors or adjusting to the obstacles encountered in the environment during locomotion (see Grillner, 2003 for review).

Sir Charles Sherrington described proprioception as follows: "In muscular receptivity, we see the body itself acting as a stimulus to its own receptors-the proprioceptors" (see Proske and Gandevia, 2012 for review). Proprioceptors are historically defined as receptors that enable the awareness of limb location and motion, balance, force or tension and exertion (Proske and Gandevia, 2012). The basic act of approaching an object or location reliably necessitates continuous proprioceptive and visual feedback information (Proske and Gandevia, 2012). MSs and GTOs are involved in two opposed phenomena of the nervous system: unconscious reflex activity and conscious sense of limb location and motion (Proske and Gandevia, 2012). In order to regulate body posture and locomotion, MSs continuously gauge differences in muscle length and GTOs track differences in muscle tension during mechanical alterations of joints (Proske and Gandevia, 2012; see Jami, 1992 for review).

The GTO apparatus is comprised of tendons innervated by Ib sensory afferent axons connected to the muscle fibers (Jami, 1992). GTOs function by translating differences in muscle tension between the muscle and tendons to a discharge frequency in $\mathrm{Ib}$ sensory afferent axons (Houk and Hanneman, 1967; Schoultz and Swett, 1972). They continuously track the muscle tension or the force during passive muscle stretch or voluntary muscle contraction to control movement (Granit, 1970; Schoultz and Swett, 1972). Thus, GTOs have 
a role in signaling information about muscle contraction and prevent muscle injuries during increased tension (Kandel et al., 2000). However, GTOs are implicated to have less importance in proprioception than the MSs (Grigg et al., 1973; Proske and Gandevia, 2012).

\subsection{The muscle spindle (MS) apparatus}

Precise gait patterns depend on the sensory information provided by the MS apparatus. Early studies in cat by Ruffini, (1898), established that the MS is innervated by three types of nerve endings: 1) primary afferents, 2) secondary afferents, 3) motor "plates" (see Hulliger, 1984; Matthews, 1981 for review). Matthews, (1931; 1933), recorded sensory afferent discharges from cat and frog MSs that suggested that MSs were sensitive to muscle stretch and changes in muscle stretch; in addition, sensory afferents discharged when motor nerve terminals were stimulated (Hulliger, 1984; Matthews, 1981). Various studies established that $\gamma$-MNs regulate MS activity. Leksell, (1945), showed that impeding all MN axon activity by applying pressure to the nerves was unable to cause muscle tension even though the sensory afferents innervating the MS discharged (Matthews, 1981). Hunt and Küffler, (1951a; 1951b) demonstrated that $\gamma$-MNs regulate fusimotor activity since stimulation of an individual $\gamma$-MN axon regulated the MS afferent activity, however, not causing muscle tension and thus, controlled the MS only (Matthews, 1981; Hulliger, 1984). Studies by Cooper, (1961), grouped primary (Ia) and secondary (II) MS sensory afferents on their functional differences such as the axonal conduction velocities and sensitivity to the rate of muscle stretch: primary Ia sensory afferents have higher conduction velocity and are more sensitive muscle-stretch (Cooper, 1961; Matthews, 1981; Hulliger, 1984). While studies by Matthews, (1962) established that MSs contain two functionally different types of fusimotor axons: dynamic $\gamma$-MNs $\left(\gamma_{\mathrm{d}}\right)$ and static $\gamma$-MNs $\left(\gamma_{\mathrm{s}}\right)$. Matthews, (1962) showed that the stimulation of static $\gamma-\mathrm{MN}$ axon did not result in an increase in the discharge rate of primary afferents to ramp-stretch of the soleus muscle in rat, while dynamic $\gamma$-MN stimulation increased the discharge rate of primary afferents. Furthermore, various studies lead to the morphological and physiological identification of beta motor neurons ( $\beta$-MNs) and their static and dynamic subtypes (Adal and Barker, 1965; Barker et al., 1977; Barker, 1974; Laporte et al., 1981).

MSs are innervated by different sensory afferents types, as well as $\gamma$ - and $\beta-\mathrm{MN}$ subtypes. Hence, the MSs have three different intrafusal fiber types that have unique innervations (Fig. 1.7B) (see Manuel and Zytnicki, 2011; Prochazka, 2011 for review). 


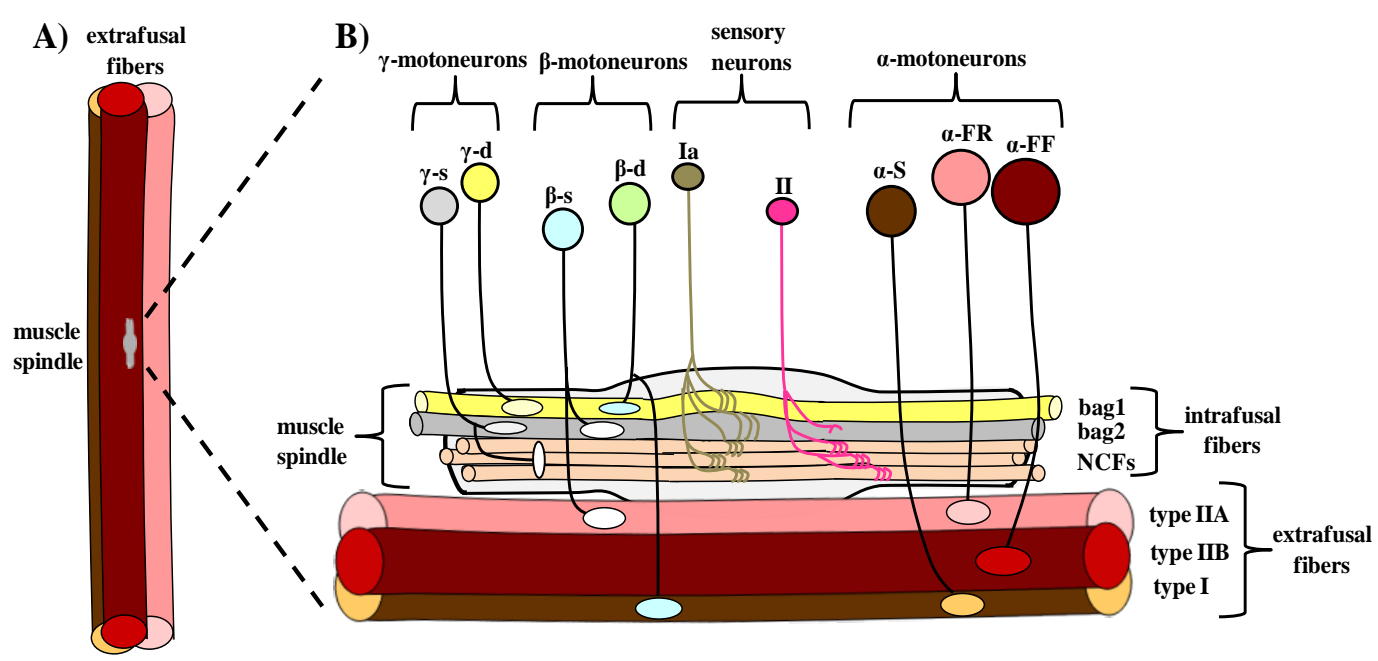

\section{Figure 1.7 Schematic depicting different types of MNs and their innervations}

A) The muscle spindle (MS) is embedded in the muscle's belly. B) $\gamma$-MNs: static and dynamic ( $\gamma$-s and $\gamma$-d) innervate intrafusal fibers bag2 and nuclear chain fibers (NCFs) or bag1, respectively. Sensory neuron group Ia and group II afferents innervate intrafusal fibers bag1, bag2, NCF or bag2, $\mathrm{NCF}$, respectively. $\beta$-MNs: static and dynamic ( $\beta$-s and $\beta$-d) innervate bag2 and type IIA or bag1 and type I fibers, respectively. $\alpha$-MNs: $(\alpha-\mathrm{S}, \alpha$-FR, $\alpha$-FF) innervate extrafusal fibers (type I, IIA, IIB), respectively. Colors: $\gamma$-MNs: $\gamma$-s (static, gray) and $\gamma$-d (dynamic, yellow); $\beta$-MNs: ( $\beta$-s, turquoise) and ( $\beta$-d, green); sensory neurons: group Ia (beige) and II (magenta); $\alpha$-MNs: $\alpha-\mathrm{S}$ (brown), $\alpha$-FR (pink), $\alpha$-FF (red); intrafusal fibers: bag1 (yellow), bag2 (grey) and nuclear chain fibers (NCFs, peach); extrafusal fibers: type I (brown), IIA (pink), IIB (red). (modified after Prochazka, 2011 and Manuel and Zytnicki, 2011)

\subsubsection{MS morphogenesis}

During embryonic development, body skeletal muscle is produced from the somites, which are structures that are generated from the paraxial mesoderm (see Chal and Porqouié, 2017 for review). There are two phases of myogenesis: primary and secondary myogenesis. Primary myogenesis consists of progenitor cells forming primary myocytes that generate the early limb muscles and myotomes (Chal and Porqouié, 2017). While secondary myogenesis consists of a group of progenitors that fuse with the same progenitors or with primary myofibers to generate secondary fibers, which maintain muscle growth (Chal and Porqouié, 2017). Positioned parallel to the skeletal muscle fibers are the MSs, which differentiate embryonically and mature postnatally. Studies have shown that sensory neuron denervation during the late embryonic and early postnatal stages results in deteriorated intrafusal fibers and the lack of MS differentiation (Zelená, 1957; Zelená, 1976). Furthermore, MSs in rat soleus, gastrocnemius and extensor digitorum longus muscles differentiated normally despite lacking the fusimotor axon innervations (Zelená and Soukup, 1973). Kucera and Walro, (1992) confirmed that MS development requires sensory afferent innervation but not $\gamma-\mathrm{MN}$ 
innervation. A molecular mechanism for MS differentiation was revealed through several studies. Ernfors et al., (1994) observed that neurotrophin-3 $\left(N T-3^{-/}\right)$mutant mice at birth lacked muscle spindle (MS) and Golgi tendon organ (GTO) structures in the soleus muscle, and showed a decreased number of Ia sensory neurons. Furthermore, the lack of NT-3 receptor, tropomyosin receptor kinase $\mathrm{C}$ (TrkC) in $\operatorname{TrkC}^{-/}$mutant mice resulted in the lack of Ia sensory afferent projections to $\mathrm{MNs}$ and the mice displayed defects in posture and movements (Klein et al., 1994). Hippenmeyer et al., (2002), demonstrated that Neuregulin (Nrg-1) isforms (Ig-Nrg1), expressed by sensory neurons located in the dorsal root ganglion (DRG) signaled the expression of Pea3, Egr3, and Erm in the MS of E15.5 animals through its ErbB receptors, while the lack of Ig-Nrg1 isoforms in mice resulted in immature sensory afferent annulospiral endings and abnormal MS differentiation (Hippenmeyer et al., 2002). Studies have shown that intrafusal fibers express transcription factors like Egr3, Pea3, Er81, Erm that are important for MS development (Tourtellotte and Milbrandt, 1998; Arber et al., 2000; Hippenmeyer et al., 2002). Tourtellotte and Milbrandt, (1998) generated $\mathrm{Egr}^{-/-}$mutant mice (growth response-3 (Egr-3) zinc-finger transcription factor) and observed that these mice lacked MSs and showed ataxic gait, thus, Egr3 regulates MS morphogenesis (Tourtellotte and Milbrandt, 1998). Moreover, Arber et al., (2000) observed that Er81 mutant mice had normal MS differentiation at E18, however, these mutants showed abnormal intrafusal fiber differentiation after birth.

The differentiation of MS in mice is initiated at E14.5 (embryonic day 14.5) by the binding of Nrg-1 secreted by Ia sensory afferents to ErbB receptors on intrafusal fibers that in turn leads to the expression of transcription factors like Egr3, Pea3, Erm and other factors and eventual branching of Ia sensory afferents (Hippenmeyer et al., 2002). Furthermore, during the early postnatal (P0-P7), intrafusal fibers express factors like NT-3 and Er81 that play a role in Ia sensory afferent innervation MNs and thus, maintenance of the MS (Hippenmeyer et al., 2002). Studies in rat have shown that $\gamma$-MN innervation of MS commences by birth while these innervations are not mature until two-weeks postnatally (Milburn, 1973a; 1973b). Furthermore, MSs in mice become morphologically mature during the third week of postnatal development (P20) (Sonner et al., 2017).

\subsubsection{The role of $\gamma$-MNs in the physiological function of the MS}

The MS is special amongst sensory receptors since its sensitivity can be regulated by the CNS through $\gamma$-MNs (Fitz-Ritson, 1982; Hulliger, 1984). One function of $\gamma$-MNs is to allow the sensory afferents to signal the changes in muscle length continuously during large 
muscle contractions (Kandel et al., 2000). Therefore, $\gamma$-MNs allow for fluid muscular action by enabling direct control of muscle spindle sensitivity, which otherwise would be active during the stretch and movement velocity only (Taylor, 1972; see Murthy, 1978 for review). The loss of $\gamma$-MN function due to disease has effects on muscle tone, reflexes and voluntary movements (Murthy, 1978). For instance, abnormal function of $\gamma$-MNs in multiple sclerosis (MS) leads to spasticity and abnormal $\gamma$-MN drive in Parkinson's disease (PD) leads to rigidity in muscles (Dietrichson, 1971; Szumski et al., 1974; Hagbarth et al., 1975; Murthy, 1978). Thus, $\gamma$-MNs are integral to the function of the MS receptor and thus, proprioceptive movement control.

Studies have shown that proprioceptive circuit "feedback" regulates swing and stance phase changes during locomotion in cat (Grillner and Rossignol, 1978; Duysens and Pearson, 1980; Hiebert et al., 1996; Lam and Pearson, 2001; Mcvea et al., 2005). Moreover, studies revealed that Egr3 mutant mice displayed longer activity in the ankle flexor tibialis anterior (TA) muscle during the swing phase of stepping cycle, thus, leading to aberrant foot trajectories due to lack of group Ia/group II afferent input (Akay et al., 2014). Furthermore, proprioceptive information from MSs and GTOs organizes movements of the hip and knee, while hip and ankle joint movements depend solely on MSs (Akay et al., 2014). Hence, proprioceptive feedback information from MSs and GTOs is essential for normal walking patterns in mice (Akay et al., 2014).

\subsubsection{1 $\alpha-\gamma$ co-activation without $\gamma$-MN input}

Muscle contractions lead the MS intrafusal fibers to relax or "slack" and cause the sensory afferents that innervate them to become insensitive to various muscle lengths (Kandel et al., 2000). However, this is normally prevented since the $\gamma-\mathrm{MNs}$ activate almost simultaneously with $\alpha$-MNs, thus, keeping the intrafusal fibers stretched or "taut," which in turn allows the sensory afferents to remain sensitive during shortening of the muscle (Kandel et al., 2000). This mechanism of " $\alpha-\gamma$ co-activation" occurs during voluntary movements to allow the MS to function and signal muscle length shifts and rate during various degrees of contractions in muscle (Fig. 1.8A-C) (Kandel et al., 2000). Furthermore, $\alpha-\gamma$ co-activation is utilized to control various rhythmic movements including stepping, respiratory, masticatory and scratching (Perret and Buser, 1972; Perret and Berthoz, 1973; Corda et al., 1966; Cody et al., 1975; Feldman, 1977; Murthy, 1978). In summary, the $\alpha-\gamma$ co-activation enables precise activation levels for the MSs resulting in a programmed sensitivity to report shifts in muscle length and rate during muscle contraction (Fig.1.8A-C) (Kandel et al., 2000). 
A)
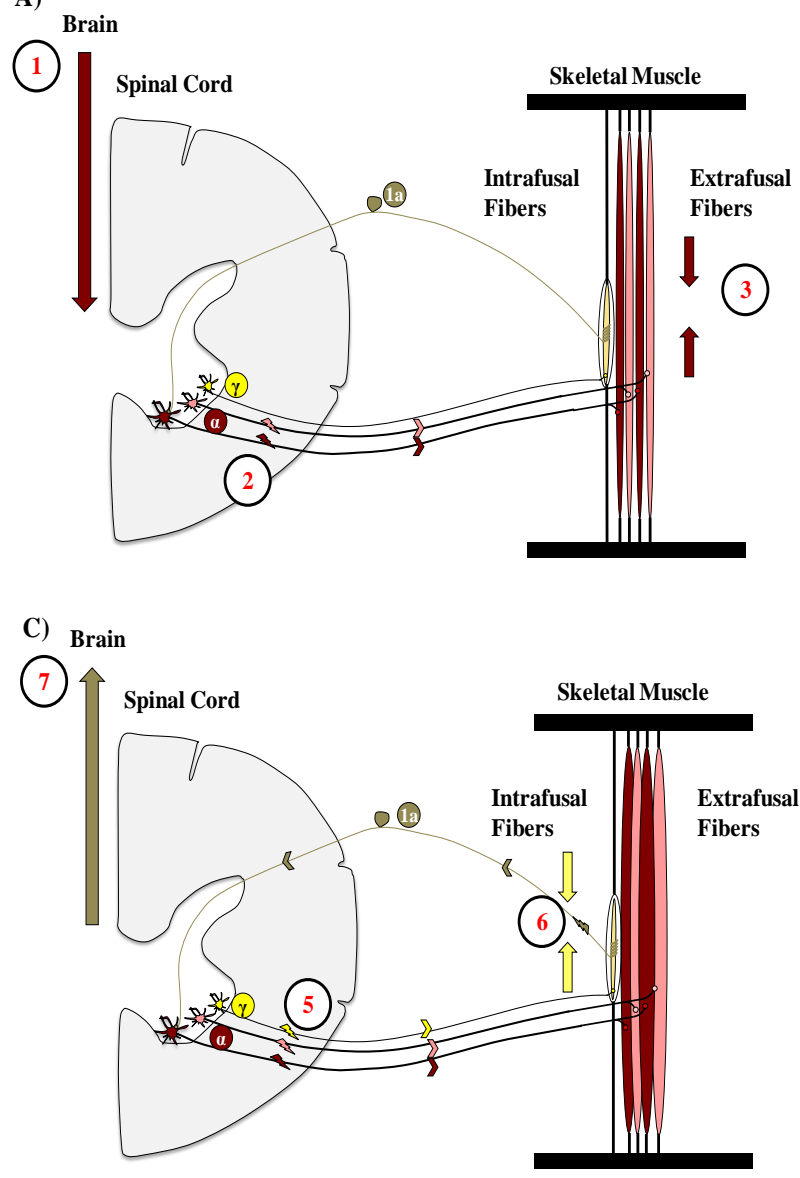

B)

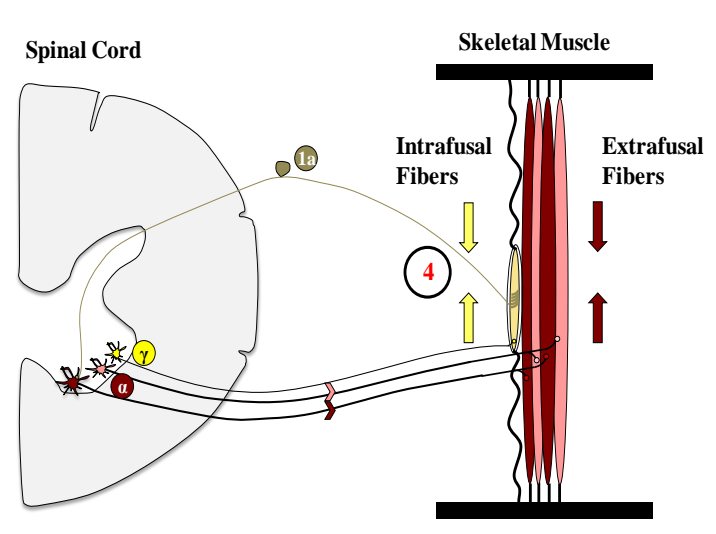

\section{Figure 1.8 Schematic representation of $\alpha-\gamma$ co-activation}

A) The muscle spindle (MS) circuit: $\gamma$-MN (yellow) efferents innervate the polar region of the MS intrafusal fibers (light yellow) and Ia sensory (beige) afferents innervate the central region of the MS. 1) The CNS sends signals to contract a muscle (for example, the soleus/calf muscle in the leg), 2) which causes $\alpha$-MNs (red and pink) to fire, 3) resulting in extrafusal fibers (red and pink) to contract. B) Simultaneously, intrafusal fibers stretch. 4) However, if a failure in $\gamma$-MN stimulation had occurred, the intrafusal fibers would remain loose or "slacken" and no information about the changes in muscle length or the rate of change in muscle length would be presented to CNS because the Ia sensory afferents would become insensitive. C) Since the fusimotor system in normal muscle is intact, 5) a simultaneous stimulation of $\gamma$-MNs at the polar ends of intrafusal fibers is seen with $\alpha$-MN fire and extrafusal fiber contraction. 6) This keeps the intrafusal fibers tightened or "taut" and Ia sensory neurons are able to 7) signal information about these shifts in muscle length to CNS, thus, keeping the MS sensitive during various ranges of muscle contraction. (Adapted from Kandel et al., 2000)

\subsubsection{The stretch reflex}

In order to adjust to changes in the environment, proprioceptive reflexes influence voluntary movements (Kandel et al., 2000). Proprioceptive reflexes regulate voluntary movements in animals through two properties: 1) "stretch reflex," and 2) closed "feedback" 
loops (Kandel et al., 2000). The property of a muscle to oppose lengthening is known as the "stretch reflex" (Fitz-Ritson, 1982). When a muscle is stretched, there is an increase in the firing of sensory afferents that synapse onto $\alpha$-MNs (Kandel et al., 2000; Fitz-Ritson, 1982). The $\alpha-M N s$ also become activated leading to a muscle contraction of the same muscle they innervate (Kandel et al., 2000; Fitz-Ritson, 1982). Following this muscle contraction, the Ia sensory afferent firing decreases causing a decrease in the muscle length which permits the cycle to repeat, a property termed the stretch reflex loop (Kandel et al., 2000). This stretch reflex loop is in a constant cycle to maintain the muscle length at precise magnitudes, thus, the difference in muscle length are used as information to repeat the cycle (Kandel et al., 2000). This stretch mechanism can also be thought of as a negative feedback system or "servo" control system that governs muscle length (Kandel et al., 2000; see Taylor 1970 for review). The CNS input onto $\alpha$ - and $\gamma$-MNs regulates the stretch reflex mechanism, which enables the fine-tuning of voluntary movements and body posture changes (Hunt and Ottoson, 1976; Fitz-Ritson, 1982).

The $\gamma$-MNs regulate sensory afferent and intrafusal fiber systems for dynamic and static tasks (Fitz-Ritson, 1982). For instance, $\gamma_{\mathrm{d}}$-MNs stimulate dynamic bag1 fibers that result in a higher receptivity of the sensory afferent axons and higher activation of $\alpha$-MNs to variations in muscle length, thus, generating a more robust stretch reflex mechanism to control muscle length during locomotion (Fitz-Ritson, 1982). When $\gamma_{\mathrm{s}}-\mathrm{MNs}$ stimulate static bag2 fibers, the highly stimulated MS activates muscles with a higher force for all muscle length variations, without changing stretch reflex properties, which can be utilized for regulating posture (Fitz-Ritson, 1982).

\subsubsection{Beta motor neurons ( $\beta$-MNs)}

Various studies have observed that $\beta$-MNs in both mammals and lower vertebrates like frogs, comprise of one-third of all muscle units and innervate three-fourths of all the MSs in mammals (see Hulliger, 1984; Manuel and Zytnicki, 2011 for review). Despite their abundance, their functional role has not been studied extensively, although they are thought to potentially regulate movement and posture, but their functions in mammals may rather be "assistive" to $\gamma$-MNs (Manuel and Zytnicki, 2011).

\subsection{MN functional diversification}

Motor pools are suggested to be arranged topographically according to their peripheral muscle targets: rostral motor pools form circuits with proximal muscles of the 
limb, while lateral motor pools connect to dorsal muscles of the limb and medial motor pools connect to ventral muscle groups of the limb (Landmesser 1978a; Romanes 1951; Vanderhorst and Holstege, 1997; see Kanning et al., 2010 for review). In humans, there are in excess of 120,000 MNs that innervate more than 100 million muscle fibers within approximately 300 pairs of muscles of the body (Kanning et al., 2010). The mammalian spinal cord contains well identified classes of MNs, $\alpha$ - and $\gamma$-MNs, while a third class, $\beta$ MNs, are less well characterized (Burke, 2004; Kanning et al., 2010). The $\alpha$ - and $\beta$-MNs comprise two-thirds of the total number of MNs, while $\gamma$-MNs account for one-third of the total MNs (Burke et al., 1977; Friese et al., 2009). Within a motor pool, several common characteristics of MNs can categorize them into functional subtypes: their morphology, their peripheral and central synaptic connectivity, molecular marker expression and biophysical properties (Kanning et al., 2010). A summary of these MN properties is provided in Fig. 1.9A-B.

\subsection{1 $\alpha$ - and $\gamma$-MN Ia afferent connectivity and morphology}

MN connectivity and biophysical properties ultimately contribute to how they control motor behavior. $\alpha$ - and $\gamma$-MNs differ in their sensory neuron connectivity. While $\alpha$-MNs receive monosynaptic Ia input from proprioceptive sensory neurons, $\gamma$-MNs lack this direct input (Burke et al., 1977; Westbury, 1982; Friese et al., 2009; Shneider et al., 2009). $\alpha$-MNs on average have a soma diameter two times the size of $\gamma$-MNs, on average outnumber $\gamma$-MNs by three times within motor pools and have more intricate dendritic arborizations than $\gamma$-MNs (Burke et al., 1977; Westbury, 1982; Friese et al., 2009; Shneider et al., 2009).

\subsubsection{Molecular correlates distinguishing $\alpha-$ and $\gamma-\mathrm{MNs}$}

Neurons can be classified based on their morphology, connectivity, physiology and gene expression patterns (Sanes and Masland, 2015). The majority of neurons in the nervous system express RNA-binding protein, fox-1 homolog 3 (Rbfox3, NeuN) that distinguishes them from non-neuronal cell types (Mullen et al., 1992). MNs are cholinergic neurons and therefore express choline acetyltransferase (ChAT), an enzyme that catalyzes acetylcholine synthesis, in addition to expressing solute carrier family 18 member A3 (Slc183a, vAChT), which packages acetylcholine into synaptic vesicles (Wu and Hersh, 1994; Nagao et al., 1998; Tanabe et al., 1998; Friese et al., 2009).

$\alpha$-MNs and $\gamma$-MNs can be distinguished molecularly during the late stages of embryonic spinal cord development by their expression of wingless-type MMTV integration 
site family, member 7A (Wnt7a) at E17.5 in the mouse spinal cord (Ashrafi et al., 2012). In the postnatal spinal cord, $\alpha-\mathrm{MNs}$ express high levels of the RNA-binding protein, fox-1 homolog 3 (Rbfox3, NeuN) and low to negligible levels of estrogen-related receptor gamma (Esrrg, Err3) (Friese et al., 2009). In contrast, $\gamma$-MNs do not express NeuN, but express high levels of Err3 (Friese et al., 2009). Other postnatal $\gamma$-MN markers that have been recently identified are 5-hydroxytryptamine (serotonin) receptor 1D (Htr1d) and GDNF family receptor alpha 1 (Gfr $\alpha-1)$, as well as ATPase, $\mathrm{Na}^{+} / \mathrm{K}^{+}$transporting, alpha 3 polypeptide (Atp1a3) (Enjin et al., 2010; Shneider et al., 2009; Edwards et al., 2013).

\subsubsection{Molecular differences among $\alpha$-MN subtypes: fast $\alpha$-MNs and slow $\alpha$-MNs}

Neuronal input from the brain and the sensory system is translated into force to produce movement through motor units. The motor unit is comprised of a $\mathrm{MN}$ and the muscle fibers innervated by its axon (Heckman and Enoka, 2012). The muscle unit consisting of MN axon and muscle fibers it innervates are shown to have mechanical properties that can classify the motor unit types (Burke et al., 1973). The different mechanical properties of the muscle units enable the classification of the motor unit types: 1) twitch time to peak tension, 2) the "sag" of an unfused tetanic contraction, 3) the fatigability of the muscle unit to repetitive tetanic activation (Zengel et al., 1985). Thus, $\alpha-\mathrm{MN}$ motor units can be classified into subtypes based on the contractile properties of the muscle units. For example, there are three major types of motor units for $\alpha$-MNs: 1) $\alpha$-FF (fast-twitch, fatigable), 2) $\alpha$-FR (fasttwitch fatigue-resistant), 3) $\alpha-S$ (slow-twitch, fatigue-resistant) (Kanning et al., 2010). Furthermore, fast $\alpha-\mathrm{MNs}$ and slow $\alpha$-MNs express distinct molecular correlates. Müller et al., (2014) identified Delta-like 1 homolog (Dlk1) to be expressed by fast $\alpha$-MNs, which is necessary and sufficient for producing fast $\alpha$-MN biophysical properties. The forced expression of Dlk1 in late-gestation chick neural tube followed by whole cell patch-clamp recordings in MNs showed shifted biophysical properties towards higher firing threshold and firing frequencies and lower firing durations and after-hyperpolarization (AHP) (Müller et al., 2014). Furthermore, MNs recorded in $D l k l^{-/-}$mutant mice showed biophysical properties resembling slow/intermediate $\alpha$-MN types (Müller et al., 2014). Recently, Kaplan et al., (2014), demonstrated that matrix metallopeptidase 9 (Mmp9) is expressed by fast $\alpha$-MNs that are prone to degenerate in mouse model of human Amyotrophic Lateral Sclerosis (ALS) (SOD1 ${ }^{\mathrm{G} 93 \mathrm{~A}}$ mice). Other $\alpha-\mathrm{MN}$ subtype markers are chondrolectin (Chodl) and calcitonin/calcitonin-related polypeptide, alpha (Calca), which are co-expressed by large soma MNs, whereas estrogen-related receptor, beta (Esrrb, Err2) was found to be expressed 
by relatively small soma size and non-cholinergic cells (Enjin et al., 2010). The authors suggested that Chodl could be expressed by fast $\alpha$-MNs since $\mathrm{Chodl}^{+}$cells recorded using whole cell patch-clamp technique showed short afterhyperpolarization (AHP) half-decay time and high rheobase current (Enjin et al., 2010). Furthermore, the authors proposed that Err2 is a molecular correlate of slow $\alpha-\mathrm{MNs}$ since RNA in situ hybridization and immunodetection studies in P11 $\mathrm{Egr3}^{-/-}$mutant mice with degenerated MSs leading to lack of $\gamma$-MNs seemed to show no changes in Err2 expression pattern (Enjin et al., 2010). Studies have shown that synaptic vesicle glycoprotein 2 a (Sv2a) is expressed in motor terminals that innervate type I and small type IIA muscle fibers and thus, is a molecular correlate of slow $\alpha$-MNs (Chakkalakal et al., 2010).

\subsubsection{MN biophysical properties}

MNs have complex biophysical properties that determine their functional role in controlling motor behavior. MN properties depend on two interlinked characteristics of the cell membrane: 1) MN morphology consisting of MN size (cell membrane area) and $\mathrm{MN}$ structural organization (cell membrane geometry), or 2) $\mathrm{MN}$ intrinsic properties (cell membrane ion channels) (Burke and Rudomin, 2011; Burke, 2011).

MN properties directly related to $\mathrm{MN}$ morphology are 1) axonal conduction velocity (CV), 2) input resistance $\left(\mathrm{R}_{\mathrm{N}}\right), 3$ ) dendritic electronic length (L) (Burke, 2011). Specifically, the input resistance $\left(\mathrm{R}_{\mathrm{N}}\right)$ is the resistance of an $\mathrm{MN}$ to the input of current, thus a property that shows whether the MN depolarizes due to current (Kandel, 2000; Burke, 2011). The MN properties that are intrinsic properties of the cell membrane are 1) the membrane time constant $\left.\left(t_{m}\right), 2\right)$ the after-hyperpolarization (AHP), and 3) the voltage-dependent biophysical properties (Burke and Rudomin, 2011; see Burke, 2011 for review). These properties are influenced by ion channel diversity in MNs as well as their morphology (Heckman and Enoka, 2004).

MNs within a motor pool can have a ten-fold difference in rheobase current $\left(I_{r h}\right)$, a property representing the membrane excitability, which is defined as the current required to fire an action potential (Carp, 1992; Fleshman et al., 1981; Gustaffson and Pinter, 1984; Pinter et al., 1983; Power and Binder; 1985; see Binder et al., 2011 for review). The rheobase current depends upon the input resistance and the voltage-gated ion channels that may be active prior to the MN reaching its threshold potential (Enjin et al., 2012). In general, smaller MNs have a lower rheobase compared to larger MNs. The voltage-threshold $\left(\mathrm{V}_{\text {thr }}\right)$ depends on $\mathrm{Na}^{+}$ion channel sensitivity, thus, controlling the voltage at which the MN will fire an 
action potential (Heckman and Enoka, 2004). In general, smaller MNs have a lower $\mathrm{V}_{\text {thr }}$ than larger MNs.

Similar to other nervous system neurons, the repetitive $\mathrm{MN}$ action potential is comprised of initial inwardly directed $\mathrm{Na}^{+}$ion conductance and followed by outwardly directed $\mathrm{K}^{+}$ion conductance (Araki et al., 1962; Burke and Rudomin, 2011). The membrane potential $\left(\mathrm{V}_{\mathrm{m}}\right)$ repolarizes due to the effects of $\mathrm{K}^{+}$ion conductances activating with a delay, the inactivation of $\mathrm{Na}^{+}$ion conductances, and the effects of leak channel conductances (Binder et al., 2011). More specifically, the fast repolarization phase seen in action potentials is responsible for fast activating voltage-sensitive $\mathrm{K}^{+}$ion channel conductance, while the slow activating voltage-sensitive $\mathrm{K}^{+}$ion channel conductance generates the afterhyperpolarization (AHP) phase after the action potential, which is mainly due to $\mathrm{Ca}^{2+}$ mediated $\mathrm{K}^{+}$conductances $\left(\mathrm{g}_{\mathrm{KCa}}\right.$ ) (Schwindt and Crill, 1981; Barret et al., 1980; Rudy, 1988; Heckman and Enoka, 2004). $\mathrm{Ca}^{2+}$ ion influx into the MN during the action potential activates the $\mathrm{g}_{\mathrm{KCa}}$ conductances, while intracellular $\mathrm{Ca}^{2+}$ diffusion, pumping and binding intracellular proteins determines the AHP decay times, thus, making AHP a critical property in determining MN firing rate (Kernell, 1965b; Heckman and Enoka, 2004; Binder et al., 2011).

MNs fire repetitive action potentials when the depolarizing current incorporates beyond their $\mathrm{V}_{\text {thr }}$, a property that depends on the time and quantity of current (Heckman and Enoka, 2004; Binder et al., 2011). The firing frequency correlates with the quantity of injected current, which is termed rate-modulation (frequency-current $F$-I, curve) and the slope of this relationship is defined as the gain (Kernell, 1965a; Schwindt and Calvin, 1972; Schwindt, 1973; Heckman and Enoka, 2004). Studies have shown that the F-I curve observed during rhythmic firing in cat lumbar MNs have a primary-range (shallow F-I curve slope) and secondary-range (steep F-I curve slope), a characteristic observed in other CNS neurons and hypothesized to be dependent upon balancing of influx and efflux conductances (Schwindt and Crill, 1982; Kernell, 1965a; Grantyn and Grantyn, 1978). The discharge rate or firing frequency decreases over time, which is called the spike-frequency adaptation (Binder et al., 2011).

\subsubsection{Biophysical properties of $\alpha-\mathrm{MN}$ innervating specific motor units in cat and rat}

There is a functional link between $\alpha-\mathrm{MN}$ morphology and electrical properties and the amount motor unit maximum force, isometric twitch speed and endurance (Kanning et al., 2010). Building on the work of Eccles, (1955), Eccles et al., (1957a; 1957b), Burke et al., (1973), studies performed by Zengel et al., (1985) provided insight into how different MN 
biophysical properties correlate with their muscle units, thus allowing the classification of $\alpha$ MN motor unit type. Zengel et al., (1985) used intracellular recordings to characterize the biophysical properties of cat medial gastrocnemius MNs corresponding to the muscle unit controlled by these MNs. They determined that the MN rheobase pattern was correlated with the motor units: fast-twitch, fatigable $(\alpha-\mathrm{FF})>$ fast-twitch, fatigue-resistant $(\alpha-\mathrm{FR})>$ slowtwitch, fatigue-resistant $(\alpha-S)$. The input resistance showed the opposite pattern of $\alpha$-FF $<\alpha-$ FR $<\alpha-S$ (Zengel et al., 1985). They observed that AHP half-decay time and the AHP duration had a pattern of $\alpha-\mathrm{FF}, \alpha-\mathrm{FR}<\alpha$-S (Zengel et al., 1985). Overall, their study showed that MN membrane properties of rheobase, input resistance and AHP half-decay time and duration classified the motor unit types with high accuracy (Zengel et al., 1985). Functionally, MN membrane properties may determine the muscle activity by directing the sequential recruitment of motor units from $\alpha-S$ to $\alpha-F R$ to $\alpha-F F$ for normal movements (Zengel et al., 1985). Furthermore, studies in rats described a similar relationship between the MN biophysical properties and the motor unit type (Gardiner, 1993). To summarize, studies have suggested that neuromuscular system seems to be organized for the control of movement according to Henneman's size principle, which links MN anatomy and biophysical properties with muscle unit size, ultimately, ensuring sequential activation of motor unit from smallest to largest (Henneman et al., 1965a; 1965b; Gordon et al., 2004).

\subsubsection{Characterization of $\alpha$ - and $\gamma$-MN biophysical properties in cat}

Early studies using intracellular recordings suggested that cat $\alpha$ - and $\gamma$-MNs have unique biophysical properties. Eccles et al., (1960), identified $\gamma$-MNs in the cat ventral spinal cord by antidromic volleys, which are the spike potential volleys extracellularly recorded in MNs after nerve stimulation. $\gamma$-MNs showed slower spike potential volleys than $\alpha$-MNs (50$110 \mathrm{~m} / \mathrm{sec})$ because they have a slower conduction velocity $(15-50 \mathrm{~m} / \mathrm{sec})$ due to their smaller axon diameter (Kuffler et al., 1951; Hunt and Kuffler, 1951a). Moreover, intracellular recordings on identified $\gamma$-MNs revealed that $\gamma$-MNs have a higher discharge rate than $\alpha$-MNs (Hunt, 1951; Kobayashi et al., 1952; Hunt and Paintal, 1958). Intracellular recordings of $\gamma$ MNs located in the lumbosacral region of the spinal cord in decerebrate cats showed that $\gamma$ MNs have higher discharge rates and lower thresholds for discharge than $\alpha-\mathrm{MNs}$ (Kemm and Westbury, 1978). Furthermore, they observed the membrane input resistance to be smaller in $\gamma$-MNs than in $\alpha$-MNs (Kemm and Westbury, 1978).

Recently, putative $\alpha$ - and $\gamma$-MN biophysical properties were analyzed in early postnatal mice. RNA in situ hybridization studies in the postnatal day 11 (P11) mice showed 
that relatively small soma size $\left(100-400 \mu \mathrm{m}^{2}\right)$ cells expressed serotonin receptor $1 \mathrm{~d}(5 \mathrm{ht}-1 \mathrm{~d})$ mRNA which co-localized with vAChT mRNA (Enjin et al., 2012). They performed whole cell patch-clamp recordings in GFP reporter mice (serotonin $1 \mathrm{~d}:: \mathrm{GFP}^{+}$) and characterized the biophysical properties in putative $\gamma$-MNs during early postnatal development (P0-P6) (Enjin et al., 2012). The mean input resistance for putative $\gamma$-MNs was in between the range of fastlike $\alpha$-MNs (low mean) and slow-like $\alpha$-MNs (high mean) and was significantly different between all three MN subtypes at these stages (Enjin et al., 2012). The mean rheobase current for putative $\gamma$-MNs was in between the range of fast-like $\alpha$-MNs (high mean) and the slowlike $\alpha$-MNs (low mean), which was also significantly different for all three MN subtypes (Enjin et al., 2012). Finally, the mean AHP half-decay time was also in the midrange for putative $\gamma$-MNs when compared to fast-like $\alpha$-MNs (low mean) and slow-like $\alpha$-MNs (high mean), which was also significantly different between all three $\mathrm{MN}$ subtypes (Enjin et al., 2012). However, the mean firing frequency did not differ significantly between putative $\gamma$ MNs and both $\alpha$-MNs types (Enjin et al., 2012).

Studies have shown that MSs in cat, rat and mouse develop pre- or perinatally (Milburn, 1984; Kucera et al., 1988; Banks et al., 2009; Sonner et al., 2017). However, the complete maturation of MS morphology and physiology occurs postnatally, which is important for $\gamma-\mathrm{MN}$ function as well. For instance, in the cat, the tonic discharges from the MS were observed at P6-P10, while the $\gamma$-MN discharges were observed at P17 (Skoglund, 1960). In the mouse, MS had mature morphology at the postnatal age of P20 (Sonner et al., 2017). Thus, it is possible the differences observed in $\gamma$-MN biophysical properties between cat and mouse studies were due to differences in the developmental stages at which the studies were carried out. Enjin et al., determined the biophysical properties of $\gamma-\mathrm{MN}$ at P0P6; a developmental stage at which MSs are immature. While, studies in cat by Kemm and Westbury, (1978), were carried out in young cats, which have morphologically and physiologically mature MSs. Thus, since these MS are different developmentally, the $\gamma$-MNs innervating may have different biophysical properties. 


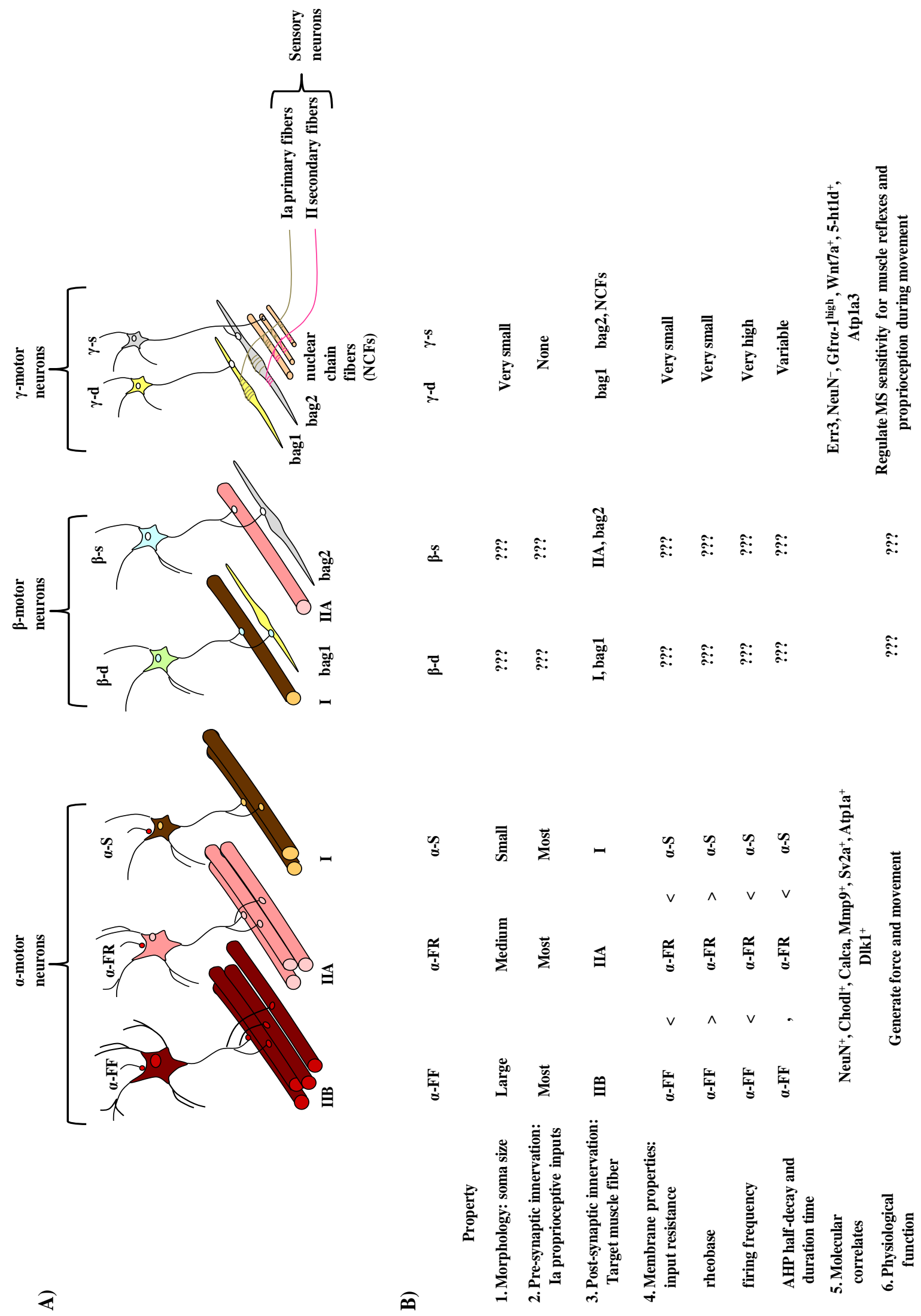

Figure 1.9 General patterns of properties in different MN subtypes

A) Studies in cat and mice have shown that MNs differ according to their morphology, pre- and postsynaptic inputs, biophysical properties, molecular correlates and physiological function. B) 1) $\alpha$-MNs 
$(\alpha$-FF, $\alpha$-FR, $\alpha$-S) have larger soma size than $\beta$-MNs $(\beta-\mathrm{d}, \beta$-s) and $\gamma$-MNs $(\gamma$-d, $\gamma$-s). 2$) \alpha$-MNs $(\alpha-$ $\mathrm{FF}, \alpha$-FR, $\alpha$-S) have a high density of vGlut1 synaptic varicosities from Ia sensory afferent innervation, whereas $\gamma$-MNs $(\gamma$-d, $\gamma$-s) essentially lack this innervation. 3) $\alpha$-MNs $(\alpha-\mathrm{FF}, \alpha$-FR, $\alpha-S)$ innervate extrafusal fibers, $\beta$-MNs $(\beta-\mathrm{d}, \beta$-s) innervate extrafusal fibers and intrafusal fibers. While $\gamma-$ MNs $(\gamma-\mathrm{d}, \gamma$-s) innervate intrafusal fibers. 4) comparison of biophysical properties between $\alpha$-MN versus $\gamma$-MNs: input resistance ( $\alpha$-MN $>\gamma$-MNs), rheobase ( $\alpha$-MN $>\gamma$-MNs), firing frequency ( $\alpha-M N$ $<\gamma$-MNs), AHP half-decay and duration ( $\alpha$-MN versus $\gamma$-MNs is variable). 5) $\alpha$-MNs express different molecular correlates than $\gamma$-MNs. 6) $\alpha$-MNs generate force during movement, while $\gamma$-MNs regulate the muscle spindle (MS) sensitivity, which is important for muscle reflexes and muscle proprioception during movement. The biophysical properties described in this table are relative patterns that vary according to species and developmental stage of the animal. (Adapted from Kanning et al., 2010 and Manuel and Zytnicki, 2011 and various studies cited in main text)

\subsection{Nuclear receptors (NRs)}

Multicellular organisms use nuclear receptors (NRs) to regulate gene expression pathways when encountering signals that emerge from the environment, intracellular states, or during normal development. They regulate NR function through three mechanistic pathways in cells: 1) binding to a lipophilic ligand, 2) covalent modifications like phosphorylation, and 3) protein-protein interactions (Giguère, 1999). NR family proteins have a general structure with four regions that allow them to influence gene expression: 1) a modulator domain, 2) a DNA-binding domain (DBD), 3) a hinge region and 4) a ligandbinding domain (LBD) (Fig. 1.10A). Transcriptional activation is carried out in the activation function-1 (AF-1) region within the modulator domain (Giguère, 1999). Diverse NR isoforms with specific modulator domains can be expressed, which allows the organism to target specific biological functions (Giguère, 1999). The DBD is highly conserved in NRs and this region contains two zinc finger modules that bind unique DNA sequences known as hormone response elements (HREs) (Giguère, 1999). The highly variable and malleable hinge region is used to connect the DBD with the LBD and also permits various orientations for the DBD to bind HRE regions, assisting dimerization of NRs (Glass, 1994; Giguère, 1999). Finally, the LBD regulates various facets of NR functions: ligand binding, nuclear localization, dimerization, and transactivation, amongst others (Giguère, 1999). LBD domain contains a conserved region called activation function-2 (AF-2) that enables transactivation mechanisms through coactivators (Giguère, 1999).

Classically, certain steroid receptors translocate to the nucleus to initiate gene expression when bound by a specific ligand (Pratt and Toft, 1997; Giguère, 1999). However, the majority of NRs regulate gene expression by constitutive nuclear localization and in a ligand-independent manner (Horlein et al., 1995; Chen and Evans, 1995; Sap et al., 1989; 
Giguère, 1999). NRs interact with transcription, coactivators, or corepressors to regulate gene expression (Horwitz et al., 1996; Beato and Sanchez-Pachecho, 1996; Freedman, 1999; Mckenna et al., 1999; Glass et al., 1997; Giguère, 1999).

The nuclear receptor (NR) superfamily subdivision NR group 3 (NR3) has several members: androgen receptors (AR), estrogen receptors (ERs), glucocorticoids receptors (GR), mineralocorticoid receptors (MR), progesterone receptors (PR) and estrogen-related receptors (ERRs) (Fig. 1.10B) (see Giguère, 2002; Tremblay and Giguère, 2007 for review). A subdivision within NR3 is the NR group 3A (NR3A), which includes estrogen receptors (ERs), ER $\alpha$ (NR3A1) and ER $\beta$ (NR3A2) that are structurally and functionally related to the NR group 3B (NR3B) subfamily known as the estrogen-related receptors (ERRs) (Giguère, 2002; Benoit et al., 2006). There are three members in the ERRs subfamily: 1) ERR $\alpha$ (NR3B1, ERR1, ESRRA), 2) ERR $\beta$ (NR3B2, ERR2, ESRRB), 3) ERR $\gamma$ (NR3B3, ERR3, ESRRG) (Fig. 1.10B) (Tremblay and Giguère, 2007; Giguère, 2002).

\subsubsection{Estrogen-related receptor (ERR) family structural elements and phylogeny}

Estrogen-related receptors (ERRs) are a subfamily of orphan nuclear receptors (ONRs), which play many roles in development and disease states and have a high sequence homology (Fig. 1.10C) (Giguère, 2002; Hong et al., 1999). Unlike the NRs for cortisol, aldosterone, estradiol, testosterone, and others, which have a known ligand, ONRs generally do not have a known ligand (Giguère, 1999). However, ONRs, like the NR superfamily, contain a highly conserved DNA-binding domain (DBD) and ligand-binding domain (LBD), but they lack regions such as the AF-2 (Giguère, 1999). It is hypothesized that ONRs thereby lost their ability to bind ligands through evolution; however, they still possess the LBD for other functions like coactivator interactions and dimerization (Giguère, 1999).

A) Representation of general gene structure of nuclear receptors (NRs)

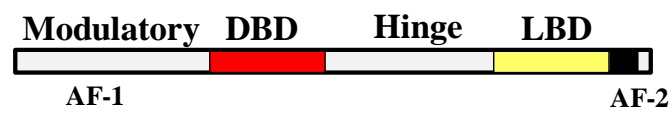

B) Representation of group III nuclear receptor (NR) phylogenetic tree

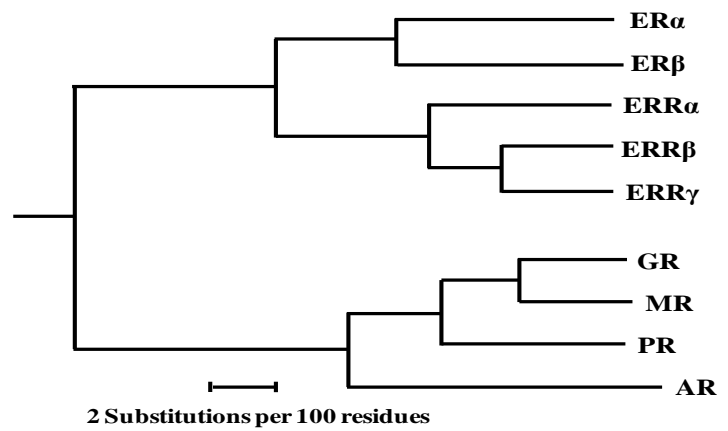


C) Representation of estrogen-related receptor (ERR) structural elements, a subfamily of orphan nuclear receptors (ONRs)

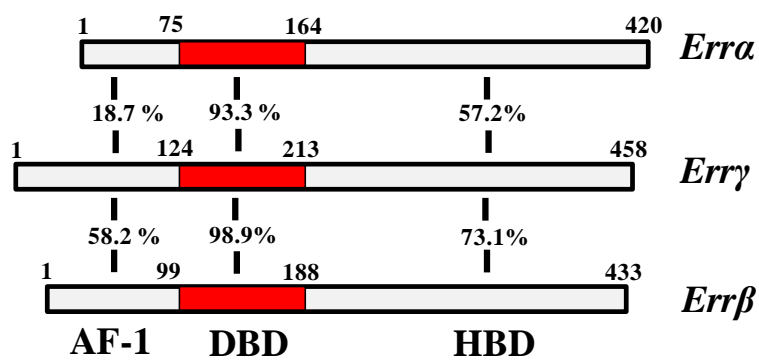

Figure 1.10 Schematic representation of nuclear receptor (NR) structure, group III nuclear receptor (NR3) phylogenetic tree and estrogen-related receptor (ERR) structural elements

A) The general structural domains of nuclear receptors (NRs) contain four different regions: 1) modulator domain containing the activation function-1 (AF-1) regions (transcriptional activation domain), 2) DNA-binding domain (DBD) that recognizes and binds DNA, 3) hinge region connects the DBD to the LBD, 4) the LBD binds specific ligands, which contains the activation function-2 (AF-2) motif for ligand-dependent transactivation by interactions with specific cofactors. (Adapted from Giguère,1999) B) A phylogenetic tree group III NRs (adapted from Giguère, 2002). C) Homologous regions of ERR, a subfamily of orphan nuclear receptors (ONRs) in mice depicting shared sequence similarity (in the percentage of amino acids) in major domains. (Adapted from Hong et al., 1999)

\subsubsection{Estrogen-related receptor 1 (Err1)}

Estrogen-related receptor 1 (Err1) is expressed in various tissues including bone tissue, brown and white adipose tissue, cartilage tissue, heart, intestine, liver, macrophages, skeletal muscle and breast cancer cells (Tremblay and Giguère, 2007). Errl KO mice generated by Luo et al., (2003), showed decreased peripheral fat accumulation and body weight. Furthermore, microarray studies using adipose tissue from Errl KO mice showed a shift in the gene expression pattern of enzymes that regulate fat catabolism versus fat synthesis (Luo et al., 2003). Err1 and estrogen-related receptor 3 (Err3) target similar promoter regions and are essential for regulating metabolic genes involved in heart function (Dufour et al., 2007). Specifically, Errl KO mice undergo heart failure due to the abnormal signaling of a metabolic pathway that is normally used when dealing with hemodynamic stressors (Huss et al., 2007). Err1 signaling is involved in several important pathways required for biological functions such as heart function, fat metabolism, thermogenesis and innate immunity (Tremblay and Giguère, 2007).

\subsubsection{Estrogen-related receptor 2 (Err2)}

Estrogen-related receptor (Err2) is expressed among other tissues in the placenta and inner ear and is important for their development (Luo et al., 1997; Pettersson et al., 1996; 
Tremblay and Giguère, 2007). For instance, Err2 conditional KO mutant mice display a decreased quantity of endolymph-producing cells in the cochlea, utricle, and ampulla resulting in abnormal fluid homeostasis in the inner ear, which affects hearing perception (Chen and Nathans, 2007). In addition, endolymph-producing strial marginal cells in the cochlea of Err2 KO mutant mice change their cellular fate to become epithelial cells and show loss of $\mathrm{K}^{+}$ion channels and transporter proteins (Chen and Nathans, 2007). Thus, Err2 controls $\mathrm{K}^{+}$ion channel expression in the inner ear. Furthermore, humans with mutations in ERR2 are correlated with hearing loss (Collin et al., 2008). In summary, these studies revealed that Err2 has an integral role in regulating cell fate during development.

\subsubsection{Estrogen-related receptor 3 (Err3)}

Estrogen-related receptor (Err3) is expressed in the spinal cord, heart, inner ear and breast cancer cells (Friese et al., 2009; Tremblay and Giguère, 2007). Similarities in Err1 and Err3 DNA binding sites and protein interaction suggested that they may have a similar role in the heart and mitochondria (Willy et al., 2004; Huss and Kelly, 2004; Alaynick et al., 2007). Furthermore, studies have suggested that they both bind promoter sequences that are also recognized by nuclear respiratory factor 1 (Nrf-1), cAMP responsive element binding protein (Creb) and signal transducer and activator of transcription (Stat3) (Dufour et al., 2007). Moreover, studies by Alaynick et al., (2007), have shown that Err3 KO mutant mice show abnormal electrocardiogram (ECG) patterns, thus, Err3 is essential for expression of genes needed for metabolic and mitochondrial processes involved in the fetal heart (Alaynick et al., 2007). Studies by Friese et al., (2009), demonstrated that Err3 is a molecular correlate for $\gamma$ MNs that is highly expressed in relatively small soma size MNs in mice at postnatal day 14 (P14). Studies by Nolan et al., (2013), have shown that age-related hearing loss (ARHL) in women is correlated with rs2818964 single-nucleotide polymorphism (SNP) in the ESRRG gene. Furthermore, Err3 KO mutant mice showed a moderate hearing loss and this deficiency affected female mice more than males, which is a phenotype correlated with human London ARHL cohort (Nolan et al., 2013).

\subsection{Rationale and Objectives}

Studies have shown that morphogen gradients secreted during development lead to the expression of transcription factors that regulate the initial organization $\mathrm{MN}$ positional identities in the embryonic spinal cord (Tanabe et al., 1998; Briscoe et al., 2000; Jessell, 2000). However, the molecules and their mechanistic actions driving MN functional 
diversification in the spinal motor "pools" into alpha and gamma subtypes ( $\alpha$ - and $\gamma$ subtypes) remain largely unresolved. Furthermore, previous studies have shown that Egr3 KO mutant mice did not develop muscle spindles (MSs), which resulted in abnormal gait patterns, thus, proprioceptive information from MSs is important for motor behavior in vivo (Tourtellotte and Milbrandt, 1998; Akay et al., 2014). $\gamma$-MNs directly control intrafusal fibers within the MSs and studies have implicated them in voluntary movements, reflexes and muscle tone (see Murthy, 1978 for review). Still, studies have yet to characterize the molecular pathways that determine $\gamma$-MN biophysical properties that allow them to control gait, posture and precision movements.

Quantitative immunodetection studies were performed using P5 spinal cord cryosections from wild-type mice by Lee, (2013), which showed that relatively small soma size cells expressed Err2 while lacking RNA-binding protein, fox-1 homolog 3 (Rbfox3, $\mathrm{NeuN}$ ) expression. However, behavior studies in Err2 conditional KO mice did not show significant differences when challenged with motor behavior and force generation tasks when compared to control mice (Lee, 2013). Moreover, previous studies had shown that Err3, a paralogue of Err2, is expressed in small soma size putative $\gamma$-MNs and thus, is a $\gamma$-MN molecular correlate (Friese et al., 2009). Thus, I hypothesized that Err2 and Err3 are redundantly expressed transcription factors that determine the functional properties of $\gamma$ MNs, which are integral to the regulation of gait, posture and precision movements in mice.

\section{This Ph.D. work had five aims:}

Aim I: To resolve Err2 and Err3 expression pattern during early postnatal development through quantitative immunodetection studies.

Aim II: To use in ovo electroporation technique express Err2 and Err3 in chick to evaluate their influence on $\mathrm{MN}$ properties.

Aim III: To generate Err2 $2^{\text {floxflox}}$; Err3 ${ }^{\text {floxflox}}$; $\mathrm{ChAT}^{\text {Cre }}$ conditional knockout $\left(E r r 2 / 3^{c K O}\right)$ mice and analyze $\mathrm{MN}$ biophysical properties using whole cell patch-clamp recordings.

Aim IV: To examine the consequences of altering $\gamma$-MN function in $E r r 2 / 3^{c K O}$ mice on gait and precision movements.

Aim V: To take steps towards identifying molecular mechanisms through which Err2 and Err3 may regulate the biophysical properties of $\gamma$-MNs. 


\section{MATERIALS \& METHODS}

\subsection{Laboratory consumables and glassware/plasticware}

Several types of glassware and plasticware were used: ISOLAB Laborgeräte GmbH, Kavalierglass, a.s., Brand GmbH + CO KG, Eppendorf Vertrieb Deutschland, Starlab GmbH, Sarstedt AG \& Co. The dissection tools for chicken embryos and mice were purchased from Fine Science Tools Inc.

\subsection{Antibodies}

Table 2.1 Primary Antibodies

\begin{tabular}{lllll}
\hline Antigen & Host Species & Dilution & Supplier & Cat. \# \\
\hline DsRed & Rabbit & $1: 1000$ & Clonetech & $632496 ?$ \\
Err2 & Mouse & $1: 4000$ & R\&D Systems & PP-H6705-00 \\
Err3 & Mouse & $1: 1000$ & R\&D Systems & PP-H6812-00 \\
GFP & Chicken & $1: 2000$ & Abcam & ab13970 \\
Kcna10 & Rabbit & $1: 250$ & Aviva Systems Bio. & ARP35178_T100 \\
NeuN & Mouse & $1: 1500$ & Merck Millipore & MAB377 \\
vACht & Rabbit & $1: 500$ & Synaptic Systems & 139103 \\
vGlut1 & Guinea Pig & $1: 1000$ & Merck Millipore & AB5905 \\
\hline
\end{tabular}

Table 2.2 Secondary Antibodies

\begin{tabular}{|c|c|c|c|c|c|}
\hline Target & Conjugate & $\begin{array}{l}\text { Host } \\
\text { Species }\end{array}$ & Dilution & Supplier & Cat. \# \\
\hline Chicken IgG & Alexa Fluor-488 & Goat & $1: 2000$ & Thermo Fisher Scientific & A-11039 \\
\hline $\begin{array}{l}\text { Guinea Pig } \\
\text { IgG }\end{array}$ & Alexa Fluor-488 & Goat & $1: 2000$ & Thermo Fisher Scientific & A-11073 \\
\hline $\begin{array}{l}\text { Mouse } \\
\text { IgG2a }\end{array}$ & Alexa Fluor-488 & Goat & $1: 2000$ & Thermo Fisher Scientific & A-21131 \\
\hline $\begin{array}{l}\text { Mouse } \\
\text { IgG2b }\end{array}$ & Alexa Fluor-555 & Goat & $1: 2000$ & Thermo Fisher Scientific & A-21147 \\
\hline Mouse IgG1 & Alexa Fluor-647 & Goat & $1: 2000$ & Thermo Fisher Scientific & A-21240 \\
\hline Mouse IgG1 & DyLight-405 & Goat & $1: 250$ & $\begin{array}{l}\text { Jackson ImmunoResearch } \\
\text { Inc. }\end{array}$ & $\begin{array}{l}115-475- \\
205\end{array}$ \\
\hline Rabbit IgG & Alexa Fluor-555 & Goat & $1: 2000$ & Thermo Fisher Scientific & A-32732 \\
\hline Rabbit IgG & Alexa Fluor-647 & Goat & $1: 2000$ & Thermo Fisher Scientific & A-32733 \\
\hline
\end{tabular}

\subsection{Chemicals}

Table 2.3 Chemicals and Supplier

\begin{tabular}{ll}
\hline Chemical & Supplier \\
\hline Adenosine 5'-triphosphate magnesium salt & Sigma-Aldrich Chemie GmbH \\
Agar-Agar & Carl Roth $\mathrm{GmbH}$ \\
Ampicillin & Carl Roth $\mathrm{GmbH}$ \\
\hline
\end{tabular}




\begin{tabular}{|c|c|}
\hline Agarose NEEO & Carl Roth GmbH \\
\hline Bovine serum albumin fraction V (BSA) & Carl Roth GmbH \\
\hline Calcium Chloride dihydrate & Carl Roth GmbH \\
\hline Choline bicarbonate & Sigma-Aldrich Chemie GmbH \\
\hline Cholera toxin B subunit Alexa conjugated & Invitrogen $\mathrm{GmBH}$ \\
\hline dNTP mix & Fermentas GmbH \\
\hline Dimethyl sulfoxide (DMSO) & Sigma-Aldrich Chemie GmbH \\
\hline Ethanol $99.9 \%$ & CHEMSOLUTE \\
\hline Ethidium Bromide 1\% & Carl Roth GmbH \\
\hline Ethlenediaminetetraacetate (EDTA) & Carl Roth GmbH \\
\hline Ethyl Pyruvate & Sigma-Aldrich Chemie GmbH \\
\hline Ethyleneglycoltetraacetic acid (EGTA) & Sigma-Aldrich Chemie GmbH \\
\hline Fluoro-gold & Fluorochrome, LLC \\
\hline Gelatin & Fluka Analytical \\
\hline GeneRuler 1 kb DNA ladder & Thermo Fisher Scientific GmbH \\
\hline Glucose Anhydrous & Carl Roth GmbH \\
\hline Glycerol & Carl Roth GmbH \\
\hline Guanosine 5'-triphosphate sodium salt hydrate & Sigma-Aldrich Chemie GmbH \\
\hline HEPES & Sigma-Aldrich Chemie GmbH \\
\hline Isopropanol & Carl Roth GmbH \\
\hline Kynurenic acid & Sigma-Aldrich Chemie GmbH \\
\hline L-Glutathione reduced & Sigma-Aldrich Chemie GmbH \\
\hline Loctite 401 & Henkel Adhesive North America \\
\hline L-15 Medium Leibowitz & Applichem $\mathrm{GmbH}$ \\
\hline Magnesium Chloride & Carl Roth GmbH \\
\hline Magnesium sulfate heptahydrate & Carl Roth GmbH \\
\hline myo-Inositol & Sigma-Aldrich Chemie GmbH \\
\hline Orange-G & Applichem GmbH \\
\hline Paraformaldehyde (PFA) & Carl Roth GmbH \\
\hline PBS pH 7.2 & Thermo Fisher Scientific GmbH \\
\hline Phosphocreatine di(tris) salt & Sigma-Aldrich Chemie GmbH \\
\hline Potassium Chloride & Carl Roth GmbH \\
\hline Potassium Dihydrogen phosphate & Carl Roth GmbH \\
\hline Potassium Methane Sulfonate & Sigma-Aldrich Chemie GmbH \\
\hline Potassium gluconate & Sigma-Aldrich Chemie GmbH \\
\hline Proteinase K & Applichem GmbH \\
\hline RNAse Away & Molecular Bioproducts \\
\hline QIAzol Lysis Reagent & Qiagen \\
\hline Sodium Chloride & Carl Roth GmbH \\
\hline
\end{tabular}




\begin{tabular}{ll}
\hline Sodium Dihydrogen phosphate dihydrate & Carl Roth $\mathrm{GmbH}$ \\
Sodium dodecyl sulfate (SDS) & Carl Roth $\mathrm{GmbH}$ \\
\hline Sodium Hydrogen Carbonate & Carl Roth GmbH \\
Sodium L-ascorbate & Sigma-Aldrich Chemie GmbH \\
Sucrose & Carl Roth GmbH \\
Tissue-Tek O.C.T. embedding medium & Sakura Fintek GmbH \\
Tris & Carl Roth GmbH \\
Triton X-100 & Carl Roth $\mathrm{GmbH}$ \\
Trizol reagent & Invitrogen $\mathrm{GmBH}$ \\
Yeast extract & Applichem $\mathrm{GmbH}$ \\
\hline
\end{tabular}




\section{$2.4 \quad$ Enzymes}

Table 2.4 Enzymes and Supplier

\begin{tabular}{ll}
\hline Enzyme & Supplier \\
\hline DreamTaq ${ }^{\text {TM }}$ Green Taq DNA polymerase & Thermo Fisher Scientific GmbH \\
DNA Ligation Kit & Takara Bio Inc. \\
Phusion High Fidelity DNA polymerase & New England Biolabs Inc. \\
Restriction Enzymes & Thermo Fisher Scientific GmbH \\
Shrimp Alkaline Phosphatase & Thermo Fisher Scientific GmbH \\
SP6 RNA Polymerase & Roche Diagnostics GmbH \\
T4 DNA Ligase & Thermo Fisher Scientific GmbH \\
T4 Polynucleotide Kinase & Thermo Fisher Scientific GmbH \\
T7 RNA Polymerase & Roche Diagnostics GmbH \\
\hline
\end{tabular}

\section{$2.5 \quad$ Kits}

Table 2.5 Kits and Supplier

\begin{tabular}{|c|c|}
\hline Kit & Supplier \\
\hline GeneJET Gel Extraction Kit & Thermo Fisher Scientific GmbH \\
\hline GeneJet Plasmid Miniprep Kit & Thermo Fisher Scientific GmbH \\
\hline iScript cDNA Synthesis Kit & Bio-Rad GmbH \\
\hline Plasmid Maxi Kit & Qiagen $\mathrm{GmbH}$ \\
\hline PrimeScript $1^{\text {st }}$ strand cDNA synthesis Kit & Takara Bio Inc. \\
\hline Qubit dsDNA HS Assay Kit & Thermo Fisher Scientific GmbH \\
\hline Transcriptor High Fidelity cDNA synthesis kit & Roche Life Science \\
\hline TruSeq RNA Sample Preparation v2 Kit & Illumina $^{\mathrm{R}}$ \\
\hline TruSeq SBS Kit v3-HS & Illumina $^{\mathrm{R}}$ \\
\hline TruSeq SR Cluster Kit v3-cBot-HS & Illumina ${ }^{\mathrm{R}}$ \\
\hline
\end{tabular}

\subsection{Plasmids}

Table 2.6 Plasmids

\begin{tabular}{ll}
\hline Plasmid & Supplier/Reference \\
\hline GATA2-GFP & Zhou et al., 2000 \\
\hline Hb9-GFP & Lee et al., 2004 \\
pCAGEN & Addgene Inc. \\
pGK-Cre & Addgene Inc. \\
pCRII-TOPO & Invitrogen GmbH \\
pCAGGS-T2TP & Gift from K. Kawakami (Sato et al., 2007) \\
\hline pT2K-CAGGS-EGFP & Sato et al., 2007 \\
\hline
\end{tabular}




\subsection{Programs \& Databases}

Table 2.7 Software and Supplier

\begin{tabular}{|c|c|c|}
\hline Software/Database & Application & Supplier \\
\hline $\begin{array}{l}\text { Adobe Illustrator } \\
\text { CS5.1 }\end{array}$ & Image processing & Adobe Inc. \\
\hline $\begin{array}{l}\text { Adobe Photoshop } \\
\text { CS5.1 }\end{array}$ & Image processing & Adobe Inc. \\
\hline ApE & Vector construction & M. Wayne Davis \\
\hline $\begin{array}{l}\text { DigiGait Imager } \\
\text { and Analysis } \\
\text { software }\end{array}$ & Animal gait analysis & Mouse Specifics Inc. \\
\hline ECR Browser & $\begin{array}{l}\text { Promotor/Enhancer } \\
\text { analysis }\end{array}$ & ecrbrowser.dcode.org/ \\
\hline $\begin{array}{l}\text { FastQC quality } \\
\text { control tool } \\
\text { Version } \mathbf{0 . 1 0 . 1}\end{array}$ & $\begin{array}{l}\text { Quality control tool } \\
\text { for high throughput } \\
\text { sequencing data }\end{array}$ & $\begin{array}{l}\text { http://www.bioinformatics.babraham.ac.uk/projects/fas } \\
\text { tqc }\end{array}$ \\
\hline $\begin{array}{l}\text { GoPro Studio } \\
\text { Version 2.5.4 }\end{array}$ & GoPro video editing & GoPro, Inc. \\
\hline GraphPad Prism & Data analysis & GraphPad Software, Inc. \\
\hline ImageJ/FIJI & Image processing & Abramoff et al., 2004 \\
\hline Imaris 8.0 & $\begin{array}{l}\text { Microscopy Image } \\
\text { analysis software }\end{array}$ & Bitplane, AG \\
\hline JASPAR CORE & $\begin{array}{l}\text { Transcription factor } \\
\text { binding sites database }\end{array}$ & jaspar.genereg.net \\
\hline pDraw & Vector construction & AcaClone Software \\
\hline $\begin{array}{l}\text { proDAD Defishr } \\
\text { Version } 1.0\end{array}$ & $\begin{array}{l}\text { Video fisheye } \\
\text { correction }\end{array}$ & proDAD GmbH \\
\hline R software & Statistical computing & http://www.R-project.org/. \\
\hline
\end{tabular}




\subsection{Solutions}

Table 2.8 Solutions

\begin{tabular}{|c|c|}
\hline Solutions & Reagents \\
\hline 4\% Agarose & $4 \%$ agarose in RaCSF, spinal cord support for dissection \\
\hline $20 \%$ Gelatin solution & $\begin{array}{l}20 \% \text { gelatin in RaCSF, spinal cord embedding mold for } \\
\text { dissection }\end{array}$ \\
\hline Anesthetic Solution & $\begin{array}{l}100 \mathrm{mg} / \mathrm{kg} \text { Ketamine, } 20 \mathrm{mg} / \mathrm{kg} \text { Xylazine in PBS } 7.2 \text {; volume } \\
\text { administered: } 0.10 \mathrm{ml} / 10 \mathrm{~g} \text { body weight }\end{array}$ \\
\hline Antibody Staining Solution & $1 \mathrm{xPBS}$ pH $7.2,1 \%$ or $2.5 \%$ BSA; $0.5 \%$ or $0.25 \%$ Triton $\mathrm{X}-100$ \\
\hline DNA Running Buffer & $8 \%$ Sucrose in TAE; $1 \mathrm{mg} / \mathrm{ml}$ Orange $\mathrm{G}$ \\
\hline $\begin{array}{l}\text { Fluoro-Gold (FG) Tracer } \\
\text { Solution }\end{array}$ & $\begin{array}{l}0.5 \%(\mathrm{w} / \mathrm{v}) \text { in } 1 \mathrm{x} \text { PBS } \mathrm{pH} 7.2 \text {; volume administered: } 0.10 \mathrm{ml} / 5 \mathrm{~g} \\
\text { body weight }\end{array}$ \\
\hline LB-Agar & LB medium with $1 \%(\mathrm{w} / \mathrm{v})$ agar-agar \\
\hline LB-Medium & $5 \mathrm{~g} / \mathrm{l} \mathrm{NaCl}, 5 \mathrm{~g} / \mathrm{l}$ yeast extract, $10 \mathrm{~g} / \mathrm{l}$ tryptone \\
\hline Mouse tail biopsy Lysis Buffer & $\begin{array}{l}30 \mathrm{mM} \mathrm{NaCl}, 0.1 \mathrm{M} \text { Tris } \mathrm{pH} 8.0,0.2 \mathrm{M} \text { EDTA, } 0.05 \% \text { SDS, } 0.5 \\
\mathrm{mg} / \mathrm{ml} \text { Proteinase K}\end{array}$ \\
\hline $\begin{array}{l}\text { Chick dissecting and recording } \\
\text { artificial cerebrospinal fluid } \\
\text { (DaCSF) and (RaCSF) (in } \mathbf{m M}) \text { : }\end{array}$ & $\begin{array}{l}139 \mathrm{NaCl}, 3 \mathrm{KCl}, 1 \mathrm{MgCl}_{2}, 17 \mathrm{NaHCO}_{3}, 12.2 \text { dextrose, } 3 \mathrm{CaCl}_{2} \text {. } \\
\text { The solution pH was } \sim 7.3 \text { and the osmolality was } 315 \\
\text { mosmol/kgH } \mathrm{O} \text { (adjusted using sucrose). }\end{array}$ \\
\hline $\begin{array}{l}\text { Chick intracellular pipette } \\
\text { solution (in mM): }\end{array}$ & $\begin{array}{l}130 \mathrm{MeSO}_{3} \mathrm{H}, 10 \mathrm{KCl}, 2 \mathrm{MgCl}_{2}, 0.4 \mathrm{EGTA}-\mathrm{KOH}, 0.1 \mathrm{CaCl}_{2}, 10 \\
\mathrm{HEPES}, 2 \mathrm{ATP}-\mathrm{MG}^{2+} \text { salt, } 0.4 \mathrm{GTP}-\mathrm{Na}^{+} \text {salt. The solution } \mathrm{pH} \\
\text { was } 7.3 \text { (adjusted with } \mathrm{KOH} \text { ) and the osmolality was } 300 \\
\text { mosmol/ } / \mathrm{kgH}_{2} \mathrm{O} \text { (adjusted using sucrose) and contained } 25 \mu \mathrm{M} \\
\text { Alexa fluor } 568 \text {. }\end{array}$ \\
\hline $\begin{array}{l}\text { Mouse intracellular pipette } \\
\text { solution }(\mathbf{m M}) \text { : }\end{array}$ & $\begin{array}{l}131 \mathrm{~K} \text {-methanesulfonate }\left(\text { or } \mathrm{MeSO}_{3} \mathrm{H}\right), 6 \mathrm{NaCl}, 0.1 \mathrm{CaCl}_{2}, 1.1 \\
\text { EGTA-KOH, } 10 \mathrm{HEPES}, 0.3 \mathrm{MgCl}_{2}, 3 \mathrm{ATP}-\mathrm{MG}^{2+} \text { salt, } 0.5 \mathrm{GTP}- \\
\mathrm{Na}^{+} \text {salt, } 2.5 \mathrm{~L} \text {-glutathione reduced, } 5 \text { phosphocreatine di(tris) } \\
\text { salt. The solution pH was } 7.25 \text { (adjusted with } \mathrm{KOH} \text { ) and the } \\
\text { osmolality was } 300 \mathrm{mosmol} / \mathrm{kgH}_{2} \mathrm{O} \text { (adjusted using sucrose). }\end{array}$ \\
\hline $\begin{array}{l}\text { Mouse dissecting artificial } \\
\text { cerbrospinal fluid (DaCSF) (in } \\
\text { mM): }\end{array}$ & $\begin{array}{l}191 \text { sucrose, } 0.75 \mathrm{~K} \text {-gluconate, } 1.25 \mathrm{KH}_{2} \mathrm{PO}_{4}, 26 \text { choline } \\
\text { bicarbonate }\left(80 \% \text { solution), } 4 \mathrm{MgSO}_{4}, 1 \mathrm{CaCl}_{2}, 20 \text { dextrose, } 2\right. \\
\text { kynurenic acid, } 1 \text { (+)-sodium L-ascorbate, } 5 \text { ethyl pyruvate, } 3 \\
\text { myo-Inositol. The solution pH was } ~ 7.3 \text { while incubated in } 95 \% \\
\mathrm{O}_{2}-5 \% \mathrm{CO}_{2} \text { (carbogen) and the osmolality was } 310 \\
\text { mosmol/kgH } \mathrm{kg}_{2} \mathrm{O} \text { (adjusted using sucrose). }\end{array}$ \\
\hline $\begin{array}{l}\text { Mouse recording artificial } \\
\text { cerebrospional fluid (RaCSF) (in } \\
\text { mM): }\end{array}$ & $\begin{array}{l}121 \mathrm{NaCl}, 3 \mathrm{KCl}, 1.25 \mathrm{NaH}_{2} \mathrm{PO}_{4}, 25 \mathrm{NaHCO}_{3}, 1.1 \mathrm{MgCl}_{2}, 2.2 \\
\mathrm{CaCl}_{2}, 15 \text { dextrose, } 1 \text { (+)-sodium L-ascorbate, } 5 \text { ethyl pyruvate, } 3 \\
\text { myo-Inositol. The solution pH was } \sim 7.4 \text { while incubated in } 95 \% \\
\mathrm{O}_{2}-5 \% \mathrm{CO}_{2} \text { (carbogen) and the osmolality was } 310 \\
\text { mosmol/kgH } \mathrm{kg} \text { (adjusted using sucrose). }\end{array}$ \\
\hline
\end{tabular}

\subsection{Animal care}

Mice were housed and cared for by the European Neuroscience Institute (ENI) animal facility at Georg-August-Universität Göttingen. Animal experiments were approved by institutions abiding German federal and state regulations.

\subsection{Mouse interbreeding and mouse genotyping}

For obtaining transgenic and conditional knockout (cKO) mice, the Cre/loxP system was used. In coliophage P1, the Cre recombinase enzyme is required for DNA recombination (Sauer and Hendersen, 1988). In mammalian cells, DNA recombination is utilized in normal 
and abnormal cellular states, thus, the Cre/loxP system can used for site-specific recombination events allowing for manipulation of the genome (Sauer and Hendersen, 1988). Transgenic mice expressing Cre recombinase under a specific promoter can be bred with mice that have an exon of interest in a gene floxed by two loxP sites resulting in offspring that have this region excised in specific cells (Hall et al., 2009). A similar system called Flp/FRT derived from Saccharomyces cervisiae can be employed to specifically manipulate the genome as well (Hall et al., 2009).

ChAT-IRES-Cre mouse line with an internal ribosome entry sequence-Cre recombinase (IRES-Cre) fused insertion following choline acetyltransferase (ChAT) gene stop codon was used to specifically express Cre recombinase in cholinergic neurons, such as MNs (Fig. 2.1A, B) (Rossi et al., 2011; Jackson Laboratories).

A)

W.T.

Chat allele

Chromosome 14, exon 15

Targeted

Chat allele

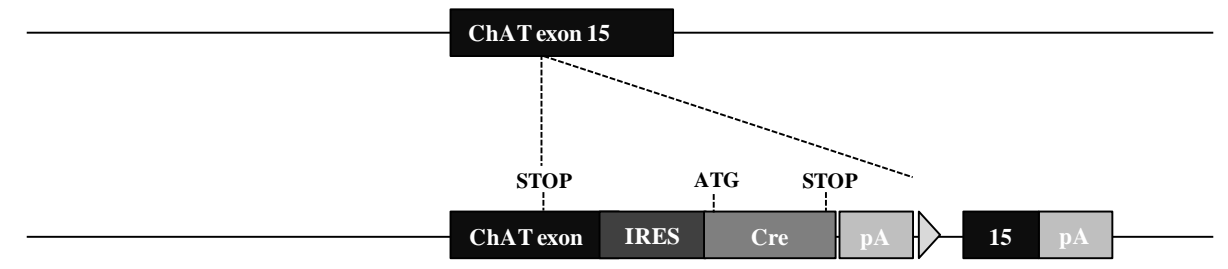

B)

ChAT-IRES-Cre

Knockin

Chat promoter

ChAT exon IRES Cre

(Chat ${ }^{\text {Cre }}$ mice)

\section{Figure 2.1 Schematic diagram of $\mathrm{Chat}^{\mathrm{Cre}}$ mouse line}

A) A homologous DNA recombination event was used to insert IRES fused to Cre recombinase downstream of the Chat gene stop codon. B) The Chat ${ }^{\mathrm{Cre}}$ mice express Cre recombinase under the endogenous Chat promoter in all cholinergic neurons, thus, allowing for the targeting of Cre recombinase expression in MNs. (Adapted from Rossi et al., 2011)

To generate the Chat::Tomato mouse line, a mouse line with a ubiquitously expressing Rosa26 locus was altered to increase expression by inserting a construct containing the $C A G$ promoter (potent and ubiquitous) and a loxP-flanked ('floxed') stop cassette that regulates the expression of the tdTomato fluorescent protein (Zong et al., 2005; Muzumdar et al., 2007; Madisen et al., 2010; Jackson Laboratories). Moreover, mRNA stability was increased by inserting a woodchuck hepatitis virus post-transcriptional regulatory element (WPRE) (Zuffrey et al., 1999; Madisen et al., 2010). The floxed STOP Rosa26-Cag-LSL-tdTomato-WPRE mouse line was interbred with the Chat ${ }^{\text {Cre }}$ mouse line 
resulting in offspring that showed the expression of tdTomato fluorescence in cholinergic cells, including MNs (Fig. 2.2A, B). The Chat::Tomato mice were both viable and fertile.

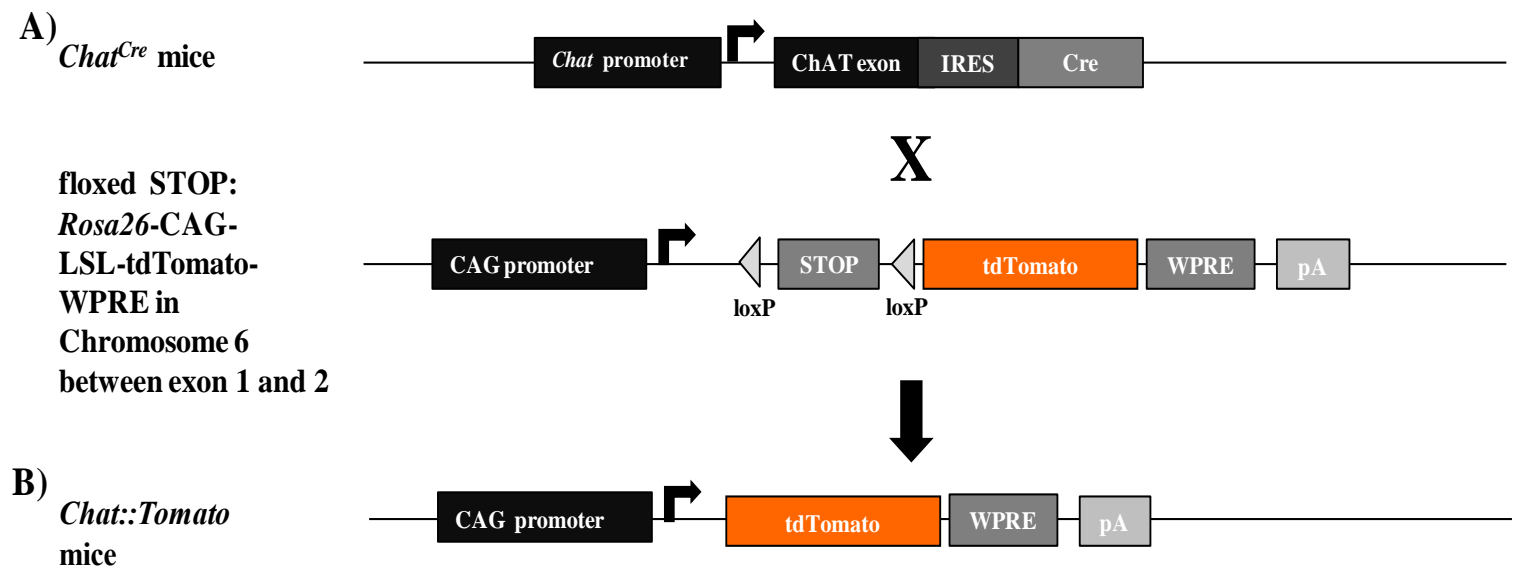

Figure 2.2 The interbreeding strategy for generating Chat::Tomato mice

A) $\mathrm{Chat}^{\mathrm{Cre}}$ mice were interbred with floxed STOP Rosa26-Cag-LSL-tdTomato-WPRE mice, which resulted in offspring with a deletion of the STOP casette leading to the expression of Cre recombinase in their cholinergic neurons. B) Chat::Tomato mice express tdTomato (DsRed fluorescent protein variant) in MNs. (Adapted from Madisen et al., 2010)

To generate conditional knockout mice lacking Err2 and Err3 expression in MNs, floxed Esrrb ${ }^{\text {loxp/loxp }}$ and $C h a t^{\text {Cre }}$ mouse line was interbred with floxed Esrrg ${ }^{\text {loxp/loxp }}$ mouse line (Fig. 2.3A, B) (Chen and Nathans, 2007; Institut Clinique de la Souris (ICS), Alsace, France; Jackson Laboratories, Inc.). The resulting Esrrb ${ }^{\text {floxflox }}$ Esrrg ${ }^{\text {floxfflox }}$; Chat ${ }^{\text {Cre }}$ conditional knockout (Err2/3 ${ }^{c K O}$ ) offspring were both viable and fertile (Fig. 2.3B). 
A)

Chat ${ }^{\text {Cre }}$

floxed $E s r r b$ allele: LoxP sites flanking Chromosome 12, exon 2 containing Esrrb gene

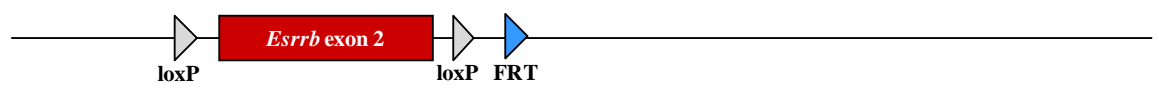

\section{$\mathbf{X}$}

floxed $\operatorname{Esrr}$ allele: LoxP sites flanking Chromosome 1, exon 2 containing Esrrg gene
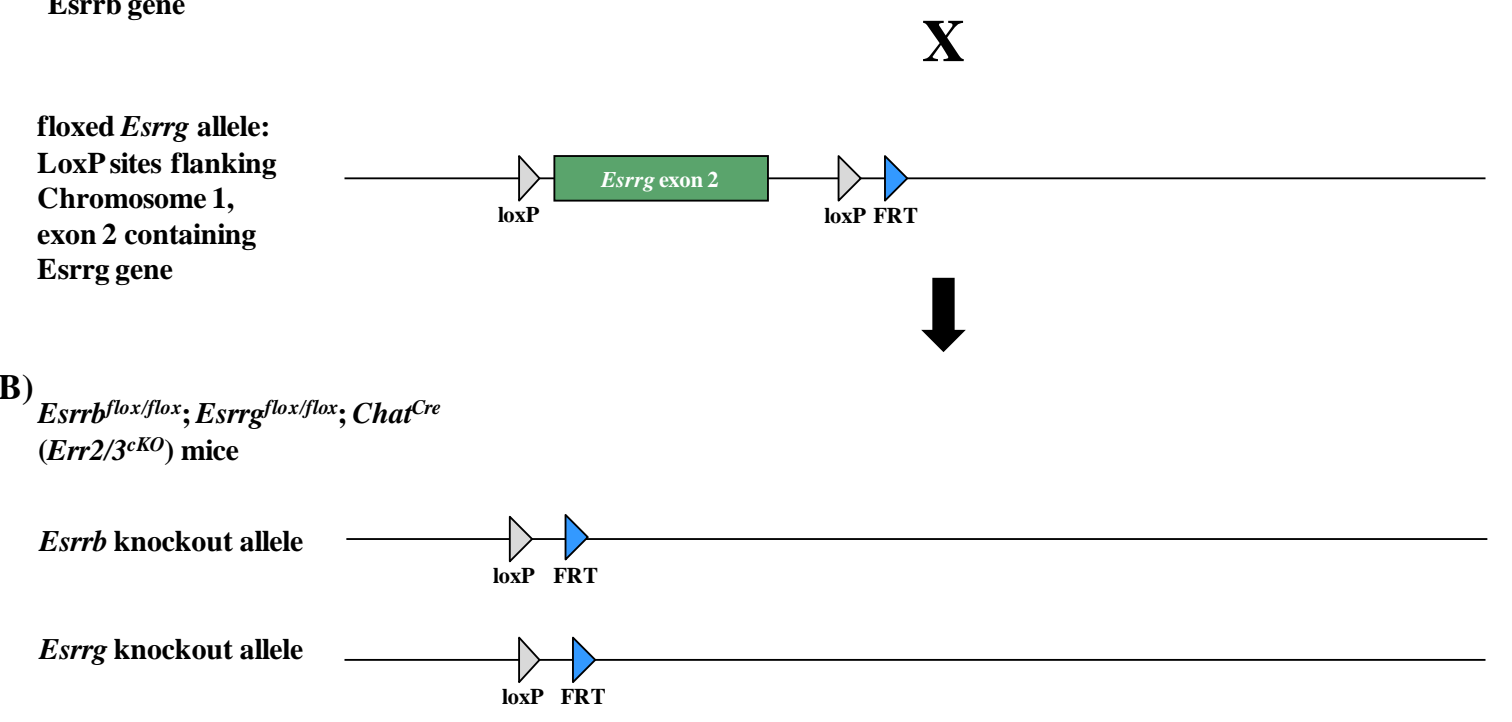

Figure 2.3 The interbreeding strategy for generating $\mathrm{Esrrb}^{\text {floxflox}}$; $\mathrm{Esrrg}^{\text {flox/flox}}$; Chat ${ }^{\mathrm{Cre}}$ conditional knockout $\left(E r r 2 / 3^{c K O}\right)$ mice lacking Err2 and Err3 in MNs

A) $C h a t^{C r e}$ mice with floxed Esrrb allele (including the transcriptional start site and open reading frame) were interbred with floxed Esrrg allele Err2/3 ${ }^{c K O}$ mice. For floxed Esrrb and Esrrg alleles, a loxP site upstream of the targeted exon 2 was inserted. Moreover, there was an insertion of loxP and FRT sites flanking the neo ${ }^{r}$ gene (Neomycin phosphotransferase) cassette downstream of the targeted exon 2. The neo ${ }^{\mathrm{r}}$ antibiotic selection marker cassette was inserted through homologous recombination technique and excised by interbreeding these mice with mice that express Flp recombinase, thus, leaving an FRT site. B) Esrrb $f^{f l o x f f l o x}$; Esrrg ${ }^{\text {floxfflox}}$; Chat ${ }^{\text {Cre }}\left(E r r 2 / 3^{c K O}\right)$ mice have deleted Esrrb and Esrrg in the MNs. (Schematics modified after Chen and Nathans, 2007; Institut Clinique de la Souris (ICS), Alsace, France).

\subsubsection{Mouse genotyping}

The mouse line genotypes were confirmed for the subsequent experiments. Genomic deoxyribonucleic acid (DNA) was extracted by incubating mouse tail biopsies in $500 \mu 1$ of lysis buffer overnight (O.N.) at $56^{\circ} \mathrm{C}$. The solution was then vortexed and centrifuged for 5 min at 14,500 r.p.m to obtain clean cell lysate. The supernatant was incubated with $500 \mu 1$ of Phenol-Chloroform, vortexed and centrifuged for $10 \mathrm{~min}$ at 14,500 r.p.m. The top phase was then collected and incubated with $500 \mu \mathrm{l}$ of Chloroform, vortexed and centrifuged for $10 \mathrm{~min}$ at 14,500 r.p.m. The top phase was collected and DNA was precipitated in $500 \mu 1$ of Isopropanol. The pellet containing the DNA was washed with $70 \%$ ethanol, vortexed and 
centrifuged at 14,500 r.p.m. and dried at $37^{\circ} \mathrm{C}$ on the heating block. The DNA was eluted in $50 \mu \mathrm{l}$ of double-distilled water $\left(\mathrm{ddH}_{2} \mathrm{O}\right)$ and stored at $-20^{\circ} \mathrm{C}$.

\subsection{Genotyping Chat::Tomato mouse line}

The polymerase chain reaction (PCR) reaction mix for the Chat-Cre mouse line contained several reagents and concentrations:

1. $2.5 \mu 1$ of Green Taq Reaction Buffer

2. $1 \mu \mathrm{l}$ of mouse tail DNA

3. $0.5 \mu 1$ of $10 \mathrm{mM}$ of dNTP mix

4. $0.5 \mu 1$ of $10 \mathrm{mM}$ of wild-type (W.T.) ChAT forward primer (GTT TGC AGA AGC GGT GGG) or mutant ChAT forward primer (CCT TCT ATC GCC TTC TTG ACG)

5. $0.5 \mu \mathrm{l}$ of $10 \mathrm{mM}$ of W.T. or mutant ChAT reverse primer (AGA TAG ATA ATG AGA GGC TC)

6. $0.5 \mu 1$ Taq DNA Polymerase

The final volume of the PCR reaction was adjusted to $25 \mu$ with $\mathrm{ddH}_{2} \mathrm{O}$.

The PCR cycle had specific parameters for this reaction:

\section{Initial Denaturation}

Temp. Time Cycles

$94^{\circ} \mathrm{C} \quad 3 \min \quad 1$

\section{Denaturation, Annealing, Elongation}

Temp. Time Cycles

$94^{\circ} \mathrm{C} \quad 30 \mathrm{sec}$.

$55^{\circ} \mathrm{C} \quad 30 \mathrm{sec} . \quad 35$

$72^{\circ} \mathrm{C} \quad 30 \mathrm{sec}$.

\section{Final Elongation}

Temp. Time Cycles

$72^{\circ} \mathrm{C} \quad 2 \min \quad 1$

Following the PCR reaction, the DNA bands were resolved using agarose the gel electrophoresis technique. An agarose gel was molded from a solution of $0.05 \mu 1 / \mathrm{ml}$ of ethidium bromide dissolved in $1 \%(\mathrm{w} / \mathrm{v})$ agarose containing Tris-acetate-EDTA (TAE) buffer solution. In order to resolve DNA bands, the DNA and loading dye were combined and filled into the solidified agarose gel lanes, placed in the electrophoresis chamber containing TAE buffer solution and exposed to a current. The gel electrophoresis DNA separation resulted in 1) one DNA band at 350 b.p. for the mutant mice, 2) two faint DNA bands at 200 b.p. and 
600 b.p. and one mutant band at 350 b.p. in homozygous $\mathrm{Cre}^{+} / \mathrm{Cre}^{+}$mice, 3) one DNA band 300 b.p. for $\mathrm{Cre}^{-}$mice, 4) one DNA band at 300 b.p. and one DNA band at 350 b.p. for $\mathrm{Cre}^{+} / \mathrm{Cre}^{-}$heterozygous mice.

The polymerase chain reaction (PCR) reaction mix for the Chat::Tomato mouse line contained several reagents and concentrations:

1. $2.5 \mu 1$ of Green Taq Reaction Buffer

2. $1 \mu \mathrm{l}$ of mouse tail DNA

3. $0.5 \mu \mathrm{l}$ of $10 \mathrm{mM}$ of dNTP mix

4. $0.5 \mu \mathrm{l}$ of $10 \mathrm{mM}$ of W.T. Rosa26-tdTomato forward primer (AAG GGA GCT GCA GTG GAG TA) or Mutant Rosa26-tdTomato forward primer (CTG TTC CTG TAC GGC ATG G)

5. $0.5 \mu \mathrm{l}$ of $10 \mathrm{mM}$ of W.T. Rosa26-tdTomato reverse primer (CCG AAA ATC TGT GGG AAG TC) or Mutant Rosa26-tdTomato reverse primer (GGC ATT AAA GCA GCG TAT CC)

6. $0.5 \mu 1$ Taq DNA Polymerase

The final volume of the PCR reaction was adjusted to $25 \mu 1$ with $d_{d d} \mathrm{H}_{2} \mathrm{O}$.

The PCR cycle had specific parameters for this reaction:

\section{Initial Denaturation}

Temp. Time Cycles

$94^{\circ} \mathrm{C} \quad 3 \min \quad 1$

\section{Denaturation, Annealing, Elongation}

Temp. Time Cycles

$94^{\circ} \mathrm{C} \quad 30 \mathrm{sec}$.

$61^{\circ} \mathrm{C} \quad 30$ sec. $\quad 35$

$72^{\circ} \mathrm{C} \quad 30 \mathrm{sec}$.

\section{Final Elongation}

Temp. Time Cycles

$72^{\circ} \mathrm{C} \quad 2 \mathrm{~min} \quad 1$

The PCR reaction and subsequent gel electrophoresis resulted in 1) one DNA band at 196 b.p. band for mutant mice, 2) two DNA bands at 297 b.p. and 196 b.p. for heterozygous mice, and 3) one DNA band at 297 b.p. for wild-type mice. 


\subsection{Genotyping of Esrrb-Chat-Cre knockout mouse line}

The polymerase chain reaction (PCR) reaction mix for the Esrrb-Chat-Cre mouse line contained several reagents and concentrations:

1. $2.5 \mu 1$ of Green Taq Reaction Buffer

2. $1 \mu 1$ of mouse tail DNA

3. $0.5 \mu 1$ of $10 \mathrm{mM}$ of dNTP mix

4. $0.5 \mu 1$ of $10 \mathrm{mM}$ of $E s r r b$ forward primer (GGG GGC CTT GGT CTA CAG GTC TAG T)

5. $0.5 \mu 1$ of $10 \mathrm{mM}$ of Esrrb reverse primer (TCT TTC TAC GGC GTT TCA GGG ACC)

6. $0.5 \mu 1$ Taq DNA Polymerase

The final volume for the PCR reaction was adjusted to $25 \mu$ l with $\mathrm{ddH}_{2} \mathrm{O}$.

The PCR cycle had specific parameters for this reaction:

\section{Initial Denaturation}

Temp. Time Cycles

$94^{\circ} \mathrm{C} \quad 3 \min \quad 1$

\section{Denaturation, Annealing, Elongation}

Temp. Time Cycles

$94^{\circ} \mathrm{C} \quad 30 \mathrm{sec}$.

$55^{\circ} \mathrm{C} \quad 30 \mathrm{sec} . \quad 35$

$72^{\circ} \mathrm{C} \quad 30 \mathrm{sec}$.

\section{Final Elongation}

Temp. Time Cycles

$72^{\circ} \mathrm{C} \quad 2 \min \quad 1$

The PCR reaction and subsequent gel electrophoresis separation resulted in 1) one DNA band at 300 b.p. for knockout mice, 2) one DNA band at 150 b.p. for W.T. mice and 3) both bands for heterozygous mice.

\subsubsection{Genotyping of Esrrg-Chat-Cre knockout mouse line}

The polymerase chain reaction (PCR) reaction mix for the Esrrg-Chat-Cre mouse line contained several reagents and concentrations:

1. $2.5 \mu 1$ of Green Taq Reaction Buffer

2. $1 \mu 1$ of mouse tail DNA

3. $0.5 \mu \mathrm{l}$ of $10 \mathrm{mM}$ of dNTP mix 
4. $0.5 \mu \mathrm{l}$ of $10 \mathrm{mM}$ of Esrrg forward primer (GTT TTA AAG GCC CTT GGT GAT CTC GC)

5. $0.5 \mu \mathrm{l}$ of $10 \mathrm{mM}$ of Esrrg reverse primer (CTG CAA CCC TTG GAC TGC CAG AAC)

6. $0.5 \mu 1$ Taq DNA Polymerase

The final volume was adjusted to $25 \mu \mathrm{l}$ with $\mathrm{ddH}_{2} \mathrm{O}$.

The PCR cycle had specific parameters for this reaction:

\section{$\underline{\text { Initial Denaturation }}$}

Temp. Time Cycles

$94^{\circ} \mathrm{C} \quad 2 \min \quad 1$

\section{Denaturation, Annealing, Elongation}

Temp. Time Cycles

$94^{\circ} \mathrm{C} \quad 1 \mathrm{~min}$

$62^{\circ} \mathrm{C} \quad 1 \min \quad 2$

$72^{\circ} \mathrm{C} \quad 1 \mathrm{~min}$

\section{Denaturation, Annealing, Elongation}

Temp. Time Cycles

$94^{\circ} \mathrm{C} \quad 30 \mathrm{sec}$.

$62^{\circ} \mathrm{C} \quad 30 \mathrm{sec} . \quad 30$

$72^{\circ} \mathrm{C} \quad 30 \mathrm{sec}$.

\section{Final Elongation}

Temp. Time Cycles

$72^{\circ} \mathrm{C} \quad 3 \min \quad 1$

The PCR reaction and subsequent gel electrophoresis for DNA separation resulted in 1) one DNA band at 300 b.p. for knockout mice, 2) one DNA band at 150 b.p. for W.T. mice, and 3) both DNA bands for heterozygous mice.

\subsection{Mouse behavior experiments}

\subsubsection{The Digigait ${ }^{\mathrm{TM}}$ treadmill task}

Mouse gait was analyzed using the DigiGait ${ }^{\mathrm{TM}}$ Imaging System (Mouse Specifics Inc., Framingham, MA U.S.A.) (Amende et al., 2005; Yuengert et al., 2015). The DigiGait ${ }^{\mathrm{TM}}$ Imaging System uses a motorized treadmill equipped with a transparent belt and a high-speed digital video camera placed from below to capture animal locomotion. The videos were 
acquired using DigiGait ${ }^{\mathrm{TM}}$ Imager software and processed using DigiGait ${ }^{\mathrm{TM}}$ Analysis software (version 14.0).

$C h A T^{\text {Cre }}$ (control, N=9) and Err2 $2^{\text {floxfflox }} ; \operatorname{Err} 3^{\text {floxfflox }} ; \operatorname{ChAT}^{\text {Cre }}\left(E r r 2 / 3^{c K O}, \mathrm{~N}=8\right)$ at 8 weeks were placed into the treadmill chamber to acclimatize to the treadmill for $5 \mathrm{~min}$. After acclimatization, the mice were recorded with DigiGait ${ }^{\mathrm{TM}}$ Imager software for sequential trials at 5 different belt speeds $(10 \mathrm{~cm} / \mathrm{s}, 15 \mathrm{~cm} / \mathrm{s}, 20 \mathrm{~cm} / \mathrm{s}, 25 \mathrm{~cm} / \mathrm{s}, 30 \mathrm{~cm} / \mathrm{s}, 35 \mathrm{~cm} / \mathrm{s})$ for 3 trials per speed. The acquired videos were processed using DigiGait ${ }^{\mathrm{TM}}$ Analysis software (version 14.0). The software records the pixel area of a digital paw print (Paw Contact Area) with respect to time (sec) or (S) and a signal trace is produced (Fig. 2.4A, B) (Mouse Specifics, 2011). Five phases of movement were captured during a step-cycle: 1) Stride, 2) Stance, 3) Swing, 4) Braking, 5) Propulsion (Fig. 2.4A). Various indices were obtained that enabled the assessment of the different locomotion dynamics and posture to compare control versus Err $2 / 3^{c K O}$ mice. 
A)

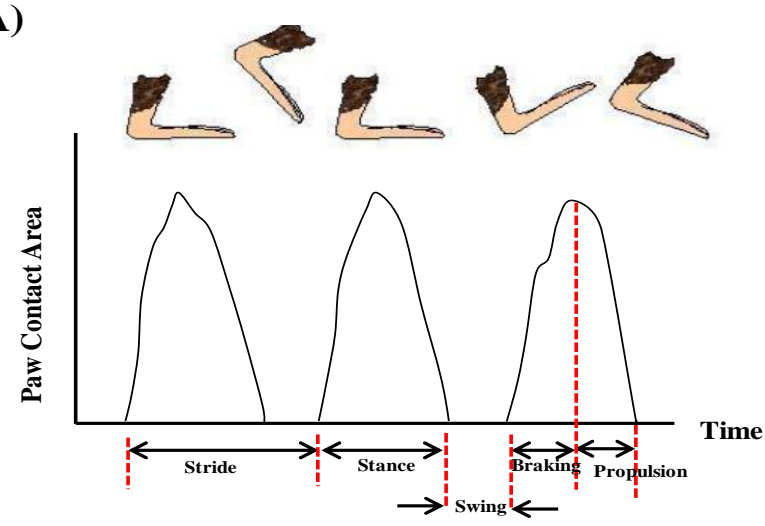

B)
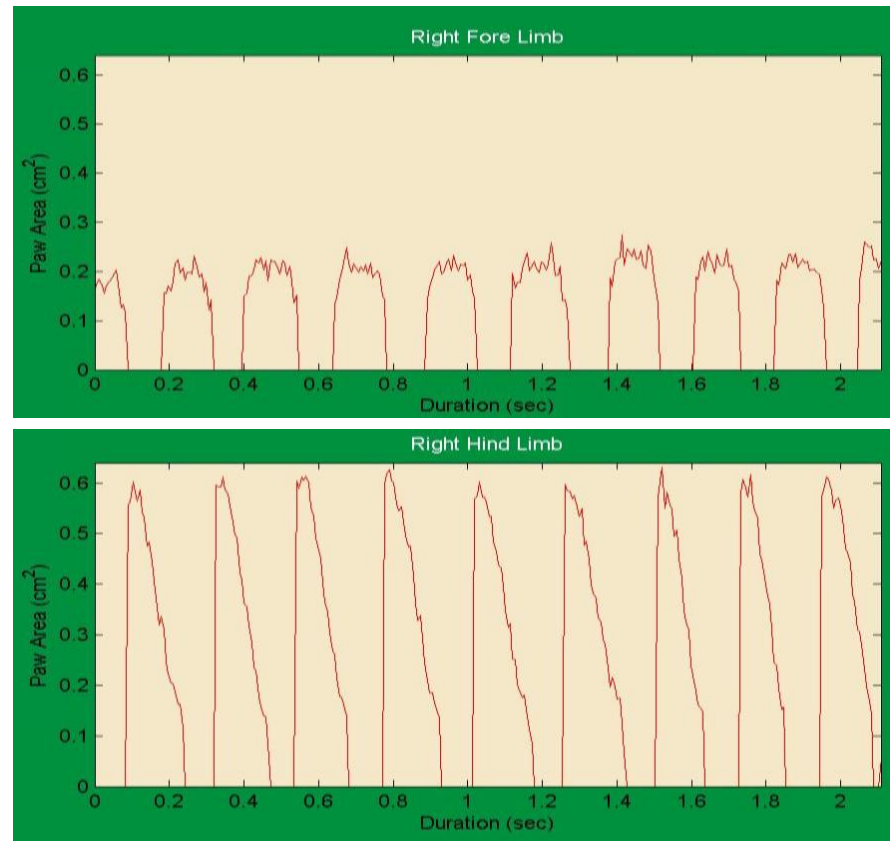

Figure 2.4 Schematic diagrams showing digital paw print (Paw Contact Area) and gait signals for the forelimbs and hindlimbs

A) Several step-cycle phases were used as signals: 1) Stride phase: the time of Stance plus Swing phases, 2) Stance phase: the time paw is touching the ground, 3) Swing phase: the time the paw is off the ground, 4) Braking phase and 5) Propulsion phase: two phases of the Stance phase that can be separated into Braking phase (coming to a stop) and Propulsion phase (starting). B) A walking mouse $(18 \mathrm{~cm} / \mathrm{S})$ generates a gait trace of distinct fore and hind paw gait signals with respect to Duration (sec) or (S) and Paw Contact Area $\left(\mathrm{cm}^{2}\right)$. (Fig. 2.4A was modified after Digigait ${ }^{\mathrm{TM}}, 2011$ )

\subsubsection{Orthogonal Signal Correction Partial Least Squares (OSC-PLS) analysis}

The Orthogonal Signal Correction Partial Least Squares (OSC-PLS) multivariate data analysis for the Digigait ${ }^{\mathrm{TM}}$ treadmill task was performed by Camille Lancelin, Ph.D. A different analysis method was used instead of the classical multivariate methods since Digigait $^{\mathrm{TM}}$ analysis generated many different gait variables compared to the low numbers of mice in control and $E r r 2 / 3^{c K O}$ groups and/or the inability to segregate data because of 
redundant variables. Given that the previous studies effectively used the Principle Component Analysis (PCA), which is similar to Partial Least Squares (PLS) and Partial Least Square Discriminate Analysis (PLS-DA) designed for sets of discrete variables to describe movement behavior in mice, therefore, the Orthogonal Signal Correction Partial Least Squares (OSC-PLS) method was used to segregate continuous variable data into predictive and uncorrelated categories (Poulet et al., 2014; Takeoka et al., 2014; Nilsson et al., 2006). The OSC-PLS allows for better analytics and interpretation of motor behavior as an elaborate method of PLS. The OSC-PLS method enlarges the explained variance between-groups in a component or dimension and the within-group variance is segregated in the orthogonal component or dimension. To analyze the motor behavior data, an R-script that was created for chemometric analysis was adjusted (R Core Team, 2015; Grapov, 2014; Wherens, 2011).

The OSC-PLS method analysis consisted of several steps. First, the raw data was standardized to construct a model. To construct a model, the data was segregated into a "training set," and to validate the model, the data was segregated into a "testing set." Since the observations or mice $(<20)$ was a low quantity, the leave-one-out (LOO) method was used to construct a model. The LOO method excludes one sample $(\mathrm{N})$, which becomes the "testing set" and the rest of the dataset becomes the "training set." The "training set" is used to construct a model, which entails revealing patterns of predictive relationships. To predict or categorize the "testing set" into a group, the regression equation that is produced from the "training set" is employed. The selected model was validated by evaluating its ability to categorize groups versus 100 different randomly permuted models and their ability to categorize groups. The cross-validated coefficient of determination $\left(\mathrm{Q}^{2}\right)$ and the Root Mean Square Error of Prediction (RMSEP) were used to evaluate the selected model's predictive power to the randomly permuted models. The model's "goodness of fit" to the "training set" was obtained by looking at the model's $\mathrm{Q}^{2}$ versus the $\mathrm{Q}^{2}$ of the randomly permuted model (Table 9.3 in Appendix 3) (Wherens, 2011). The chosen model's "goodness of prediction" on the "testing set" was obtained by observing the selected model's RMSEP versus the RMSEP of the randomly permuted (Table 9.3 in Appendix 3) (Wherens, 2011). Overall, the selected model indicates that there is a significant difference between the control and $E r r 2 / 3^{c K O}$ animal groups based on the totality of gait variables or indices. Furthermore, the best variables or predictors of the model were used from the first component (PCA-1) of the validated model and the loadings of the best predictors were ranked on their ability to segregate control versus Err $2 / 3^{c K O}$ mice. 
Table 2.9 Defined variables for forelimbs and hindlimb

\begin{tabular}{|c|c|c|}
\hline Variable Name & $\begin{array}{l}\text { Variable } \\
\text { units }\end{array}$ & Variable meaning \\
\hline SWING & (s) & $\begin{array}{l}\text { The duration of the swing } \\
\text { phase }\end{array}$ \\
\hline X.SWING.STRIDE & $(\%)$ & $\begin{array}{l}\text { The \% of the stride spent in } \\
\text { swing }\end{array}$ \\
\hline BRAKE & (s) & $\begin{array}{l}\text { The duration of the braking } \\
\text { phase }\end{array}$ \\
\hline X.BRAKE.STRIDE & $(\%)$ & $\begin{array}{l}\text { The } \% \text { of the stride spent in } \\
\text { braking }\end{array}$ \\
\hline PROPEL & (s) & $\begin{array}{l}\text { The duration of the propulsion } \\
\text { phase }\end{array}$ \\
\hline X.PROPEL.STRIDE & $(\%)$ & $\begin{array}{l}\text { The } \% \text { of the stride spent in } \\
\text { propulsion }\end{array}$ \\
\hline STANCE & (s) & $\begin{array}{l}\text { The duration of the stance } \\
\text { phase }\end{array}$ \\
\hline X.STANCE.STRIDE & $(\%)$ & $\begin{array}{l}\text { The } \% \text { of the stride spent in } \\
\text { stance }\end{array}$ \\
\hline STRIDE & (s) & The duration of the stride \\
\hline X.BRAKE.STANCE & $(\%)$ & $\begin{array}{l}\text { The } \% \text { of the stance phase spent } \\
\text { in braking }\end{array}$ \\
\hline X.PROPEL.STANCE & $(\%)$ & $\begin{array}{l}\text { The } \% \text { of the stance phase spent } \\
\text { in propulsion }\end{array}$ \\
\hline STANCE.SWING & (real\#) & The stance to swing ratio \\
\hline STRIDE.LENGTH & $(\mathrm{cm})$ & The length of the stride \\
\hline STRIDE.FREQUENCY & (steps/s) & The frequency of strides \\
\hline PAW.ANGLE & (deg) & The paw angle \\
\hline ABS.PAW.ANGLE & (deg) & The absolute paw angle \\
\hline PAW.ANGLE.VAR & (deg) & $\begin{array}{l}\text { The step-to-step variability in } \\
\text { paw angle }\end{array}$ \\
\hline STANCE.WIDTH & $(\mathrm{cm})$ & The width of the stance \\
\hline STEP.ANGLE & (deg) & The step angle \\
\hline STRIDE.LENGTH.VAR & $(\mathrm{cm})$ & $\begin{array}{l}\text { The step-to-step variability in } \\
\text { stride length }\end{array}$ \\
\hline STANCE.WIDTH.VAR & $(\mathrm{cm})$ & $\begin{array}{l}\text { The step-to-step variability in } \\
\text { stance width }\end{array}$ \\
\hline STEP.ANGLE.VAR & (deg) & $\begin{array}{l}\text { The step-to-step variability in } \\
\text { step angle }\end{array}$ \\
\hline STRIDE.LENGTH.CV & $(\mathrm{CV} \%)$ & $\begin{array}{l}\text { The coefficient of variation of } \\
\text { stride length }\end{array}$ \\
\hline STANCE.WIDTH.CV & $(\mathrm{CV} \%)$ & $\begin{array}{l}\text { The coefficient of variation of } \\
\text { stance width }\end{array}$ \\
\hline STEP.ANGLE.CV & $(\mathrm{CV} \%)$ & $\begin{array}{l}\text { The coefficient of variation of } \\
\text { step angle }\end{array}$ \\
\hline SWING.DURATION.CV & $(\mathrm{CV} \%)$ & $\begin{array}{l}\text { The step-to-step variability in } \\
\text { swing duration }\end{array}$ \\
\hline PAW.AREA & $\left(\mathrm{cm}^{\wedge} 2\right)$ & The area of the paw \\
\hline PAW.AREA.VAR & $\left(\mathrm{cm}^{\wedge} 2\right)$ & $\begin{array}{l}\text { The step-to-step variability in } \\
\text { paw area }\end{array}$ \\
\hline STANCE.FACTOR & (real\#) & The stance factor \\
\hline GAIT.SYMMETRY & (real\#) & The symmetry of the gait \\
\hline MAX.DA.DT & $\left(\mathrm{cm}^{\wedge} 2 / \mathrm{s}\right)$ & $\begin{array}{l}\text { The maximal rate of change of } \\
\text { paw area during the braking } \\
\text { phase }\end{array}$ \\
\hline
\end{tabular}




\begin{tabular}{|c|c|c|}
\hline MINDA.DT & $\left(\mathrm{cm}^{\wedge} 2 / \mathrm{s}\right)$ & $\begin{array}{l}\text { The maximal rate of change of } \\
\text { paw area during the propulsion } \\
\text { phase }\end{array}$ \\
\hline OVERLAP.DISTANCE & $(\mathrm{cm})$ & The distance of overlap \\
\hline PPP & $(\mathrm{cm})$ & The paw placement positioning \\
\hline ATAXIA.COEFF & (real\#) & The coefficient of ataxia \\
\hline MIDLINE.DISTANCE & $(\mathrm{cm})$ & The distance to the midline \\
\hline FORELIMB.WEIGHT.SUPPORT & (real\#) & The forelimb weight support \\
\hline ABS.AXIS.DISTANCE & $(\mathrm{cm})$ & The absolute distance to the axis \\
\hline NORM.STRIDE.LENGTH & (real\#) & The normalized stride length \\
\hline NORM.STANCE.WIDTH & (real\#) & The normalized stance width \\
\hline SHARED.STANCE (Hindlimbs only) & (s) & $\begin{array}{l}\text { The dual stance or double } \\
\text { support }\end{array}$ \\
\hline X.SHARED.STANCE (Hindlimbs only) & $(\%)$ & $\begin{array}{l}\text { The } \% \text { dual stance or double } \\
\text { support }\end{array}$ \\
\hline TAU.PROPULSION (Hindlimbs only) & (real\#) & The propulsion time constant \\
\hline PAW.DRAG (Hindlimbs only) & $\left(\mathrm{mm}^{\wedge} 2\right)$ & The paw drag \\
\hline PAW.LENGTH (Hindlimbs only) & $(\mathrm{cm})$ & The length of the paw \\
\hline PAW.LENGTH.VAR (Hindlimbs only) & $(\mathrm{cm})$ & $\begin{array}{l}\text { The variability in the length of } \\
\text { the paw }\end{array}$ \\
\hline PAW.WIDTH (Hindlimbs only) & $(\mathrm{cm})$ & The width of the paw \\
\hline PAW.WIDTH.VAR (Hindlimbs only) & $(\mathrm{cm})$ & $\begin{array}{l}\text { The variability in the width of } \\
\text { the paw }\end{array}$ \\
\hline SFI (Hindlimbs only) & $(\mathrm{cm})$ & The sciatic function index \\
\hline PFI (Hindlimbs only) & $(\mathrm{cm})$ & The peroneal function index \\
\hline TFI (Hindlimbs only) & $(\mathrm{cm})$ & The tibial function index \\
\hline
\end{tabular}

\subsubsection{The horizontal ladder task}

Fine motor skills of mice were recorded using a sliding track installed with a camera to view the animal from the bottom while it walked across the horizontal ladder with modified specifications: animal ladder was $100 \mathrm{~cm}$ in length, rung diameter of $3 \mathrm{~mm}$ and rung spaced at $7 \mathrm{~mm}$ (mice tested at alternating rungs spaced at $14 \mathrm{~mm}$ ) (Akay et al., 2014). $C h A T^{C r e}$ (control, N=5, females) and Err2 $2^{\text {floxflox }} ; \operatorname{Err}^{\text {floxfllox }^{\text {f }} \text { ChAT }}{ }^{\text {Cre }}\left(E r r 2 / 3^{\text {cKO }}, \mathrm{N}=5\right.$, females) age-matched at 8 weeks were trained to go to their home cage located at the ladder's end and rested for 1 min between trials. The mice were trained for 2 days ( 3 trials per animal, single direction) the first week and then trained 2 days ( 3 trials per animal, single direction) the second week and tested on the third day (4-5 trials per animal, single direction towards home cage).

The videos were captured and processed using the same protocol for control and Err $2 / 3^{c K O}$ mice. Mouse locomotion was acquired using GoPro HD Hero2 (GoPro Inc., U.S.A) at 120 f.p.s., at 848x480 image size and stored as MP4 files for analysis. For the figure videos and images, GoPro Studio Version (2.5.4) and proDAD Defishr Version 1.0 (proDAD $\mathrm{GmbH}$, Immendingen, Germany) were used to decrease the speed to $25 \%$ of the original speed, decrease the frames to 60 f.p.s. and eliminate the fish-eye effect by applying Mobius A Wide presets and zoom modified to 110.0 for final videos and images. 
The mouse hindlimbs were pooled for data quantification. A "hit" or correct placement was scored when the hind paw was placed with stability in the middle of the rung. A "miss" was scored when the hind paw slipped through missing the rung (complete miss) or when the hind paw failed to locate the rung (correction miss). For each control and Err $2 / 3^{c K O}$ mouse, on average, about 40 steps per trial were quantified.

A "Miss Index" was used for quantification. To compare control and $E r r 2 / 3^{c K O}$ mice, $\%$ misses was calculated: \# of misses/total \# of steps (hits + misses) was calculated for control mice. This number was then normalized by taking its inverse to give a multiplication factor to compare with $\operatorname{Err} 2 / 3^{c K O}$ mice. The multiplication factor was then multiplied by $\%$ misses of Err $2 / 3^{c K O}$ mice. This resulted in a factor or "Miss Index," which is a normalized value to compare the tendency to misses between control and $\operatorname{Err} 2 / 3^{c K O}$ mice.

\subsubsection{The horizontal beam task}

An additional task for analysis of fine motor skills was performed for older mice. The task was recorded using a sliding track installed with a camera on the side to view the animal while it walked across a horizontal beam with modified specifications: $20 \mathrm{~mm}, 25 \mathrm{~mm}$ or 30 $\mathrm{mm}$ in width and $100 \mathrm{~cm}$ in length (Bourane et al., 2015). $C h A T^{\text {Cre }}$ (control, $\mathrm{N}=4$, females) and $E r r 2^{\text {floxflox }}$; Err $3^{\text {floxflox }}$; $C h A T^{C r e}\left(E r r 2 / 3^{c K O}, \mathrm{~N}=4\right.$, females) age-matched $\sim 10$ months-old mice were trained to enter into a home cage located at the beam's end and rest for $1 \mathrm{~min}$ between trials in the home cage. For the experiment, mice were trained for 3 days ( 4 trials per animal, forward and backward on beam) and subsequently, tested the next day for the same amount of trials.

Similar to the horizontal ladder task, the videos were captured and processed using the same protocol for control and $E r r 2 / 3^{c K O}$ mice. The final videos and images for the figures were slowed to $40 \%$ of original speed and reduced to 60 f.p.s. using the GoPro Studio Version (2.5.4) and the zoom was adjusted to 180.0 using proDAD Defishr Version 1.0 (proDAD GmbH, Immendingen, Germany).

Similar to the horizontal ladder task, the animal hindlimbs were pooled for data quantification. A "hit" or correct placement was scored when the hind paw was placed flat on the beam or when the hind paw straddled the side of the beam with a grip. A "miss" was scored the hind paw slips/hangs on the side of the beam without grip (complete miss) or when the mouse failed to locate the beam (correction miss). For control and Err2/3 ${ }^{c K O}$ mice, an average of $\sim 9-16$ steps per trial was quantified. Quantification was carried out similar to the "Miss Index" used for the horizontal ladder task. 


\subsection{Quantitative immunodetection studies}

\subsubsection{Chick tissue processing}

The spinal cord thoracolumbar region (T10-L5) from E5.5-E6.5 (HH St. 27-30) chick embryos were dissected and incubated in $4 \%$ paraformaldehyde (PFA) fixative solution for 2 $\mathrm{h}$ at $4^{\circ} \mathrm{C}$. The tissue was then incubated in PBS for $24 \mathrm{~h}$ and then in a solution containing $30 \%$ sucrose in PBS for $5 \mathrm{~h}$. The tissue was embedded in a mold with O.C.T. cryoprotectant (Sakura Finetek USA, Inc.) and stored at $-80^{\circ} \mathrm{C}$. The tissue was sectioned using Leica CM1850 UV cryostat (Leica Microsystems $\mathrm{GmbH}$ ) into $30 \mu \mathrm{m}$ thick slices and collected on microscope slides (Superfrost ${ }^{R}$ Plus, Thermo Fisher Scientific) and allowed to dry at room temperature (RT) $\left(22^{\circ} \mathrm{C}\right)$ and stored at $-20^{\circ} \mathrm{C}$.

\subsubsection{Chick quantitative immunodetection studies}

The cryosections were washed with PBS ( 3 washes $x 5 \mathrm{~min} /$ wash) and treated with appropriate concentrations primary antibodies (Table 2.1) diluted in a solution of PBS containing $1 \%$ BSA and $0.5 \%$ Triton-X 100. The slides were incubated with primary antibodies overnight (O.N.) at $4^{\circ} \mathrm{C}$. These slides were then washed with PBS (3 washes x 5 $\mathrm{min} / \mathrm{wash}$ ) and incubated in appropriate concentrations of secondary antibodies for $1 \mathrm{~h}$ at RT $\left(22^{\circ} \mathrm{C}\right.$ ) (Table 2.2). The slides were then washed with PBS ( 3 washes $x 5 \mathrm{~min} / \mathrm{wash}$ ) and mounted using 50\% glycerol in PBS and applied with coverslips (Carl Roth $\mathrm{GmbH}+\mathrm{Co}$. $\mathrm{KG})$.

\subsubsection{Fluoro-Gold (FG) MN labeling experiments}

Previous studies have successfully used Fluoro-Gold (FG) (Fluorochrome LLC., Denver, CO.) for labeling MNs (Tosolini et al., 2013). FG is comprised of a chemical called Hydroxstilbamadine, which is a weak base that may become incorporated in lysosomes and endosomes through a $\mathrm{pH}$ gradient (Wessendorf, 1991). In spinal MNs, the incorporation of FG occurs through endocytosis of AMPA ( $\alpha$-Amino-3-hydroxy-5-methyl-4isoxazolepropionic acid) receptors (Falgairolle and O'Donovan, 2015). For retrograde labeling of MNs, mice were intraperitoneally injected with $0.5 \%$ (w/v) FG dissolved in PBS $(\mathrm{pH}=7.2)$ and volume $0.10 \mathrm{ml} / 5 \mathrm{~g}$ body weight was administered. Mice were sacrificed 1-day (electrophysiology studies) or 4-days (quantitative immunodetection studies) post-FG injection. 


\subsubsection{Mouse tissue processing}

Anesthetized mice were transcardially perfused with $25 \mathrm{ml}$ of cold PBS ( $\mathrm{pH}=7.2)$ and subsequently, with $25 \mathrm{ml}$ of cold $4 \%$ paraformaldehyde (PFA) fixative solution 4-days postFG tracer injection. The spinal cord thoracolumbar region (T10-L5) and brain tissue were dissected and post-fixed for $3 \mathrm{~h}$ in $4 \% \mathrm{PFA}$ at $4{ }^{\circ} \mathrm{C}$ and subsequently, $1 \%$ PFA overnight (O.N.) at $4^{\circ} \mathrm{C}$. The tissue was then incubated in PBS $(\mathrm{pH}=7.2)$ for about $36 \mathrm{~h}$ at $4{ }^{\circ} \mathrm{C}$ and then in $30 \%$ sucrose in PBS O.N. at $4^{\circ}$ C. The tissue was embedded with O.C.T. cryoprotectant (Sakura Finetek USA, Inc.) in a mold and stored at $-80^{\circ} \mathrm{C}$. The tissue was sectioned using Leica CM1850 UV cryostat (Leica Microsystems $\mathrm{GmbH}$ ) into $30 \mu \mathrm{m}$ thick slices (mouse spinal cord tissue) or $50 \mu \mathrm{m}$ thick slices (mouse midbrain tissue) and collected on microscope slides (Superfrost ${ }^{R}$ Plus, Thermo Fisher Scientific) and allowed to dry at RT $\left(22^{\circ} \mathrm{C}\right)$ and stored at $-20^{\circ} \mathrm{C}$.

\subsubsection{Mouse quantitative immunodetection studies}

The cryosections were washed with PBS ( 3 washes x $5 \mathrm{~min} /$ wash) and treated with appropriate concentrations primary antibodies (Table 2.1 in section 2.1) diluted in PBS containing $1 \%$ BSA and $0.5 \%$ Triton-X 100. The slides were incubated with primary antibodies overnight (O.N.) at $4^{\circ} \mathrm{C}$ or for $4 \mathrm{~h}$ at RT $\left(22^{\circ} \mathrm{C}\right)$. Slides were then washed with PBS (3 washes x $5 \mathrm{~min} /$ wash) and incubated in appropriate concentrations of secondary antibodies for $1 \mathrm{~h}$ at $\mathrm{RT}\left(22^{\circ} \mathrm{C}\right)$ (Table 2.2 in section 2.2). The slides were then washed with PBS (3 washes x 5 min/wash) and mounted using 50\% glycerol in PBS and applied with coverslips (Carl Roth GmbH + Co. KG).

\subsubsection{Chick and mouse confocal imaging and quantification}

After incubating cryosections with the appropriate concentrations of primary and secondary antibodies, they were imaged using Zeiss LSM 710 laser scanning microscope and Zeiss LSM 800 microscopes equipped with 10x, 20x, and 40x (oil immersion objective) objectives. Photomultiplier (PMT) settings were applied carefully to avoid any spectral bleedthrough across channels. With respect to the cryosection thickness, 6-28 optical sections (for mouse studies) and 10-64 optical sections (for chick studies) were obtained from the ventral horn of the spinal cord (for chick and and mouse studies) and the oculomotor nucleus of the midbrain (for mouse studies) at a step-size of 0.8-1.50 $\mu \mathrm{m}$. .CZI files were imported into ImageJ software and applied the "Z-project" function at max intensity. The raw pixel signal was quantified as fluorescence intensity or expression levels by using the Lasso Tool to 
delineate the MN nuclei in Adobe Photoshop CS5.1. The same procedure was used to quantify all immunodetection experiments: the fluorescence intensity was normalized to the neuron with the highest fluorescent intensity of expression after normalizing for non-specific background typically observed in cryosections during immunodetection experiments. Zprojected images were processed using "levels" function to adjust brightness and contrast uniformly across the section and were incorporated into the figure panel. Figure panels were organized using Adobe Illustrator CS5.1.

\subsubsection{Quantification of vGlut1 synaptic varicosities using Imaris software}

Transversal cryosections were treated with the appropriate concentration of antibodies (Table 2.1 and Table 2.2) and imaged using 40x (oil immersion objective) objective equipped on Zeiss LSM 710 laser scanning microscope. A step-size of $0.80 \mu \mathrm{m}$ was used to obtain 820 optical sections to obtain Raw Z-stack Carl Zeiss files (.czi). Using Imaris 8.0 software (Bitplane AG, Switzerland), neural surfaces were rendered using the "Create Surface" function and spots representing vGlut1 synaptic varicosities were created to identify them using the "Create Spots" function. Using the "find spots close to surface" function, vGlut1 synaptic varicosities were defined and identified on the soma and proximal dendritic membrane of MNs labeled with Fluoro-Gold (FG). A distance threshold of $2 \mu \mathrm{m}$ from the neuron surface (within established values (0.7-2.5 $\mu \mathrm{m}$ ) of synaptic morphology from previous studies) to quantify the number of vGlut1 synaptic varicosities apposed onto MNs (Fogarty et al., 2013).

\subsection{In ovo electroporation experiments}

Lohmann LSL strain fertilized chicken eggs were incubated at $37.8^{\circ} \mathrm{C}$ and $80 \%$ humidity until E2.7-3.0 (HH St. 14-18). The embryos were then extracted $5 \mathrm{ml}$ of their albumin using a syringe and partially removed of their eggshell to expose the embryo. The DNA constructs were injected into the central canal of the neural tube. In the forced expression experiments, the final concentration of DNA constructs was $\sim 1.5 \mu \mathrm{g} / \mu \mathrm{l}$. The molar ratio of the DNA expression constructs, transposase DNA construct, fast green-positive DNA dye was 2:1:1, respectively. The final concentration of DNA constructs electroporated for mouse KcnalO enhancer experiments was $1.5 \mu \mathrm{g} / \mu 1,1.5 \mu \mathrm{g} / \mu 1,1.5 \mu \mathrm{g} / \mu 1,0.3 \mu \mathrm{g} / \mu 1$ for the mouse Kcnalo enhancer (control and mutant), CMV-eGFP, CMV-VP16-Err2-eGFP, and transposase DNA construct, respectively. A specific molar ratio of 5:5:5:1:0.5 was injected for the mouse Kcnal0 enhancer (control and mutant), CMV-eGFP, CMV-VP16-Err2-eGFP, 
transposase DNA construct and fast green-positive DNA dye, respectively. The final concentration of DNA constructs were electroporated on one side (unipolar) of the neural tube using the ECM 830 electroporation system (BTX Instrument Division, Harvard Apparatus Inc.) with the parameters of 5 pulses of $25 \mathrm{mV}$ in the LV $99 \mathrm{~ms} / 500 \mathrm{~V}$ modus. The eggs were sealed with tape and incubated until harvesting.

\subsubsection{Tol2 transposon system: stable in ovo transfection of chick MNs}

The chicken embryo is a useful model organism for developmental studies in the spinal cord since it has a precocial developmental strategy where the nervous system and neuromuscular system are highly developed during in ovo gestation (Fig. 2.5). In this developmental model, gene expression can be manipulated in ovo during development. However, a major constraint of the system is that electroporated genes have transient expression due to the lack of integration into chromosomes and the dilution of the electroporated DNA construct as cells proliferate (Sato et al., 2007). To stably integrate the gene of interest into chicken chromosome, a transposon-mediated system was developed (Kawakami and Shima, 1999). To study how Err2 and Err3 function during late embryonic stages of chick, the Tol2 transposon system cloned from the Medaka fish (Oryzias latipes) genome (Kawakami and Shima, 1999; Kawakami, 2007; Sato et al., 2007). The Tol2 transposon element encodes a functional transposase, which can recognize Tol2 sequences and catalyzes the transposition of DNA inserts between two Tol2 sites (Sato et al., 2007). Two DNA constructs were co-electroporated: 1) A gene of interest is included in the DNA construct which is regulated by the $C M V$ enhancer/promoter that is flanked by two Tol2 sites and 2) helper DNA construct comprising of the transposase gene, which is regulated by the ubiquitous CAGGS promoter (chick $\beta$-actin promoter) (Fig. 2.6A) (Kawakami, 2007; Sato et al., 2007). The $C M V$ enhancer can express the transgene in MNs (Fig. 2.6B-D) that can be recorded electrophysiologically (Lee, 2013). Within the Tol2 construct, the Multiple Cloning Sites (MCS), where the gene of interest is subcloned, is inserted between $C M V$ enhancer and the 2AeGFP coding sequence. The 2A peptide's (Aphthoviruses) role is to act as a bridge or junction between the virus capsid and the replication polyprotein domains (Trichas et al., 2008). Because it has ribosomal skipping activity, the $2 \mathrm{~A}$ peptide is termed "cis-acting hydrolase element" (CHYSEL), thus, the gene of interest-2A-eGFP is translated as a fusion protein. VP16 is a polypeptide that controls the transcription of herpes simplex virus-1 (HSV1) immediate early (IE) genes through its cis-regulatory element mechanism and also has a trans-activating mechanism (Triezenberg et al., 1988). Thus, VP16 activation domain 
(amplified from pActPL-VP16AD DNA construct) (Addgene) was used to increase the activity of transcription factors by fusing it to them (Weir, 2001). Studies have shown that Engrailed (En) is a Drosophila homeodomain protein is important for the determination of cell fate in body segmentation (Heemskerk et al., 1991). To suppress the activity of transcription factors, minimal repression domain of En can be used to fuse with heterologous DNA binding domain (Han and Manley, 1993). Thus, EnR repression domain (amplified from CAG-EnR DNA construct) (Addgene) was used to suppress the activity of transcription factors.

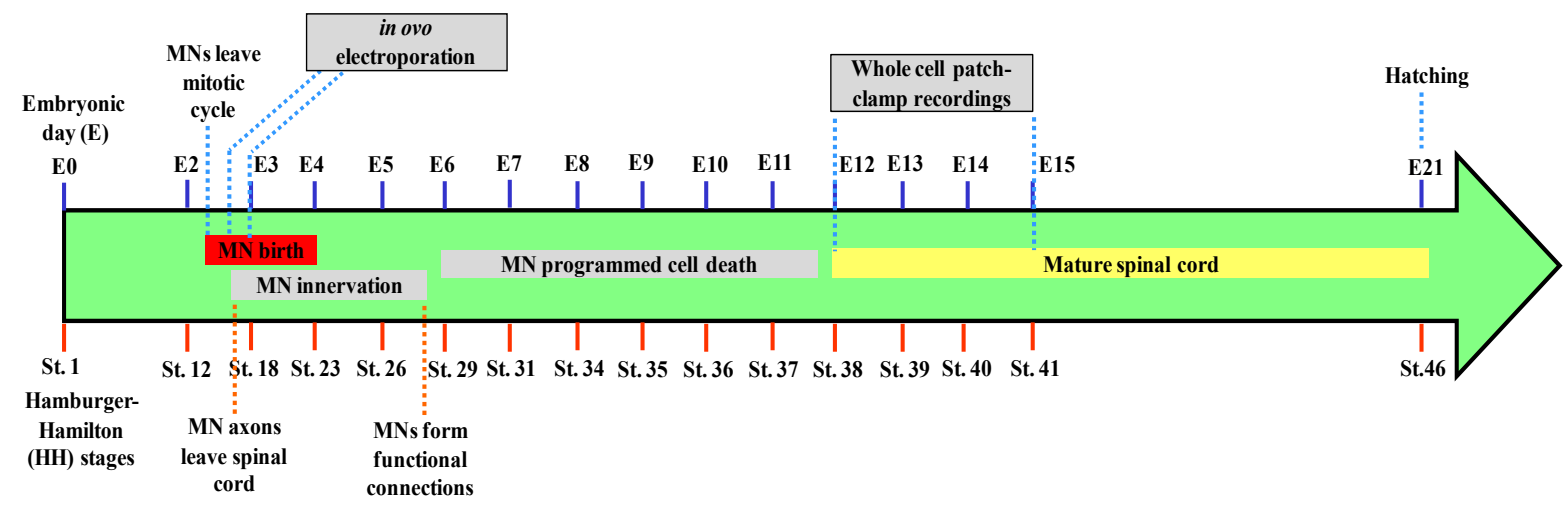

\section{Figure 2.5 MN development in chick spinal cord}

A developmental time-line of the major events in $\mathrm{MN}$ development indicated by a gradient marked by approximate embryonic days, which are correlated with Hamburger-Hamilton (HH) stages in chick. Whole cell patch-clamp recordings were carried out in MNs from relatively mature spinal cords. (Adapted from Leber et al., 1990)

Mouse Err2 and Err3 DNA constructs were cloned by Dr. Tsung-I Lee (Lee, 2013). For cloning Err2 and Err3 DNA constructs, total RNA was isolated with the Trizol reagent from E18.5 (HH St. 44.5) chick spinal cords. Mouse Err2 (NM_011934.4) and mouse Err3 (NM_011935.3) cDNA was synthesized using PrimeScript 1st cDNA Synthesis Kit (Takara Bio Inc.) following manufacturer's procedures and PCR amplified with primers:

1. Err2: Forward Primer-5'CATGCCATGGATGTCGTCCGAAGACAGGCACC' and Reverse Primer-5'CATGCCATGGCACCTTGGCCTCCAGCATCTCCAGG 3'

2. Err3: Forward Primer- 5' CATGCCATGGATGGATTCGGTAGAACTTTGC 3' and Reverse Primer- 5' CATGCCATGGGACCTTGGCCTCCAGCATTTCC 3' 
A)

\section{CMV-VP16-Err2-eGFP (Tol2 construct with gene of interest)}
pCAGGS-T2TP (transposase- expression- plasmid)
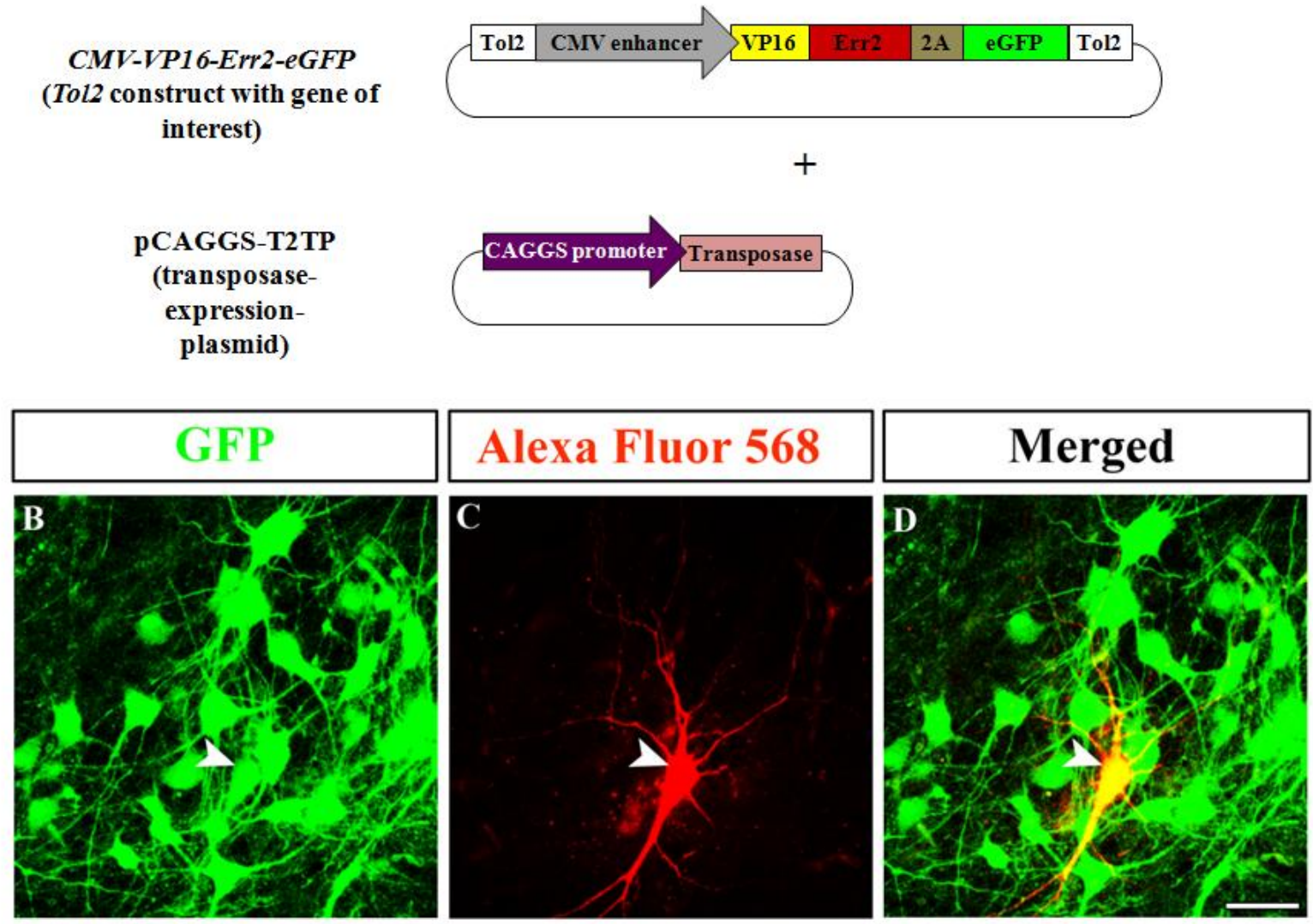

Figure 2.6 Stable transfection of chick MNs in the ventral horn of the spinal cord followed by whole cell patch-clamp recordings

A) A Tol2 construct with two Tol2 sites flanking CMV enhancer and VP16-Err2-eGFP (gene of interest) is co-electroporated with chick $\beta$-actin promoter driving the expression of transposase to express transgene in ventral horn, including MNs (Adapted from Sato et al., 2007; Lee, 2013). B-D) Confocal microscope images captured post-whole cell patch-clamp recording of a large soma size chick MN (solid arrow) in the ventral region of the spinal cord at E14 (HH St. 40), which was labeled using Alexa Fluor 568 dye-filled pipette. Scale bar represents $50 \mu \mathrm{m}$.

\subsubsection{Chick Kena10 DNA construct}

Dr. rer. nat. Piotr Fabrowski cloned the chick Kcna10 DNA construct. Total RNA from E5.5 (HH St. 27-28) chick embryo was purified using QIAzol Lysis Reagent (Qiagen). The embryonic cDNA library was synthesized using PrimeScript First Strand cDNA Synthesis Kit (Takara Bio Inc.) with OligodT primer following the manufacturer's protocol. Chicken Kcna10 (NP_989793), potassium voltage-gated channel subfamily A member 10, was PCR amplified from the embryonic cDNA library with primers: 
1)5'primer-ATGATGGACGTGTCCAGTTGG

2)3'primer-TTTTTTGGCCTTGTCTCGAGG

The Kcna10 coding region was inserted between CMV enhancer and the 2A peptideeGFP fusion sequence in Tol2 DNA vector.

\subsubsection{Mouse Kcna10 enhancer DNA constructs}

Dr. rer. nat. Piotr Fabrowski cloned the Kcnalo evolutionary conserved region (ECR) DNA construct. An ECR upstream of the Kcnalo promoter was identified using the ECR Browser (Ovcharenko et al., 2004). JASPAR CORE database was utilized to screen the putative binding sites for Err2/3 transcription factors (Mathelier, 2016). A 240 b.p. conserved region with three Err2/3 binding sites located 3.5 k.b. upstream of the transcription start site was chosen for further analysis: 1) AGAAGGCCA, 2) CAGAGGTCAG and 3) GCAAGGAGAT.

This region was PCR amplified from mouse genomic DNA using primers:

1) 5'primer-TCTCACAGCCCTGCTCATC

2) 3'primer-CTTGCCTGAGAACCTGATCTCC

To assess the Kcna10 ECR for activity, the ECR sequence was subsequently cloned into the Tol2-transposase system based DNA construct upstream of the minimal promoter followed by the tdTomato sequence. Furthermore, all three of the putative Err2/3 binding sites were mutated for the KcnalO ECR. The substitution mutations were introduced using site-directed mutagenesis by PCR in the Kcnalo ECR.

1. AGAAGGCCA to AGAAttaaAA

2. CAGAGGTCAG to CAtAaaTCAG

3. GCAAGGAGAT to GCAAttAGAT 
Several DNA constructs were electroporated for the Kcnal0 ECR activity assay (Fig. 2.7A-C).

A)

control (CMV-eGFP)

Kcna10 W.T. (W.T.-Kcna10

enhancer-tdTomato)

pCAGGS-T2TP

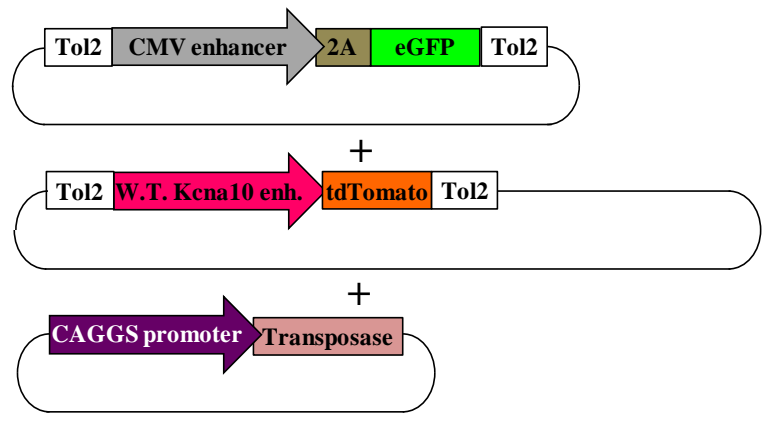

B)

Err2VP16 (CMV-VP16-Err2$e G F P)$

\section{Kena10 ${ }^{\text {W.T. }}$ (W.T.-Kcna10} enhancer-tdTomato)

pCAGGS-T2TP

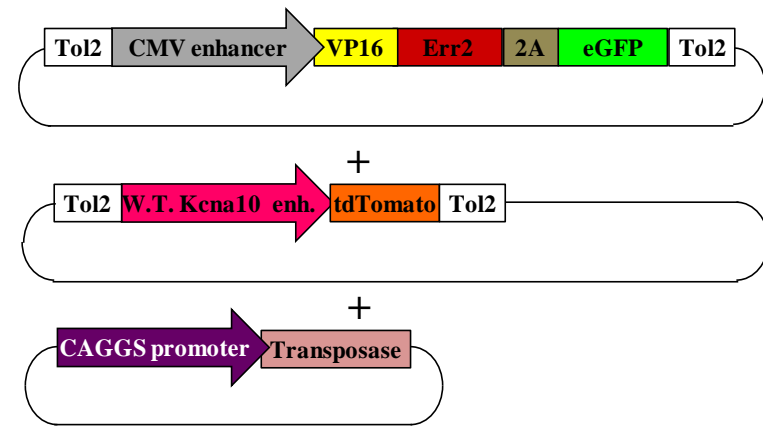

C)

Err2VP16 (CMV-VP16-Err2eGFP)

Kcna10 ${ }^{\text {mut. }}$ (W.T.-Kcna10 enhancer-tdTomato)

pCAGGS-T2TP

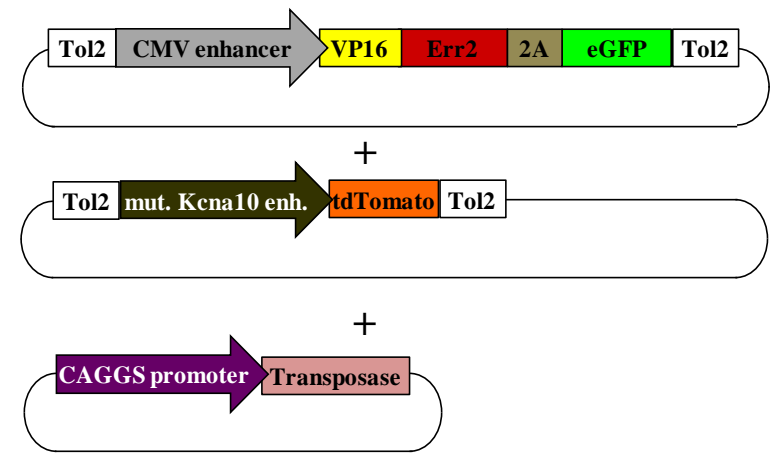

Figure 2.7 Different combinations of DNA constructs electroporated in the chick neural tube for the Kcna10 ECR activity experiments

Different DNA constructs were co-electroporated to check the effects of different constructs on the expression of tdTomato under the control of Kcnalo ECR.

\subsubsection{Chick RNA isolation and sequencing}

Chick embryos were electroporated with either control eGFP (CMV-eGFP), or Err2VP16 (CMV-VP16-Err2-eGFP), or Dlk1 ( $p C A G G S$-Dlk-1-IRES-eGFP) DNA constructs and spinal cord columns were identified by the eGFP signal, dissected and collected at E12.5 (HH St. 38-39) for the RNA sequencing studies (Fig. 2.8A-C). The RNA isolation and sequencing experiments were performed by Ashish Rajput following previous studies (Halder 
et al., 2016). RNA was isolated from the spinal columns with Tri-Reagent (Sigma-Aldrich Co. LLC, Germany), extracted using Phenol-Chloroform and resuspended in double-distilled water $\left(\mathrm{ddH}_{2} \mathrm{O}\right)$. To observe the RNA quality Nanodrop 2000 (Thermo Scientific) was used, while the Agilent 2100 Bioanalyzer (Agilent Technologies, Inc., USA) was utilized for the RNA integrity number (RIN). Subsequently, cDNA was obtained by reverse transcribing RNA with the Transcriptor High Fidelity cDNA synthesis kit (Roche Applied Science, Inc.). Several steps were taken to prepare and analyze the RNA-Seq libraries: 1) they were prepared using TruSeq RNA Sample Preparation v2 kit (Illumina, Inc.), 2) the quality was confirmed with the Agilent 2100 Bioanalyzer (Agilent Technologies, Inc., USA), 3) the concentrations were measured with Qubit ${ }^{\mathrm{R}}$ dsDNA HS Assay kit (Thermo Fisher Scientific), 4) they were normalized to $2 \mathrm{nM}$ and subsequently, sequenced (50 b.p.) on a HiSeq 2000 sequencer (Illumina, Inc.) using TruSeq SR Cluster kit v3-cBot-HS (Illumina, Inc.) and TruSeq SBS kit v3-HS (Illumina, Inc.).

A)

control (CMV-eGFP)

pCAGGS-T2TP

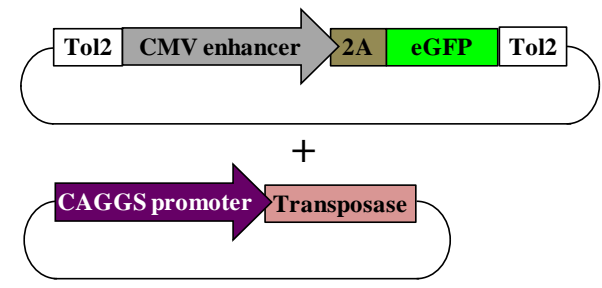

B)

Err2VP16 (CMV-VP16-Err2eGFP)

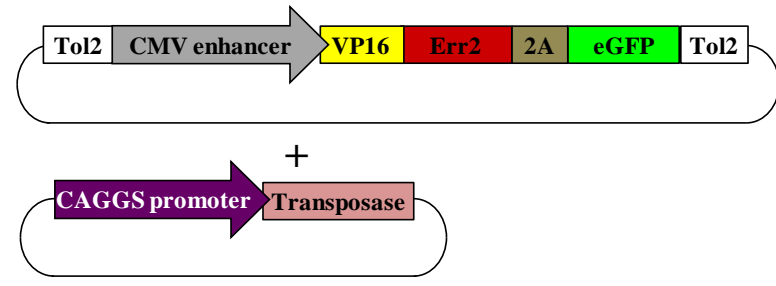

C)

Dlk1 (pCAGGS-Dlk1-IRESeGFP)

pCAGGS-T2TP

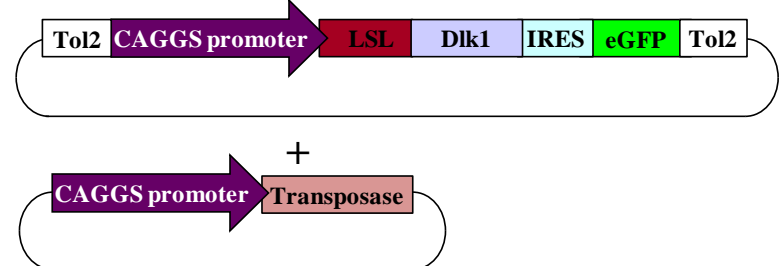

pGK-Cre

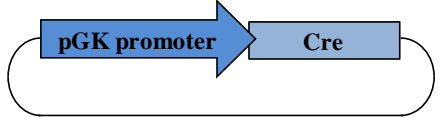

Figure 2.8 Different combinations of DNA constructs co-electroporated in the chick neural tube for the chick RNA sequencing studies

Different constructs were co-electroporated for the chick RNA sequencing experiments. The LSL is a loxSTOPlox cassette in the Dlk1 ( $p C A G G S$-Dlkl-IRES-eGFP) DNA construct that is excised in Cre expressing cells. 


\subsubsection{Chick RNA sequencing analysis}

RNA sequencing analysis of the chick spinal column experiments was performed by Dr. Vikas Bansal, Ph.D. Raw reads were used to determine the RNA-sequencing quality with the FastQC quality control tool version 0.10.1 (Andrews, 2010). To align the sequence reads (single-end 50 b.p.) to reference genome in chick (Galgal5), Bowtie2 (v2.0.2) with RSEM (v1.2.29) with the provided settings were used (Langmead and Salzberg, 2012; Li and Dewey, 2011). First, in the reference genome fasta file and annotation GTF file, sequences and annotations of GFP, Err2, VP16, Dlk-1, and IRES were added. Then to index the chick genome, Ensembl annotations (version 86.5) with rsem-prepare-reference was utilized in the RSEM software (Yates et al., 2015). Then to align sequence reads and quantify genes, rsemcalculate-expression was used to obtain a transcript per million (TPM) for single genes. Differential expression analysis was performed after obtaining gene read counts using DESeq2 package (Love et al., 2014). Finally, genes were filtered $<5$ reads (baseMean) and classified as differentially expressed ( $p$-value $<0.05)$.

\subsection{Chick and mouse electrophysiology}

\subsubsection{Chick spinal cord slice preparation}

Chick embryos were allowed to develop until E12-15 (HH stages 38-41). Thoracolumbar spinal cord regions with the vertebral column were dissected in chilled $\mathrm{DaCSF} / \mathrm{RaCSF}$ solution (Table 2.8). To acquire slices for recordings, this region was inserted into a columnar agarose block (4\% agarose mixed in DaCSF/RaCSF solution) and stabilized with a solidifying $20 \%$ gelatin (20\% gelatin mixed in DaCSF/RaCSF solution). Thoracolumbar slices of $370 \mu \mathrm{m}$ thickness were dissected using the Leica VT1200 S (Leica Biosystems, $\mathrm{GmbH})$ and incubated in $\mathrm{DaCSF} / \mathrm{RaCSF}$ for $30 \mathrm{~min}$ at $\mathrm{RT}\left(22^{\circ} \mathrm{C}\right)$ to recuperate from any injury and subsequently, recorded.

\subsubsection{Chick whole cell patch-clamp recordings}

DNA constructs were electroporated into chick neural tube for the chick electrophysiology recordings (Fig. 2.9A-G). 
A)

control (CMV-eGFP)

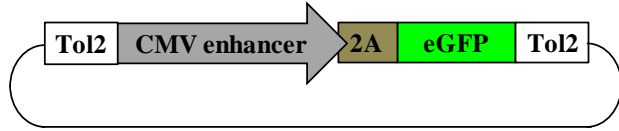

B)

Err2 (CMV-Err2-eGFP)

C)

Err3 (CMV-Err3-eGFP)
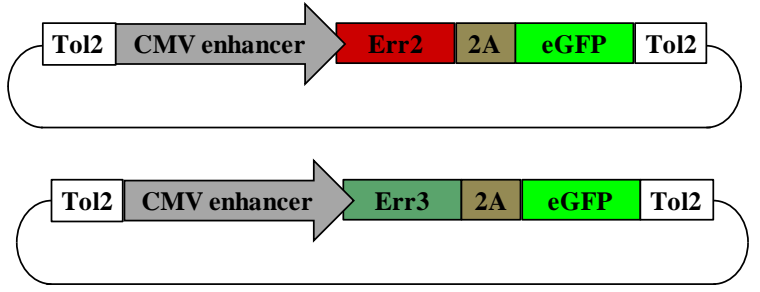

D)

Err2VP16 (CMV-VP16-Err2eGFP)

E)

Err2EnR (CMV-EnR-Err2eGFP)

F)

Kena10 (CMV-Kcna10eGFP)

G)

pCAGGS-T2TP
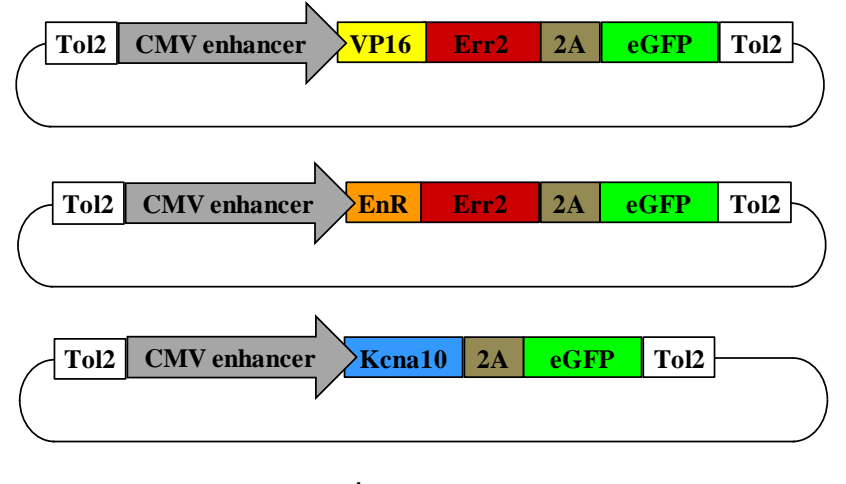

\section{CAGGS promoter Transposase}

Figure 2.9 DNA constructs electroporated in chick neural tube for chick the whole cell patchclamp recordings

The following constructs were electroporated in the chick neural tube E2.7-3.0 (HH St. 14-18) for the whole cell patch-clamp recordings. Constructs A-F were co-electroporated with a helper DNA construct pCAGGS-T2TP carrying the transposase gene. Transposase recognizes the Tol2 sequences (flanking the DNA constructs A-F) and catalyzes the transposition of DNA inserts between the two Tol2 sites (Section 2.13.1).

For the recordings, large soma size MNs in the ventral horn of the spinal cord were observed using GFP expression on an Olympus BX51W1 microscope installed with 4x air objective (Olympus UPlan FL N) and 40x water-immersion objective (Olympus UPlan FLN). Pipette with resistances between 3-6 M $\Omega$ were used to record MNs. To identify the recorded MNs, $25 \mu \mathrm{M}$ Alexa Fluor 568 dye (Thermo Fisher Scientific) was also added to the intracellular solution.

The recording signals obtained in current-clamp were amplified and filtered with a MultiClamp 700B patch-clamp amplifier (Molecular Devices). The signal was acquired at 20 $\mathrm{kHz}$ with the Digidata 1322A digitizer (Molecular Devices) and pCLAMP 10.4 software (Molecular Devices). 


\subsubsection{Mouse spinal cord slice preparation}

The preparation of spinal cord tissue and solutions followed a similar procedure to Mitra and Brownstone, (2012). To anesthetize mice, control and Err2/3 ${ }^{c K O}$ mice at postnatal day (P20-P22) were intraperitoneally injected and observed until they lost their righting reflex. Then they placed on ice until they were unable to react to a toe pinch $(\sim 5 \mathrm{~min})$. The mice were decapitated, eviscerated swiftly and to clear the torso of blood and body fluids, the torso was placed in chilled Dissecting aCSF (DaCSF) solution (Table 2.8 in section 2.8). The torso was fully submerged and pinned in a Sylgard petri dish containing DaCSF and vertebrectomy was performed to extract the spinal cord, ventral roots were cut and the meninges were removed to clean the spinal cord. The thoracolumbar portion (T10-L5) of the spinal cord was dissected and embedded in a columnar agar block (4\% agarose in Recording aCSF $(\mathrm{RaCSF})$ ) and stabilized in the agararose block with $20 \%$ gelatin (20\% gelatin in RaCSF). Spinal cord slices of $370 \mu \mathrm{m}$ thickness were dissected with the Leica VT1200 S (Leica Biosystems, GmbH). The slices were incubated at $35^{\circ} \mathrm{C}$ in $\mathrm{RaCSF}$ for $30 \mathrm{~min}$ and incubated 30 min at $\mathrm{RT}\left(22^{\circ} \mathrm{C}\right)$ in RaCSF to recuperate from injury until recordings. MNs were recorded in RaCSF solution (Table 2.8 in section 2.8).

\subsubsection{Mouse whole cell patch-clamp recordings}

Whole cell patch-clamp recordings were performed in FG labeled MNs in the ventral horn from control and Err2/3 ${ }^{c K O}$ mice at P20-P22. At this developmental stage, the $\gamma-\mathrm{MNs}$ have mature morphology in mice (Fig. 2.10). $\mathrm{FG}^{\text {high }}$ and $\mathrm{FG}^{\text {low }} \mathrm{MNs}$ were identified visually with Olympus BX51W1 microscope equipped with an FG longpass filter set (350 Bandpass and 425 Longpass-Filter) (AHF analysentechnik AG, Tübingen Germany). The identified MNs were recorded with a pipette that had resistances of 3-6 $\mathrm{M} \Omega$. 


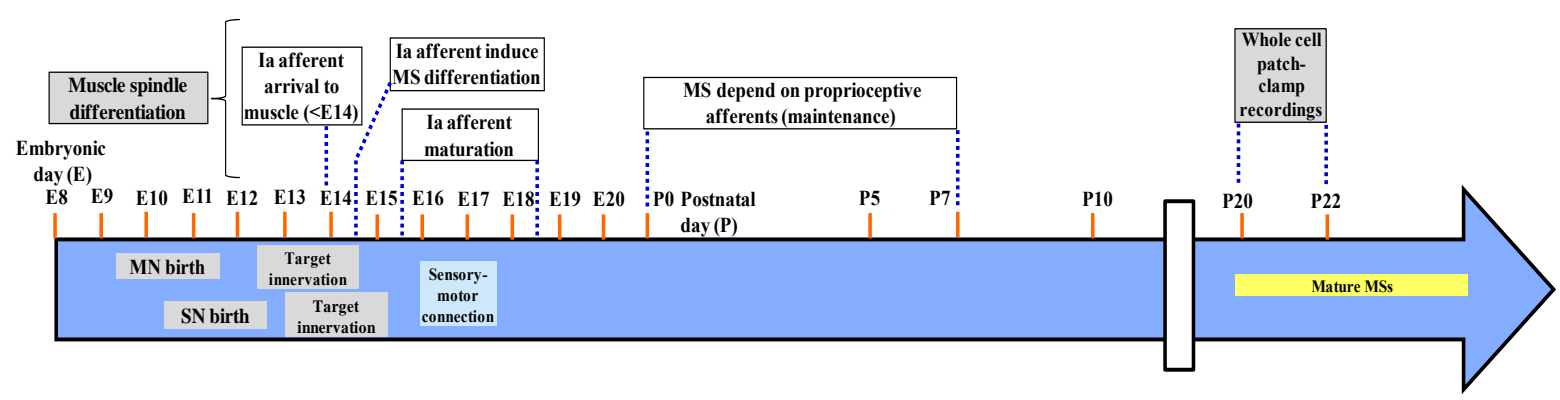

Figure 2.10 MN, SN and MS differentiation in mice

A developmental time-line in approximate embryonic and postnatal days marking motor neuron $(\mathrm{MN})$, sensory neurons $(\mathrm{SN})$ and muscle spindles (MS) differentiation (indicated by a gradient). Whole cell patch-clamp recordings were performed in mature $\gamma$-MNs that innervate MSs with mature morphology. (Adapted from Ladle et al., 2009; Hippenmeyer et al., 2002; Chen and Frank, 1999; Sonner et al., 2017)

\subsubsection{Chick and Mouse whole cell patch-clamp analysis}

To analyze the biophysical properties such as rheobase, F-I curve, AHP amplitude, half-width and half-decay times, input resistance and capacitance, protocols from previous studies were used for the recordings and the data was analyzed offline with Axograph $\mathrm{X}$ Version 1.6 software (AxoGraph Software) (Mitra and Brownstone, 2012; Müller et al., 2014; Miles et al., 2004; Miles et al., 2005; Nakanishi and Whelin, 2010; Muramoto et al., 1996). To analyze the discharge properties, action potentials were evoked by injecting increments of $20 \mathrm{pA}, 1000 \mathrm{~ms}$ square current pulses to MNs to generate an entire $F$ - $I$ curve. For chick MN recordings, all neurons were subjected to increments of 20 pA, $1000 \mathrm{~ms}$ pulses (up to $1 \mathrm{nA}$ ) of current during the $F-I$ curve protocol (Fig. 2.11A-C). The $F-I$ curve acquired for MNs from control and $E r r 2 / 3^{c K O}$ mice, MNs that incorporated high FG were subjected to increments of $20 \mathrm{pA}, 1000 \mathrm{~ms}$ pulses up to $1 \mathrm{nA}$ of current (since higher currents caused a firing block and highly unstable firing), while MNs that incorporated low FG were subjected to increments of $20 \mathrm{pA}, 1000 \mathrm{~ms}$ pulses up to $3 \mathrm{nA}$ of current. Similar to previous studies, the firing frequency $(\mathrm{Hz})$ of a $\mathrm{MN}$ was calculated by taking the inverse of the time or duration between two action potentials, while mean firing frequency with respect to the current input generates a regression line whose slope is taken as the gain (Hz/nA) (Manuel et al., 2009; Hadzipasic et al., 2014). The neurons were placed into bins according to the initial onset of repetitive firing and a similar rate of current change was used to obtain the data points for firing frequency and gain. Three types of gain were determined according to the regularity of firing pattern during the F-I curve protocol: 1) gain, 2) gain (steady-state) and 3) gain (instantaneous) (Fig. 2.12A). The gain was calculated as the slope of the firing frequency for 
the entire duration (1 s) of the pulse (Fig. 2.12B). The gain (steady-state) was calculated as the slope of mean firing frequency from stable regions of firing consisting of $250 \mathrm{~ms}$ to 750 ms of the pulse (Fig. 2.12B). The gain (instantaneous) was calculated by obtaining the slope of firing frequency from the first 2 spikes elicited (Fig. 2.12B).

A)

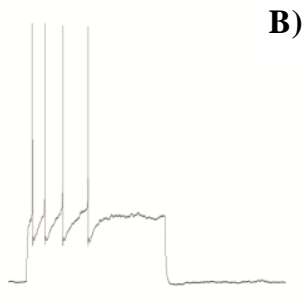

B)

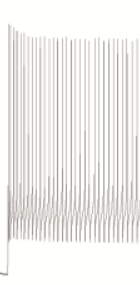

(1)

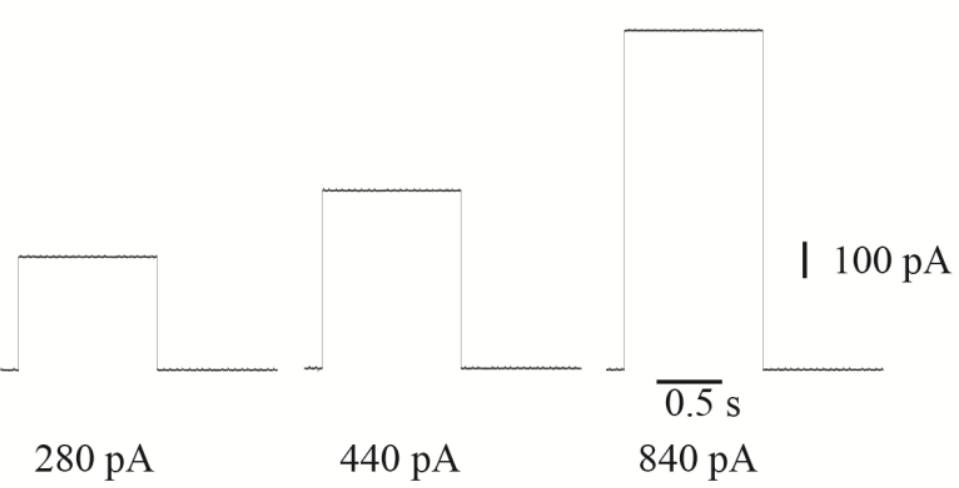

Figure 2.11 F-I traces for calculating firing frequency and gain

(A-C) For studying the discharge properties of MNs, F-I curves were attained by eliciting action potentials by injecting current increments of $20 \mathrm{pA}, 1000 \mathrm{~ms}$. A voltage response trace with current injections shows that repetitive firing was elicited in the FG $^{\text {high }}$ putative $\gamma$-MN. The pulse duration, amplitude and current injection are shown with scale bars that represent $0.5(\mathrm{~s}), 20 \mathrm{mV}, 100 \mathrm{pA}$, respectively. 
A)

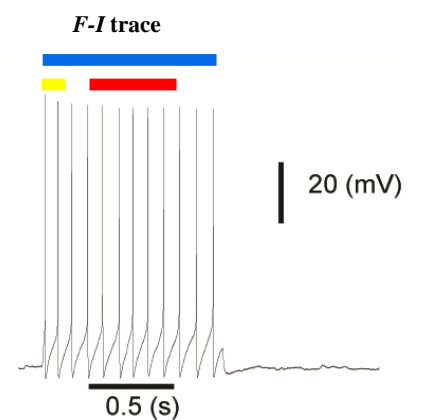

Definitions:

firing frequncy $[\mathrm{Hz}]:$ : Interspike interval

gain $[\mathrm{Hz} / \mathbf{n A}]$ : current injection versus mean firing

frequency. Slope of Regression line.

- gain and firing frequency (entire $1 \mathrm{~s}$ interval)

- gain and firing frequency (steady-state) $(0.25 \mathrm{~s}-0.75 \mathrm{~s}$ interval)

gain and firing frequency (instantaneous) (first interspike interval)

B)
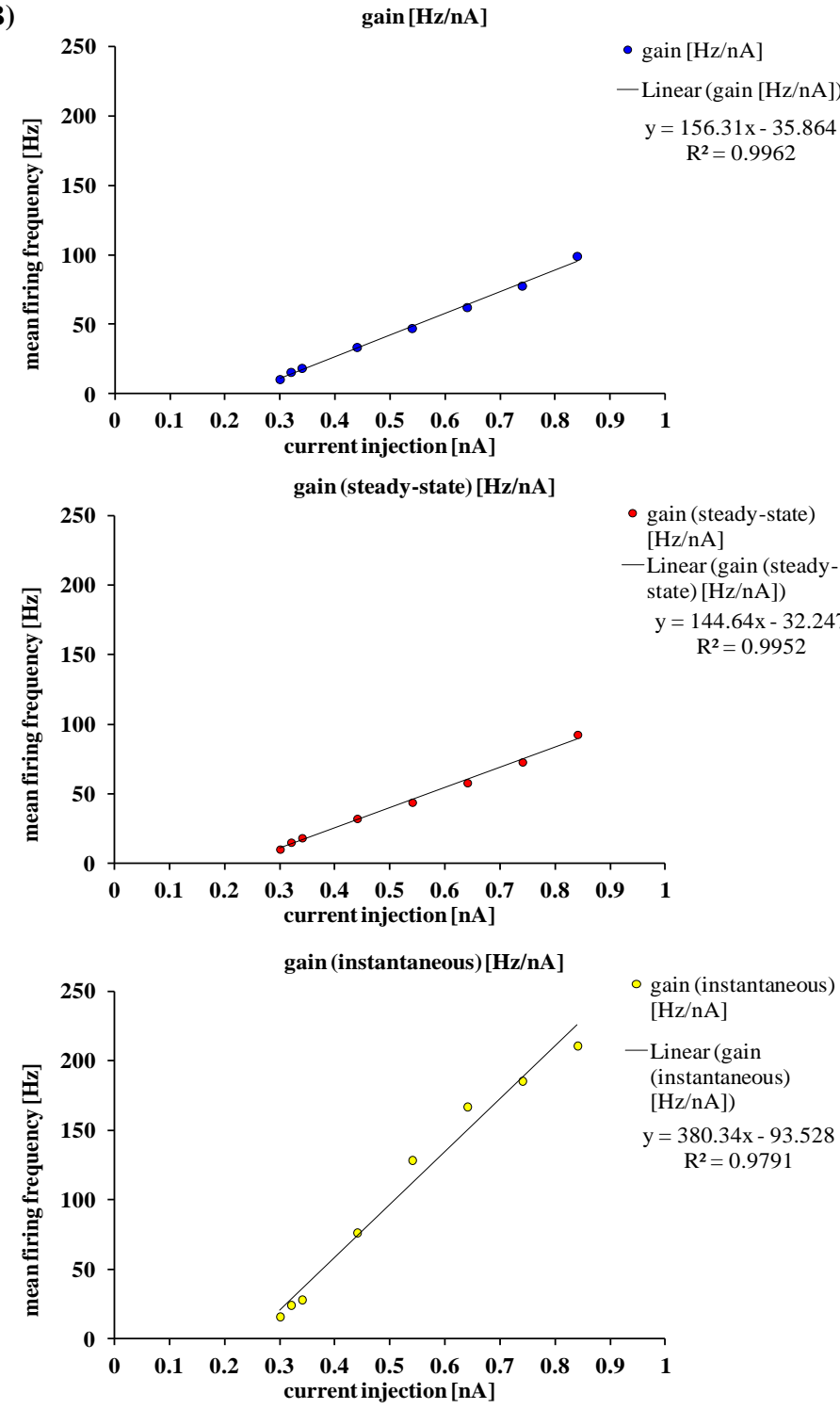

Figure 2.12 Calculation of gain and firing frequency from $F$-I curve

A) The firing frequency is defined as the 1/interspike interval and the gain captures the relationship of how firing frequency changes with current injection, which is calculated as the slope of the firing frequency plot. Gain spans $1 \mathrm{~s}$ and is marked by the blue bar. Gain (steady-state) spans $0.5 \mathrm{~s}$ and is marked by the red bar. Finally, gain (instantaneous) spans the first interspike interval is marked by the yellow bar. B) The mean firing frequency from these regions was plotted with respect to current injection and the slope of the best fit line was used to calculate the gain (1s), gain (steady-state) and gain (intantaneous). 


\subsection{Bacterial transformation and DNA extraction}

In general, the molecular biology methods were carried out according to Sambrook and Russell, 2001, if not specified. E. coli cells (One Shot TOP10 strain) (Thermo Fisher Scientific) were prepared and stored at $-80^{\circ} \mathrm{C}$ according to previously published protocols (Inoue, 1990). In order to transform the bacteria, the ligated reaction was mixed and incubated for $30 \mathrm{~min}$ on ice with $100 \mu \mathrm{l}$ of chemically competent $E$. coli cells. The cells were then incubated on a block at $42^{\circ} \mathrm{C}$ for 1 min to heat shock and then transferred back to the ice for $1 \mathrm{~min}$ and $1 \mathrm{ml}$ of antibiotic-free LB medium was added. To allow the transformed bacteria to grow, they were incubated on thermomixer for $1 \mathrm{~h}$ with the settings of 1000 r.p.m at $37^{\circ} \mathrm{C}$, then centrifuged at 4000 r.p.m. for $3 \mathrm{~min}$ and plated on agar plates treated with Ampicillin after being disposed of their supernatant. The plates were then incubated overnight (O.N.) at $37^{\circ} \mathrm{C}$ for the bacteria to grow. Single colonies of bacteria were selected with a toothpick or pipette tip and incubated in $15 \mathrm{ml}$ of LB medium containing Ampicillin and placed a shaker O.N. with settings of 180 r.p.m. at $37^{\circ} \mathrm{C}$ to grow clones. Subsequently, $500 \mu \mathrm{l}$ of $E$. Coli clones were then vortexed with $500 \mu \mathrm{l}$ of glycerol in $1.5 \mathrm{ml}$ Eppendorf tubes and stored at $-80^{\circ} \mathrm{C}$ as cryostocks.

E. coli cells were regrown in $15 \mathrm{ml}$ of LB medium containing Ampicillin for smaller quantities of DNA extraction (extracted using QIAprep spin miniprep kit) (Qiagen GmbH) and $200 \mathrm{ml}$ of LB medium containing Ampicillin for larger quantities of DNA extraction (extracted using QIAGEN DNA construct Maxiprep Kit). DNA was eluted in $30 \mu 1$ and 50 $\mu l$ of elution buffer and appropriate concentrations were adjusted for the experiments. The DNA construct concentrations were measured using Nanodrop 1000 UV-Vis Spectrophotometer (Thermo Fisher Scientific GmbH). DNA was separated via gel electrophoresis and purified using QIAquick Gel Extraction Kit (Qiagen GmbH) using manufacturer's protocol. DNA was sequenced using SEQLAB: Sequence laboratories Goettingen, GmbH.

\subsection{Statistical analysis}

Quantification was presented as mean \pm SEM (standard error of the mean) and statistical comparison of the quantitative immunodetection, the behavior and the 
electrophysiology experiments was determined by using a two-tailed Student's $t$-test assuming unequal variance except when specified differently. 


\section{RESULTS}

\subsection{Characterization of Err2 and Err3 transcription factor expression pattern in wild-type mouse spinal cord}

Recent studies showed that Err3 expression becomes limited to $\gamma$-MNs in mice by postnatal day 14 (P14) (Friese et al., 2009). The closely related Err2 has been suggested to be a molecular correlate for slow $\alpha-M N s$ (Enjin et al., 2010). Thus, I reexamined Err2 and Err3 expression patterns in wild-type mouse spinal cords during early postnatal development.

\subsubsection{Err2 and Err3 expression pattern during early postnatal development}

To reexamine Err2 and Err3 developmental expression patterns, wild-type (W.T.) (CD1 strain) mice were intraperitoneally injected with Fluoro-Gold (FG), a retrograde tracer taken up by MNs and used to label MNs (Material \& Methods section 2.12.3). For immunodetection studies, cryosections from the thoracolumbar region of the spinal cord were incubated with primary antibodies raised against Err2, Err3 and $\mathrm{NeuN}$ and appropriate secondary antibodies and imaged using a confocal microscope (Materials \& Methods section 2.12.5) (Materials \& Methods Table 2.1 and 2.2 in section 2.1 and 2.2, respectively). The anti-Err2 and anti-Err3 primary antibodies specifically recognize Err2 (Fig. 7.1A-F in Appendix 1) and Err3 (Fig. 7.1G-L in Appendix 1), respectively. Moreover, IgG2b and IgG2a isotype-specific secondary antibodies specifically recognize Err2 (Fig. 7.2A-H in Appendix 1) and Err3 (Fig. 7.2I-P in Appendix 1), respectively. In agreement with the findings by Friese et al., (2009), relatively small soma size putative $\gamma$-MNs expressed high levels of Err3 (Fig. 3.1B) and low levels of NeuN (Fig. 3.1C). Furthermore, I observed that these putative $\gamma$-MNs expressed high levels of Err2 (Fig. 3.1A) and incorporated high levels of FG in their soma (Fig. 3.1D), while putative $\alpha$-MNs expressed low levels of Err2 (Fig. 3.1A) and Err3 (Fig. 3.1B), moderate-to-high levels of NeuN (Fig. 3.1C), and incorporated low levels of FG in their soma (Fig. 3.1D). Taken together, I observed that at P21, putative $\gamma$ MNs co-express high levels of Err2 and Err3, low levels of NeuN and incorporate high levels of FG (Fig. 3.1A-E). 

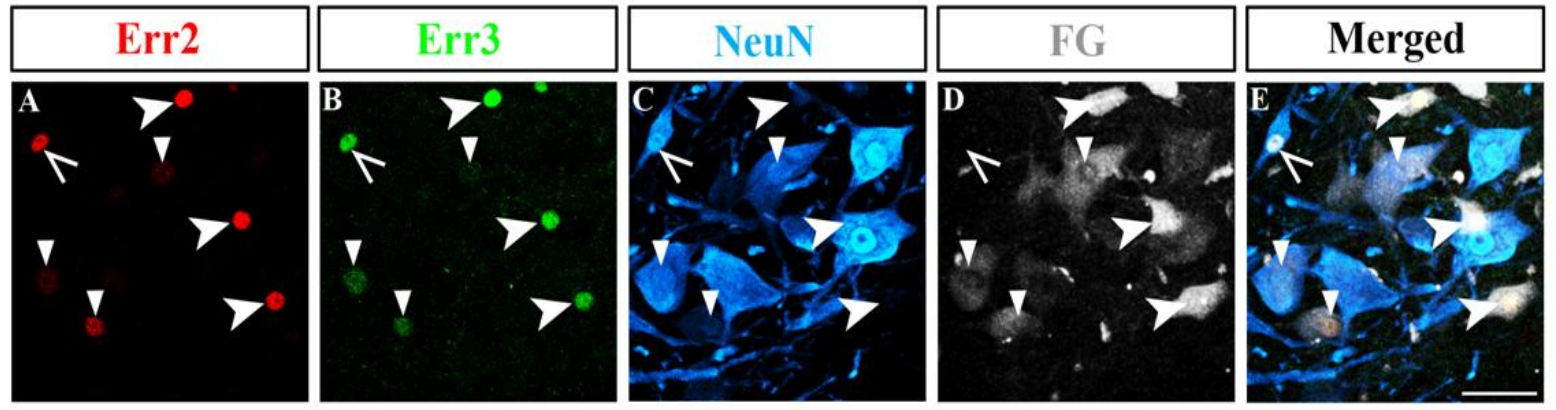

Figure 3.1 Co-expression of high levels of Err2 and Err3 in relatively small soma size putative $\gamma$ MNs that express low levels of NeuN and incorporate high levels of FG at P21

(A-E) Confocal images showing the expression pattern of Err2 (red, nuclei), Err3 (green, nuclei), NeuN (blue, soma and nuclei), FG (gray, soma), in thoracolumbar cryosections from P21 W.T. mice. Solid arrows indicate putative $\gamma$-MNs with the expression levels of Err $2^{\text {high }}$, Err $3^{\text {high }}$, NeuN ${ }^{\text {low }}$, while incorporating high levels of FG. Triangles indicate putative $\alpha$-MNs with the expression levels of Err $2^{\text {low }}, \mathrm{Err}^{\text {low }}{ }^{\text {, NeuN }}{ }^{\text {moderate-to-high }}$, that incorporate low levels of FG. Hollow arrow indicates noncholinergic cell with the expression levels of Err $2^{\text {high }}, \mathrm{Err}^{\text {high }}{ }^{\mathrm{Neu}} \mathrm{N}^{\text {high }}$, that incorporate no FG. Scale bar represents $50 \mu \mathrm{m}$.

The developmental expression patterns of Err2 and Err3 is shown at P4 (Fig. 3.2A-E), P14 (Fig. 3.2F-J) and P21 (Fig. 3.2K-O) in W.T. mice. Furthermore, the developmental expression pattern of Err2 and Err3 was quantified in MNs during the early postnatal ages at P4 (Fig. 3.3A, B), P14 (Fig. 3.3C, D), and P21 (Fig. 3.3E, F). From P4 onwards MNs with a soma cross-sectional area of $\sim 250 \mu \mathrm{m}^{2}-500 \mu \mathrm{m}^{2}$ showed significantly higher expression levels of nuclear Err2 and Err3 compared to MNs with soma sizes $>500 \mu \mathrm{m}^{2}$ (P4: Fig. 3.3A, B; $n=100, N=6$; P14: Fig. 3.3C, D; $n=189, N=6$ ). This segregated expression pattern became more pronounced up to P21 (Fig. 3.3E, F; n=159, N=6). Err2 and Err3 are therefore coexpressed at high levels in relatively small soma size MNs that expressed low levels of NeuN and incorporated high levels of FG, hence, suggesting that high Err2 and Err3 levels are able to distinguish $\gamma$-MNs from other MNs (Fig. 3.2A-O; Fig. 3.3A-F). 

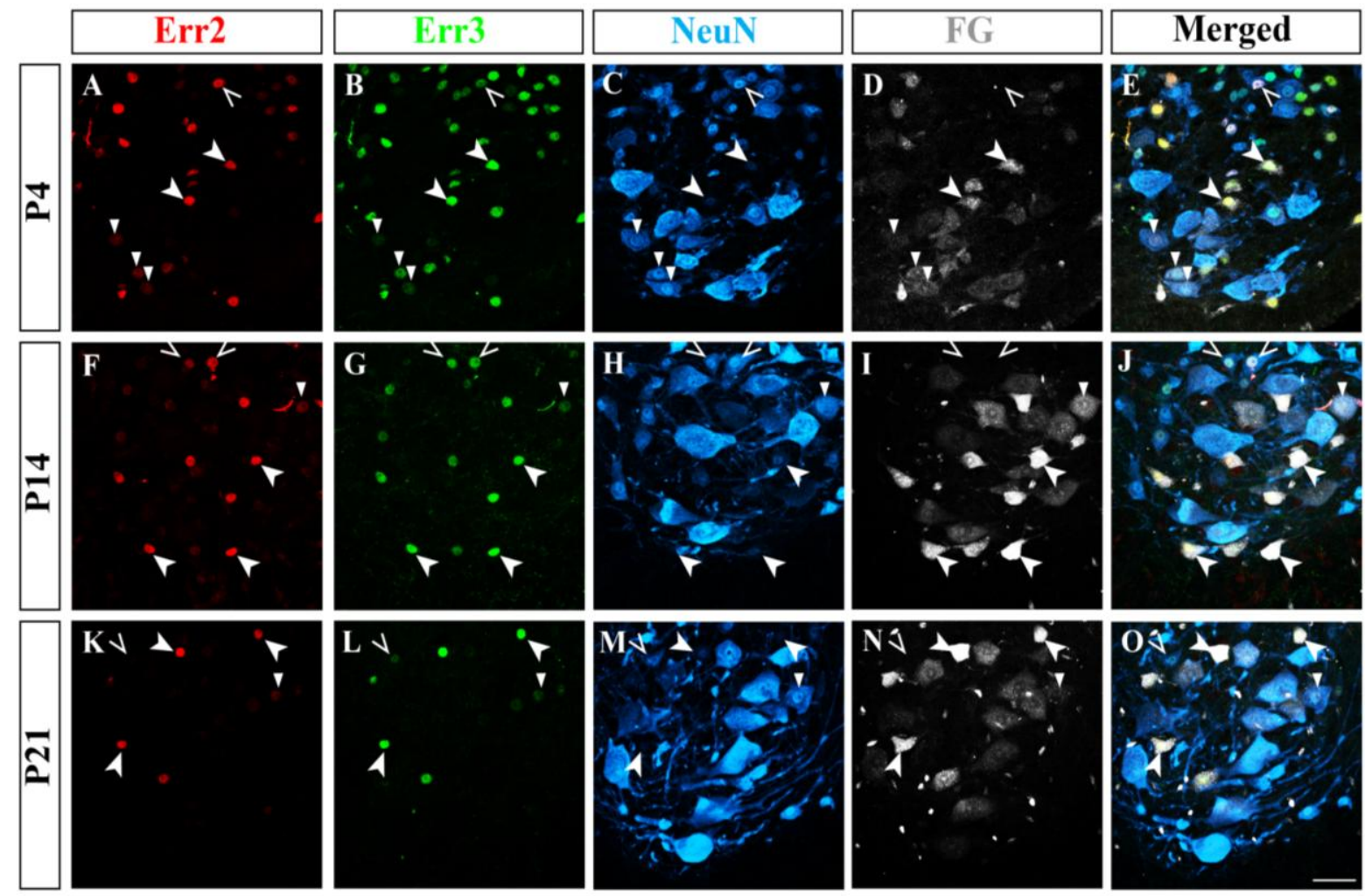

Figure 3.2 High levels of Err2 and Err3 expression becomes more restricted to relatively small soma size MNs that express low levels of NeuN and incorporate high levels of FG at P21

(A-O) Confocal images showing Err2 (red, nuclei), Err3 (green, nuclei), NeuN (blue, soma and nuclei), FG (gray, soma), and Merged during early postnatal stages of P4 (A-E), P14 (F-J), and P21 (K-O). Solid arrows indicate putative $\gamma$-MNs with the expression levels of Err $2^{\text {high }}, \mathrm{Err}^{\text {high }}{ }^{\text {, }} \mathrm{NeuN}^{\text {low }}$ and incorporation of high FG levels. Triangles indicate putative $\alpha$-MNs with the expression levels of Err $2^{\text {low }}, \mathrm{Err}^{\text {low }}{ }^{\text {, NeuN }}{ }^{\text {moderate-to-high }}$ and incorporation low FG levels. Hollow arrows indicate noncholinergic cells with the expression levels of Err2 ${ }^{\text {high }}, \mathrm{Err}^{\text {high }}, \mathrm{NeuN}^{\text {high }}$ and lack of any FG incorporation. Scale bar represents $50 \mu \mathrm{m}$. 
A)
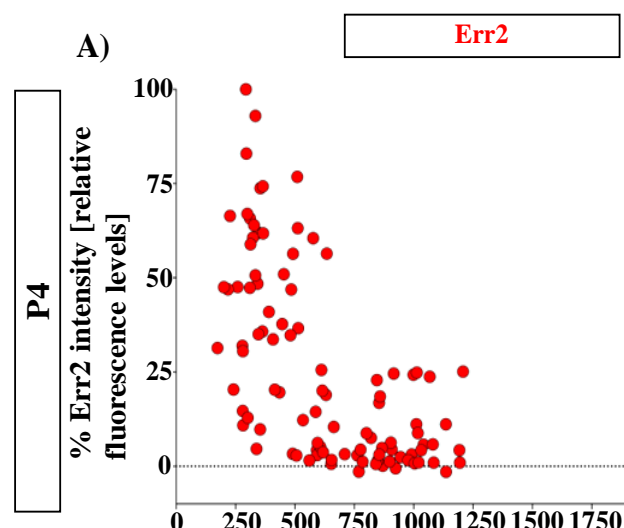

C)

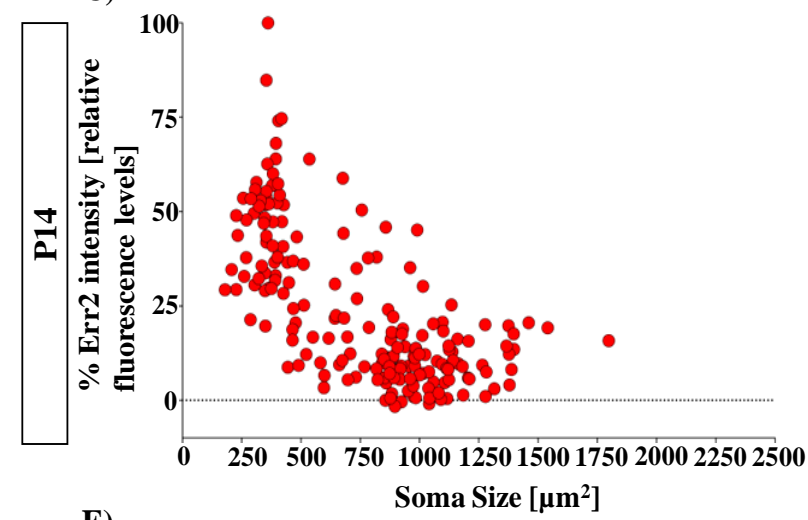

E)

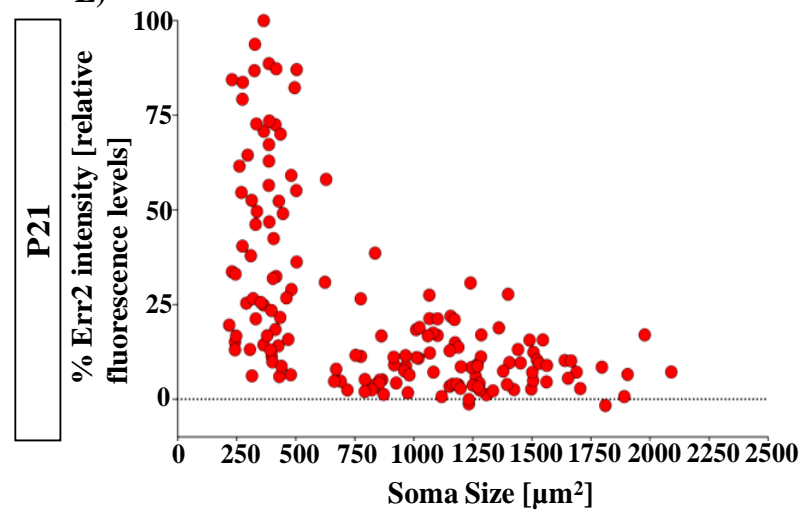

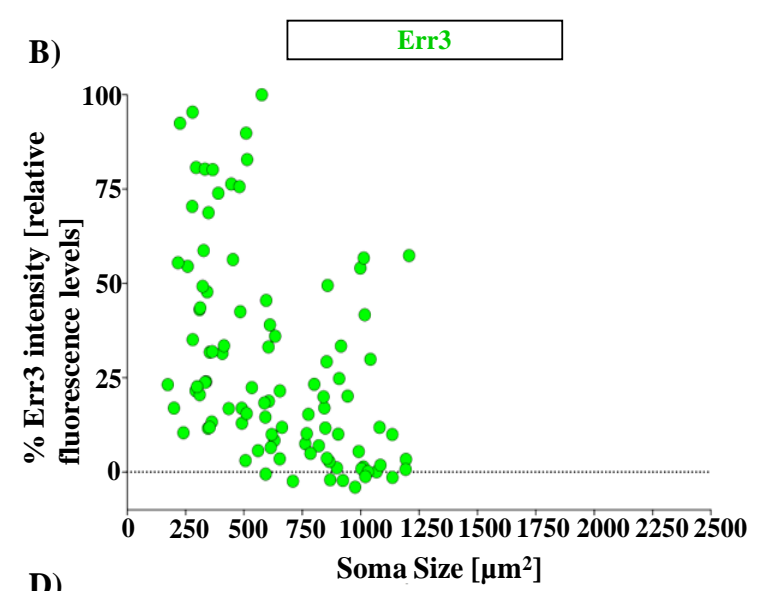

D)
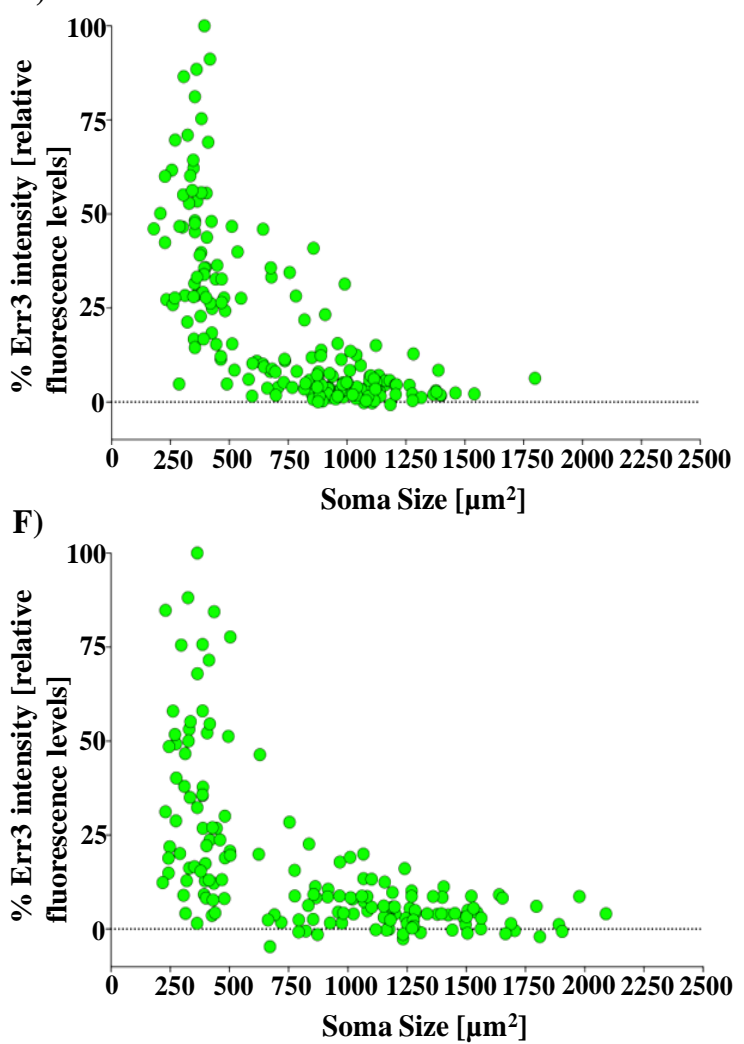

Figure 3.3 High expression levels of Err2 and Err3 distinguish relatively small soma size putative $\gamma$-MNs as development proceeds

(A-F) Scatter-plots of Err2 and Err3 normalized fluorescent intensity at P4 (A, B), P14 (C, D), P21 (E, F). X-axis depicting soma size in $\mu \mathrm{m}^{2}$ and $\mathrm{y}$-axis depicting normalized $\%$ fluorescent intensity in relative fluorescence levels. The relatively small soma size MNs $\left(\sim 250-500 \mu \mathrm{m}^{2}\right)$ show high Err2 and Err3 expression levels and the relatively large soma size MNs $\left(600 \mu \mathrm{m}^{2}-2000 \mu \mathrm{m}^{2}\right)$ show low Err2 and Err3 expression levels. A segregation in Err2 and Err3 expression pattern is observable at P21.

\subsection{Err2 and Err3 expression pattern in Err2/3 ${ }^{c K O}$ mouse spinal cord}

Previous studies in the host laboratory suggested that Err2 conditional knockout $\left(E r r 2^{c K O}\right)$ mice lacking Err2 in MNs showed no abnormal phenotype when they were subjected to the grip strength and running endurance tasks (Lee, 2013). Moreover, the sequence similarities and the co-expression of Err2 and Err3 in putative $\gamma$-MNs suggested 
that Err2 and Err3 may be functionally redundant. To determine the role of Err2 and Err3 in the development of $\gamma$-MN functional properties, I intended to generate mice lacking Err2 and Err3 in $\gamma$-MNs. To generate conditional knockout (cKO) mice, I used the Cre/loxP system, which can be employed for cell-specific inactivation of gene in mice (Sauer and Hendersen, 1988; Hall et al., 2009). To achieve this, I used the ChAT-IRES-Cre knockin (Chat ${ }^{\mathrm{Cre}}$ ) mouse line, because it is known that cholinergic cells, including MNs, use Acetylcholine as their neurotransmitter, thus, express choline acetyltransferase (ChAT), an enzyme that catalyzes the reaction in Acetylcholine synthesis (Fig. 2.1A, B in Materials \& Methods section 2.10) (Rossi et al., 2011). Upon interbreeding the Chat ${ }^{C r e}$ and "floxed" Esrrb allele mice with "floxed" Esrrg allele mice, a mouse line with cKO of Err2 and Err3 MNs was generated (Fig. 2.3A, B in Materials \& Methods section 2.10).

Using quantitative immunodetection studies, I verified whether the Cre/loxP system led to a successful deletion of Esrrb and Esrrg genes in the putative $\gamma$-MNs of Err2/3 ${ }^{\text {cKO }}$ mice. Spinal cord cryosections of FG injected mice were incubated with specific anti-Err2 and anti-Err3 primary antibodies and appropriate isotype-specific secondary antibodies and imaged using a confocal microscope. The putative $\alpha$-MNs from control (C57BL/6 strain) mice expressed low levels of Err2 and Err3 (Fig. 3.4A, B), moderate-to-high levels of NeuN (Fig. 3.4C), and incorporated low levels of FG (Fig. 3.4D). While the putative $\gamma$-MNs from control mice expressed high levels of Err2 and Err3 (Fig. 3.4A, B), low levels of NeuN (Fig. 3.4C), and incorporated high levels of FG (Fig. 3.4D). While the putative $\gamma$-MNs from Err $2 / 3^{c K O}$ that incorporated high levels of FG (Fig. 3.4I) and expressed low levels of NeuN (Fig. 3.4H), lacked any Err2 and Err3 (Fig. 3.4F, G). The quantification showed that the expression levels of Err2 and Err3 in MNs from control mice (Fig 3.5A, B; n=125, N=6) was significantly different than the expression levels in MNs from Err2/3 ${ }^{c K O}$ mice (Fig. 3.5C, D; $\mathrm{n}=145, \mathrm{~N}=4 ; p=5.02 \mathrm{e}^{-12}, p=4.84 \mathrm{e}^{-26}$, respectively). Thus, the data showed that the expression of Err2 and Err3 was successfully eliminated in the putative $\gamma$-MNs from Err2/3 ${ }^{c K O}$ mice (Fig. 3.4A-J; Fig. 3.5A-D). 


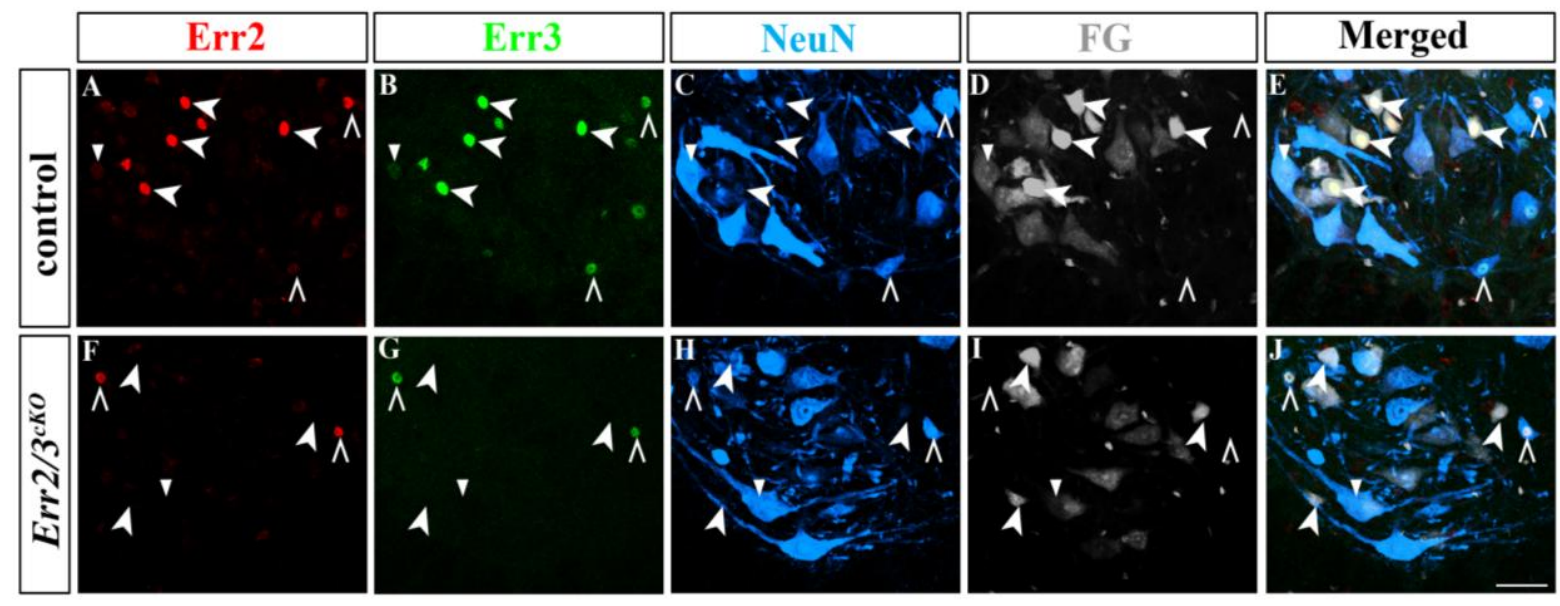

Figure 3.4 Elimination of Err2 and Err3 expression from MNs in Err2/3 ${ }^{c K O}$ mice

(A-J) Representative confocal microscope images showing the ventral horn spinal cord from control and $E r r 2 / 3^{c K O}$ mice. The spinal cord cryosections were incubated with anti-Err2, anti-Err3 and antiNeuN primary antibodies and appropriate isotype-specific secondary antibodies. (A-E) Control mice have high expression levels of Err2 and Err3 in putative $\gamma$-MNs (indicated by solid arrows), which also have low expression levels of NeuN and incorporate high levels of FG. Conversely, putative $\alpha-$ MNs (indicated by triangle) have low expression levels of Err2 and Err3, express moderate-to-high levels of NeuN and incorporate low levels of FG. And non-cholinergic cells (indicated by hollow arrows) express high levels of Err2 and Err3, high expression levels of NeuN and do not incorporate FG. (F-J) $E r r 2 / 3^{c K O}$ mice lack any expression of Err2 and Err3 in putative $\gamma$-MNs (noted by solid arrows), which also have low expression levels of NeuN and incorporate high levels of FG. Putative $\alpha$-MNs (noted by triangle), lack any expression of Err2 and Err3 as well, which express moderate-tohigh levels of NeuN and incorporate low levels of FG. While non-cholinergic cells (noted by hollow arrows) have preserved expression levels of Err2 and Err3, have high expression levels of NeuN and incorporate no FG. Scale bar represents $50 \mu \mathrm{m}$. 

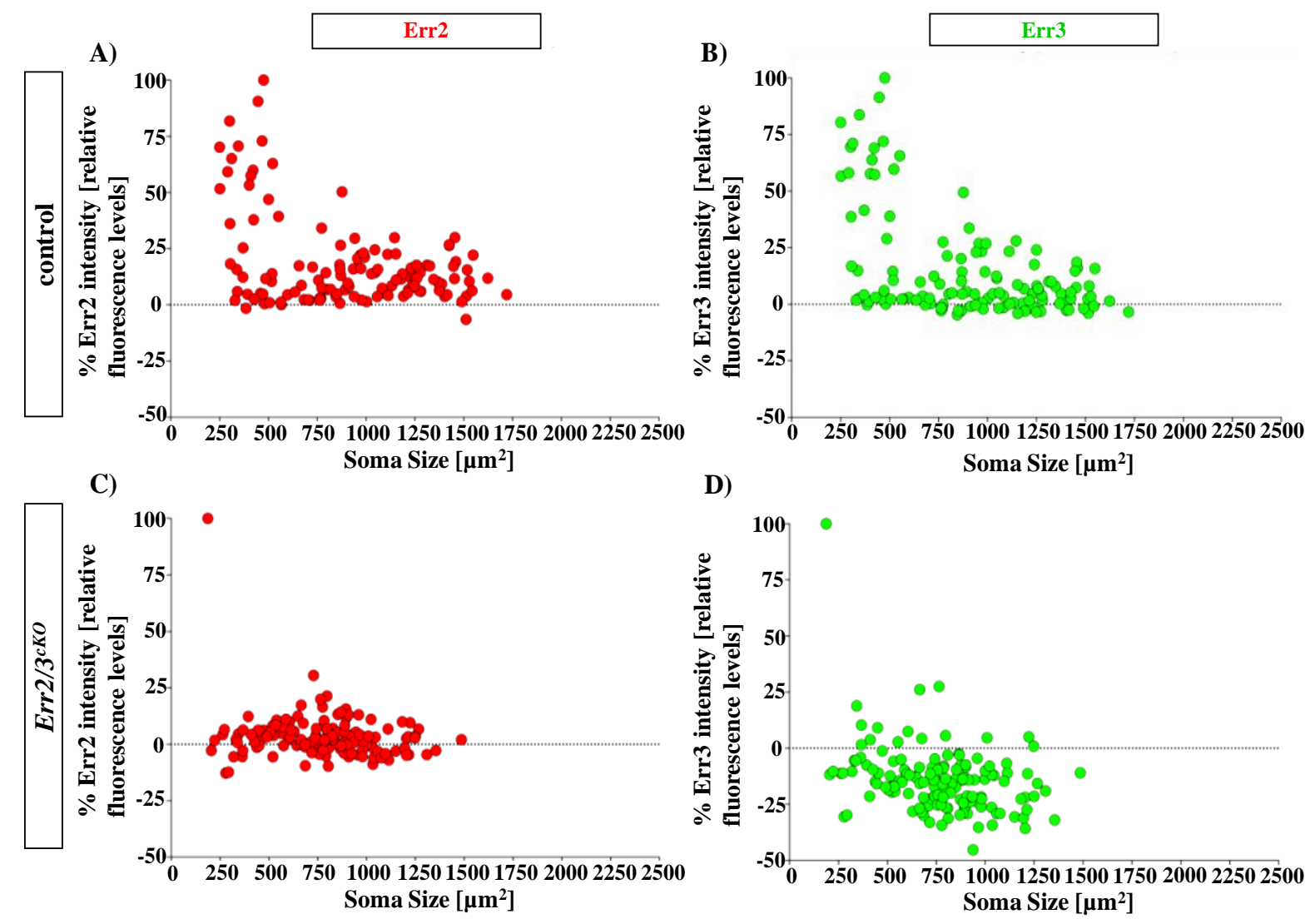

Figure 3.5 Lack of Err2 and Err3 relative expression levels in the putative $\gamma$-MNs from $E r r 2 / 3^{c K O}$ mice compared to control mice

(A-D) Scatter-plots of normalized Err2 and Err3 expression levels in control and Err2/3 ${ }^{c K O}$ mice where the $\mathrm{x}$-axis represents the soma size in $\mu \mathrm{m}^{2}$ and $\mathrm{y}$-axis represents normalized \% fluorescence intensity in relative fluorescence levels. (A, B) Control relatively small soma size MNs $\left(\sim 250 \mu \mathrm{m}^{2}\right.$ $500 \mu \mathrm{m}^{2}$ ) show a pattern with high expression levels of Err2 and Err3. (C, D) Whereas Err2/3 ${ }^{c K O}$ relatively small soma size MNs $\left(\sim 250 \mu \mathrm{m}^{2}-500 \mu \mathrm{m}^{2}\right)$ overall lack the expression of Err2 and Err3.

\subsubsection{Characterization of putative $\gamma$-MN connectivity in $E r r 2 / 3^{c K O}$ mice}

Previous studies have shown that $\gamma$-MNs lack direct innervation of Ia sensory afferents, whereas $\alpha$-MNs are directly innervated by Ia sensory afferents (Eccles et al., 1957b; Eccles et al., 1960; Friese et al., 2009). Thus, I analyzed whether the loss of Err2 and Err3 in MNs from Err $2 / 3^{c K O}$ mice leads to changes in Ia sensory innervation. The Ia sensory synaptic termini are enriched in vGlut1 (vesicular glutamate transporter 1) synaptic varicosities apposed to the $\alpha$-MNs somatic and dendritic membrane. vGlut1 is a transporter protein that incorporates glutamate, the primary excitatory neurotransmitter used in the nervous system. Similar to previous quantitative immunodetection studies, cryosections from mice injected with FG retrograde tracer were treated with specific anti-Err2, anti-NeuN and anti-vGlut1 primary antibodies and appropriate secondary antibodies and imaged using a confocal microscope. Subsequently, neural surfaces and synaptic varicosities were rendered 
and visualized using Imaris software (Bitplane AG) (Fig. 3.6A-E) (Materials \& Methods section 2.12.7).
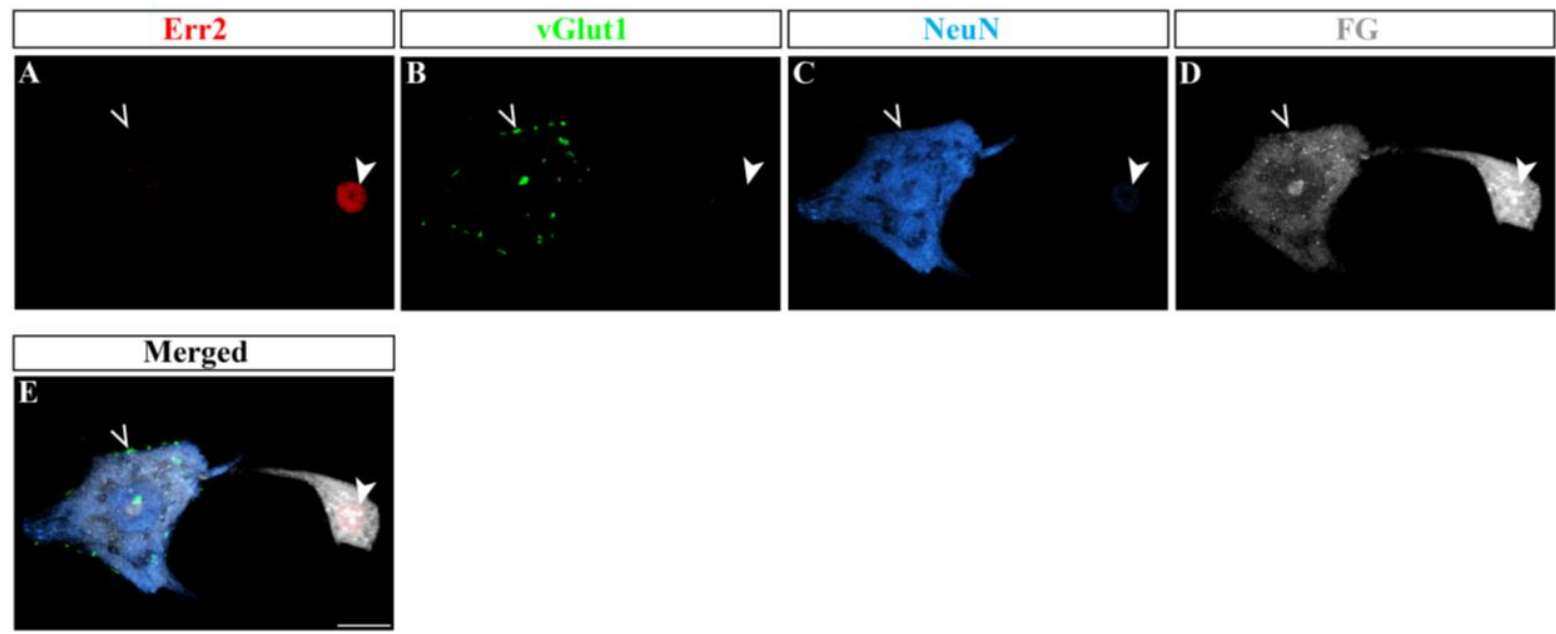

Figure 3.6 Visualization of Ia sensory synaptic termini enriched in vGlut ${ }^{+}$synaptic varicosities

(A-E) Rendered images using Imaris (Bitplane A.G.) were used to visualize vGlut1 synaptic varicosities on MN soma and proximal dendritic membrane. In control mice, a putative $\alpha$-MN has low expression levels of Err2, high expression levels of NeuN and low FG levels and a putative $\gamma$-MN with high expression levels of Err2, low expression levels of NeuN and high FG levels. A) Err2 ${ }^{+}$(red nuclei, solid arrow), B) vGlut1 ${ }^{+}$(green synaptic varicosities, hollow arrow), C) NeuN, D) FluoroGold (FG), E) Merged image of all channels. High number of vGlut1 synaptic varicosities (hollow arrow) on the putative $\alpha-\mathrm{MN}$ and essential lack of vGlut $1^{+}$synaptic varicosities on the putative $\gamma-\mathrm{MN}$. Scale bar represents $20 \mu \mathrm{m}$.

In control mice, relatively small soma size $\left(250 \mu \mathrm{m}^{2}-500 \mu \mathrm{m}^{2}\right)$ putative $\gamma$-MNs from control mice essentially lacked vGlut ${ }^{+}$synaptic varicosities (Fig. 3.7A-C, M). Compared to the putative $\gamma$-MNs from control mice, the putative $\gamma$-MNs from $E r r 2 / 3^{c K O}$ essentially lacked vGlut $1^{+}$varicosities and thus, were not significantly different from control mice (Fig. 3.7D-F, M). Relatively large soma size $\left(>600 \mu \mathrm{m}^{2}\right)$ putative $\alpha$-MNs had a high number of vGlut $1^{+}$ synaptic varicosities (Fig. 3.7G-I, M). Compared to the putative $\alpha$-MNs from control mice, the putative $\alpha$-MNs from Err2/3 ${ }^{c K O}$ mice also had a high number of vGlut $1^{+}$varicosities and were not significantly different from control mice (Fig. 3.7J-L, M). Therefore, the data showed that Ia afferent connectivity is not altered in Err $2 / 3^{c K O}$ mice (Fig. 3.7A-M). 

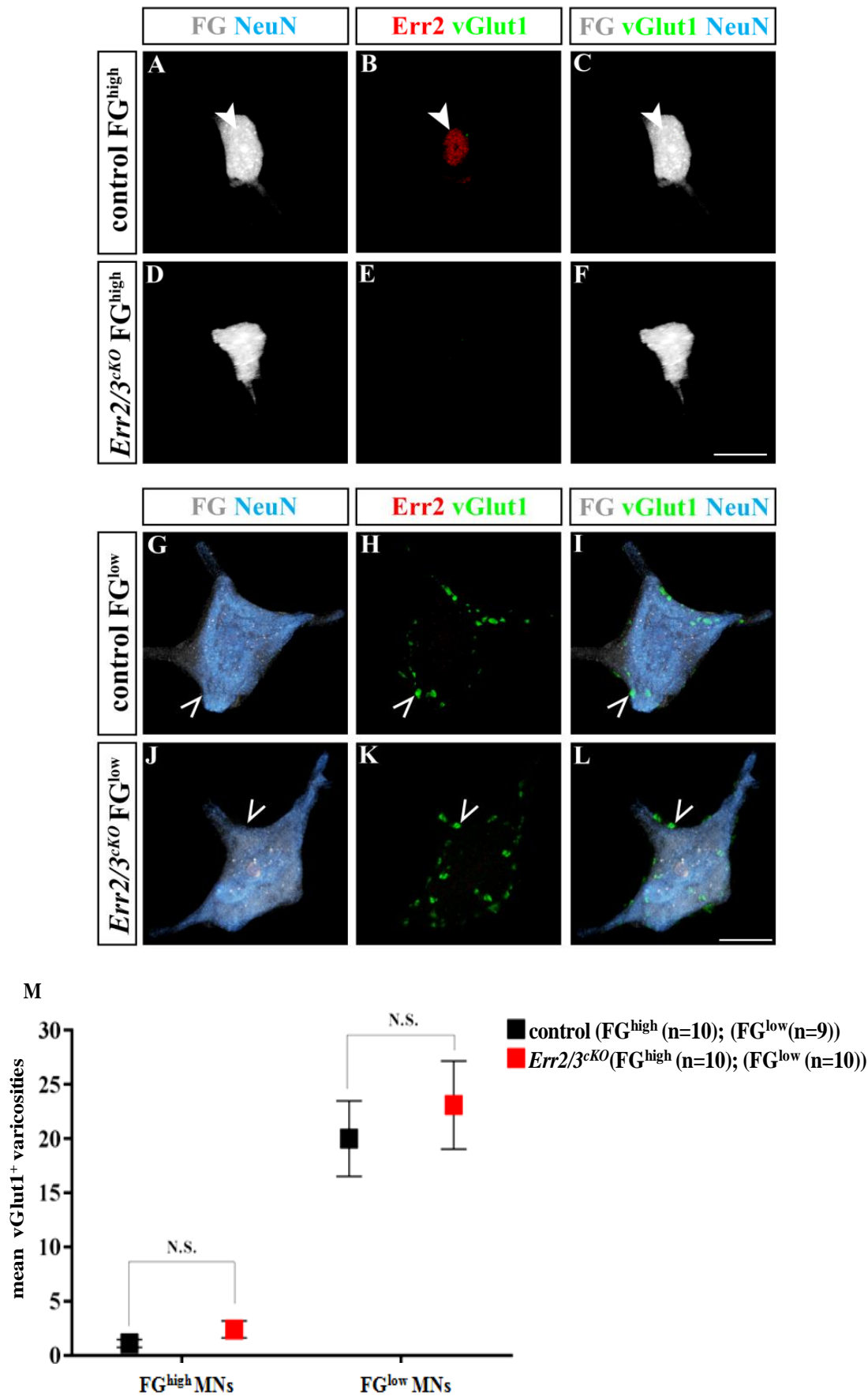

Figure 3.7 Ia sensory afferent connectivity is not altered in $\mathrm{Err} 2 / \mathrm{3}^{c K O}$ mice

(A-M) Representative rendered images using Imaris software. (A-C) A putative $\gamma-\mathrm{MN}$ from control mice that is $\mathrm{Err}^{+}$(solid arrow) and incorporates high levels of FG and lacks vGlut $1^{+}$synaptic varicosities. (D-F) A putative $\gamma$-MN from $E r r 2 / 3^{c K O}$ mice that is Err2 ${ }^{-}$and incoporates high levels of FG, lacks vGlut ${ }^{+}$synaptic varicosities. (G-I) A putative $\alpha-\mathrm{MN}$ from control mice that is Err2 ${ }^{-}$, has high levels of NeuN and incorporates low levels of FG, has a high number of vGlut $1^{+}$synaptic varicosities (green, hollow arrow). (J-L) putative $\alpha$-MN from $E r r 2 / 3^{c K O}$ mice that is Err2 $2^{-}$and has high levels of NeuN and incoporates low FG levels, also has a high number of vGlut ${ }^{+}$synaptic varicosities (hollow arrow). (M) Quantification of ${\text { vGlut } 1^{+} \text {synaptic varicosities comparing } \mathrm{FG}^{\text {high }}}$ control (putative $\gamma$-MNs) versus $\mathrm{FG}^{\text {high }} E r r 2 / 3^{\text {cKO }}$ (putative $\gamma$-MNs) and $\mathrm{FG}^{\text {low }}$ control (putative $\alpha$-MN) versus $\mathrm{FG}^{\text {low }}$ Err $2 / 3^{\text {cKO }}$ (putative $\alpha$-MNs). Control $\mathrm{FG}^{\text {high }}$ putative $\gamma$-MNs $(1.1 \pm 0.368, \mathrm{n}=10, \mathrm{~N}=6)$ showed no significant differences when compared to $E r r 2 / 3^{c K O} \mathrm{FG}^{\text {high }}$ putative $\gamma$-MNs: $2.4 \pm 0.781$, $\mathrm{n}=10, \mathrm{~N}=4 ; p=0.190$ ). And control $\mathrm{FG}^{\text {low }}$ putative $\alpha-\mathrm{MNs}$ (mean \pm S.E.M. $\mathrm{n}=$ neurons, $\mathrm{N}=$ animals; 20 
$\pm 3.48, \mathrm{n}=9, \mathrm{~N}=6$ ) showed no significant differences when compared to $E r r 2 / 3^{c K O} \mathrm{FG}^{\text {low }}$ putative $\alpha$ MNs $(23.1 \pm 4.06, \mathrm{n}=10, \mathrm{~N}=4 ; p=0.343)$. Scale bar represents $20 \mu \mathrm{m}$.

\subsection{Characterization of MN biophysical properties in $E r r 2 / 3^{c K O}$ mice}

Next, studies were designed to test the effects of the loss of Err2 and Err3 in the putative $\gamma$-MN biophysical properties from Err $2 / 3^{c K O}$ mice. Control (Chat::Tomato) and Err $2 / 3^{c K O}$ were intraperitoneally injected with FG because I consistently observed that relatively small soma size MNs (putative $\gamma$-MNs) incorporated high levels of FG in their soma (Fig. 3.8). Whereas relatively large soma size MNs (putative $\alpha$-MNs) incorporated low levels of FG in their soma (Fig. 3.8). Thus, I used the levels of FG and relative soma size to identify and perform whole cell patch-clamp recordings in putative $\alpha$ - versus $\gamma$-MNs from control and $E r r 2 / 3^{c K O}$ mice at three weeks of age (Fig. 3.8).

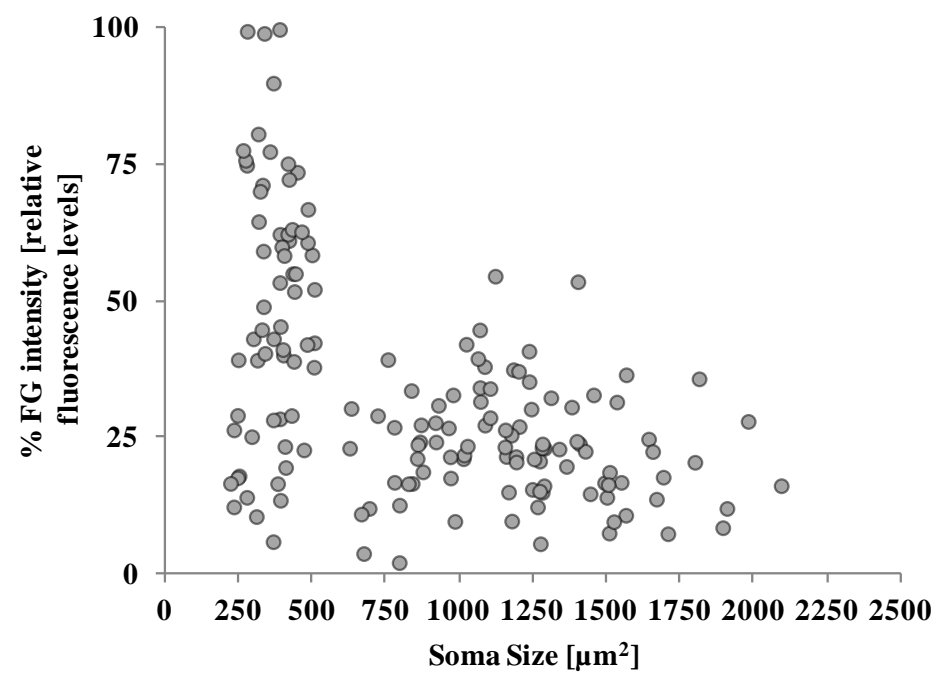

Figure 3.8 Comparison of FG intensity and soma size to target $\gamma$-MNs in P21 W.T. mice for recordings

Scatter-plots of normalized FG incoporation in control mice where the $\mathrm{x}$-axis represents the soma size in $\mu \mathrm{m}^{2}$ and $\mathrm{y}$-axis represents normalized \% fluorescence intensity in relative fluorescence levels. FG combined with soma size enabled the targeting of whole cell patch-clamp recordings in putative $\alpha-$ versus $\gamma$-MNs.

Several classical biophysical properties were analyzed after recording from putative $\alpha$ - versus $\gamma$-MNs. Some of these included the rheobase, the input resistance, the capacitance, the F-I curve, the AHP amplitude, the AHP half-width and the AHP half-decay times (Materials \& Methods section 2.14.5) (Muramoto et al., 1996; Miles et al., 2004, 2005; Manuel et al., 2009; Nakanishi and Whelin, 2010; Mitra and Brownstone, 2012; Hadzipasic et al., 2014; Müller et al., 2014). Furthermore, the repetitive firing patterns of action potentials due to the square current pulse to MNs during the $F-I$ curve protocol enabled the 
opportunity to look more in-depth at MN firing behavior (Manuel et al., 2009; Hadzipasic et al., 2014).

Previous studies in cat found that biophysical properties including low firing threshold and a high discharge rate were characteristic of $\gamma$-MNs (Kemm and Westbury, 1978). Thus, I wished to compare properties like rheobase, firing frequency and gain between $\alpha-$ and $\gamma-\mathrm{MN}$ and distinguish them reliably. When the rheobase was plotted versus the mean firing frequency or the gain on a scatter-plot, the clustered patterns of MNs based on these properties allowed me to compare $\alpha$-MN versus $\gamma$-MNs biophysical signatures. Thus, I assessed the effects of loss of Err2 and Err3 in putative $\gamma$-MN biophysical signature from Err $2 / 3^{c K O}$ mice in comparison to control mice. When comparing the rheobase versus mean firing frequency or gain by entire duration of depolarizing current pulse $(1 \mathrm{~s})$, first interspike interval (instantaneous), or $0.5 \mathrm{~s}$ (steady-state) (Materials \& Methods section 2.14.5).

The data for the mouse whole cell patch-clamp studies are summarized in Table 8.1 of Appendix 2. First, I tested whether the levels of FG were sufficient to distinguish the difference in $\alpha$-versus $\gamma$-MN biophysical properties in control mice. The comparison of mean rheobase, mean firing frequency and mean gain showed that the MNs that incorporated high levels of FG (putative $\gamma$-MNs) showed lower mean rheobase (Fig. 3.9A), higher mean firing frequency (Fig. 3.9B) and higher mean gain (Fig. 3.9C), when compared to the MNs that incorporated low levels of FG (putative $\alpha-\mathrm{MNs}$ ) (Fig. 3.9A-C). Thus, the electrophysiological data suggest FG levels and relative soma size can be employed as a ground for distinguishing MN subtypes for the recordings. Thus, $\mathrm{FG}^{\text {high }}$ (putative $\gamma$-MNs) and $\mathrm{FG}^{\text {low }}$ (putative $\alpha-\mathrm{MNs}$ ) from control and $E r r 2 / 3^{c K O}$ mouse spinal cord slices were subsequently recorded.

\subsubsection{Characterization of putative $\alpha$ - and $\gamma$-MNs biophysical properties in control versus $\operatorname{Err} 2 / 3^{c K O}$ mice}

I next compared the biophysical signatures of putative $\alpha$ - and $\gamma$-MNs from control and Err $2 / 3^{c K O}$ mice. The mean rheobase for the putative $\gamma$-MNs from Err $2 / 3^{c K O}$ mice was significantly higher than the mean rheobase for the putative $\gamma$-MNs from the control mice (Fig. 3.9A). The mean firing frequency for the putative $\gamma$-MNs from $\operatorname{Err} 2 / 3^{c K O}$ mice was significantly lower than the mean firing frequency of the putative $\gamma$-MNs from control mice (Fig. 3.9B). And the mean gain for the putative $\gamma$-MNs from $\operatorname{Err} 2 / 3^{c K O}$ was significantly lower than the mean gain for the putative $\gamma$-MNs from control mice (Fig. 3.9C). When comparing control versus $E r r 2 / 3^{c K O}$ putative $\alpha$-MNs, there were no significant differences in the mean rheobase (Fig. 3.9A), mean firing frequency (Fig. 3.9B) or mean gain (Fig. 3.9C). 

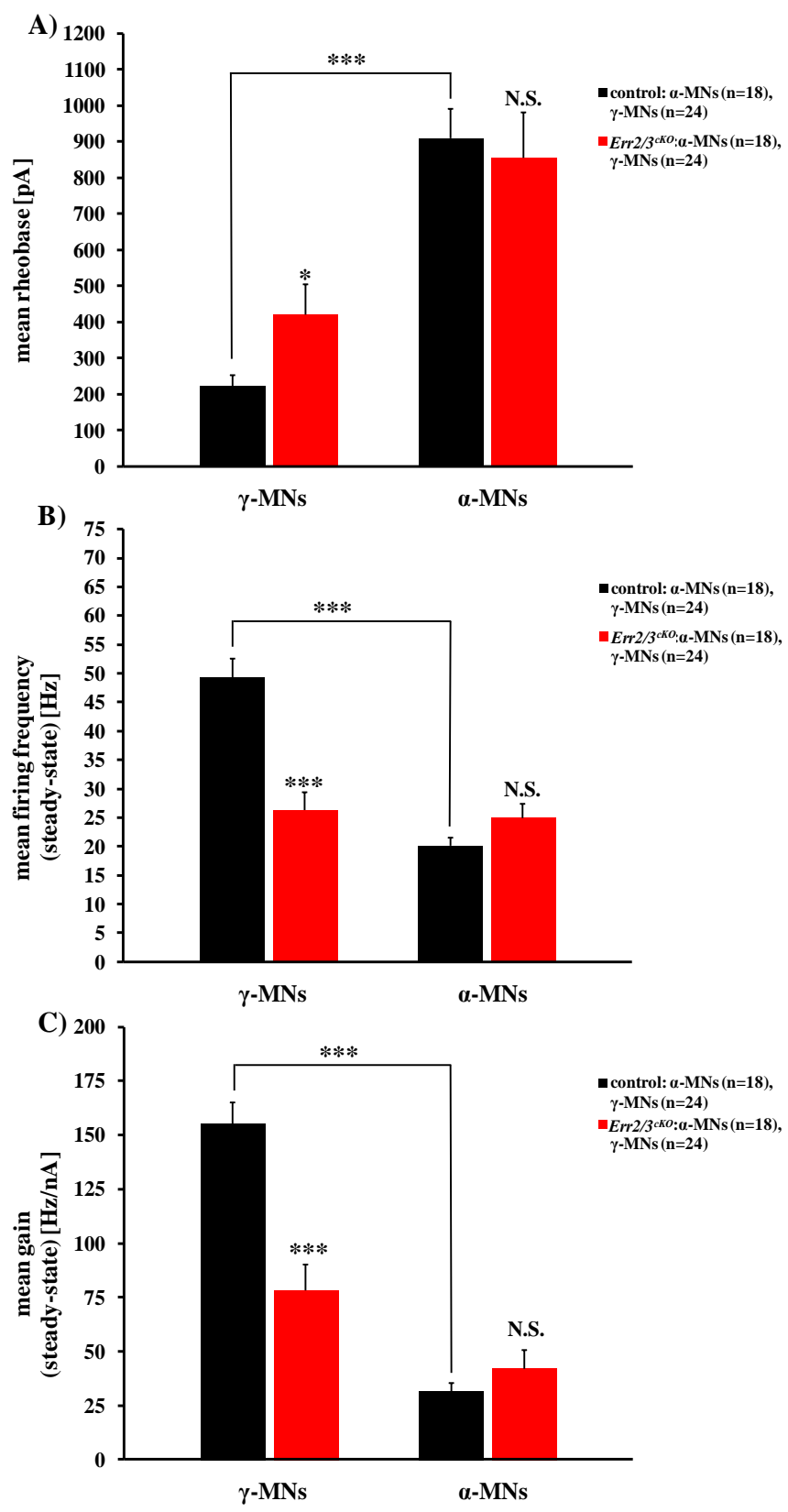

Figure 3.9 The loss of Err2 and Err3 in the putative $\gamma$-MNs from $E r r 2 / 3^{c K O}$ mice results in shifted biophysical properties towards putative $\alpha$-MNs

(A-C) Bar graph showing comparison of biophysical properties between control putative $\gamma$-MNs versus $E r r 2 / 3^{c K O}$ putative $\gamma$-MNs, control putative $\alpha$-MNs versus $E r r 2 / 3^{c K O}$ putative $\alpha$-MNs and control putative $\gamma$-MNs versus control putative $\alpha$-MNs. (A) The putative $\gamma$-MNs from $E r r 2 / 3^{c K O}$ had a much higher mean rheobase than the putative $\gamma$-MNs from control mice. While there were no differences in mean rheobase between putative $\alpha$-MNs from $E r r 2 / 3^{c K O}$ and control mice. B) The putative $\gamma$-MNs from $E r r 2 / 3^{c K O}$ mice had a much lower mean firing frequency (steady-state) than the putative $\gamma$-MNs from control mice. While no differences in mean firing frequency (steady-state) was observed between putative $\alpha$-MNs from $E r r 2 / 3^{c K O}$ and control mice. C) The putative $\gamma$-MNs from $E r r 2 / 3^{c K O}$ mice had a much lower mean gain (steady-state) than the putative $\gamma$-MNs from control mice. And no differences in mean gain (steady-state) was observed in putative $\alpha-\mathrm{MNs}$ from $E r r 2 / 3^{c K O}$ and control mice. Therefore, Err $2 / 3^{c K O}$ putative $\gamma$-MNs show properties that are more like putative $\alpha$-MNs. Comparison of biophysical properties of putative $\gamma$ MNs versus $\alpha$-MNs from control mice: (A) The mean rheobase for putative $\gamma$-MNs was significantly lower than the mean rheobase for putative $\alpha$-MNs. (B) While the mean firing frequency (steady-state) and (C) the mean gain (steady-state) were significantly higher for putative $\gamma$-MNs when compared to putative 
$\alpha$-MNs. Thus, putative $\alpha-\mathrm{MN}$ properties are distinct from putative $\gamma$-MN properties in MNs from control mice.

The F-I curve protocol of incremental square current pulses to MNs showed significantly different voltage responses when comparing the putative $\gamma$-MNs from control mice versus the putative $\gamma$-MNs from $E r r 2 / 3^{c K O}$ mice (Fig. 3.10A-F) (Materials \& Methods section 2.14.5).

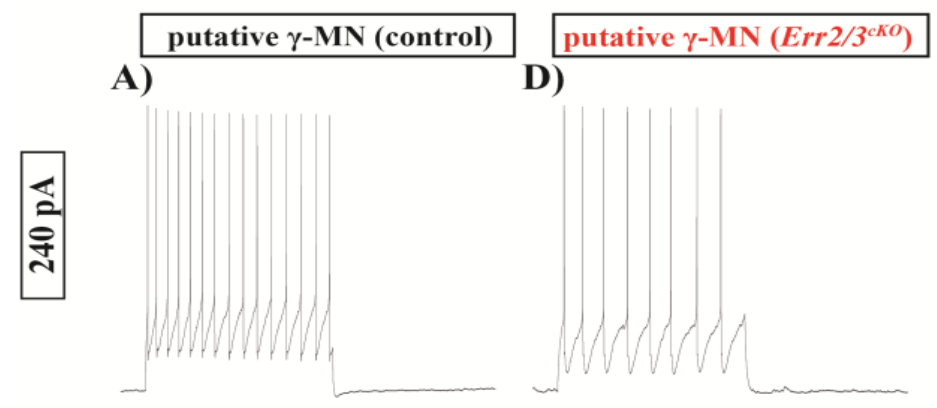

B)

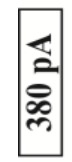

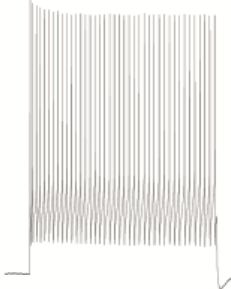

C)

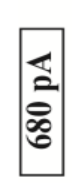

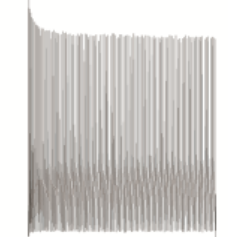

E)

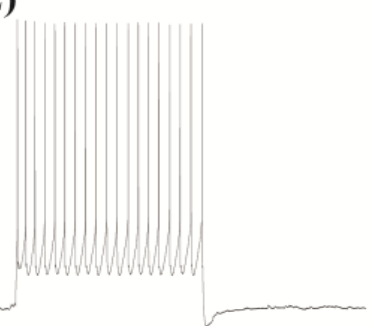

F)

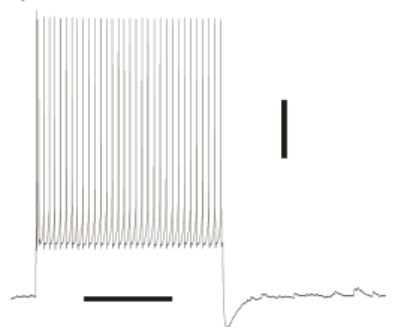

Figure 3.10 A putative $\gamma$-MN from $E r r 2 / 3^{c K O}$ mice loses its biophysical signature and shifts towards $\alpha$-MN biophysical properties

(A-F) Representative voltage response traces in a putative $\gamma$-MN (control and $E r r 2 / 3^{c K O}$ ) subjected to increments of $20 \mathrm{pA}, 1000 \mathrm{~ms}$ square current pulses during $F$-I curve square current pulse protocol. (A, D) a $240 \mathrm{pA}, 1000 \mathrm{~ms}$ square current pulse to a putative $\gamma$-MN from control mice leads to a higher firing frequency compared to a putative $\gamma$-MN from $E r r 2 / 3^{c K O}$ mice. (B, E) This pattern continues when $380 \mathrm{pA}, 1000 \mathrm{~ms}$ square current pulse is applied to the MNs. (C, F) The firing frequency of a putative $\gamma$-MN from control mice further increases when a $680 \mathrm{pA}, 1000 \mathrm{~ms}$ square current pulse is applied to the $\mathrm{MN}$, however, the putative $\gamma$-MN from $\operatorname{Err} 2 / 3^{c K O}$ mice shows a significantly lower increase in firing frequency. Scale bars represent $0.5 \mathrm{~s}$ duration of the square current pulse and $20 \mathrm{mV}$ of the voltage-response due to the current pulse.

For the whole cell patch-clamp recording data, I plotted the rheobase versus mean firing frequency or gain for putative $\gamma$-MNs from control and Err2/3 ${ }^{c K O}$ mice (Fig. 3.11A, B). When compared to control, the putative $\gamma$-MNs from $E r r 2 / 3^{c K O}$ mice showed a clear shift of 
MN biophysical properties towards higher rheobase, lower mean firing frequency and lower gain (Fig. 3.11A, B). Taken together, these data suggest that in Err $2 / 3^{c K O}$ mice, putative $\gamma$ MNs lose their biophysical signature and shift towards that of $\alpha$-MNs.

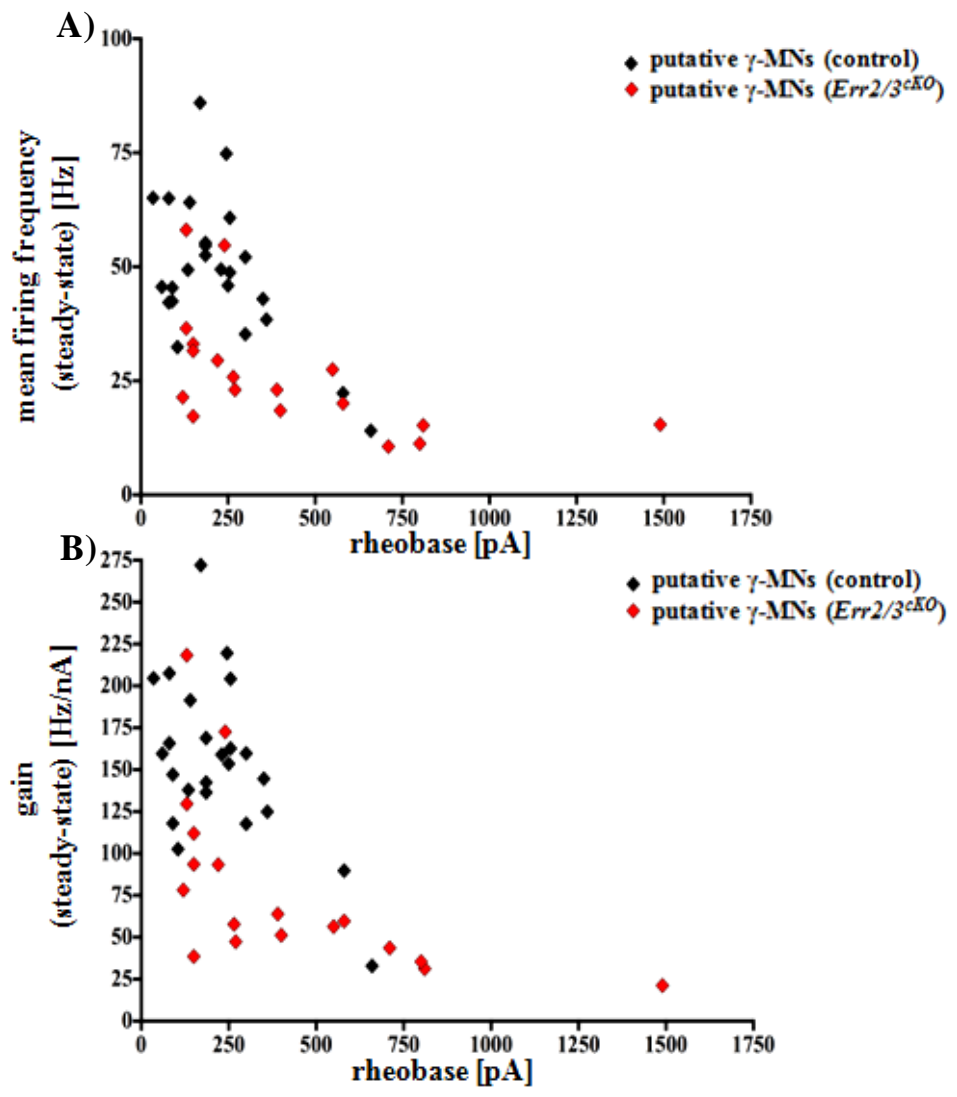

Figure 3.11 The putative $\gamma$-MNs from $E r r 2 / 3^{c K O}$ lose their biophysical signature and shift their biophysical properties towards $\alpha$-MNs

(A) Scatter-plot showing rheobase (pA) (x-axis) versus mean firing frequency (Hz) (steady-state) (yaxis) in putative $\gamma$-MNs from control versus $E r r 2 / 3^{c K O}$ mice. Compared to control, the putative $\gamma$-MNs from $E r r 2 / 3^{c K O}$ mice show a shift in the rheobase (higher) and the mean firing frequency (lower). (B) Scatter-plot showing rheobase (pA) (x-axis) versus gain (Hz/nA) (steady-state) (y-axis) for putative $\gamma$ MNs from control and $E r r 2 / 3^{c K O}$. The putative $\gamma$-MNs in $E r r 2 / 3^{c K O}$ mice show a shift in rheobase (higher) and gain (lower) when compared to the putative $\gamma$-MNs from control mice.

Moreover, there was not a shift in the clustering of putative $\alpha-\mathrm{MN}$ properties from Err $2 / 3^{c K O}$ mice when compared to the putative $\alpha-\mathrm{MNs}$ in control mice (Fig. 3.12A, B). In summary, the lack of Err2 and Err3 expression in the putative $\gamma$-MNs from Err2/3 ${ }^{c K O}$ mice leads to significant changes in the biophysical properties when compared to the putative $\gamma$ MNs from control mice. While no significant changes in the biophysical properties were observed in the putative $\alpha$-MNs from $E r r 2 / 3^{c K O}$ mice compared to the putative $\alpha$-MNs from control mice. 


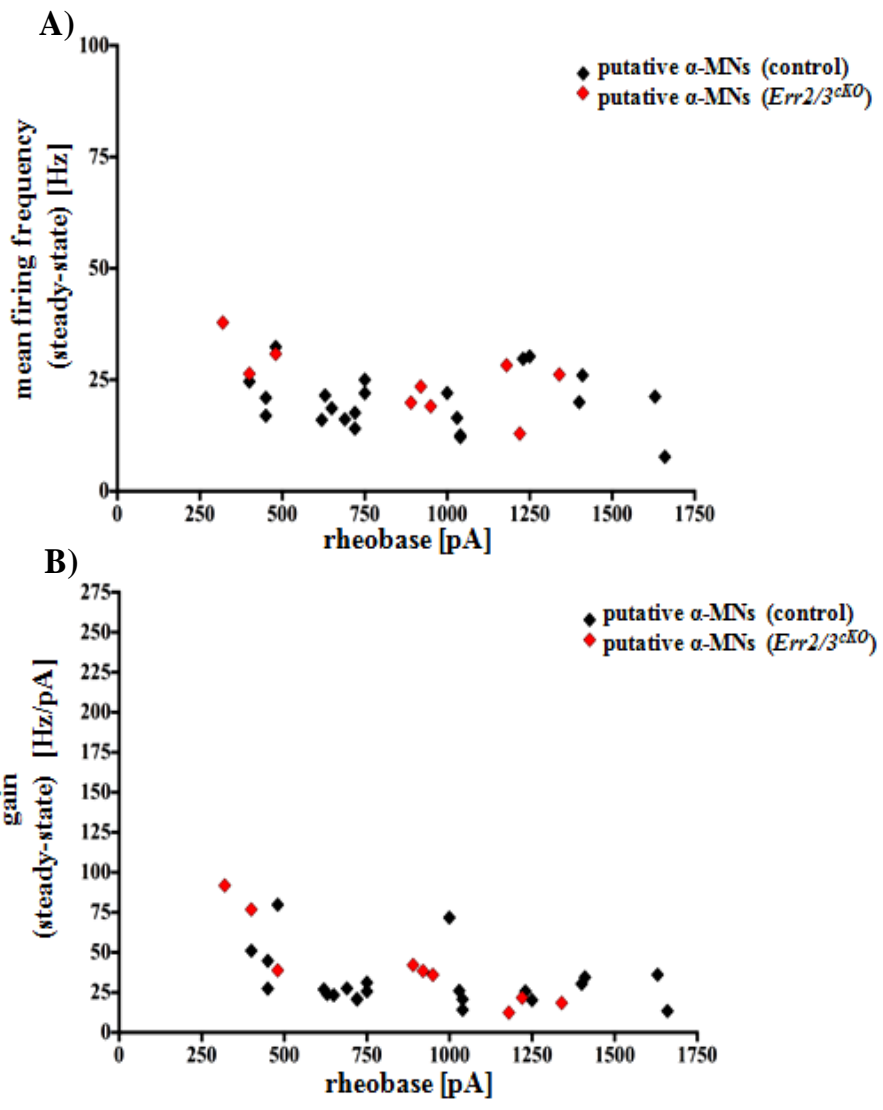

Figure 3.12 The putative $\alpha$-MNs from $E r r 2 / 3^{c K O}$ do not shift their biophysical properties

(A, B) Scatter-plots showing rheobase (pA) (x-axis) versus firing frequency (Hz) (steady-state) (yaxis) or gain (Hz/nA) (steady-state) (y-axis) in putative $\alpha$-MNs from control versus $E r r 2 / 3^{c K O}$ mice. There were no shifts in putative $\alpha$-MNs pattern of biophysical properties in $E r r 2 / 3^{c K O}$ when compared to the putative $\alpha-\mathrm{MNs}$ from control mice.

\subsection{Characterization of gait patterns and precision movements during locomotion in Err2/3 ${ }^{c K O}$ mice}

Studies in cat have shown that proprioceptive feedback information from the muscle spindle (MS) and the Golgi tendon organ (GTO) is involved in the proper coordination of locomotion (Lam and Pearson, 2001; Grillner et al., 1978; Hiebert et al., 1996; Duysens et al., 1980; Mcvea 2005). Similarly, studies in mice showed that normal locomotion depends on the proprioceptive feedback from MSs (Akay et al., 2014). Herein, mice with mutations in Egr3 (early growth response 3), were not able to develop MSs resulting in abnormal gait in mice (Tourtellotte et al., 1998, 2001). These locomotion defects included altered timing of the ankle flexor muscle action (Akay et al., 2014). However, the precise role of $\gamma$-MNs in determining motor behavior remains to be addressed in vivo. Hence, I hypothesized that the lack of Err2 and Err3 and the resulting shift towards $\alpha$-MN properties in the putative $\gamma$-MNs of $E r r 2 / 3^{c K O}$ mice could disrupt MS function and therefore result in gait and precision 
movement deficits. Thus, I tested whether $E r r 2 / 3^{c K O}$ mice would display abnormal gait patterns when running on a treadmill or performing more skilled tasks, such as walking across a horizontal ladder and beam.

\subsubsection{Characterization of gait in $E r r 2 / 3^{c K O}$ mice using the DigiGait ${ }^{\mathrm{TM}}$ treadmill task $^{\mathrm{N}}$}

To assess gait deficits, I used the DigiGait ${ }^{\mathrm{TM}}$ imaging system (Mouse Specifics Inc., Framingham, MA U.S.A.), which uses a motorized treadmill installed with a transparent belt and a high-speed digital video camera to record animal locomotion from below (Materials \& Methods section 2.11.1) (Amende et al., 2005, Müller et al., 2014; Yuengert et al., 2015). Control (Chat-Cre) littermates and $\mathrm{Err} 2 / 3^{c K O}$ littermates age-matched at 8-weeks of age were tested at six different treadmill belt speeds: $10 \mathrm{~cm} / \mathrm{s}, 15 \mathrm{~cm} / \mathrm{s}, 20 \mathrm{~cm} / \mathrm{s}, 25 \mathrm{~cm} / \mathrm{s}, 30 \mathrm{~cm} / \mathrm{s}, 35$ $\mathrm{cm} / \mathrm{s}$. Various gait parameters were analyzed by the DigiGait ${ }^{\mathrm{TM}}$ imaging system including stride, stance, swing, braking, and propulsion (Fig. 2.4 in Materials \& Methods section 2.11.1).

The Digigait ${ }^{\mathrm{TM}}$ analysis produced a large dataset with several variables for a small number of control and $E r r 2 / 3^{c K O}$ mice. Thus, a method of multivariate analysis was required to understand and interpret the data. Thus, the Orthogonal Signal Correction Partial Least Squares (OSC-PLS) method was performed to analyze the dataset (Materials \& Methods section 2.11.1.1). This method uses Principal Component Analysis (PCA) and Multiple Regression to rank or predict variables that are most important for explaining the variation in gait between control and $\operatorname{Err} 2 / 3^{c K O}$ mice.

The overall difference in gait between control and $E r r 2 / 3^{c K O}$ mice was determined while considering all gait metrics initially. Scatter-plots with polygon graphs demonstrated the ability of the validated model to separate control and Err2/3 ${ }^{c K O}$ mouse groups (Fig. 3.13A, B). An optimized model with 2 components or dimensions plotted against each other was able to describe $>25 \%$ of the variance in the predictors of the mouse forelimb and hindlimb gait (Fig. 3.13A, B) (Table 2.9 Materials \& Methods section 2.11.1.1). The separation between control and $E r r 2 / 3^{c K O}$ mice is visualized by a scatter-plot with polygons added to visualize the two cluster groups. Based on PCA- 1 alone, control and Err $2 / 3^{c K O}$ mice were clustered into two groups for the forelimbs and the hindlimbs, thus, this component by itself was sufficient to understand the model. Overall, the data analysis suggests that control and $E r r 2 / 3^{c K O}$ mice show different gait patterns. 

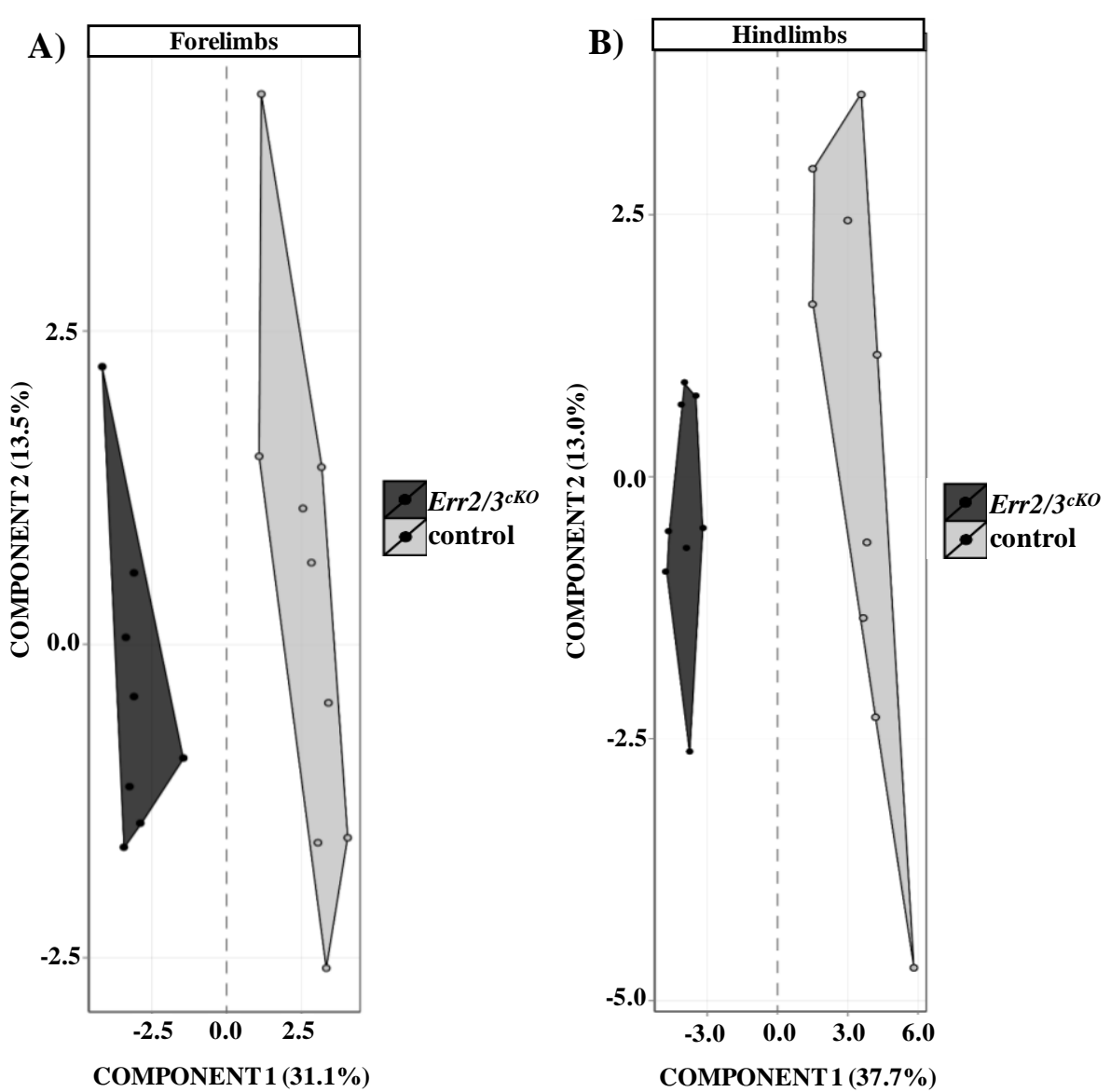

Figure 3.13 $\mathrm{Err} 2 / \mathrm{3}^{\mathrm{cKO}}$ mice have a different gait pattern in the forelimbs and the hindlimbs when compared to control mice

(A) Scatter-plots with polygon graphs depicting the maximum variance between-group (PCA-1, $\mathrm{x}$ axis) and the maximum variance within-group (PCA-2, y-axis) in the forelimbs for control versus Err $2 / 3^{c K O}$ groups. Each individual dot (score) in the scatter-plot represents a single mouse projection across 2 partial least square (PLS) regression components of the model. The percentage of variance explained by each component is shown as percent of the total variance marked on the axes for the model components. Principle Component 1 (PCA-1) explains the maximum between-group variance of $31.1 \%$ and is sufficient to separate mice into two genotypes, while Principle Component 2 (PCA-2) explains a maximum within-group variance of $13.5 \%$ in forelimbs of control and $E r r 2 / 3^{c K O}$. Polygon of $E r r 2 / 3^{c K O}$ (dark gray) shows a separate cluster of mice from control (light gray) polygon. (B) Similar to the forelimbs, the hindlimbs PCA-1 explains a maximum between-group variance of $37.7 \%$ and PCA-2 explains a maximum within-group variance of $13.0 \%$. Furthermore, PCA-1 is also sufficient to separate the control versus $\operatorname{Err} 2 / 3^{c K O}$ mice. Thus, control versus $\operatorname{Err} 2 / 3^{c K O}$ mice comparison shows different gait characteristics in the forelimbs and the hindlimbs at $25 \mathrm{~cm} / \mathrm{s}$ treadmill speed.

The variables (gait parameters) important in determining the differences in gait pattern between control and Err $2 / 3^{c K O}$ mice were analyzed (Table 2.9 in Materials \& Methods section 2.11.1.1). For example, at an arbitrary threshold, the most predictive gait parameters can be seen on the bar graph (Fig. 3.14). Err $2 / 3^{c K O}$ mice display reduced stanceswing ratio, thus, show an altered gait phase. $\operatorname{Err} 2 / 3^{c K O}$ mice show reduced propulsion velocities like max. DA/DT indicating shifts in force. Err $2 / 3^{c K O}$ mice show reduced stance 
width, which suggests shifts in posture. Moreover, Err2/3 $3^{c K O}$ mice also show increased paw angle variability, which points to shifts in stride during locomotion.

For all speeds, several gait parameters had a potent predictive capacity for distinguishing control versus Err $2 / 3^{c K O}$ mice in the forelimbs: absolute axis distance $(\mathrm{cm})$, stance width (cm), stride frequency (strides/s), amongst others (Table 9.1 in Appendix 3). Likewise, for the hindlimbs, several gait parameters predicted the genotypes powerfully between the two groups at all speeds: max DA/DT, paw area $\left(\mathrm{cm}^{2}\right)$, propulsion (ms), amongst other (Table 9.2 in Appendix 3).

Therefore, the changes seen in Err $2 / 3^{c K O}$ mice suggest that normally functioning $\gamma$ MNs are required for precise gait patterns during locomotion.

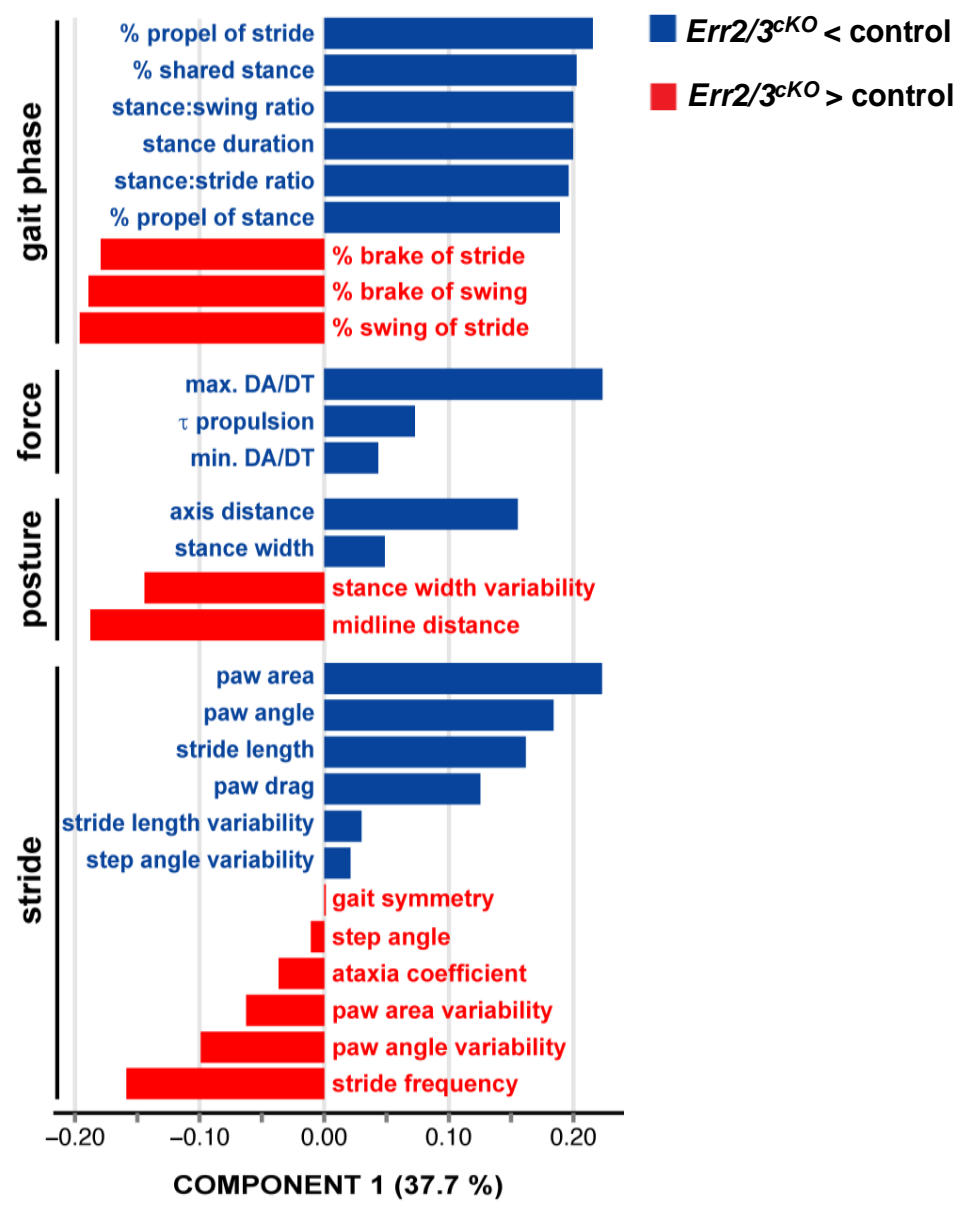

Figure 3.14 Specific gait variables predicted in distinguishing control versus $E r r 2 / 3^{c K O}$ mouse gait patterns

Color-coded bar graphs showing loadings for variables (gait parameters) that were important in distinguishing control versus Err2/3 ${ }^{c K O}$ mice gait patterns. Blue bars indicate mean loading of $E r r 2 / 3^{c K O}<$ mean loading of the control group. While red bars indicate mean loading of $E r r 2 / 3^{c K O}>$ mean loading of the control group. The sign of the bar (blue or red) indicates how the variable influences the direction of the phenotype of the animal. Thus, the highest blue or red bar representing loadings for gait parameters are ranked as the most predictive in determining the gait pattern of segregating control versus $E r r 2 / 3^{c K O}$ mice, which can be determined by the threshold 
value (0.20-(-0.20) in arbitrary units) on the $\mathrm{x}$-axis. These gait parameters can be grouped based on the relation to gait phase, force, posture and stride.

\subsubsection{The horizontal ladder task}

Previous studies have shown that Egr3-deficient mice lacking MSs exhibit abnormal gait patterns when navigating a horizontal ladder (Akay et al., 2014, Takeoka et al., 2014). Thus, the next set of experiments tested the precision movements in Err2/3 ${ }^{c K O}$ mice. Eight weeks-old age-matched control and $\operatorname{Err} 2 / 3^{c K O}$ mice were trained and tested to walk across a horizontal ladder while being recorded with a camera from below (Materials \& Methods section 2.11.2). The hind paws were scored as hits or misses (Fig. 3.15A). Analysis of the videos from the experiments showed that $E r r 2 / 3^{c K O}$ mice (mean misses \pm S.E.M., $\mathrm{N}=$ animals, $p=p$-value; $6.48 \pm 2.06, \mathrm{~N}=5, p=1.26 \mathrm{e}^{-6}$ ) committed more misses by a factor of $>6$ when compared to the control mice $(1.0 \pm 0.55, \mathrm{~N}=5)$ (Fig. 3.15B) (Materials \& Methods section 2.11.2 for "Miss Index" details). Thus, the data suggest that $E r r 2 / 3^{c K O}$ mice fail to place their hind paws precisely on the rungs during the horizontal ladder task due to impaired muscle proprioception.

A)

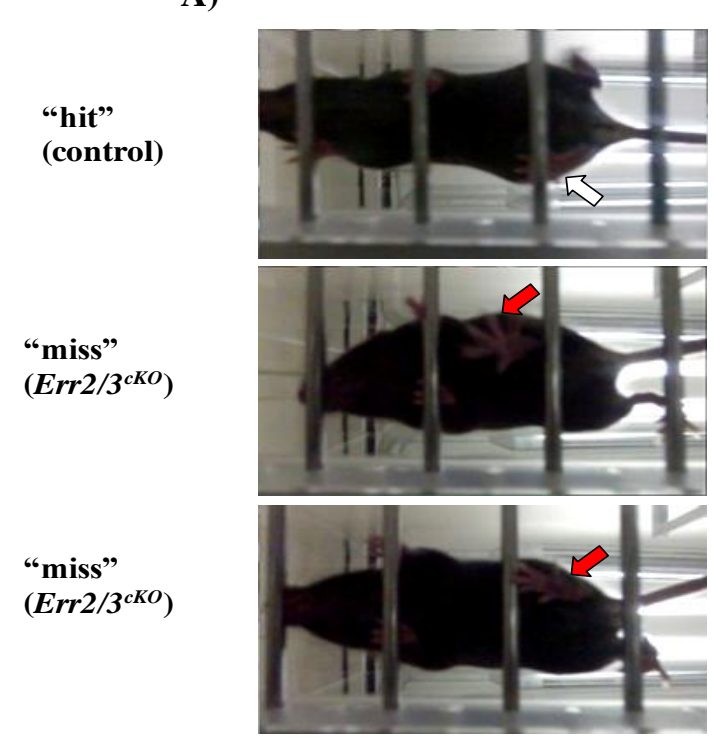

B)

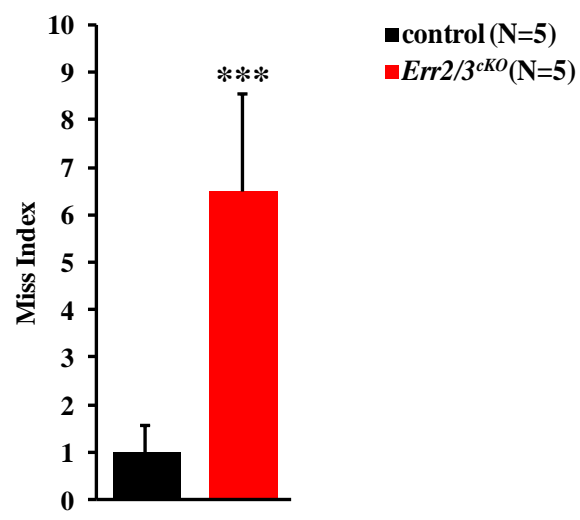

Figure 3.15 $\mathrm{Err} 2 / 3^{c K O}$ mice show deficits in precision movements when walking across a horizontal ladder

(A) Images from videos captured from below the mice as they traversed the horizontal ladder. The task was scored using specific criteria: 1) "hit" of the hindlimbs is scored when the mouse had a stable paw position in the middle of the ladder rung (white arrow), 2) "miss" is scored when the hind paw dropped through the rung (red arrow), 3) "miss" is scored when the mouse failed to locate the rung (red arrow).

(B) Bar graph showing the quantification of the hindlimb stepping errors for mice when walking across a horizontal ladder. $\operatorname{Err} 2 / 3^{c K O}$ mice committed more misses by a factor of $>6$-times when compared to control mice. 


\subsubsection{The horizontal beam task}

To further assess fine motor skills during locomotion, $\operatorname{Err} 2 / 3^{c K O}$ mice were challenged on a horizontal beam task (Bourane et al., 2015). Control and Err2/3 ${ }^{c K O}$ mice age-matched at 10 months-old were placed onto flat beams of $20 \mathrm{~mm}, 25 \mathrm{~mm}$ or $30 \mathrm{~mm}$ in width (Materials \& Methods section 2.11.3). The mice were recorded with a camera from the side as they performed the task. The hind paws were scored as hits or misses (Fig. 3.16A). Data analysis of the videos showed that $E r r 2 / 3^{c K O}$ mice (mean miss \pm S.E.M., $\mathrm{N}=$ animals, $p=p$-value; 22.09 $\pm 1.75, \mathrm{~N}=4, p=0.01)$ committed more misses by a factor of $>21$ when compared to control mice $(1.0 \pm 0.17, \mathrm{~N}=4)$ on the $30 \mathrm{~mm}$ beam width (Fig. 3.16B) (Materials \& Methods section 2.11.3 for "Miss Index" details). Similarly, data analysis showed that $\operatorname{Err} 2 / 3^{c K O}$ mice (50.36 \pm 2.20, $\mathrm{N}=4, p=5.2 \mathrm{e}^{-3}$ ) committed more misses by a factor of $>49$ when compared to control mice $(1.0 \pm 0.125, \mathrm{~N}=4)$ on $25 \mathrm{~mm}$ wide beam (Fig. 3.16B). Finally, on the $20 \mathrm{~mm}$ wide beam, $\operatorname{Err} 2 / 3^{c K O}$ mice $\left(63.50 \pm 3.49, \mathrm{~N}=4, p=9.0 \mathrm{e}^{-3}\right)$ committed more misses by a factor of $>62$ when compared to control mice $(1.0 \pm 0.125, \mathrm{~N}=4)$ (Fig. 3.16B). Thus, data indicate that Err $2 / 3^{c K O}$ mice show diminished precision movement skills which further decreased with the difficulty of the task.

A)

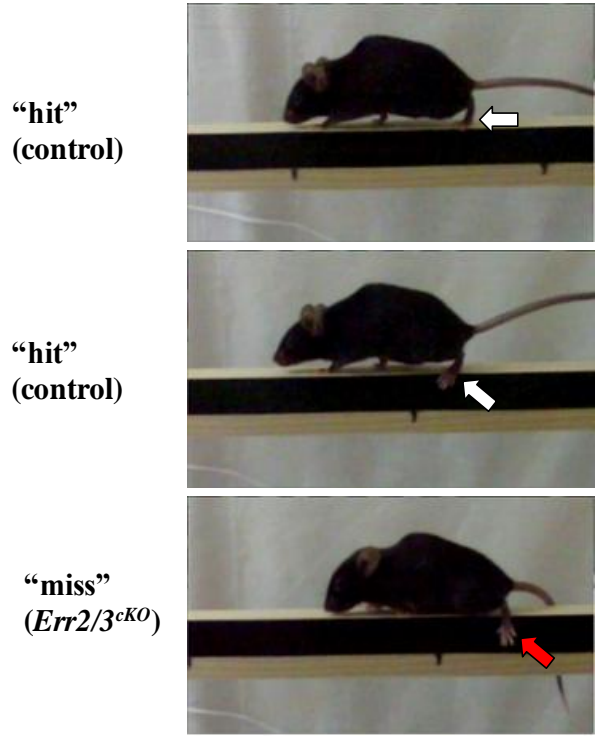

B)

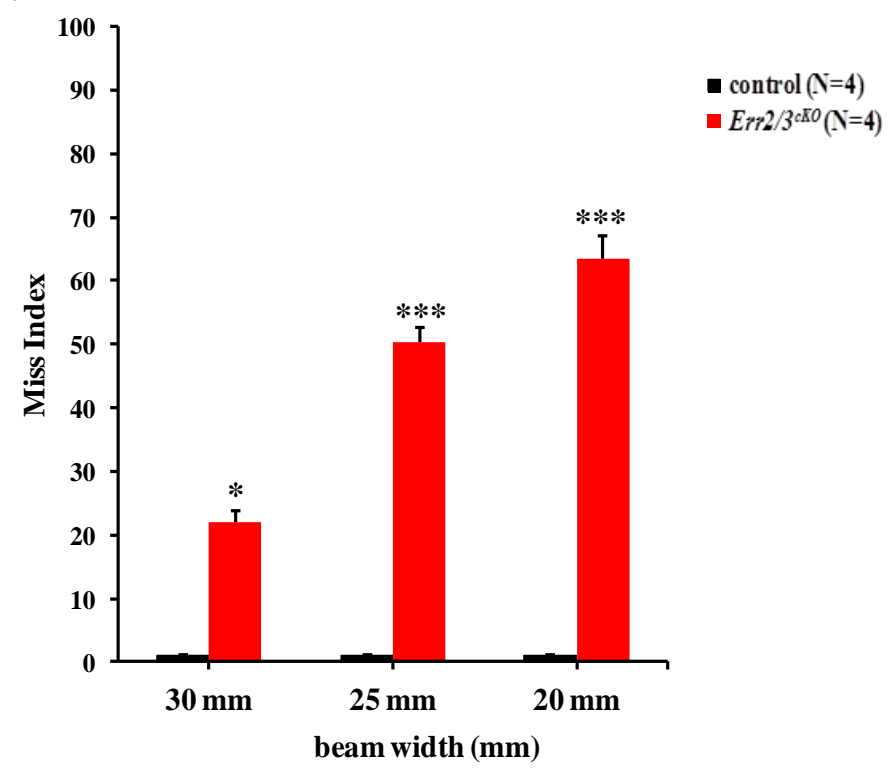

Figure 3.16 Err $2 / 3^{c K O}$ show precision movement deficits when challenged with horizontal beam task

(A) Video images of mice walking across a horizontal beam. The task was scored using specific scoring criteria: 1) "hit" of hind paw is scored when the animal had a flat and stable position on the beam (white arrow) or 2) when the hind paw straddled the side of the flat beam with a grip (white 
arrow). 3) A "miss" is scored when the hind paw slipped/hanged on the beam without a grip onto the side of the beam (red arrow) or when the mouse failed to locate the beam. (B) Bar graph showing the quantification of hindlimb stepping errors for control and $E r r 2 / 3^{c K O}$ mice when challenged with the horizontal beam task. Err $2 / 3^{c K O}$ committed more mean misses by a factor of $>22,>49$ or $>62$ at the beam widths of 30,25 or $20 \mathrm{~mm}$, respectively. Thus, Err $2 / 3^{c K O}$ mice show impaired precision movements and the data suggests that stepping errors increased with the increase in the difficulty of the task.

\subsection{Err2 and Err3 function in driving chick MN biophysical properties}

The capacity of forced Err2 and Err3 expression to drive chick MN biophysical properties into a specific MN subtype was studied using whole cell patch-clamp recordings carried out on acute chick spinal cord slices. In ovo electroporation was used to stably express several constructs into chick MNs through the Tol2 transposon-mediated system (Fig. 2.6 in Materials \& Methods section 2.13.1) (Kawakami and Shima, 1999; Kawakami, 2007; Sato et al., 2007). Several different constructs were electroporated into the chick neural tube for electrophysiological recordings (Fig. 2.9 in Materials \& Methods section 2.14.2). The constructs carrying VP16 and EnR along with Err2 were used to test whether the increase of transcription factor activity or repression can influence biophysical properties, respectively (Materials \& Methods section 2.13.1). MNs were identified using GFP fluorescent signal in the ventral horn of the chick spinal cord and recorded.

The data for chick whole cell patch-clamp studies are summarized in Table 8.2 of Appendix 2. The data showed that the forced expression of Err2VP16 in MNs resulted in a much higher mean firing frequency with respect to current injection when compared to the MNs that expressed control eGFP (Fig. 3.17). Forced Err2 and Err3 expression in MNs showed increased mean firing frequency, however, the shift was not as robust as the forced expression of Err2VP16. Furthermore, forced expression of Err2EnR in MNs showed no changes in the firing frequency when compared to MNs that expressed control eGFP. 


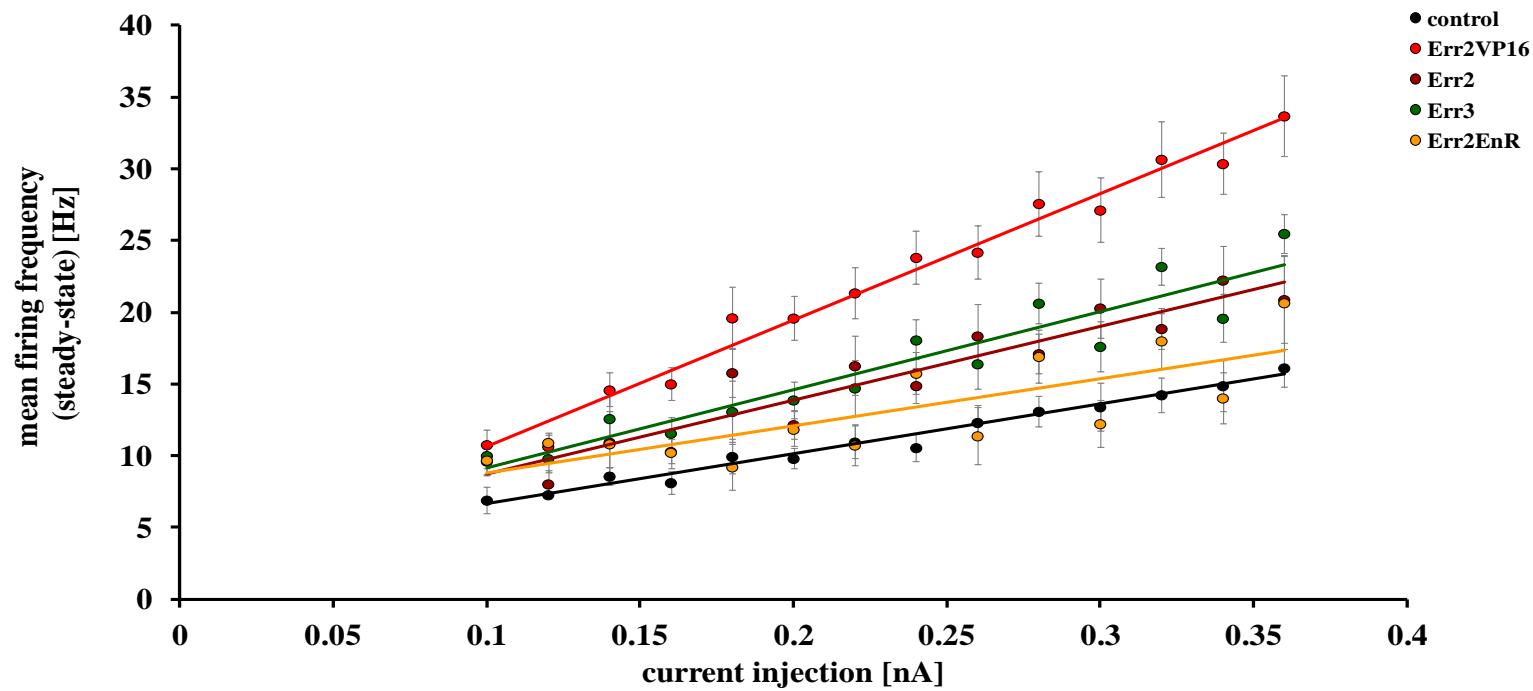

Figure 3.17 Forced expression of DNA constructs in MNs shifts their mean firing frequency

Several DNA constructs were electroporated: 1) control eGFP (CMV-eGFP), 2) Err2 (CMV-Err2$e G F P), 3) \operatorname{Err} 3(C M V$-Err3-eGFP), 4) Err2VP16 (CMV-VP16-Err2-eGFP), 5) Err2EnR (CMV-EnRErr2-eGFP). Scatter-plot showing current injection (pA) (x-axis) versus mean firing frequency $(\mathrm{Hz})$ (steady-state) (y-axis) in MNs that expressed either control eGFP, Err2VP16, Err2, Err3, or Err2EnR in MNs. The highest mean firing frequency shift with respect to current injection was observed in MNs that expressed Err2VP16. Thus, the highest shift in gain (Hz/nA) (steady-state) was observed with the forced expression of Err2VP16.

The data also showed that forced expression of VP16Err2 in MNs had the most robust shift in biophysical properties like mean rheobase (Fig. 3.18A), mean firing frequency (Fig. 3.18B) and mean gain (Fig. 3.18C). Thus, Err2VP16 promotes MN excitability towards a putative $\gamma$-MN phenotype of low rheobase, high firing frequency and high gain. 

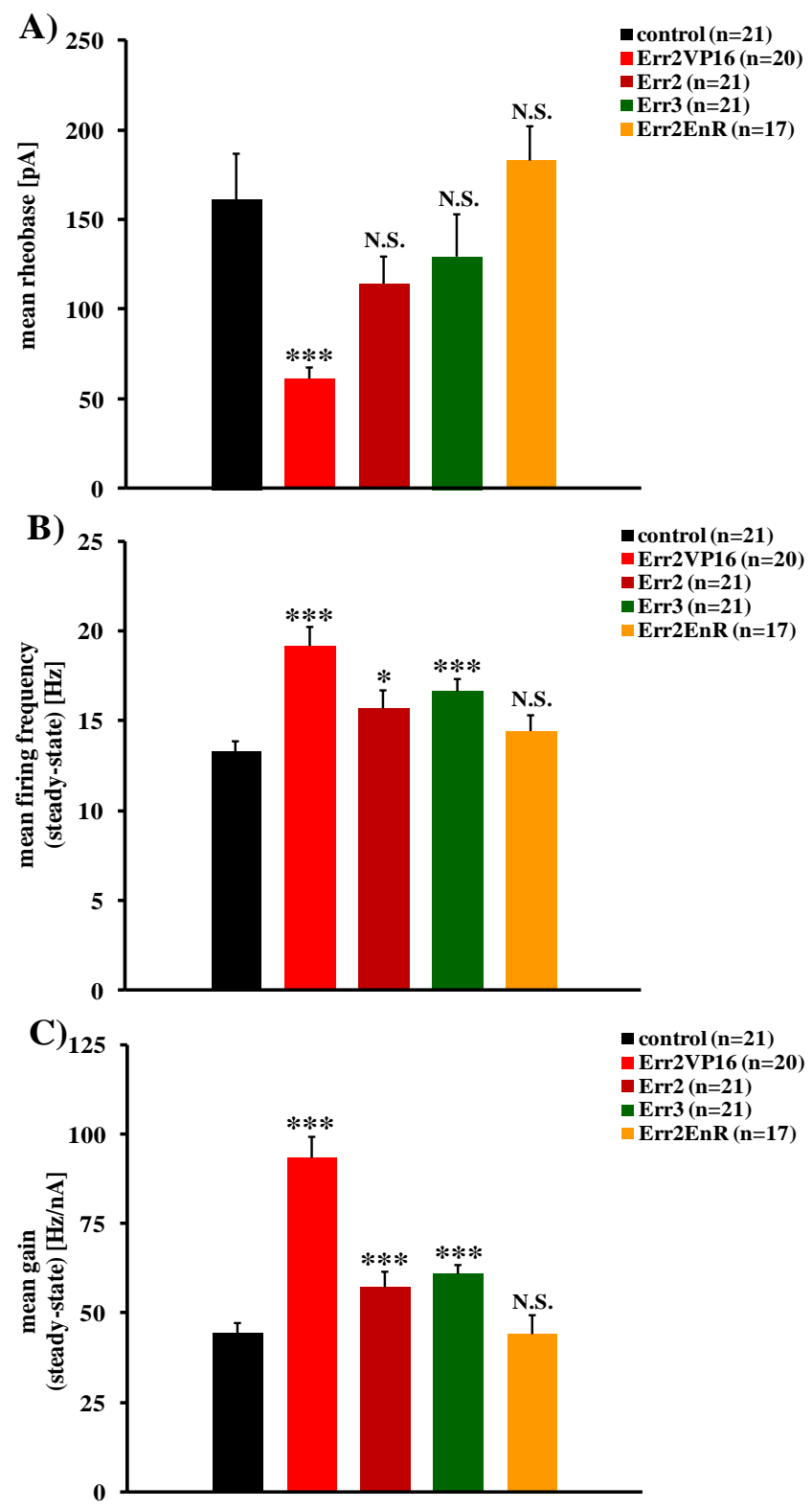

Figure 3.18 Forced expression of Err2VP16 in chick MNs drives their biophysical properties towards lower rheobase, higher firing frequency and gain

Several constructs were electroporated and the control eGFP (CMV-eGFP) construct was used to compare the effects of different DNA constructs (CMV-Err2-eGFP, CMV-Err3-eGFP, CMV-VP16Err2-eGFP, CMV-EnR-Err2-eGFP) on MN properties in chick. (A) The mean rheobase in MNs that expressed Err2VP16 was significantly lower than the mean rheobase for MNs expressed control eGFP. While the expression of Err2, Err3, EnR in MNs did not have any effects on the rheobase when compared to the expression of control eGFP (B) Furthermore, the mean firing frequency (steady-state) for MNs that expressed Err2VP16 was significantly higher than the mean firing frequency for MNs that expressed control eGFP. While the expression of Err2 and Err3 in MNs showed higher mean firing frequency when compared to MNs that expressed control eGFP, although the expression of EnR showed no significant effects compared to MNs that expressed control eGFP. (C) Similar to the mean firing frequency patterns, the mean gain (steady-state) for MNs that expressed Err2VP16 was significantly higher than the mean gain for MNs that expressed control eGFP. Overall, these data suggest that Err2VP16 influences the biophysical properties including rheobase, firing frequency and gain. 
Overall, the $F-I$ curve protocol of square current pulses to MNs showed significant differences in firing properties between the MNs that expressed Err2VP16 compared to the MNs that expressed control eGFP (Fig. 3.19A-F).

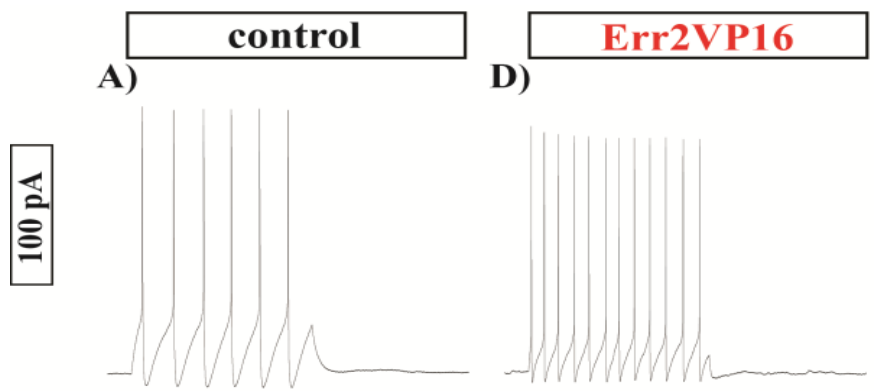

B)

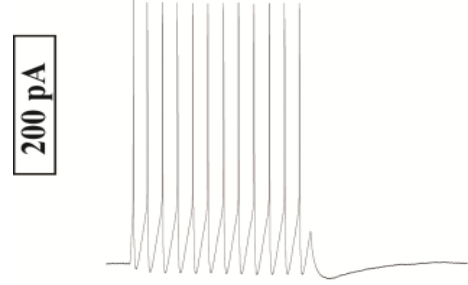

C)

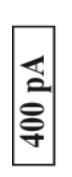

E)

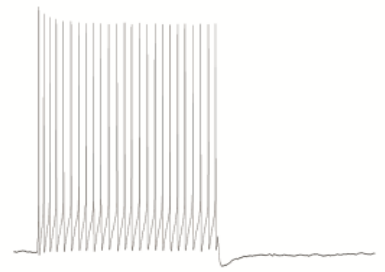

F)

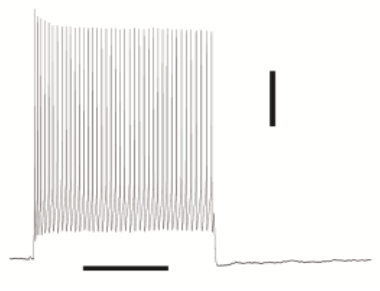

Figure 3.19 Forced expression of Err2VP16 in chick MN leads to a pattern of significantly higher firing frequency and higher gain

(A-F) Representative voltage response traces in MNs due to increments of $20 \mathrm{pA}, 1000 \mathrm{~ms}$ square current pulses during F-I curve square current pulse protocol. (A, D) A 100 pA, $1000 \mathrm{~ms}$ square current pulse leads to a higher firing frequency in MN that expressed Err2VP16 when compared to MN that expressed control eGFP. (B, E) This pattern continues when a $200 \mathrm{pA}, 1000 \mathrm{~ms}$ square current pulse is applied. (C, F) The firing frequency of the MN that expressed Err2VP16 increased still more with a $400 \mathrm{pA}, 1000 \mathrm{~ms}$ square current pulse, thus, showing higher gain (rate of change in firing frequency).

Like the mouse studies, scatter-plots comparing rheobase versus mean firing frequency or gain show a clear segregation of MNs that expressed Err2VP16 (lower rheobase, higher mean firing frequency, higher gain) from the MNs that expressed control eGFP (high rheobase, low mean firing frequency and low gain) (Fig. 3.20A, B). 

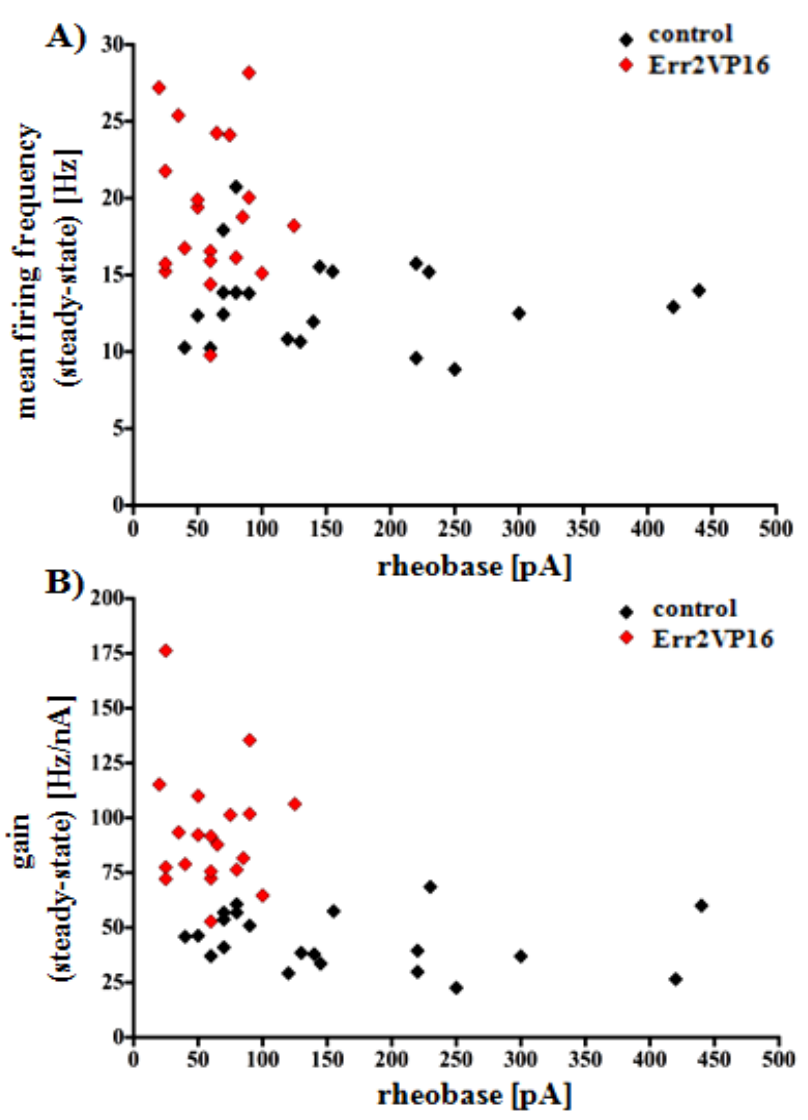

Figure 3.20 Forced expression of Err2VP16 in chick MNs drives their biophysical properties towards lower rheobase, higher firing frequency and gain

(A) Scatter-plot showing rheobase (pA) (x-axis) versus mean firing frequency (Hz) (steady-state) (y-axis) in MNs that express either control eGFP or Err2VP16 MNs. A distinct cluster of shifted MNs with low rheobase (pA) and high mean firing frequency $(\mathrm{Hz})$ is observed in MNs that expressed Err2VP16 when compared to MNs that expressed control eGFP. (B) Scatter-plot showing rheobase (pA) (x-axis) versus gain (Hz/nA) (steady-state) (y-axis) in the same MNs. Similarly, high gain $(\mathrm{Hz} / \mathrm{nA})$ is observed in the MNs that were expressed Err2VP16 when compared to MNs that expressed control eGFP. Scale bars represent $0.5 \mathrm{~s}$ duration of the square current pulse and $20 \mathrm{mV}$ of voltage-response upon the current pulse.

\subsection{The mechanistic role of Err2 in the influence of $\gamma$-MN biophysical properties}

The next logical step was to identify Err2-target genes through which it promotes $\gamma$ MN biophysical properties. Therefore, RNA sequencing studies were designed and bioinformatic analyses using the JASPAR CORE containing published transcription factor binding site profile for eukaryotic organisms were carried out (http://jaspar.genereg.net/; Matheliar et al., 2016).

\subsubsection{Err2 up-regulates Kcna10 gene expression}

In order to understand Err2 actions in a broader context, RNA sequencing experiments were performed by isolating electroporated motor columns from the chick neural tube at E12.5 (HH St. 38-39) (Fig. 2.8 in Materials \& Methods section 2.13.4). Err2VP16 
differential gene expression was compared with Delta-like homolog 1 (Dlk1) differential gene expression in the chick neural tube because Dlk1 was recently identified as necessary and sufficient in promoting a fast $\alpha-\mathrm{MN}$ biophysical signature (Müller et al., 2014). At E12.5 (HH St. 38-39), chick spinal motor columns showing eGFP expression were dissected to extract the RNA (Materials \& Methods section 2.13.4). Gene expression analysis indicated several genes that showed significant differential expression in the motor columns of chick embryos that expressed either control eGFP, Err2VP16 or Dlk1 (Fig. 3.21). Interestingly, chick Kcna10 was up-regulated by Err2VP16 when compared to the RNA profile of control eGFP and Dlk1. Thus, the data suggest that Err2 regulates the expression of KcnalO in chick spinal motor columns.

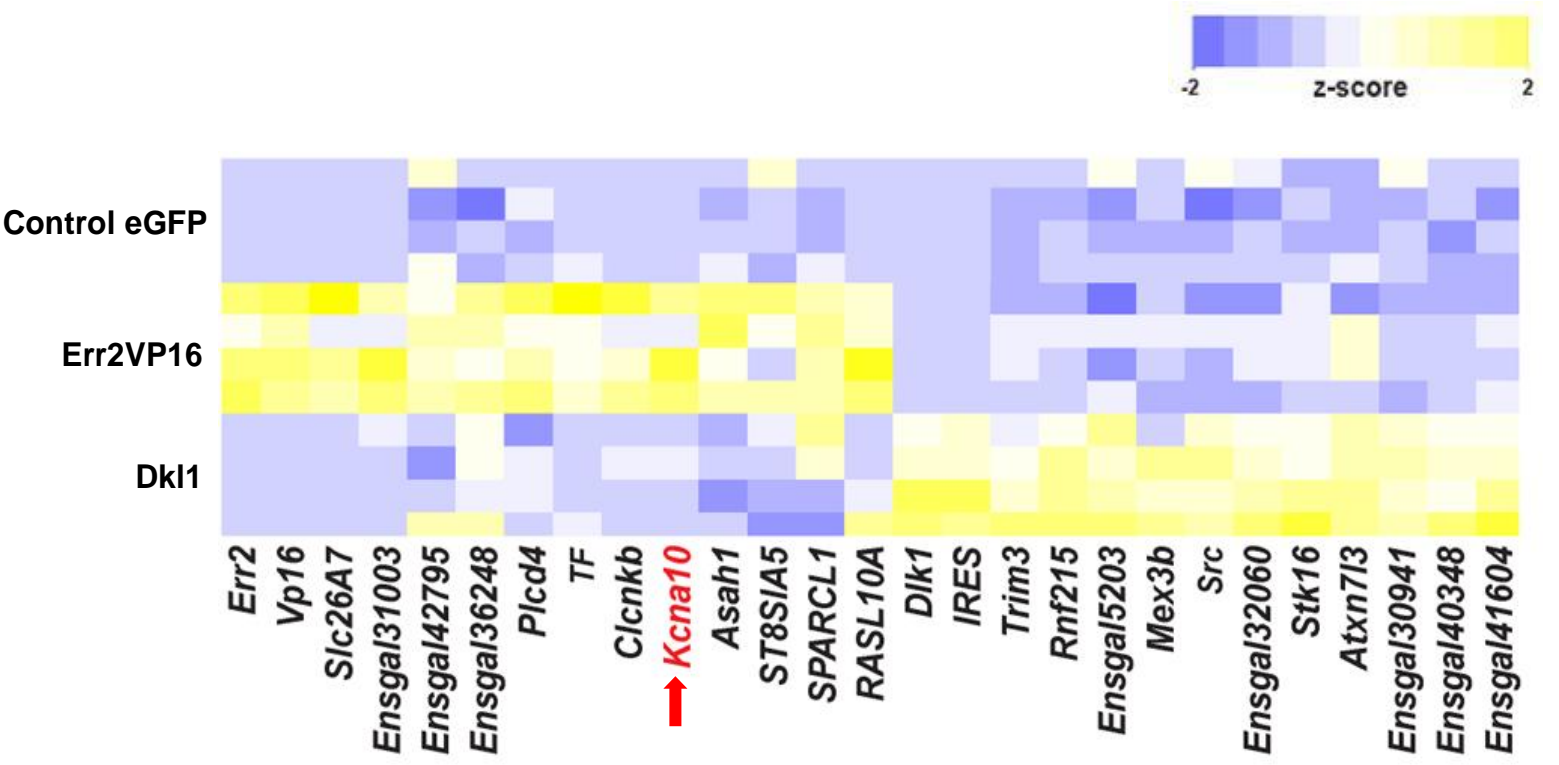

Figure 3.21 Forced expression of CMV-VP16-Err2-eGFP up-regulates Kcna10 RNA in chick spinal columns at E12.5 (HH St. 38-39)

Different DNA constructs were electroporated for the RNA sequencing experiment: 1) control eGFP (CMV-eGFP), or 2) Err2VP16 (CMV-VP16-Err2-eGFP) or 3) Dlk1 ( $p C A G G S-D l k-1-I R E S-e G F P)$. Differential transcript reads depicted by a Heat-map when control eGFP, or Err2VP16 or Dlk1 is electroporated into chick motor columns. Up-regulated gene expression is shown in yellow, while down-regulated gene expression is shown in blue (z-score): (-2.0 to 2.0). Chick Kcnalo (solid red arrow), was differentially expressed between control eGFP and Err2VP16. Err2VP16 up-regulates chick Kcna10 (red arrow). The heat map includes several comparisons: 1) genes which are upregulated $(p<0.05)$ in Err2VP16 (versus control eGFP) and up-regulated in Err2VP16 (versus Dlk1), 2) genes which are up-regulated $(p<0.05)$ in Dlk1 (versus control eGFP) and up-regulated in Dlk1 (versus Err2VP16). (The embryos were electroporated and dissected by Mudassar N. Khan, the RNA sequencing was performed by Ashish Rajput and the analysis was performed by Dr. Vikas Bansal) 


\subsubsection{Err2 directly regulates Kcna10 gene activity}

Bioinformatic and quantitative immunodetection studies were used to analyze whether Err2 directly influences Kcna10 gene activity. Screening studies in Japanese patients with heart arrhythmias (long QT syndrome, LQTS) showed genetic variations or Singlenucleotide polymorphisms (SNPs) in Kcna10, thus, it was suggested that KcnalO may be important for action potential properties in cardiomyocytes (Iwasa et al., 2001). Furthermore, Kcna10 is expressed in hair cells in mouse inner ear and Kcnal0-deficient mice show vestibular and hearing impairments (Lee et al., 2013). Evolutionarily conserved regions (ECRs) located upstream of the Kcnal0 gene were identified using the ECR Browser (Materials \& Methods section 2.13.3) (Ovcharenko et al., 2004). The mouse Kcna10 ECR contained three putative Err2/3 binding sites or motifs (Fig. 3.22). The ECR containing the Err2/3 binding sites was cloned into a DNA construct containing tdTomato sequence as a reporter for Kcnal0 gene activity (Materials \& Methods section 2.13.3). I analyzed whether Err2 may be able to bind its putative binding sites in the Kcnalo ECR and drive the expression of $t d$ Tomato reporter activity when this DNA construct is co-electroporated with Err2VP16 in chick motor columns (Fig. 2.7 in Materials \& Methods section 2.13.3). Moreover, a construct containing mutated Err2/3 motifs was also co-electroporated with Err2VP16 to test for $t d$ Tomato reporter expression (Fig. 3.22) (Materials \& Methods section 2.13.3).

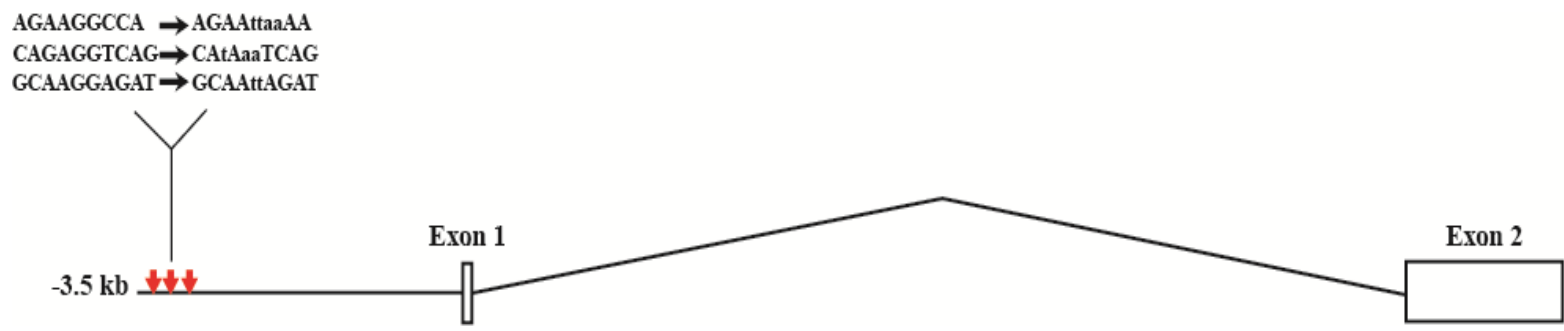

Figure 3.22 Schematic of Kcna10 genomic locus showing the putative Err2/3 bindings sites in the Kcna10 ECR

Site-directed mutagenesis by PCR was used to make substitution mutations in the three putative Err2/3 binding sites in the mouse Kcnal0 ECR (red arrows): 1) AGAAGGCCA to AGAAttaaAA, 2) CAGAGGTCAG to CAtAaaTCAG, 3) GCAAGGAGAT to GCAAttAGAT.

Quantitative immunodetection studies were performed on chick E6.5 (HH St. 28-29) spinal cord cryosections treated with anti-GFP and anti-DsRed primary antibodies and appropriate secondary antibodies. These cryosections were then imaged using a confocal microscope and quantified (Fig. 3.23A-I) (Materials \& Methods section 2.12.6). 


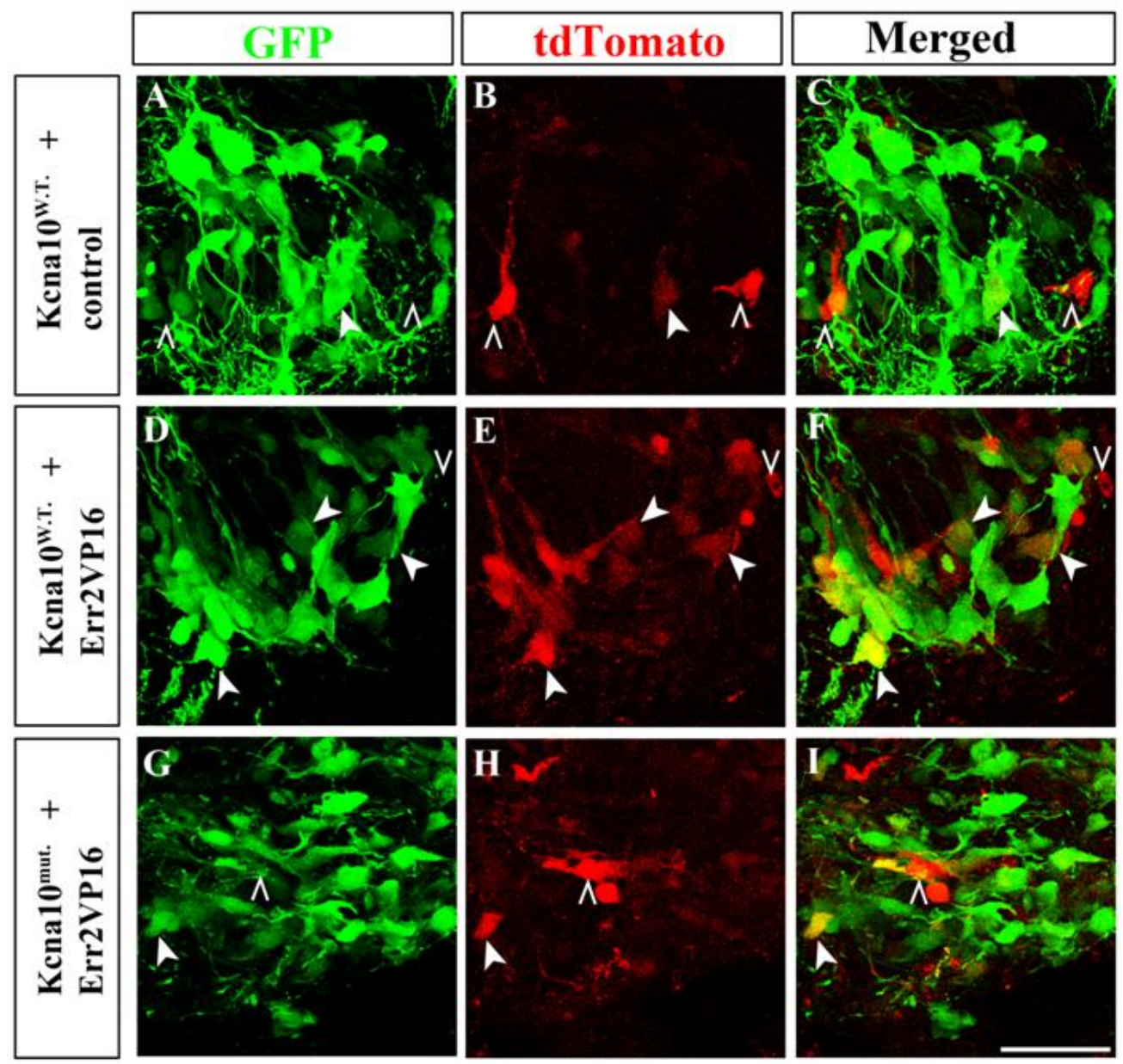

Figure 3.23 Err2 regulates chick Kcna10 gene activity in MNs

(A-I) Representative confocal microscope images showing ventral horn of the chick neural tube at E6.5 (HH St. 28-29) co-electroporated with different DNA constructs. Cryosections of the embryos were incubated with anti-DsRed and anti-GFP antibodies. For all panels, tdTomato reporter expression is seen in red and GFP expression is seen in green. (A-C) Kcna10 ${ }^{\text {W.T. }}+$ control eGFP coelectroporation resulted in some cells with tdTomato reporter expression in the $\mathrm{GFP}^{+}$cells (indicated by solid arrow), while majority of the cells that had high expression levels of endogenous tdTomato were also GFP cells (indicated by hollow arrows). (D-F) When Kcna10 $^{\text {W.T. }}+$ Err2VP16 were coelectroporated, there was a robust increase the expression levels of tdTomato in the $\mathrm{GFP}^{+}$cells (indicated by solid arrows). While there were still some endogenous tdTomato reporter expression GFP $^{-}$cells (indicated by hollow arrows) (G-I) When Kcna10 ${ }^{\text {mut. }}+$ Err2VP16 were co-electroporated, there was an increase in tdTomato reporter expression in $\mathrm{GFP}^{+}$(indicated by solid arrow), however the expression levels were not as elevated as compared to the co-electroporation of Kcna10 ${ }^{\text {W.T. }}+$ Err2VP16. The scale bar represents $50 \mu \mathrm{m}$.

The data quantification showed that electroporation of Err2VP16 significantly elevated the expression levels of Kcnal0 gene activity reporter, tdTomato (Fig. 3.24). While the electroporation of Err2VP16 with the three binding sites mutated in Kcna10 ECR showed significantly decreased expression levels of tdTomato. Thus, the data suggest that Err2 directly binds Kcna10 ECR containing the Err2/3 binding sites and regulates KcnalO gene activity. 


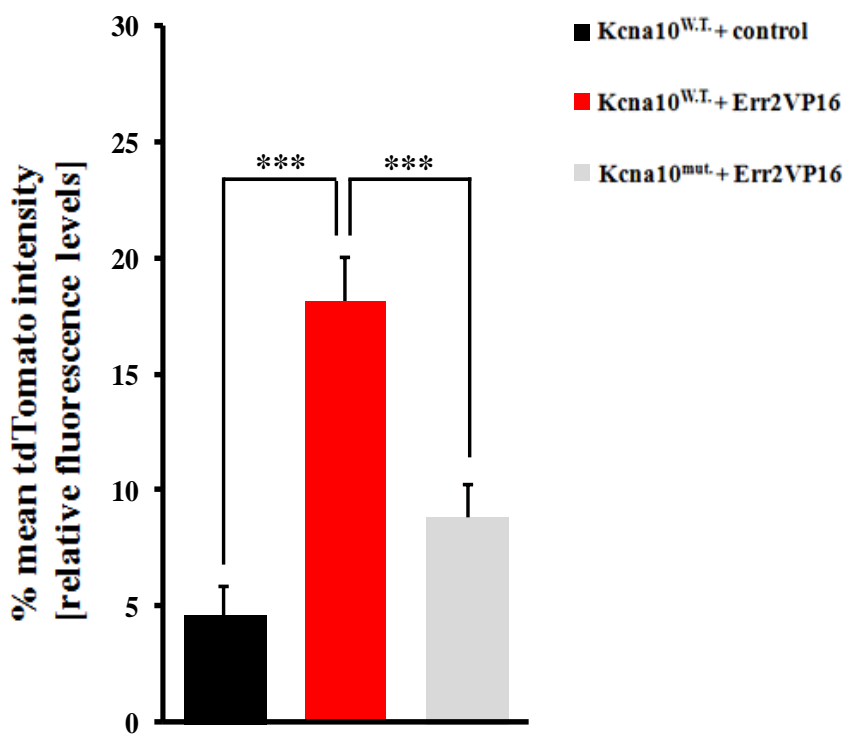

Figure 3.24 Forced expression of Err2VP16 increases Kcna10 gene activity

Significantly robust normalized \% mean tdTomato intensity in relative fluorescence levels was observed when Kcna10 ${ }^{\text {W.T. }}$ DNA construct was co-electroporated with Err2VP16 DNA construct than when Kcna10 ${ }^{\text {W.T. }}$ was co-electroporated with control eGFP DNA construct. While significantly less elevated normalized \% mean tdTomato intensity in relative fluorescence levels was observed when Kcna10 ${ }^{\text {mut. }}$ DNA construct was co-electroporated with Err2VP16 DNA construct than when Kcna10 ${ }^{\text {W.T. }}$ and Err2VP16 DNA constructs were co-electroporated. The data showed that the cells that co-expressed Kcna10 ${ }^{\text {W.T. }}$ and Err2VP16 (mean \pm S.E.M., $\mathrm{n}=$ neurons, $\mathrm{N}=$ embryos, $p=p$-value; $18.14 \pm 1.85, \mathrm{n}=103$, $\mathrm{N}=3, p=6.9 \mathrm{e}^{-9}$ ) resulted in significantly higher mean tdTomato expression levels compared to the cells that co-expressed Kcna10 ${ }^{\text {W.T. }}$ and control eGFP $(4.57 \pm 1.23, \mathrm{n}=101, \mathrm{~N}=3)$. Moreover, the co-expression of Kcna10 ${ }^{\text {mut. }}$ and Err2VP16 $\left(8.85 \pm 1.38, \mathrm{n}=102, \mathrm{~N}=4, p=8.63 \mathrm{e}^{-5}\right)$ resulted in significantly less elevated mean tdTomato expression levels in the cells when compared to the cells that co-expressed Kcna10 ${ }^{\text {W.T. }}$ and Err2VP16. Thus, the data suggests that Err2 regulates Kcna10 gene activity by binding three motifs in the Kcnalo ECR.

\subsubsection{Kena10 biophysical properties in chick MNs}

Potassium channels have been implicated in the regulation of membrane potential and action potentials of excitable cells, I therefore tested the role of Kcna10 in influencing MN biophysical properties in the chick. Thus, a DNA construct carrying chick Kcna10 ( $C M V$ Kcna10-eGFP) was electroporated into chick embryo MNs and electrophysiological recordings were performed (Fig. 2.10 in Materials \& Methods section 2.14.2). Biophysical properties from the whole cell patch-clamp studies were analyzed (Summary provided in Table 8.2 of Appendix 2). These data suggest that the forced expression of Kcna10 partially drives $\mathrm{MN}$ biophysical properties towards lower rheobase (Fig. 3.25A), higher firing frequency (Fig. 3.25B), higher gain (Fig. 3.25C), which is similar to the shift in biophysical properties observed when Err2VP16 is expressed in MNs. 


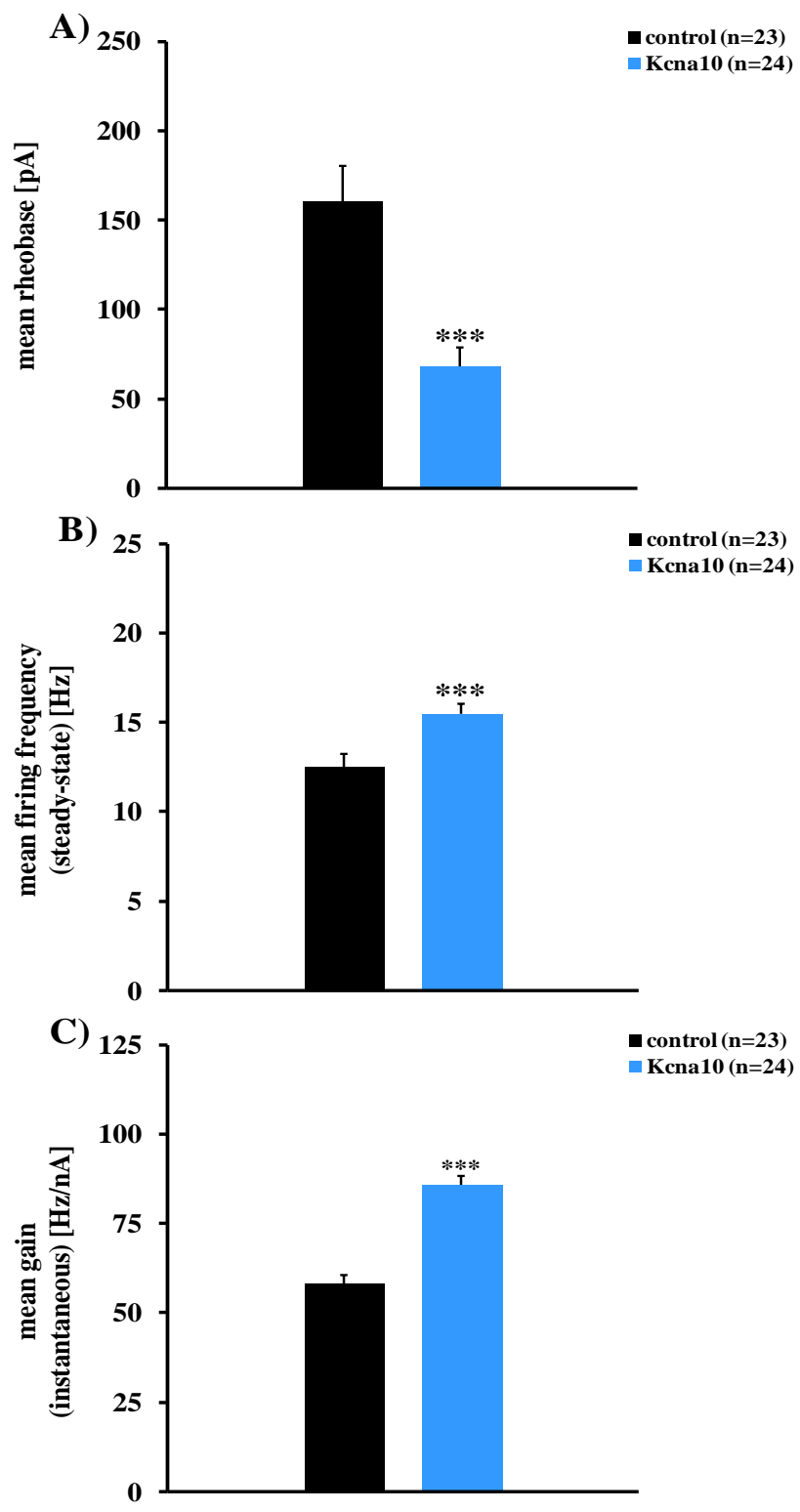

Figure 3.25 Forced Kena10 expression in chick MNs promotes $\gamma$-MN biophysical signature

(A) The mean rheobase for Kcna10 (CMV-Kcna10-eGFP) expressing MNs was significantly lower than the mean rheobase for MNs that expressed control eGFP (CMV-eGFP). B) The mean firing frequency (steady-state) for Kcna10 expressing MNs was significantly higher than the mean firing frequency for MNs expressing control eGFP. C) The gain (instantaneous) for Kcna10 expressing MNs was significantly higher than for MNs expressing control eGFP.

The comparison of mean rheobase versus mean firing frequency or gain (instantaneous) in scatter-plots showed a clear segregation of MNs that expressed Kcna10 (which showed lower rheobase, higher mean firing frequency, higher gain (instantaneous)) when compared to MNs that expressed control eGFP (Fig. 3.26A, B). 


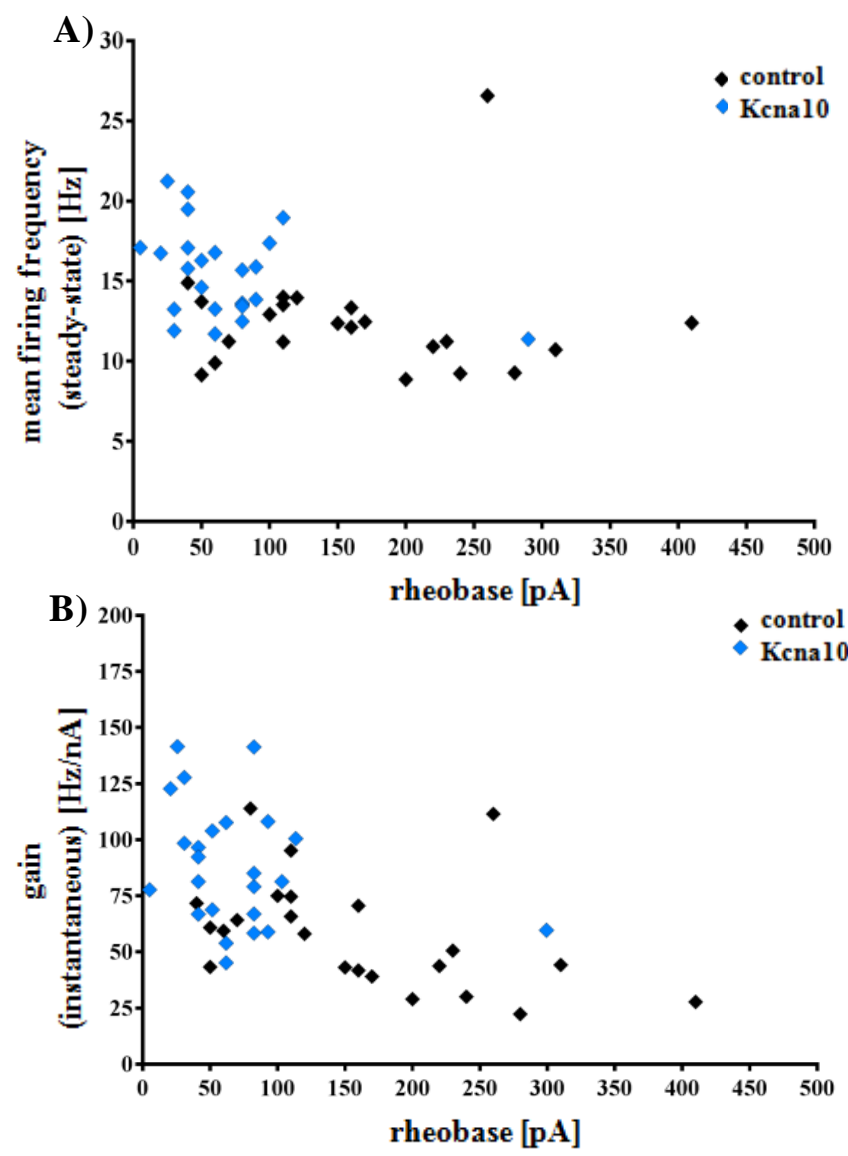

Figure 3.26 Forced expression of Kena10 in chick MNs to a shift in biophysical properties towards lower rheobase, higher firing frequency, and higher gain

(A) Scatter-plot showing rheobase (pA) (x-axis) versus mean firing frequency (Hz) (y-axis) in control eGFP $(C M V-e G F P)$ versus Kcna10 (CMV-Kcna10-eGFP) MNs. MNs expressing Kcna10 shift towards lower rheobase $(\mathrm{pA})$ and higher mean firing frequency $(\mathrm{Hz})$ in comparison to $\mathrm{MNs}$ that express control eGFP. (B) Comparing rheobase (pA) (x-axis) versus gain (instantaneous) (Hz/nA) (yaxis), Kcna10 drives chick MNs to have a lower rheobase (pA) and higher gain (instantaneous) $(\mathrm{Hz} / \mathrm{nA}))$. 


\section{DISCUSSION}

Spinal motor neurons (MNs) generate and control movements. Alpha MNs ( $\alpha$-MNs) signal to generate force during movement, while gamma MNs ( $\gamma-\mathrm{MNs})$ regulate the sensitivity of the muscle spindle (MS) apparatus that is involved in muscle reflexes and muscle proprioception during movement. The circuit consisting of $\gamma$-MNs, intrafusal fibers, and Ia sensory afferents within the MS apparatus gauges the overall change and the rate of change in muscle length. Thus, the MS provides proprioceptive information to the nervous system about limb position and muscle velocity. The nervous system's capacity to control the MS through $\gamma$-MN innervation has evolved in higher vertebrates, which enables them to perform intricate and precise motor behaviors. Ultimately, $\gamma$-MN biophysical properties and therefore their function in controlling the MS are shaped by the action of specific genes. However, at the beginning of my thesis there was no mechanistic understanding of the acquisition of $\alpha$ - versus $\gamma-\mathrm{MN}$ functional diversity. Therefore, this study was designed to identify molecular mechanisms that endow MNs with specific biophysical properties, which in turn allow them to function in spinal networks to regulate motor behavior in mice.

\subsection{Molecular characteristics of $\gamma$-MNs}

The $\alpha$ - and $\gamma$-MNs likely express distinct sets of genes, which shape their biophysical properties and their function. Thus, to understand how $\alpha$ - and $\gamma$-MNs diversify functionally, my initial studies focused on assessing the developmental expression pattern of unique molecular correlates in the mouse spinal cord. Previously, studies have demonstrated that $\gamma$ MNs have high estrogen-related receptor 3 (Err3) expression levels and low expression levels of NeuN, while $\alpha$-MNs have low or negligible Err3 expression levels and high NeuN expression levels (Friese et al., 2009). In another study, estrogen-related receptor 2 (Err2), a closely related paralogue of Err3, was suggested to be expressed by slow $\alpha$-MNs (Enjin et al., 2010). Because both Err2 and Err3 are molecularly very similar, I reexamined the expression pattern of Err2 in relation to that of Err3 in W.T. (CD1 strain) mice through quantitative immunodetection. My studies showed that at postnatal day 21 (P21), Err2 and Err3 were coexpressed at high levels in relatively small soma size MNs that also had low expression levels of NeuN and incorporated high levels of Fluoro-Gold (FG) retrograde tracer.

Furthermore, quantitative immunodetection studies carried out in FG injected W.T. mice at P4, P14 and P21 suggested that Err2 and Err3 are co-expressed at high levels in relatively small soma size MNs that incorporated high FG levels and expressed low NeuN 
(Rbfox3) levels. More specifically, high Err2 and Err3 expression levels become restricted to $\gamma$-MN populations beginning at $\mathrm{P} 14$ and become completely restricted by $\mathrm{P} 21$. Thus, my data supports the finding by Friese et al., 2009 that $\gamma$-MN populations express distinct molecular correlates that refine in the first two weeks of postnatal development.

Previously, using RNA in situ hybridization and immunodetection studies, Enjin et al., (2010), suggested that Err2 may be a molecular correlate for slow $\alpha$-MNs. Enjin et al., (2010), observed that Err2 was expressed in small soma size cholinergic $\left(\mathrm{ChAT}^{+}\right)$and noncholinergic cells (Enjin et al., 2010). The authors proposed that Err2 is not likely a $\gamma$-MN molecular correlate since RNA in situ hybridization experiments in control versus $\mathrm{Egr3}^{-/}$ mutant mice at P11 revealed that Err2 transcript expression was not overtly different. The authors reasoned that altered Err2 expression levels should have been observed in $\mathrm{Egr}^{-/}$ mutant mice, since the mice had degenerated MSs that resulted in $\gamma$-MN degeneration due to the lack of trophic factors from the MSs (Enjin et al., 2010). My data from the quantitative immunodetection studies however showed that Err2 is expressed by three distinct populations of cells in the ventral spinal cord: 1) the putative $\gamma$-MNs expressed high Err2 and Err3 levels, low NeuN levels and incorporated high levels of FG, 2) the putative $\alpha$-MNs expressed low Err2 and Err3 levels, moderate-to-high NeuN levels and incorporated low levels of FG and 3) non-cholinergic cells expressed high Err2 and Err3 levels, high NeuN levels and incorporated no FG. It is possible that the non-cholinergic populations of cells comprising of interneurons and/or glial cells may be the source of Err2 ${ }^{+}$cells identified in the RNA in situ hybridization studies in $\mathrm{Egr}^{-/-}$mice performed by Enjin et al., 2010. Therefore, I infer that Err2 was misassigned as an $\alpha-\mathrm{MN}$ molecular correlate due to the complex expression pattern in these cell populations.

My studies therefore show that Err2 and Err3 are co-expressed at high levels in putative $\gamma$-MNs. These observations also suggest that the expression pattern of molecular markers or transcription factors, like Err2 and Err3 in spinal MNs may be more complex than previously suggested, which may shape the functional properties of different neural subtypes in the spinal cord. Studies have shown that combinations of transcription factors regulate the diversity of cell fates during development ( $\mathrm{Lu}$ et al., 2015). Thus, it is possible that the functional diversity of neurons other than MNs is also regulated by a combinatorial pattern of gene expression. 


\subsection{Distinct biophysical properties of $\alpha$ - and $\gamma$-MNs in mice}

Mature $\gamma$-MN biophysical properties were previously recorded in the cat 40 years ago (Kemm and Westbury, 1978). The fortuitous finding that small soma size putative $\gamma$-MNs incorporate high levels of FG retrograde tracer in their somas enabled me to identify and record mature mouse $\gamma$-MNs from the ventral horn of the spinal cord. Moreover, my studies also showed that the putative $\gamma$-MNs that incorporated high levels of FG also lacked the Ia sensory afferent connectivity, which is consistent with previous findings that showed that $\gamma$ MNs lack Ia sensory afferent innervation (Eccles et al., 1960; Friese et al., 2009). Initially, I performed whole cell patch-clamp recordings in $\mathrm{FG}^{\text {high }}$ (putative $\gamma$-MNs) and $\mathrm{FG}^{\text {low }}$ (putative $\alpha-\mathrm{MNs}$ ) from control mice to determine the baseline differences in their biophysical properties. In agreement with various studies in cat (Kemm and Westbury, 1978), my recordings in mice confirm that $\alpha$ - and $\gamma$-MNs have distinct biophysical properties, which are likely important for generating force and movements by $\alpha$-MNs and for proprioception, stretch reflex and the patterns of locomotion by $\gamma$-MNs.

\subsection{The role of Err2 and Err3 in driving $\gamma$-MN biophysical properties}

The data from my whole cell patch-clamp recordings showed that $\alpha$ - and $\gamma$-MNs had distinct biophysical properties in control mice. Therefore, I used the levels of FG and relative soma size in the ventral horn of the spinal cord to guide my recordings of the putative $\alpha$ - and $\gamma$-MNs from $\operatorname{Err} 2 / 3^{c K O}$ mice. In order to characterize the $E r r 2 / 3^{c K O} \mathrm{MNs}$, I decided to focus on firing frequency, gain and rheobase to distinguish putative $\gamma$-MNs from putative $\alpha$-MNs since these properties are thought to segregate them. My data showed that the putative $\gamma$-MNs from $E r r 2 / 3^{c K O}$ at postnatal days (P20-P22) have higher rheobase and lower firing frequency when compared to the putative $\gamma$-MNs in control MNs at the same ages. This data suggested that the lack of Err2 and Err3 expression in the putative $\gamma$-MNs of Err2/3 ${ }^{c K O}$ mice results in a shift in their biophysical properties towards $\alpha$-MN-like. Moreover, the data showed no significant differences in the biophysical properties of the putative $\alpha$-MNs from $E r r 2 / 3^{c K O}$ versus control mice. Overall, I therefore concluded that Err2 and Err3 drive $\gamma-\mathrm{MN}$ biophysical signature.

Studies in mice have shown that MSs mature morphologically during the third week of postnatal development (Sonner et al., 2017). This is also consistent with the development of the neuromuscular system and the ability of mice to bear weight at about two-weeks of age postnatally (Jiang et al., 1999). By this developmental stage, $\gamma$-MNs are functionally mature since they are tuned to control the sensitivity of the mature MSs. Since mice have essentially 
mature MSs at P20-P22 and the expression pattern of Err2 and Err3 is more restricted to $\gamma$ MNs, the recordings I performed were from mature $\gamma$-MNs. When mice begin to walk with bearing weight (P8-P11), they have significantly different action potential properties and properties like rheobase (higher), input resistance (lower), AHP half-decay times (lower), however, no significant changes in gain are observed when compared to mice at P3-P5 (Durand et al., 2015). Thus, MN biophysical properties change significantly during this developmental period.

Previous recordings in P0-P6 mice found immature $\gamma$-MN biophysical properties to be between fast and slow $\alpha$-MNs (Enjin et al., 2012), while intracellular recordings in young decerebrate cat have demonstrated that $\gamma$-MNs have higher discharge rates and lower discharge thresholds than $\alpha$-MNs (Kemm and Westbury, 1978). My findings were consistent with the latter studies despite the differences in the model organism and the recording technique. Thus, differences between the data from my recordings of $\gamma$-MN biophysical properties and those of Enjin et al., 2012, are likely due to the differences in developmental stage of the mice. Hence, this suggests that the maturation of MN properties, including the consolidation of ion channel expression, allows optimized tuning of the MS, thus, facilitates in the maturation of locomotor behavior observed between P6-P20 in mice. In summary, my recordings show that $\gamma$-MN properties can be distinguished at early postnatal stages based on the pattern of gene expression, but acquire their typical biophysical properties later (between P10-P20), matching the maturation of posture and movement in mice.

\subsection{The role of Err2 and Err3 in determining mouse gait and precision movements}

The proprioceptive information provided by the MS apparatus is important for normal gait and precision movements in vertebrates. Recently, studies have shown that Egr3deficient mice, which do not develop MSs, show gait deficits (Akay et al., 2014). However, the specific contribution of intact $\gamma$-MNs to control proprioception and gait in vivo has not been directly investigated. Thus, I performed behavioral studies to observe gait, posture and precision movements in $E r r 2 / 3^{c K O}$ mice.

Previous studies in cat have shown that proprioceptive feedback information is important for stance-swing phase transition of the stepping cycle (Grillner and Rossignol, 1978; Duysens and Pearson, 1980; Hiebert et al., 1996; Lam and Pearson, 2001; Mcvea et al., 2005). Moreover, the lack of proprioceptive information due to the lack of MS development in Egr3-deficient mice leads to altered muscle activities in ankle flexor and thus, produces an abnormal gait pattern (Akay et al., 2014). My data from the treadmill task showed that 
control and Err $2 / 3^{c K O}$ mice could be distinguished by their gait patterns and distinct gait variables or parameters that contributed to the differences observed in the gait patterns. For instance, there was reduced stance-swing ratio in Err $2 / 3^{c K O}$ mice, which suggests a shift in gait phase and implies that $\gamma$-MNs are important for precise gait patterns in mice.

Furthermore, studies have shown that the mechanism of co-activation of $\alpha$-MNs and $\gamma-\mathrm{MN}(\alpha-\gamma$ co-activation) occurs during the control of movement and posture (Vallbo, 1971; Taylor et al., 2004; 2006; Prochazka and Ellaway, 2012; Li et al., 2015). Data from my studies showed that $E r r 2 / 3^{c K O}$ mice displayed decreased stance widths, which imply postural changes in the mice. My studies suggest that the lack of proprioceptive input from the MSs due to the loss of $\gamma$-MN biophysical properties in $E r r 2 / 3^{c K O}$ mice results in the alteration of posture, thus, $\gamma$-MNs are essential for the control of posture. Moreover, this suggests that $\gamma$ MNs regulate the mechanism of $\alpha-\gamma$ co-activation to control posture in mice.

MSs appear in higher numbers in vertebral and lumbrical muscles that are vital for generating fine movements and the soleus muscle which is important for postural control (Barker, 1974; Fitz-Ritson, 1982). My data showed that $E r r 2 / 3^{c K O}$ mice had higher number of stepping errors while navigating the horizontal ladder and horizontal beam when compared to control mice, therefore, suggesting that $\gamma$-MN function is integral to performing precision movements.

Studies have shown that in mice, as in most other animals, gait patterns change with locomotion speed (Bellardita and Kiehn, 2015). Moreover, locomotion is generated by the central pattern generator (CPG) networks independent of the sensory information, however, proprioceptive information is needed to adjust CPG network activity, in order to adapt their locomotion to changes encountered in the environment (Grillner, 2006). My data showed that Err $2 / 3^{c K O}$ mice were able to perform the same range of running speeds as control mice, albeit with gait alterations. Thus, Err $2 / 3^{c K O}$ mice are still able to generate movements or locomote despite lacking functional $\gamma$-MNs. To summarize, my studies suggest that normal $\gamma-\mathrm{MN}$ biophysical properties and ultimately, function is needed to regulate MS function in order to maintain proper gait and posture and execute precision movements.

\subsection{The role of Err2 and Err3 in driving a $\gamma$-MN biophysical signature}

I next hypothesized that Err2 and Err3 are involved in driving the MN biophysical properties towards a $\gamma$-MN-like signature. The whole cell patch-clamp recordings I performed in Err2 or Err3-electroporated chick MNs showed significant increases in the firing frequency and gain combined with lower rheobase. The data analyses suggested that MNs that 
expressed Err2VP16 had the highest shift in biophysical properties when compared to the MNs that expressed control eGFP. Specifically, the forced expression of Err2VP16, a fusion protein that results in high transcriptional activation, caused the most dramatic shift in biophysical properties towards lower rheobase, higher firing frequency, and higher gain compared to the forced expression of control eGFP. Moreover, the forced expression of Err2EnR, a fusion protein with transcription repressor activity did not recapitulate Err2, Err3 or Err2VP16 actions on MN biophysical properties. I therefore concluded that Err2 alters biophysical properties through transcriptional activation.

Previous studies have demonstrated that $\alpha-\mathrm{MN}$-subtype biophysical properties can be regulated by Delta-like 1 homolog (Dlk1), which is expressed by fast $\alpha$-MNs from mice and is important for generating the fast $\alpha-\mathrm{MN}$ biophysical properties, including higher firing thresholds and firing frequencies, lower firing durations and after-hyperpolarization (AHP) (Müller et al., 2014). Studies in other organisms have shown that Islet, a LIM-Homeodomain (LIM-HD) transcription factor is sufficient in regulating Drosophila MN subtype excitability (Wolfram et al., 2012). In line with this evidence, my studies suggest that the expression of specific molecules like Err2/3 and their downstream actions can shape the functional properties of MN subtypes. Moreover, my studies have implicated a functionally redundant role of Err2 and Err3 in promoting $\gamma$-MN biophysical properties, thus, it may be fruitful to investigate whether other molecularly related genes may be involved in promoting functional diversification of MNs or other neural subtypes in the CNS.

\subsection{Working towards a molecular mechanism that generates $\alpha$ - and $\gamma$-MN functional diversification}

At the start of my thesis work, there was no known molecular mechanism that promoted $\alpha-\mathrm{MN}$ and $\gamma-\mathrm{MN}$ functional diversification. Therefore, RNA sequencing studies were performed using chick motor columns and the data showed that KcnalO expression was up-regulated in chick motor columns when the chick neural tube was electroporated with VP16Err2 when compared to control eGFP or Dlk1. Furthermore, Err2 target genes were different from those regulated by Dlk1, which is consistent since it was previously found to promote a fast $\alpha-\mathrm{MN}$ biophysical signature (Müller et al., 2014). In parallel, bioinformatic studies showed that evolutionary conserved region (ECR) of Kcnal0 contained the three putative Err2/3 binding sites. The Kcnal0 ECR containing the putative Err2/3 binding sites was cloned into a DNA construct containing tdTomato reporter gene. The fluorescence expression levels of tdTomato were used to quantify the ability of Err2VP16 to elevate 
reporter gene activity, which was under the control of Kcnal0 ECR. The data quantification of the immunodetection experiments showed that the expression of Err2VP16 significantly elevated the tdTomato reporter gene activity when compared to the expression of control eGFP. Moreover, the expression of Err2VP16 was not able to recapitulate this elevated tdTomato reporter gene activity when it was co-electroporated with KcnalO ECR containing mutated Err2/3 binding sites. Taken together, the experiments suggest that Err2VP16 can bind to the predicted binding sites in the KcnalO ECR and thereby mediate the increase in activity of tdTomato reporter gene through regulating the KcnalO ECR. To summarize, these studies suggest that there are distinct pathways that lead to the functional diversification of MNs that are regulated by genes like Err2/3 and $D l k 1$.

Previous studies have suggested that voltage-gated $\mathrm{K}^{+}$channels function to control frequency and duration of action potentials through repolarization (Humphries and Dart et al., 2015). Studies have also shown that voltage-gated $\mathrm{K}^{+}$channels regulate neuronal excitability (Murakoshi and Trimmer, 1999). Kcna10, a voltage-gated $\mathrm{K}^{+}$channel was intriguing due to its possible function in cardiomyocyte action potential (Lang et al., 2000; Iwasa et al., 2001). More specifically, studies have shown that $\mathrm{K}^{+}$ion currents set the discharge threshold and firing frequency in MNs (Leroy et al., 2015; Müller et al., 2014). My recordings showed that forced expression of Kcna10 in chick MNs resulted in decreased rheobase and increased firing frequency. Overall, my studies showed that expression of Kcna10, like the expression of Err2VP16, shifted MN biophysical properties towards $\gamma$-MN biophysical signature. Furthermore, studies that aim to characterize Kcna10 protein expression in mouse MNs may potentially provide evidence of whether Err2 regulates KcnalO gene activity to promote a $\gamma$ MN biophysical signature. My studies also imply that the expression of a specific type of voltage-gated $\mathrm{K}^{+}$channel may be responsible for the differences seen in the properties of $\alpha$ versus $\gamma$-MNs. Indeed studies have shown that the control of voltage-gated $\mathrm{K}^{+}$channel expression by transcription factor protein, Islet determines MN subtype specific biophysical properties (Wolfram et al., 2012). Thus, it is likely that the diversity of voltage-gated $\mathrm{K}^{+}$ channel expression may tune the functional properties of different MN subtypes.

\subsection{Err2 and Err3 actions specifically promote $\gamma$-MN function but not identity}

Previous studies had demonstrated that compared to $\alpha$-MNs, $\gamma$-MNs lacked Ia sensory innervation, thus, they lacked vesicular glutamate transporter 1 (vGlut1), which is used to package glutamate into vesicles and is a marker for Ia sensory afferents (Eccles et al., 1960; Friese et al., 2009; Oliveira et al., 2003). In agreement with previous findings, my data 
showed that $\gamma$-MNs essentially lacked Ia sensory afferent innervation, since they essentially lacked vGlut $1^{+}$synaptic varicosities apposed to $\mathrm{MN}$ soma and proximal dendrites when compared to $\alpha$-MNs from control mice. Like the $\gamma$-MNs from control mice, $\gamma$-MNs from Err $2 / 3^{c K O}$ mice essentially lacked vGlut $1^{+}$synaptic varicosities. Furthermore, no significant changes were observed in the number of vGlut $1^{+}$synaptic varicosities in $\alpha$-MNs from control versus Err2/3 ${ }^{c K O}$ mice. Thus, suggesting that the lack of Err2 and Err3 in Err2/3 ${ }^{c K O}$ mice does not alter Ia sensory afferent innervation pattern.

Furthermore, quantitative immunodetection studies carried out in our laboratory suggest that $\gamma$-MN axonal innervation of intrafusal fibers in the MS from Err $2 / 3^{c K O}$ was preserved. Moreover, sensory afferent responses recorded by using suction electrode recording technique in $E r r 2 / 3^{c K O}$ mice showed that the MSs were functional. This suggests that Err2 and Err3 do not seem to affect sensory and motor innervation patterns. Studies have shown that the combinatorial expression patterns of LIM-homeodomain (HD) transcription factors and Hox genes are responsible for the initial stages of MN diversification (Jessell, 2000; Dasen and Jessell, 2009; Philippidou and Dasen, 2013). However, the knowledge about how MNs maintain and tune their functions later in development is limited. Taken together, my studies showed that the expression of Err2 and Err3 transcription factors specifically promote $\gamma-\mathrm{MN}$ functional diversification but seem to have no influence on $\gamma$-MN identity, since the populations of small soma size putative $\gamma$-MNs with high FG levels and large soma size putative $\alpha-\mathrm{MNs}$ with low FG levels were both present in $E r r 2 / 3^{c K O}$ mice. Therefore, the high expression levels of Err2 and Err3 may endow certain cell populations with specific biophysical properties that in turn enable organisms to perform certain function. Hence, the high expression levels of Err2 and Err3 could potentially be used to classify particular cell type's biophysical properties and functions within a system.

\subsection{Outlook: The role of Err2 and Err3 in extraocular MN functional properties and proprioception}

To focus images on the fovea precisely, reflexive and voluntary eye movements are regulated by the extraocular muscles (EOMs), which possess proprioceptive receptors. The EOMs of submammalian species are devoid of muscle spindle (MS) proprioceptors, while mammalian species like humans, some primates, mice and the artiodactyls have them (see Donaldson, 2000; Büttner-Ennever, 2007 for review). Moreover, EOM tendons of artiodactyls have the Golgi tendon organ (GTOs) proprioceptors (Büttner-Ennever, 2007). Furthermore, a special putative proprioceptor called the palisade endings is present in the 
EOMs of nearly every species observed thus far (Büttner-Ennever, 2007). Even though these proprioceptors are observed in various species, a major gap in knowledge exists about whether these receptors generate sensory information that is provided to the nervous system and why a variation in the type of propriceptor exists between different species.

In mammals, there are six different types of extraocular muscles (EOMs) that form two groups: 1) recti EOMs (superior, inferior, medial and lateral) and 2) oblique EOMs (superior and inferior) (Spencer and Porter, 2006). While there are six morphologically and histochemically distinct EOM fiber types identified in mammals: Orbital layer containing 1) orbital singly innervated fiber type (orbital SIF), orbital multiply innervated fiber type (orbital MIF), and global layer containing 1) global red SIF, 2) global white SIF, 3) global intermediate SIF, 4) global MIF (Spencer and Porter, 2006; Büttner-Ennever, 2007). Moreover, studies have shown that EOM fiber types can be grouped into two types based on their morphology and physiological properties: non-twitch multiply innervated fiber types (MIF) and twitch singly innervated fiber types (SIF) (Spencer and Porter, 2006; BüttnerEnnever et al., 2001).

MNs from several nuclei including the oculomotor, trochlear and abudcens innervate EOM fibers that ultimately generate eye movements (Büttner-Ennever, 2007). Studies suggest that MIF or SIF innervating MNs in the oculomotor nucleus have different afferent inputs and thus, have different functions (Wasicky et al., 2004; Ugolini et al., 2006; BüttnerEnnever, 2007). Moreover, it is proposed that fast eye movements are produced by SIF innervating MNs, while MIF innervating MNs regulate muscle tension to produce smooth pursuit eye movements, convergence and gaze holding. Therefore, it would be interesting to investigate the differences in biophysical properties of these MN populations and how these properties influence eye movements in mice.

Since my current investigations in the spinal cord showed that Err2 and Err3 promote functional diversification of $\gamma$-MNs, I explored whether this mechanism may be relevant to the oculomotor system as well. Immunodetection studies I performed using midbrain slices from P21 Chat::Tomato transgenic mice expressing tdTomato in cholinergic neurons (Fig. 4.1D) showed that Err2 (Fig. 4.1A) and Err3 (Fig. 4.1B) are indeed expressed by oculomotor MNs. Moreover, previous studies have used retrograde tracers like cholera toxin subunit B (CTB) and Fluoro-Gold (FG) to characterize different MNs that innervate the extraocular muscles (Büttner-Ennever et al., 2002; Padmowijoyo and Yamadori, 1995). After characterizing oculomotor neurons with specific molecular markers, I plan to use retrograde tracers to label MNs and perform whole cell patch-clamp recordings of MNs from control 
mice initially to establish baseline properties of different $\mathrm{MN}$ types in the oculomotor nucleus. Furthermore, $\operatorname{Err} 2 / 3^{c K O}$ mice would be also injected with FG to label MNs in the oculomotor nucleus, recorded and compared to the control mice. In order to gauge whether the loss of Err2/3 in the oculomotor MNs has any impact on eye movements, both control and Err $2 / 3^{c K O}$ mice will be subjected to behavioral tests to observe fast eye movements versus smooth pursuit eye movements or gaze holding. Furthermore, stretch-responses from sensory afferents innervating superior, medial and inferior recti muscles and inferior oblique muscles that are innervated by oculomotor nuclei will be recorded, which may resolve the issue of whether these muscles can provide proprioceptive input to the nervous system and ultimately, influence eye movements. Furthermore, the afferent responses elicited due to the stretch of the same muscles can be performed in $E r r 2 / 3^{c K O}$ mice, which may show whether the lack of Err2/3 impacts the proprioceptor function. Therefore, the identification of a potential mechanism that drives oculomotor control may unravel the mystery of whether and to what extent extraocular muscles possess proprioceptive sense of eye movements. Ultimately, pursuing this line of investigation may reveal overlapping molecular strategies that tune neural biophysical properties to particular function throughout the nervous system.
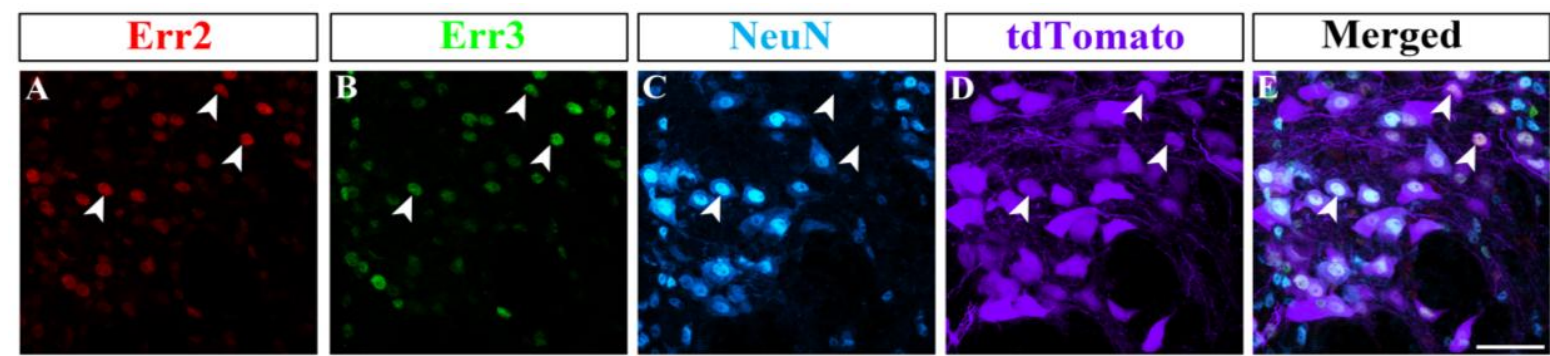

Figure 4.1 Err2 and Err3 expression in oculomotor nucleus of $\mathrm{ChAT}^{\mathrm{Cre}}$; Rosa26 ${ }^{\text {floxtdTomato }}$ mice at three weeks of age postnatally

(A-E) Err2 (red, nuclei), Err3 (green, nuclei), NeuN (blue, soma), tdTomato (purple, soma). Err2 and Err3 (arrows) are expressed in oculomotor MNs of the midbrain. The scale bar represents $50 \mu \mathrm{m}$. 


\section{SUMMARY}

In terrestrial vertebrates, the control of gait, posture and precision movements require a functioning muscle spindle (MS) system that is regulated by gamma motor neurons ( $\gamma$ MNs). $\gamma$-MNs regulate the MS sensitivity, however, another MN subtype called alpha motor neurons ( $\alpha$-MNs) generate muscle force. The molecular mechanisms that promote the functional diversification of MNs into $\alpha$ - and $\gamma$-subtypes remain largely unknown. The objective of this study was to characterize the role of novel transcription factors and their downstream targets in shaping $\gamma$-MN biophysical properties, and ultimately their role in determining gait, posture and precision movements in vivo.

Quantitative immunodetection studies showed that high levels of a known $\gamma-\mathrm{MN}$ marker, Err3, and a closely related protein, Err2, were co-expressed in putative $\gamma$-MNs that in addition had relatively small soma sizes, expressed low NeuN levels and incorporated high levels of Fluoro-Gold (FG) retrograde tracer. By contrast, putative $\alpha$-MNs expressed low levels of Err2 and Err3, expressed moderate-to-high levels of NeuN and incorporated low levels of FG.

To study MN biophysical properties and locomotion in mice lacking Err2 and Err3 expression specifically in their MNs, Err2 and Err3 conditional knockout $\left(E r r 2 / 3^{c K O}\right)$ mice were generated using the Cre/LoxP technology, which resulted in a successful deletion of Err2 and Err3 genes in MNs. The fortuitous finding that putative $\gamma$-MNs incorporate high levels of FG, conversely, $\alpha$-MNs incorporate low levels of FG provided the opportunity to study mature mouse $\gamma$-MN for the first time since studies in cat were performed in 1978. Indeed, whole cell patch-clamp studies using spinal cord slices from control mice showed that the $\mathrm{FG}^{\text {high }}$ putative $\gamma$-MNs had significantly different biophysical properties compared to the $\mathrm{FG}^{\text {low }}$ putative $\alpha$-MNs. After determining the baseline properties of $\alpha$-versus $\gamma$-MNs in control mice, recordings of the putative $\gamma$-MNs from $E r r 2 / 3^{c K O}$ mice showed shifted biophysical properties to putative $\alpha$-MN-like when compared to the putative $\gamma$-MNs from control mice. While no significant differences in biophysical properties were observed between the putative $\alpha$-MNs from $E r r 2 / 3^{c K O}$ mice versus the putative $\alpha$-MNs from control mice. Furthermore, the data showed that the loss of Err2/3 did not alter the Ia sensory afferent connectivity of the $\gamma$ - or $\alpha$-MNs from $\operatorname{Err} 2 / 3^{c K O}$ mice, therefore, implying that Err $2 / 3$ are important for determining $\gamma$-MN function but not identity.

Gait and posture in mice was studied using a high-speed video camera that captured locomotion from below while it moved across a transparent treadmill. The data showed a 
significant shift in gait and posture between $E r r 2 / 3^{c K O}$ versus control mice. Thus, the regulation of MS apparatus through functional $\gamma$-MN is essential for normal gait and posture during locomotion. Furthermore, precision movements were studied using a horizontal ladder and horizontal beam that the mice walked across, which showed significant differences in the precision movements of Err $2 / 3^{c K O}$ mice when compared to control mice. Thus, the lack of appropriate $\gamma$-MN biophysical properties to regulate MS apparatus function disrupts the execution of precision movements in Err $2 / 3^{c K O}$ mice.

Moreover, whole cell patch-clamp recordings in chick MNs that expressed Err2VP16 (fusion of heterologous transcriptional activation domain VP16 to Err2), showed shifted biophysical properties towards a $\gamma$-MN-like biophysical signature acquired through transcriptional activation. To investigate a potential mechanism through which Err2 may act, RNA-sequencing studies in chick spinal columns showed that the expression of Err2VP16 significantly up-regulated Kcnalo (a voltage-gated $\mathrm{K}^{+}$channel) gene expression. The bioinformatical analysis identified three putative Err2/3 binding sites in the evolutionary conserved region (ECR) of Kcna10, which was cloned into a DNA construct. Subsequently, co-electroporation experiments showed that Err2VP16 significantly elevated the tdTomato reporter gene activity that was under the control of Kcnalo ECR. Furthermore, the forced expression of Kcna10 protein in chick MNs shifted their biophysical properties towards a $\gamma$ MN-like biophysical signature. Overall, these studies suggest that Err2 may regulate $\gamma-\mathrm{MN}$ biophysical properties through regulating Kcna10 expression.

The major finding of this study is that Err2 and Err3 regulate $\gamma$-MN biophysical properties, which allow for the control of MS function that in turn provide proprioceptive information needed for control of gait, posture and precision movements during locomotion. Similar molecular strategies that drive functional diversification of neurons may operate throughout the nervous system. 


\section{REFERENCES}

Araki, T., and Terzuolo, C. A. (1962). Membrane currents in spinal motoneurons associated with the action potential and synaptic activity. J Neurophysiol. 25:772-89. pmid: 14013544

Adal, M. N., and Barker, D. (1965). Intramuscular branching of fusimotor fibres. J Physiol. 177:288-99. pmid: 14301026

Akay, T., Tourtellotte, W. G., Arber, S., Jessell, T. M. (2014). Degradation of mouse locomotor pattern in the absence of proprioceptive sensory feedback. Proc Natl Acad Sci U S A. 111(47):1687716882. doi: 10.1073/pnas.1419045111

Alaynick, W. A., Kondo, R. P., Xie, W., He, W., Dufour, C. R., Downes, M., Jonker, J. W., Giles. W., Naviaux, R. K., Giguère, V., Evans, R. M. (2007). ERR gamma directs and maintains the transition to oxidative metabolism in the postnatal heart. Cell Metab. 6(1):13-24. doi: 10.1016/j.cmet.2007.06.007

Amende, I., Kale, A., McCue, S., Glazier, S., Morgan, J. P., Hampton, T. G. (2005). Gait dynamics in mouse models of Parkinson's disease and Huntington's disease. J Neuroeng Rehabil. 2:20. doi:10.1186/1743-0003-2-20

Andrews S. (2010) FastQC: a quality control tool for high throughput sequence data. Available online at: http://www.bioinformatics.babraham.ac.uk/projects/fastqc

Arber, S., Ladle, D. R., Lin, J. H., Frank, E., Jessell, T. M. (2000). ETS gene Er81 controls the formation of functional connections between group Ia sensory afferents and motor neurons. Cell. 101(5):485-98. pmid: 10850491

Ashrafi, S., Lalancette-Hebert, M., Friese, A., Sigrist, M., Arber, S., Shneider, N. A. (2012). Wnt7A identifies embryonic gamma-motor neurons and reveals early postnatal dependence of gamma-motor neurons on a muscle spindle-derived signal. J. Neurosci. 32, 8725-8731. doi: 10.1523/JNEUROSCI.1160-12.2012

Banks R. W., Hulliger, M., Saed, H. H., Stacey, M. J. (2009). A comparative analysis of the encapsulated end-organs of mammalian skeletal muscles and of their sensory nerve endings. $J$ Anat. 214(6):859-87. doi: 10.1111/j.1469-7580.2009.01072.x.

Barker, D., Emonet-Dénand, F., Harker, D. W., Jami, L., Laporte, Y. (1977). Types of intra- and extrafusal muscle fibre innervated by dynamic skeleto-fusimotor axons in cat peroneus brevis and tenuissimus muscles, as determined by the glycogen-depletion method. J Physiol. 266(3):713-26. pmid: 140933

Barker, D. The morphology of muscle receptors. In: Handbook of Sensory Physiology Vol. III/2, Muscle Receptors. Ed. Hunt, C.C. Springer-Verlag, Berlin-Heidelberg-New York, 1974.

Barrett, E. F., Barrett, J. N., and Crill, W. E. (1980). Voltage-sensitive outward currents in cat motor neurones. J Physiol. 304:251-76. pmid: 7441537

Beato, M., Sa'nchez-Pacheco, A. (1996). Interaction of steroid hormone receptors with the transcription initiation complex. Endocr Rev. 17(6):587-609. doi: 10.1210/edrv-17-6-587 
Bellardita, C., and Kiehn, O. (2015). Phenotypic characterization of speed-associated gait changes in mice reveals modular organization of locomotor networks. Curr Biol. 25(11):1426-36. doi: 10.1016/j.cub.2015.04.005.

Bel-Vialar, S., Itasaki, N., and Krumlauf, R. (2002). Initiating Hox gene expression: in the early chick neural tube differential sensitivity to FGF and RA signalling subdivides the HoxB genes in two distinct groups. Development. 129(22):5103-15. pmid: 12399303

Benoit, G., Cooney, A., Giguere, V., Ingraham, H., Lazar, M., Muscat, G., Perlmann, T., Renaud, J.P., Schwabe, J., Sladek, F., Tsai, M.J., Laudet, V. (2006). International Union of Pharmacology. LXVI. Orphan nuclear receptors. Pharmacol Rev. 58(4):798-836. doi: $10.1124 /$ pr.58.4.10

Binder, M. D., Heckman, C. J., and Powers, R. K. (2011). The physiological control of motor neuron activity. In: Comprehensive Physiology. John Wiley \& Sons, Inc. https://dx.doi.org/10.1002/cphy.cp120101

Bourane, S., Grossmann, K. S., Britz, O., Dalet, A., Del Barrio, M. G., Stam, F. J., GarciaCampmany, L., Koch, S., Goulding, M. (2015). Identification of a spinal circuit for light touch and fine motor control. Cell. 160(3):503-515. doi:10.1016/j.cell.2015.01.011

Burke, R. Spinal cord: Ventral horn. In: The Synaptic Organization of the Brain, Ed. Shepherd GM. Oxford Univ Press, New York, 2004, p. 79-123.

Burke, R. E. Motor Units: Anatomy, Physiology, and Functional Organization. In: Handbook of Physiology, The Nervous System, Motor Control. John Wiley \& Sons, Inc. 2011. doi: 10.1002/cphy.cp010210

Burke, R. E., and Rudomin, P. Spinal nervous and synapses. In: Handbook of Physiology. The Nervous System. Cellular Biology of Neurons. John Wiley \& Sons, Inc. 2011. doi: 10.1002/cphy.cp010124

Burke, R. E., Levine, D. N., Tsairis, P., Zajac, F. E. (1973). Physiological types and histochemical profiles in motor units of the cat gastrocnemius. J Physiol. 234(3):723-48. pmid: 4148752

Burke, R. E., Strick, P. L., Kanda, K., Kim, C. C., Walmsley, B. (1977). Anatomy of medial gastrocnemius and soleus motor nuclei in cat spinal cord. J Neurophysiol. 40(3):667-80. pmid: 874534

Büttner-Ennever, J. A. Anatomy of the Oculomotor SystemStraube A, Büttner U (eds): NeuroOphthalmology. Dev Ophthalmol. Basel, Karger, 2007, vol 40, pp 1-14.

Büttner-Ennever, J. A., Horn, A. K., Graf, W., Ugolini, G. (2002). Modern concepts of brainstem anatomy: from extraocular motoneurons to proprioceptive pathways. Ann N Y Acad Sci. 956:75-84. pmid: 11960795

Büttner-Ennever, J. A., Horn, A. K., Scherberger, H., D'Ascanio, P. (2001). Motoneurons of twitch and nontwitch extraocular muscle fibers in the abducens, trochlear, and oculomotor nuclei of monkeys. J Comp Neurol. 438(3):318-35. pmid: 11550175

Briscoe, J., Pierani, A., Jessell, T. M., Ericson, J. (2000). A homeodomain protein code specifies progenitor cell identity and neuronal fate in the ventral neural tube. Cell. 101(4):435-45. pmid: 10830170

Carp, J. S. (1992). Physiological properties of primate lumbar rnotoneurons. J Neurophysiol. 68(4):1121-32. pmid: 1432072 
Catela, C., Shin, M. M., and Dasen, J. S. (2015). Assembly and function of spinal circuits for motor control. Annu Rev Cell Dev Biol. 31:669-98. doi: 10.1146/annurev-cellbio-100814-125155. Epub 2015 Sep 21.

Chakkalakal, J. V., Nishimune, H., Ruas, J. L., Spiegelman, B. M., Sanes, J. R. (2010). Retrograde influence of muscle fibers on their innervation revealed by a novel marker for slow motor neurons. Development. 137(20):3489-99. doi: 10.1242/ dev.053348

Chal J, and Pourquié O. (2017). Making muscle: skeletal myogenesis in vivo and in vitro. Development. 144(12):2104-2122. doi: 10.1242/dev.151035.

Chen, J. D., and Evans, R. M. (1995). A transcriptional co-repressor that interacts with nuclear hormone receptors. Nature. 377(6548):454-7. doi: 10.1038/377454a0

Chen, J., and Nathans, J. (2007). Estrogen-related receptor beta/NR3B2 controls epithelial cell fate and endolymph production by the stria vascularis. Dev Cell. 13(3):325-37. doi: 10.1016/j.devcel.2007.07.011

Chen, H. H., and Frank, E. (1999). Development and specification of muscle sensory neurons. Curr Opin Neurobiol. 9(4):405-9.

Cody, F. W., Harrison, L. M., Taylor, A. (1975). Analysis of activity of muscle spindles of the jawclosing muscles during normal movements in the cat. J Physiol. 253(2):565-82.

Collin, R. W., Kalay, E., Tariq, M., Peters, T., van der Zwaag, B., Venselaar, H., Oostrik, J., Lee, K., Ahmed, Z. M., Caylan, R., Li, Y., Spierenburg, H.A., Eyupoglu, E., Heister, A., Riazuddin, S., Bahat, E., Ansar, M., Arslan, S., Wollnik, B., Brunner, H. G., Cremers, C. W., Karaguzel, A., Ahmad, W., Cremers, F. P., Vriend, G., Friedman, T. B., Riazuddin, S., Leal, S. M., Kremer, H. (2008). Mutations of ESRRB encoding estrogen-related receptor beta cause autosomal-recessive nonsyndromic hearing impairment DFNB35. Am J Hum Genet. 82(1):125-38. doi: 10.1016/j.ajhg.2007.09.008

Cooper, S. (1961). The responses of primary and secondary endings of muscle spindles with intact motor innervation during applied stretch. Q J Exp Physiol Cogn Med Sci. 46:389-98. pmid: 13881160

Corda, M., von Euler, C., and Lennerstrand, G. (1966). Reflex and cerebellar influences on alpha and on "rhythmic" and "tonic" gamma activity in the intercostal muscle. J Physiol. 184(4):898-923. pmid: 4223683

Crill, W E. (1996). Persistent sodium current in mammalian central neurons. Annu Rev Physiol. 58:349-62. doi: 10.1146/annurev.ph.58.030196.002025

Dasen, J. S. and Jessell, T. M. (2009). Hox networks and the origins of motor neuron diversity. Curr.Top.Dev.Biol. 88, 169-200. doi: 10.1016/S0070- 2153(09)88006-X

Dasen, J. S, Liu, J. P., and Jessell, T. M. (2003). Motor neuron columnar fate imposed by sequential phases of Hox-c activity. Nature 425:926-33. pmid: 14586461 doi: 10.1038/nature02051

Delgado-García, J. M. (2015). Cajal and the Conceptual Weakness of Neural Sciences. Front Neuroanat. 9:128. doi:10.3389/fnana.2015.00128

Digigait $^{\text {TM }}$ (2011). Mouse Specifics, Inc. MA, USA.

Dietrichson, P. (1971). Tonic ankle reflex in parkinsonian rigidity and in spasticity. The role of the fusimotor system. Acta Neurol Scand. 47(2):163-82. pmid: 5566567 
Donaldson, I. M. (2000). The functions of the proprioceptors of the eye muscles.Philos Trans R Soc Lond B Biol Sci. 2000 Dec 29;355(1404):1685-754. doi: 10.1098/rstb.2000.0732

Dufour, C. R., Wilson, B. J., Huss, J. M., Kelly, D. P., Alaynick, W. A., Downes, M., Evans, R. M., Blanchette, M., Giguère, V. (2007). Genome-wide orchestration of cardiac functions by the orphan nuclear receptors ERRalpha and gamma. Cell Metab. 5(5):345-56. doi: 10.1016/j.cmet.2007.03.007

Duboule, D. and Dolle, P. (1989). The structural and functional organization of the murine HOX gene family resembles that of Drosophila homeotic genes. EMBO J. 8(5):1497-505. pmid: 2569969

Durand, J., Filipchuk, A., Pambo-Pambo, A., Amendola, J., Borisovna Kulagina, I., Guéritaud, J. P. (2015). Developing electrical properties of postnatal mouse lumbar motor neurons. Front Cell Neurosci. 9:349. doi: 10.3389/fncel.2015.00349

Duysens, J., and Pearson, K. G. (1980). Inhibition of flexor burst generation by loading ankle extensor muscles in walking cats. Brain Res. 187(2):321-332. pmid: 7370733

Eccles, J. C. (1955). The central action of antidromic impulses in motor nerve fibres. Pflugers. Arch. ges.Physiol. 260(5):385-415. pmid: 13245371

Eccles, J. C., Eccles, R. M., and Lundberg, A. (1957a). Durations of after-hyperpolarization of motoneurones supplying fast and slow muscles. Nature. 179(4565):866-8. pmid: 13430719

Eccles, J. C., Eccles, R. M., and Lundberg, A. (1957b). The convergence of monosynaptic excitatory afferents on to many different species of alpha motoneurones. J Physiol. 137(1):22-50. pmid: 13439582

Eccles, J. C., Eccles, R. M., Iggo, A., Lundberg, A. (1960). Electrophysiological studies on gamma motor neurones. Acta Physiol Scand 50:32- 40. pmid: 13725574

Edwards, I. J., Bruce, G., Lawrenson, C., Howe, L., Clapcote, S. J., Deuchars, S. A., Deuchars, J. (2013). Na+/K+ ATPase alpha1 and alpha3 isoforms are differentially expressed in alpha- and gamma-motor neurons. J. Neurosci. 33, 9913-9919. doi: 10.1523/ JNEUROSCI.5584-12.2013

Enjin, A., Leão, K. E., Mikulovic, S., Le Merre, P., Tourtellotte, W. G., Kullander, K. (2012). Sensorimotor function is modulated by the serotonin receptor $1 \mathrm{~d}$, a novel marker for gamma motor neurons. Mol Cell Neurosci. 49(3):322-32. doi: 10.1016/ j.mcn.2012.01.003

Enjin, A., Rabe, N., Nakanishi, S.T., Vallstedt, A., Gezelius, H., Memic, F., Lind, M., Hjalt, T., Tourtellotte, W. G., Bruder, C., Eichele, G., Whelan, P. J., Kullander, K. (2010). Identification of novel spinal cholinergic genetic subtypes disclose Chodl and Pitx2 as markers for fast motor neurons and partition cells. J Comp Neurol. 518(12):2284-2304. doi:10.1002/cne.22332.

Ericson, J., Rashbass, P., Schedl, A., Brenner-Morton, S., Kawakami, A., van Heyningen, V., Jessell, T. M., Briscoe, J. (1997a). Pax6 controls progenitor cell identity and neural fate in response to graded Shh signaling. Cell. 90(1):169-80. pmid: 9230312

Ericson, J., Briscoe, J., Rashbass, P., van Heyningen, V., Jessell, T. M. (1997b). Graded sonic hedgehog signaling and the specification of cell fate in the ventral neural tube. Cold Spring Harb Symp Quant Biol. 62:451-66. pmid:9598380

Ernfors, P., Lee, K. F., Kucera, J., Jaenisch, R. (1994). Lack of neurotrophin-3 leads to deficiencies in the peripheral nervous system and loss of limb proprioceptive afferents. Cell. 77(4):503-12. pmid: 7514502 
Falgairolle, M., and O'Donovan, M. J. (2015). Pharmacological Investigation of Fluoro-Gold Entry into Spinal Neurons. PLoS One. 10(6):e0131430. doi: 10.1371/journal.pone.0131430. eCollection 2015.

Feldman, A. G., Orlovsky, G. N., and Perret, C. (1977). Activity of muscle spindle afferents during scratching in the cat. Brain Res. 129(1):192-6. pmid: 141319

Fitz-Ritson, D. (1982). The anatomy and physiology of the muscle spindle, and its role in posture and movement: a review. J Can Chiropr Assoc. 26(4): 144-150.

Fleshman, J. W., Munson, J. B., Sypert, G. W., Friedman, W. A. (1981). Rheobase, input resistance, and motor unit type in medial gastrocnemius motoneurons in the cat. $J$ Neurophysiol. 46(6):1326-38. pmid: 6275043

Fogarty, M. J., Hammond, L. A., Kanjhan, R., Bellingham, M. C., Noakes, P. G. (2013). A method for the three-dimensional reconstruction of Neurobiotin ${ }^{\mathrm{TM}}$-filled neurons and the location of their synaptic inputs. Front Neural Circuits. 7:153. doi: 10.3389/fncir.2013.00153

Freedman, L. P. (1999). Increasing the complexity of coactivation in nuclear receptor signaling. Cell. 97(1):5-8. pmid: 10199396

Friese, A., Kaltschmidt, J. A., Ladle, D. R., Sigrist, M., Jessell, T. M., Arber, S. (2009). Gamma and alpha motor neurons distinguished by expression of transcription factor Err3. Proc Natl Acad Sci U S A. 106(32):13588-93. doi: 10.1073/pnas.0906809106

Gardiner, P. F. (1993). Physiological properties of motoneurons innervating different muscle unit types in rat gastrocnemius. J Neurophysiol. 69(4):1160-70. doi: 10.1152/jn.1993.69.4.1160

Gaunt, S. J. (1988). Mouse homeobox gene transcripts occupy different but overlapping domains in embryonic germ layers and organs: a comparison of Hox-3.1 and Hox-1.5. Development. 103(1):13544. pmid: 2904354

Giguère, V. (1999). Orphan nuclear receptors: from gene to function. Endocr Rev. 20(5):689-725. doi: 10.1210/edrv.20.5.0378

Giguère, V. (2002). To ERR in the estrogen pathway. Trends Endocrinol Metab. 13(5):220-5. pmid: 12185669

Glass, C.K. (1994). Differential recognition of target genes by nuclear receptors monomers, dimers, and heterodimers. Endocr Rev. 15(3):391-407. doi: 10.1210/edrv-15-3-391

Glass, C. K., Rose, D. W., and Rosenfeld, M. G. (1997). Nuclear receptor coactivators. Curr Opin Cell Biol. 9(2):222-32. pmid: 9069256

Glover, J. C., Renaud, J. S., and Rijli, M. (2006). Retinoic acid and hindbrain patterning. $J$ Neurobiol. 66(7):705-25. doi: 10.1002/neu.20272

Gordon, T., Thomas, C. K., Munson, J. B., Stein, R. B. (2004). The resilience of the size principle in the organization of motor unit properties in normal and reinnervated adult skeletal muscles. Can $J$ Physiol Pharmacol. 82(8-9):645-61. doi: 10.1139/y04-081

Graham, A., Papalopulu, N., and Krumlauf, R. (1989). The murine and Drosophila homeobox clusters have common features of organization and expression. Cell. 57(3):367-78. pmid: 2566383

Granit, R. The Basis of Motor Control. London and New York: Academic Press, 1970. 
Grapov, D. (2014). DeviumWeb: Dynamic Multivariate Data Analysis and Visualization Platform. v0.3.2 (25/11/14). doi :10.5281/zenodo. 12879

Grantyn, R., and Grantyn, A. (1978). Morphological and electrophysiological properties of cat abducens motoneurons. Exp. Brain Res. 31(2): 249-274. pmid: 631242

Grigg, P., Finerman, G. A., and Riley, L. H. (1973). Joint-position sense after total hip replacement. J Bone Joint Surg Am 55(5): 1016-1025. pmid: 4760087

Grillner, S. (2003). The motor infrastructure: from ion channels to neuronal networks. Nat Rev Neurosci. 4(7):573-86. doi: 10.1038/nrn1137

Grillner, S. (2006). Biological pattern generation: the cellular and computational logic of networks in motion. Neuron. 52(5):751-66. doi: 10.1016/j.neuron.2006.11.008

Grillner, S., and Rossignol, S. (1978). On the initiation of the swing phase of locomotion in chronic spinal cats. Brain Res. 146(2):269-277. pmid: 11535680

Gustafsson, B., and Pinter, M. J. (1984). An investigation of threshold properties among cat spinal alpha-motoneurones. J Physiol. 357:453-83. pmid: 6512700

Gutman, C., Ajmera, M. K., Hollyday, M. (1993). Organization of motor pools supplying axial muscles in the chicken. Brain Res. 609,129-1. pmid: 8508296

Hadzipasic, M., Tahvildari, B., Nagy, M., Bian, M., Horwich, A.L., McCormick, D. A. (2014). Selective degeneration of a physiological subtype of spinal motor neuron in mice with SOD1-linked ALS. Proc Natl Acad Sci USA 111(47), 16883-8. doi: 10.1073/pnas.1419497111

Hagbarth, K. E., Wallin, G., Löfstedt, L., Aquilonius, S. M. (1975). Muscle spindle activity in alternating tremor of Parkinsonism and in clonus. J Neurol Neurosurg Psychiatry. 38(7):636-41. pmid: 125783

Halder, R., Hennion, M., Vidal, R. O., Shomroni, O., Rahman, R. U., Rajput, A., Centeno, T. P., van Bebber, F., Capece, V., Garcia Vizcaino, J. C., Schuetz, A. L., Burkhardt, S., Benito, E., Navarro Sala, M., Javan, S. B., Haass, C., Schmid, B., Fischer, A., Bonn, S. (2016). DNA methylation changes in plasticity genes accompany the formation and maintenance of memory. Nature Neuroscience 19(1), 102-110. doi: 10.1038/nn.4194

Hall, B., Limaye, A., and Kulkarni, A. B. (2009). Overview: generation of gene knockout mice. Curr Protoc Cell Biol. Chapter 19:Unit 19.12 19.12.1-17. doi: 10.1002/0471143030.cb1912s44.

Han, K., and Manley, J. L. (1993). Functional domains of the Drosophila Engrailed protein. EMBO J. 12(7):2723-33. pmid: 8334991

Henneman, E., Somjen, G., and Carpenter, D. O. (1965a). Functional significance of cell size in spinal motoneurons. J Neurophysiol. 28:560-80. pmid: 14328454

Henneman, E., Somjen, G., and Carpenter, D, O. (1965b). Excitability and inhibitability of motoneurons of different sizes. J Neurophysiol. 28(3):599-620. pmid: 5835487

Haase, G., Dessaud, E., Garces, A., de Bovis, B., Birling, M., Filippi, P., Schmalbruch, H., Arber, S., and deLapeyriere, $\mathbf{O}$. (2002). GDNF acts through PEA3 to regulate cell body positioning and muscle innervation of specific motor neuron pools. Neuron 35(5), 893-905. pmid: 12372284 
Heckman, C. J., and Enoka, R. M. (2012). Motor unit. Compr Physiol. 2(4):2629-82. doi: 10.1002/cphy.c100087.

Heckman, C. J., and Enoka, R. M. (2004). Physiology of the motor neuron and the motor unit. In: Clinical Neurophysiology of Motor neuron Diseases Handbook of Clinical Neurophysiology, Vol. 4. Elsevier B.V. https://doi.org/10.1016/S1567-4231(04)04006-7

Heemskerk J, DiNardo S, Kostriken R, O'Farrell P. H. (1991) Multiple modes of engrailed regulation in the progression towards cell fate determination. Nature. 352(6334):404-10. doi: $10.1038 / 352404 \mathrm{a} 0$

Hiebert, G. W., Whelan, P. J., Prochazka, A., Pearson, K. G. (1996). Contribution of hind limb flexor muscle afferents to the timing of phase transitions in the cat step cycle. $J$ Neurophysiol. 75(3):1126-1137. pmid: 8867123

Hollyday, M. (1980). Organization of motor pools in the chick lumbar lateral motor column. J Comp Neurol. 194(1):143-70. doi: 10.1002/cne.901940108

Hong, H., Yang, L., and Stallcup, M. R. (1999). Hormone-independent transcriptional activation and coactivator binding by novel orphan nuclear receptor ERR3. J Biol Chem. 274(32):22618-26. pmid: 10428842

Horlein, A. J., Naar, A. M., Heinzel, T., Torchia, J., Gloss, B., Kurokawa, R., Ryan, A., Kamel, Y., Soderstrom, M., Glass, C. K., Rosenfeld, M. G. (1995). Ligand-independent repression by the thyroid hormone receptor mediated by a nuclear receptor co-repressor. Nature. 377(6548):397-404. doi: $10.1038 / 377397 \mathrm{a} 0$

Horwitz, K. B., Jackson, T. A., Bain, D.L., Richer, J. K., Takimoto, G. S., Tung, L. (1996). Nuclear receptor coactivators and corepressors. Mol Endocrinol. 10(10):1167-77. doi: 10.1210/mend.10.10.9121485

Houk, J., and Henneman, E. (1967). Responses of Golgi tendon organs to active contractions of the soleus muscle of the cat. J Neurophysiol. 30(3):466-81. doi: 10.1152/jn.1967.30.3.466

Hulliger, M. (1984). The mammalian muscle spindle and its central control. Rev Physiol Biochem Pharmacol. 101:1-110. pmid: 6240757

Humphries, E. S., and Dart, C. (2015). Neural and Cardiovascular Potassium Channels as Therapeutic Drug Targets: Promise and Pitfalls. J Biomol Screen. 20(9):1055-73. doi: $10.1177 / 1087057115601677$

Hunt, C. C. (1951). The reflex activity of mammalian small-nerve fibres. $J$ Physiol. 115(4):456-69. pmid: 14898522

Hunt, C. C., and Kuffler, S. W. (1951a). Further study of efferent small-nerve fibers to mammalian muscle spindles; multiple spindle innervation and activity during contraction. $J$ Physiol. 113(2-3):28397. pmid: 14832775

Hunt, C. C., and Kuffler, S. W. (195lb). Stretch receptor discharges during muscle contraction. $J$ Physiol. 113(2-3):298-315. pmid: 14832776

Hunt, C. C., and Ottoson, D. (1976). Initial burst of primary endings of isolated mammalian muscle spindles. J Neurophysiol. 39(2):324-30. doi: 10.1152/jn.1976.39.2.324

Hunt, C. C., and Paintal, A. S. (1958). Spinal reflex regulation of fusimotor neurones. J Physiol. 143(2):195-212. pmid: 13588549 
Huss, J. M., Imahashi, K., Dufour, C. R., Weinheimer, C. J., Courtois, M., Kovacs, A., Giguère, V., Murphy, E., Kelly, D. P. (2007). The nuclear receptor ERRalpha is required for the bioenergetic and functional adaptation to cardiac pressure overload. Cell Metab. 6(1):25-37. doi: 10.1016/j.cmet.2007.06.005

Huss, J. M., and Kelly, D. P. (2004). Nuclear receptor signaling and cardiac energetics. Circ Res. 95(6):568-78. doi: 10.1161/01.RES.0000141774.29937.e3

Hippenmeyer, S., Shneider, N. A., Birchmeier, C., Burden, S. J., Jessell, T. M., Arber, S. (2002). A role for neuregulin1 signaling in muscle spindle differentiation. Neuron. 36(6):1035-49. pmid: 12495620

Inoue, H., Nojima, H., and Okayama, H. (1990). High efficiency transformation of Escherichia coli with plasmids. Gene. 96(1):23-8. pmid: 2265755

Institut Clinique de la Souris (ICS), Alsace, France. http://www.ics-mci.fr/en/resources-andtechnologies/nuclear-receptor-zoo/

Iwasa, H., Kurabayashi, M., Nagai, R., Nakamura, Y., Tanaka, T. (2001). Genetic variations in five genes involved in the excitement of cardiomyocytes. J Hum Genet. 46(9):549-52. doi: $10.1007 / \mathrm{s} 100380170039$

Jami, L. (1992). Golgi tendon organs in mammalian skeletal muscle: functional properties and central actions. Physiol Rev. 72(3):623-66. pmid: 162603

Jessell, T. M. (2000). Neural specification in the spinal cord: inductive signals and transcriptional codes. Nat.Rev.Genet. 1, 20-29. doi: 10.1038/35049541

Jiang, Z., Carlin, K. P., and Brownstone, R. M. (1999). An in vitro functionally mature mouse spinal cord preparation for the study of spinal motor networks. Brain Res 816: 493-499.

Jung, H., Lacombe, J., Mazzoni, E. O., Liem, Jr. K. F., Grinstein, J., Mahony, S., Mukhopadhyay, D., Gifford, D. K., Young, R. A., Anderson, K. V., Wichterle, H., Dasen, J. S. (2010). Global control of motor neuron topography mediated by the repressive actions of a single Hox gene. Neuron. 67(5):781-96. doi: 10.1016/j.neuron.2010.08.008

Kandel, E. R., Schwartz, J. H., and Jessell, T. M. (2000). Principles of neural science (4th ed.). New York: McGraw-Hill, Health Professions Division. 2000.

Kanning, K. C., Kaplan, A., and Henderson, C. E. (2010). Motor neuron diversity in development and disease. Annu Rev Neurosci. 33:409-40. doi: 10.1146/annurev .neuro.051508.135722

Kaplan, A., Spiller, K. J., Towne, C., Kanning, K. C., Choe, G. T., Geber, A., Akay, T., Aebischer, P., Henderson, C. E. (2014). Neural matrix metalloproteinase-9 is a determinant of selective neurodegeneration. Neuron. 81(2):333-48. doi: 10.1016/j.neuron.2013.12.009

Kawakami, K. (2007). Tol2: a versatile gene transfer vector in vertebrates. Genome Biol. 8, Suppl 1:S7 (Review). doi:10.1186/gb-2007-8-s1-s7

Kawakami, and K., Shima, A. (1999). Identification of the Tol2 transposase of the medaka fish Oryzias latipes that catalyzes excision of a nonautonomous Tol2 element in zebrafish Danio rerio. Gene. 15;240(1):239-244. pmid: 10564832

Kemm, R. E., and Westbury, D. R. (1978). Some properties of spinal gamma-motor neurones in the cat, determined by micro-electrode recording. J Physiol. 282:59-71. pmid: 722563 
Kernell, D. (1965a). The adaptation and the relation between discharge frequency and current strength of cat lumbosacral motoneurones stimulated by long-lasting injected currents. Acta Physiol. Scand. 65: 65-73. doi: 10.1111/j.1748-1716.1965.tb04250.x

Kernell, D. (1965b). High frequency repetitive firing of cat lumbosacral motoneurones stimulated by long-lasting injected currents. Acta Physiol. Scand. 65: 74-86. doi: 10.1111/j.17481716.1965.tb04251.x

Kiehn, O. (2016). Decoding the organization of spinal circuits that control locomotion. Nat Rev Neurosci. 17(4):224-38. doi: 10.1038/nrn.2016.9.

Kiehn, O. (2006). Locomotor circuits in the mammalian spinal cord. Annu Rev Neurosci. 29:279-306. doi: 10.1146/annurev.neuro.29.051605.112910

Klein, R., Silos-Santiago, I., Smeyne, R.J., Lira, S.A., Brambilla, R., Bryant, S., Zhang, L., Snider, W. D., Barbacid, M. (1994). Disruption of the neurotrophin-3 receptor gene trkC eliminates la muscle afferents and results in abnormal movements. Nature. 368(6468):249-51. pmid: 8145824

Kobayashi, Y., Oshim, K., and Tasaki, I. (1952). Analysis of afferent and efferent systems in the muscle nerve of the toad and cat. J Physiol. 117(2):152-71. pmid: 14955806

Kucera, J., and Walro, J. M. (1992). Formation of muscle spindles in the absence of motor innervation. Neurosci Lett. 145(1):47-50. pmid: 1461566

Kucera, J., Walro, J. M., and Reichler, J. (1988) Innervation of developing intrafusal muscle fibers in the rat. Am J Anat. 183(4):344-58. pmid: 3218622

Kuffler, S. W., Hunt, C. C., Quilliam, J. P. (1951). Function of medullated small-nerve fibers in mammalian ventral roots; efferent muscle spindle innervation. J Neurophysiol. 14(1):29-54. pmid: 14784872

Lacombe, J., Hanley, O., Jung, H., Philippidou, P., Surmeli, G., Grinstein, J., Dasen, J. S. (2013). Genetic and functional modularity of Hox activities in the specification of limb-innervating motor neurons. PLOS Genet. 9:e1003184. doi: 10.1371/journal.,pgen.1003184

Ladle, D. R., Pecho-Vrieseling, E., Arber, S. (2009). Assembly of motor circuits in the spinal cord: driven to function by genetic and experience-dependent mechanisms. Neuron. 56(2):270-83.

Lam, T., Pearson, K. G. (2001). Proprioceptive modulation of hip flexor activity during the swing phase of locomotion in decerebrate cats. J Neurophysiol. 86(3):1321-1332. pmid: 11535680

Landmesser, L. (1978a.). The distribution of motoneurones supplying chick hind limb muscles. $J$. Physiol. 284, 371-389. pmid: 731549

Landmesser, L. (1978b). The development of motor projection patterns in the chick hind limb. $J$. Physiol. 284, 391-414. pmid: 731552

Landmesser, L. (1980). The generation of neuromuscular specificity. Annu. Rev. Neurosci. 3, 279302.

Landmesser, L. (2001). The acquisition of motoneuron subtype identity and motor circuit formation. Int. J. Dev. Neurosci. 19, 175-182. 
Lang, R., Lee, G., Liu, W., Tian, S., Rafi, H., Orias, M., Segal, A. S., Desir, G. V. (2000). KCNA10: a novel ion channel functionally related to both voltage-gated potassium and CNG cation channels. Am J Physiol Renal Physiol. 278(6):F1013-21. dio: 10.1152/ajprenal.2000.278.6.F1013

Langmead, B., and Salzberg, S. L. (2012). Fast gapped-read alignment with Bowtie 2. Nat. Methods 9(4), 357-359. doi: 10.1038/nmeth.1923

Laporte, Y., Emonet-Dénand, F., Jami, L. (1981). The skeletofusimotor or/ $\beta$-innervation of mammalian muscle spindles. TINS 4:97-99. https://doi.org/10.1016/0166-2236(81)90031-X

Leber, S. M., Breedlove, S. M., and Sanes, J. R. (1990). Lineage, arrangement, and death of clonally related motoneurons in chick spinal cord. J Neurosci. 10(7):2451-62. pmid: 2376781

Lee, T. (2013). Tsung-I Lee "Genetic factors driving the functional specification of spinal motor neurons," Ph.D. Dissertation. Georg-August-Universität in Göttingen, 2013 dc.identifier.urn (urn:nbn:de:gbv:7-webdoc-3777-6).

Lee, S. I., Conrad, T., Jones, S. M., Lagziel, A., Starost, M. F., Belyantseva, I. A., Friedman, T. B., Morell, R. J. (2013). A null mutation of mouse Kcna10 causes significant vestibular and mild hearing dysfunction. Hear Res. 300:1-9. doi: 10.1016/j.heares.2013.02.009.

Leksell, L. (1945). The action potential and excitatory effects of the small ventral root fibres to skeletal muscle. Acta Physiol Scand (Suppt 31)10:1-84.

Leroy, F., Lamotte d'Incamps, B., and Zytnicki, D. (2015). Potassium currents dynamically set the recruitment and firing properties of F-type motoneurons in neonatal mice. $J$ Neurophysiol. 114(3):1963-73. doi: 10.1152/jn.00193.2015

Li, B., and Dewey, C. N. (2011). RSEM: accurate transcript quantification from RNA-Seq data with or without a reference genome. BMC Bioinformatics 12, 223. doi: 10.1186/1471-2105-12-323

Li, S., Zhuang, C., Hao, M., He, X., Marquez, J. C., Niu, C. M., Lan, N. (2015). Coordinated alpha and gamma control of muscles and spindles in movement and posture. Front Comput Neurosci. 9:122. doi: 10.3389/fncom.2015.00122.

Lin, J. H., Saito, T., Anderson, D. J., Lance-Jones, C., Jessell, T. M., Arber, S. (1998). Functionally related motor neuron pool and muscle sensory afferent subtypes defined by coordinate ETS gene expression. Cell. 95(3), 393-407. pmid: 9814709

Liu, J. P., Laufer, E., and Jessell, T. M. (2001) Assigning the positional identity of spinal motor neurons: rostrocaudal patterning of Hox-c expression by FGFs, Gdf11, and retinoids. Neuron. 32(6):997-1012. pmid: 11754833

Livet, J., Sigrist, M., Stroebel, S., De Paola, V., Price, S. R., Henderson, C. E., Jessell, T. M., Arber, S. (2002). ETS gene Pea3 controls the central position and terminal arborization of specific motor neuron pools. Neuron. 35:877-892. pmid: 12372283

Love, M. I., Huber, W., and Anders, S. (2014). Moderated estimation of fold change and dispersion for RNA-seq data with DESeq2. Genome Biol. 15 (12), 550. doi: 10.1186/s13059-014-0550-8

Lu, D. C., Niu, T., Alaynick, W. A. (2015). Molecular and cellular development of spinal cord locomotor circuitry. Front Mol Neurosci. 8:25. doi: 10.3389/fnmol.2015.00025

Luo, J., Sladek, R., Bader, J. A., Matthyssen, A., Rossant, J., Giguère, V. (1997). Placental abnormalities in mouse embryos lacking the orphan nuclear receptor ERR-beta. Nature. 388(6644):778-82. doi: 10.1038/42022 
Luo, J., Sladek, R., Carrier, J., Bader, J. A., Richard, D., Giguère, V. (2003). Reduced fat mass in mice lacking orphan nuclear receptor estrogen-related receptor alpha. Mol Cell Biol. 23(22):7947-56. pmid: 14585956

Madisen, L., Zwingman, T. A., Sunkin, S. M., Oh, S. W., Zariwala, H. A., Gu, H., Ng, L.L., Palmiter, R. D., Hawrylycz, M. J., Jones, A. R., Lein, E. S., Zeng, H. (2010). A robust and highthroughput Cre reporting and characterization system for the whole mouse brain. Nature Neuroscience. 13(1),133-140. doi: 10.1038/nn.2467

Manuel, M., and Zytnicki, D. (2011). Alpha, beta and gamma motoneurons: functional diversity in the motor system's final pathway. $J$ Integr Neurosci. 10(3):243-76. doi: $10.1142 / \mathrm{S} 0219635211002786$

Manuel, M., Iglesias, C., Donnet, M., Leroy, F., Heckman, C. J., Zytnicki, D. (2009). Fast kinetics, high-frequency oscillations, and sub.p.rimary firing range in adult mouse spinal motor neurons. J Neurosci. 29(36), 11246-11256. doi: 10.1523/JNEUROSCI.3260-09.2009

Marquardt, T., and Pfaff, S. L. (2001). Cracking the transcriptional code for cell specification in the neural tube. Cell. 106(6):651-4. pmid: 11572771

Masland, R. H. (2004). Neural cell types. Curr Biol. 14(13):R497-500. doi: 10.1016/j.cub.2004.06.035

Mathelier, A., Fornes, O., Arenillas, D. J., Chen, C.Y., Denay, G., Lee, J., Shi, W., Shyr, C., Tan, G., Worsley-Hunt, R., Zhang, A. W., Parcy, F., Lenhard, B, Sandelin, A., Wasserman, W. W. (2016). JASPAR 2016: a major expansion and update of the open-access database of transcription factor binding profiles. Nucleic Acids Res. 44(D1):D110-5. doi: 10.1093/nar/gkv1176. URL: http://jaspar.genereg.net/

Matthews, B. H. C. (1931). The response of a muscle spindle during active contraction of a muscle. $J$ Physiol 72(2):153-174. pmid: 16994203

Matthews, B. H. C. (1933). Nerve endings in mammalian muscle. J Physiol. 78(1):1-53. pmid: 16994401

Matthews, P. B. (1962) .The differentiation of two types of fusimotor fibre by their effects on the dynamic response of muscle spindle primary endings. $Q J$ Exp Physiol Cogn Med Sci. 47:324-33. pmid: 13933877

Matthews, P. B. (1981). Evolving views on the internal operation and functional role of the muscle spindle. J Physiol. 320:1-30. pmid: 6459449

Murakoshi, H., and Trimmer, J. S. (1999) Identification of the Kv2.1 K+ channel as a major component of the delayed rectifier K+ current in rat hippocampal neurons. J Neurosci. 19(5):1728-35.

Muzumdar, M. D., Tasic, B., Miyamichi, K., Li, L., Luo, L. (2007). A global double-fluorescent Cre reporter mouse. Genesis 45, 593-605. doi: 10.1002/dvg.20335

McConnell, S. K. (1995). Strategies for the generation of neural diversity in the developing central nervous system. J Neurosci. 15(11):6987-98. pmid: 7472455

McGinnis W., and Krumlauf, R. (1992). Homeobox genes and axial patterning. Cell. 68(2):283302. pmid: 1346368 
McKenna. N. J., Lanz, R. B., and O'Malley, B. W. (1999). Nuclear receptor coregulators: cellular and molecular biology. Endocr Rev. 20(3):321-44. doi: 10.1210/edrv.20.3.0366

McVea, D. A., Donelan, J. M., Tachibana, A., Pearson, K. G. (2005). A role for hip position in initiating the swing-to-stance transition in walking cats. J Neurophysiol. 94(5): 3497-3508. doi: 10.1152/jn.00511.2005

Milburn, A. (1973a). The Early Development of Muscle Spindles in the Rat. J. Cell Sci. 12: 175-195.

Milburn, A. (1973b). The development of muscle spindles in the rat. Doctoral thesis, Durham University. http://etheses.dur.ac.uk/8583/

Milburn, A. (1984). Stages in the development of cat muscle spindles. J Embryol Exp Morphol. 82:177-216. pmid: 6238118

Miles, G. B., Dai, Y., and Brownstone, R. M. (2005). Mechanisms underlying the early phase of spike frequency adaptation in mouse spinal motor neurones. J. Physiol. 566(Pt 2), 519-532. doi:10.1113/jphysiol.2005.086033

Miles, G. B., Yohn, D. C., Wichterle, H., Jessell, T. M., Rafuse, V. F., Brownstone, R. M. (2004). Functional properties of motor neurons derived from mouse embryonic stem cells. J.Neurosci. 24(36), 7848-7858. doi: 10.1523/JNEUROSCI.1972-04.2004

Mitra, P., and Brownstone, R. M. (2012). An in vitro spinal cord slice preparation for recording from lumbar motor neurons of the adult mouse. J. Neurophysiol. 107(2), 728-741. doi:10.1152/jn.00558.2011

Mountcastle, V. B. (1997). The columnar organization of the neocortex. Brain. 120(Pt 4):701-22. pmid: 9153131

Muhr, J., Andersson, E., Persson, M., Jessell, T. M., Ericson, J. (2001). Groucho-mediated transcriptional repression establishes progenitor cell pattern and neuronal fate in the ventral neural tube. Cell. 104(6):861-73. pmid: 11290324

Mullen, R. J., Buck, C. R., and Smith, A. M. (1992). NeuN, a neural specific nuclear protein in vertebrates. Development. 116:201-211. pmid: 1483388

Müller, D., Cherukuri, P., Henningfeld, K., Poh, C. H., Wittler, L., Grote, P., Schlüter, O., Schmidt, J., Laborda, J., Bauer, S. R., Brownstone, R. M., Marquardt, T. (2014). Dlk1 promotes a fast motor neuron biophysical signature required for peak force execution. Science 343(6176), 12641266. doi:10.1126/science. 1246448

Muramoto, T., Mendelson, B., Phelan, K. D., Garcia-Rill, E., Skinner, R. D., Puskarich-May, C. (1996). Developmental changes in the effects of serotonin and N-methyl-D-aspartate on intrinsic biophysical properties of embryonic chick motor neurons. Neuroscience 75(2), 607-618. doi:10.1016/0306-4522(96)00185-6

Murthy, K. S. (1978). Vertebrate fusimotor neurones and their influences on motor behavior. Prog Neurobiol. 11(3-4):249-307. pmid: 154690

Nagao, M., Misawa, H., Kato, S., Hirai, S. (1998). Loss of cholinergic synapses on the spinal motor neurons of amyotrophic lateral sclerosis. J Neuropathol Exp Neurol. 57(4):329-33. pmid: 9600225

Nakanishi, S. T., and Whelan, P. J. (2010). Diversification of intrinsic motor neuron electrical properties during normal development and botulinum toxin-induced muscle paralysis in early postnatal mice. J. Neurophysiol. 103(5), 2833-2845.doi:10.1152/jn.00022.2010 
Nilsson, M., Markinhuhta, K. R., and Carlsson, M. L. (2006). Differential effects of classical neuroleptics and a newer generation antipsychotics on the MK-801 induced behavioural primitivization in mouse. Prog Neuropsychopharmacol Biol Psychiatry. 30(3):521-30. doi: 10.1016/j.pnpb.p..2005.11.010

Nolan, L. S., Maier, H., Hermans-Borgmeyer, I., Girotto, G., Ecob, R., Pirastu, N., Cadge, B. A., Hübner, C., Gasparini, P., Strachan, D. P., Davis, A., Dawson, S. J. (2013). Estrogen-related receptor gamma and hearing function: evidence of a role in humans and mice. Neurobiol Aging. 34(8):2077.e1-9. doi: 10.1016/j.neurobiolaging.2013.02.009.

Novitch, B. G., Chen, A. I., and Jessell, T. M. (2001). Coordinate regulation of motor neuron subtype identity and pan-neural properties by the bHLH repressor Olig2. Neuron. 31(5):773-89. pmid: 11567616

Oliveira, A. L., Hydling, F., Olsson, E., Shi, T., Edwards, R. H., Fujiyama, F., Kaneko, T., Hökfelt, T., Cullheim, S., Meister, B. (2003). Cellular localization of three vesicular glutamate transportermRNAs and proteins in rat spinal cord and dorsal root ganglia. Synapse. 50:117-129. doi: 10.1002/syn.10249

Ovcharenko, I., Nobrega, M. A., Loots, G. G., Stubbs, L. (2004). ECR Browser: a tool for visualizing and accessing data from comparisons of multiple vertebrate genomes. Nucleic Acids Res. 32(Web Server issue):W280-6. doi: 10.1093/nar/gkh355 URL: https://ecrbrowser.dcode.org

Padmowijoyo, D., and Yamadori, T. (1995). A Study on the Motor Control of the Extraocular Superior Oblique and Inferior Rectus Muscles in Albino Rats Using the Fluorescent Tracers Fluorogold and DiI (1,1'-Dioctadecyl-3,3,3',3'-Tetramethylindo-Carboyanine. Perchlorate). The Motor Control of Extraocular Muscles. Med. J. Indones. Vol 4, No 1, January-March, 1995.

Pearson, K. G. (2004). Generating the walking gait: role of sensory feedback. Prog Brain Res. 143:123-9. doi: 10.1016/S0079-6123(03)43012-4

Perret, C., and Berthoz, A. (1973). Evidence of static and dynamic fusimotor actions on the spindle responseto sinusoidal stretch during locomotor activity in the cat. Expl Brain Res. 18(2):178-188.

Perret, C., and Buser, P. (1972). Static and dynamic fusimotor activity during locomotor movements in the cat. Brain Res. 40(1):165-9. pmid: 4338260

Pettersson, K., Svensson, K., Mattsson, R., Carlsson, B., Ohlsson, R., Berkenstam, A. (1996). Expression of a novel member of estrogen response element-binding nuclear receptors is restricted to the early stages of chorion formation during mouse embryogenesis. Mech Dev. 54(2):211-23. pmid: 8652414

Philippidou, P., and Dasen, J. S. (2013). Hox genes: choreographers in neural development, architects of circuit organization. Neuron. 80(1):12-34. doi: 10.1016/ j.neuron.2013.09.020

Philippidou, P., Walsh, C. M., Aubin, J., Jeannotte, L., Dasen, J. S. (2012). Sustained Hox5 gene activity is required for respiratory motor neuron development. Nat. Neurosci. 15:1636-44. doi: $10.1038 / \mathrm{nn} .3242$

Pinter, M. J., Curtis, R. L., and Hosko, M. J. (1983). Voltage threshold and excitability among variously sized cat hindlimb motoneurons. J Neurophysiol. 50(3):644-57. pmid: 6311995

Placzek, M., Yamada, T., Tessier-Lavigne, M., Jessell, T., Dodd, J. (1991). Control of dorsoventral pattern in vertebrate neural development: induction and polarizing properties of the floor plate. Dev. Suppl. 2, 105-122. pmid: 1842349 
Poulet, B., de Souza, R., Knights, C. B., Gentry, C., Wilson, A. M., Bevan, S., Chang, Y. M., Pitsillides, A. A. (2014) Modifications of gait as predictors of natural osteoarthritis progression in STR/Ort mice. Arthritis Rheumatol. 66(7):1832-42. doi: 10.1002/art.38616

Powers, R. K., and Binder, M. D. (1985). Distribution of oligosynaptic group I input to the cat medial gastrocnemius motoneuron pool. J Neurophysiol. 53(2):497-517. pmid: 2984351

Pratt, W.B., and Toft, DO. (1997), Steroid receptor interactions with heat shock protein and immunophilin chaperones. Endocr Rev. 18(3):306-60. doi: 10.1210/ edrv.18.3.0303

Price, S. R., and Briscoe, J. (2004), The generation and diversification of spinal motor neurons: signals and responses. Mech Dev. 121(9):1103-15. doi: 10.1016/j.mod.2004.04.019

Prochazka, A. Proprioceptive feedback and movement regulation. In: Handbook of Physiology, Exercise-Regulation and Integration of Multiple Systems.2011. doi: 10.1002/cphy.cp120103

Prochazka, A., and Ellaway, P. (2012). Sensory systems in the control of movement. Compr Physiol. 2(4):2615-27.

Proske, U., and Gandevia, S. C. (2012). The proprioceptive senses: their roles in signaling body shape, body position and movement, and muscle force. Physiol Rev. 92(4):1651-97. doi: 10.1152/physrev.00048.2011.

Purves, D. "Neuroscience,” $3^{\text {rd }}$ ed., Sinauer Associates Inc., Sunderland. 2004.

R Core Team. (2015). R: A language and environment for statistical computing. R Foundation for Statistical Computing, Vienna, Austria. URL: http://www.R-project.org/.

Roelink, H., Augsburger, A., Heemskerk, J., Korzh, V., Norlin, S., Ruiz i Altaba, A., Tanabe, Y., Placzek, M., Edlund, T., Jessell, T. M. (1994). Floor plate and motor neuron induction by vhh-1, a vertebrate homolog of hedgehog expressed by the notochord. Cell. 76(4):761-75. pmid: 8124714

Romanes, G. J. (1941). The development and significance of the cell columns in the ventral horn of the cervical and upper thoracic spinal cord of the rabbit. $J$ Anat. 76(Pt 1):112-130.5. pmid: 17104877

Romanes, G. J. (1951). The motor cell columns of the lumbosacral spinal cord of the cat. J. comp. Neurol. 94, 313-364. J Comp Neurol. 94(2):313-63. pmid: 14832391

Romanes, G. J. (1964). The motor pools of the spinal cord. Prog Brain Res. 11:93-119. pmid: 14300484

Rossi, J., Balthasar, N., Olson, D., Scott, M., Berglund, E., Lee, C.E., Choi, M.J., Lauzon, D., Lowell, B. B., Elmquist, J. K. (2011). Melanocortin-4 receptors expressed by cholinergic neurons regulate energy balance and glucose homeostasis. Cell Metab. 13(2), 195-204. doi: 10.1016/j.cmet.2011.01.010

Rudy, B. (1988). Diversity and ubiquity of K channels. Neuroscience. 25(3):729-49. pmid: 2457185

Ruffini, A. (1898). On the Minute Anatomy of the Neuromuscular Spindles of the Cat, and on their Physiological Significance. J Physiol. 23(3):190-208.3. pmid: 16992453

Sambrook, J., and Russell, D. W. Molecular Cloning: A laboratory manual, $3^{\text {rd }}$ ed., Cold Spring Harbor Laboratory Press, New York. 2001. 
Sanders, T. A., Lumsden, A., and Ragsdale, C. W. (2002). Arcuate plan of chick midbrain development. J Neurosci. 22(24):10742-50. pmid: 12486167

Sanes, J. R., and Masland, R. H. (2015). The types of retinal ganglion cells: current status and implications for neural classification. Апnи Rev Neurosci. 38:221-46. doi: 10.1146/annurev-neuro071714-034120.

Santiago Ramón y Cajal. (1906). Nobel Lecture: The Structure and Connexions of Neurons. Nobelprize.org. Nobel Media AB 2014.

Sap, J., Munõz A., Schmitt, J., Stunnenberg, H., Vennström, B. (1989). Repression of transcription mediated at a thyroid hormone response element by the $\mathrm{v}$-erb-A oncogene product. Nature. 40(6230):242-4. doi: 10.1038/340242a0

Sato, Y., Kasai, T., Nakagawa, S., Tanabe, K., Watanabe, T., Kawakami, K., Takahashi, Y. (2007). Stable integration and conditional expression of electroporated transgenes in chicken embryos. Dev Biol. 305(2), 616-624. doi:10.1016/j.ydbio.2007.01.043

Sauer, B., and Henderson, N. (1988). Site-specific DNA recombination in mammalian cells by the Cre recombinase of bacteriophage P1. Proc Natl Acad Sci U S A. 85(14):5166-70. pmid: 2839833

Schwindt, P. C. (1973). Membrane-potential trajectories underlying motoneuron rhythmic firing at high rates. J. Neurophysiol., 36: 434-439.

Schwindt, P.C., and Calvin, W. H. (1972). Membrane potential trajectories between spikes underlying motoneuron rhythmic firing. J. Neurophysiol., 35: 311-325.

Schwindt, P. C., and Crill, W. E. (1981). Differential effects of TEA and cations on outward ionic currents of cat motoneurons. J Neurophysiol. 46(1):1-16. pmid: 6267208

Schwindt, P. C., and Crill, W. E. (1982) Factors influencing motoneuron rhythmic firing: results from a voltage-clamp study. J Neurophysiol. 48(4):875-90. doi: 10.1152/jn.1982.48.4.875

Schoultz, T. W., and Swett, J. E. (1972). The fine structure of the Golgi tendon organ. J Neurocytol. 1(1):1-26. pmid: 4668766

Shakespear's Hamlet. Ed. John Livingston Lowes. New York: Henry Holt and Co. 1914.

Shneider, N. A., Brown, M. N., Smith, C. A., Pickel, J., Alvarez, F. J. (2009). Gamma motor neurons express distinct genetic markers at birth and require muscle spindle-derived GDNF for postnatal survival. Neural Dev. 4:42. doi: 10.1186/1749-8104-4-42

Skoglund, S. (1960). Activity of muscle receptors in the kitten. Nature. 186:478-9. pmid: 13831506

Sonner, M. J., Walters, M. C., Ladle, D. R. (2017). Analysis of Proprioceptive Sensory Innervation of the Mouse Soleus: A Whole-Mount Muscle Approach. PLoS One. 12(1):e0170751. doi: 10.1371/journal.pone.0170751.

Spencer, R. F., and Porter, J. D. (2006). Biological organization of the extraocular muscles. Prog Brain Res. 151:43-80. doi: 10.1016/S0079-6123(05)51002-1

Stifani, N. (2014). Motor neurons and the generation of spinal motor neuron diversity. Front Cell Neurosci. 8:293. doi: 10.3389/fncel.2014.00293 
Szumski, A. J., Burg, D., Struppler, A., Velho, F. (1974). Activity of muscle spindles during muscle twitch and clonus in normal and spastic human subjects. Electroencephalogr Clin Neurophysiol. 37(6):589-97. pmid: 4140072

Takeoka, A., Vollenweider, I., Courtine, G., Arber, S. (2014). Muscle spindle feedback directs locomotor recovery and circuit reorganization after spinal cord injury. Cell. 159(7):1626-39. doi: 10.1016/j.cell.2014.11.019

Tanabe, Y., Roelink, H., and Jessell, T. M. (1995). Induction of motor neurons by Sonic hedgehog is independent of floor plate differentiation. Curr Biol. 5(6):651-8. pmid: 7552176

Tanabe, Y., William, C., and Jessell, T. M. (1998). Specification of motor neuron identity by the MNR2 homeodomain protein. Cell. 95(1):67-80. pmid: 9778248

Taylor, A. (1972). Muscle receptors in the control of voluntary movement. Paraplegia. 9(4):167-72. doi: $10.1038 / \mathrm{sc} .1971 .28$

Taylor, A., Durbaba, R., and Ellaway, P. H. (2004). Direct and indirect assessment of gammamotor firing patterns.Can J Physiol Pharmacol. 82(8-9):793-802. doi: 10.1139/y04-053

Taylor, A., Durbaba, R., Ellaway, P. H., Rawlinson, S. (2006). Static and dynamic gamma-motor output to ankle flexor muscles during locomotion in the decerebrate cat. $J$ Physiol. 571(Pt 3):711-23. doi: 10.1113/jphysiol.2005.101634

Tosolini, A. P., Mohan, R., and Morris, R. (2013). Targeting the full length of the motor end plates regions in the mouse forelimb increases the uptake of Fluoro-Gold into corresponding spinal cord motor neurons. Front Neurol. 20;4:58. doi: 10.3389/fneur.2013.00058

Tourtellotte, W. G., Keller-Peck, C., Milbrandt, J., Kucera, J. (2001). The transcription factor Egr3 modulates sensory axon-myotube interactions during muscle spindle morphogenesis. Dev Biol. 232(2):388-399. doi: 10.1006/dbio.2001.0202

Tourtellotte, W. G., and Milbrandt, J. (1998). Sensory ataxia and muscle spindle agenesis in mice lacking the transcription factor Egr3. Nat Genet. 20(1):87-91. doi: 10.1038/1757

Trainor, P. A., and Krumlauf, R. (2000). Patterning the cranial neural crest: Hindbrain segmentation and Hox gene plasticity. Nat. Rev. Neurosci. 1, 116-124. Nat Rev Neurosci. 1(2):11624. doi: $10.1038 / 35039056$

Tremblay, A. M., and Giguère V. (2007). The NR3B subgroup: an ovERRview. Nucl Recept Signal., 5:e009. doi: 10.1621/nrs.05009

Trichas, G., Begbie, J., and Srinivas, S. (2008). Use of the viral 2A peptide for bicistronic expression in transgenic mice. BMC Biol. 6:40. doi: 10.1186/1741-7007-6-40

Triezenberg, S. J., Kingsbury, R. C., and McKnight, S. L. (1988). Functional dissection of VP16, the trans-activator of herpes simplex virus immediate early gene expression. Genes Dev. 2(6):718-29. pmid: 2843425

Ugolini, G., Klam, F., Doldan Dans, M., Dubayle, D., Brandi, A. M., Büttner-Ennever, J., Graf, W. (2006). Horizontal eye movement networks in primates as revealed by retrograde transneuronal transfer of rabies virus: differences in monosynaptic input to "slow" and "fast" abducens motoneurons. J Comp Neurol. 498(6):762-85. doi: 10.1002/cne.21092

Underwood, E. (2015). The brain's identity crisis. Science. 349(6248):575-7. doi: $10.1126 /$ science. 349.6248 .575 
Vallbo, A. B. (1971). Muscle spindle response at the onset of isometric voluntary contractions in man. Time difference between fusimotor and skeletomotor effects. J Physiol. 218(2):405-31.

Vanderhorst, V. G., and Holstege, G. (1997). Organization of lumbosacral motoneural cell groups innervating hindlimb, pelvic floor, and axial muscles in the cat. J Comp Neurol. 382(1):46-76. pmid: 9136811

Wasicky, R., Horn, A. K., and Büttner-Ennever, J. A. (2004). Twitch and nontwitch motoneuron subgroups in the oculomotor nucleus of monkeys receive different afferent projections. $J$ Comp Neurol. 479(2):117-29. doi: 10.1002/cne.20296

Wessendorf, M. W. (1991). Fluoro-Gold: composition, and mechanism of uptake. Brain Res. 553(1):135-48. pmid: 1933270

Weir, J.P. (2001). Regulation of herpes simplex virus gene expression. Gene. 271(2):117-30. pmid: 11418233

Westbury, D. R. (1982). A comparison of the structures of alpha and gamma-spinal motoneurones of the cat. $J$ Physiol. 325:79-91. pmid: 7108785

Wherens, R. (2011). Chemometrics with R - Multivariate Data Analysis in the Natural Sciences and Life Sciences, Springer. doi:10.1007/978-3-642-17841-2

Willy, P. J., Murray, I. R., Qian, J., Busch, B. B., Stevens, W. C. Jr., Martin, R., Mohan, R,. Zhou, S., Ordentlich, P., Wei, P., Sapp, D. W., Horlick, R. A., Heyman, R. A., Schulman, I.G. (2004). Regulation of PPARgamma coactivator 1alpha (PGC-1alpha) signaling by an estrogen-related receptor alpha (ERRalpha) ligand. Proc Natl Acad Sci U S A. 101(24):8912-7.

Wolfram, V., Southall, T. D., Brand, A. H., Baines, R. A. (2012). The LIM-homeodomain protein islet dictates motor neuron electrical properties by regulating $\mathrm{K}(+)$ channel expression. Neuron. 75(4):663-74. doi: 10.1016/j.neuron.2012.06.015

Wu, Y., Wang, G., Scott, S. A., and Capecchi, M. R. (2008). Hoxc10 and Hoxd10 regulate mouse columnar, divisional and motor pool identity of lumbar motor neurons. Development 135:171-82. doi: $10.1242 /$ dev.009225

Wu, D., and Hersh, L. B. (1994). Choline acetyltransferase: celebrating its fiftieth year. $J$ Neurochem. 62(5):1653-63. pmid: 8158117

Yates, A., Akanni, W., Amode, M. R., Barrell, D., Billis, K., Carvalho-Silva, D., Cummins, C., Clapham, P., Fitzgerald, S., Gil, L., Girón, C. G., Gordon, L., Hr.lier, T., Hunt, S. E., Janacek, S. H., Johnson, N., Juettemann, T., Keenan, S., Lavidas, I, Martin, F. J., Maurel, T., McLaren, W, Murphy, D. N., Nag, R., Nuhn, M., Parker, A., Patricio, M., Pignatelli, M., Rahtz, M., Riat, H. S., Sheppard, D., Taylor, K., Thormann, A, Vullo, A., Wilder, S. P., Zadissa, A., Birney, E., Harrow, J., Muffato, M., Perry, E., Ruffier, M., Spudich, G., Trevanion, S. J., Cunningham, F., Aken, B.L., Zerbino, D. R., Flicek, P. (2015) Ensembl 2016. Nucleic Acids Res. 44(D1), D710D716. doi: 10.1093/nar/gkv1157

Yuengert, R., Hori, K., Kibodeaux, E.E., McClellan, J.X., Morales, J.E., Huang, T.W., Neul, J.L., Lai, H.C. (2015). Origin of a Non-Clarke's Column Division of the Dorsal Spinocerebellar Tract and the Role of Caudal Proprioceptive Neurons in Motor Function. Cell Rep. 13(6):1258-1271. doi: 10.1016/j.celrep.2015.09.064

Zelená, J. (1957). The morphogenetic influence of innervation on the ontogenetic development of muscle spindles. Journal of Embryology and Experimental Morphology. 5:283-292. 
Zelená, J. (1976). The role of sensory innervation in the development of mechanoreceptors. Prog Brain Res. 43:59-64. doi: 10.1016/S0079-6123(08)64338-1

Zelená, J., Soukup, T. (1973). Development of muscle spindles deprived of fusimotor innervation. $Z$ Zellforsch Mikrosk Anat. 144(3):435-52. pmid: 4362203

Zengel, J. E., Reid, S. A., Sypert, G. W., Munson, J. B. (1985). Membrane electrical properties and prediction of motor unit type of medial gastrocnemius motoneurons in the cat. J. Neurophysiol. 53:1323-1344. pmid: 3839011

Zong, H., Espinosa, J. S., Su, H. H., Muzumdar, M. D., Luo, L. (2005). Mosaic analysis with double markers in mice. Cell. 121(3), 479-492. doi: 10.1016/j.cell.2005.02.012

Zufferey, R., Donello, J. E., Trono, D., Hope, T. J. (1999). Woodchuck hepatitis virus posttranscriptional regulatory element enhances expression of transgenes delivered by retroviral vectors. J. Virol. 73(4), 2886-2892 (1999). pmid: 10074136 


\section{APPENDIX 1}

To verify that the anti-Err2 and anti-Err3 primary antibodies used in quantitative immunodetection studies were specific for mouse Err2 or mouse Err3 proteins, mouse Err2 and mouse Err3 genes inserted into Tol2 transposase system were expressed in chick MNs using in ovo electroporation technique (Materials \& Methods section 2.13.1). Chick neural tube cryosections were incubated with appropriate primary and secondary antibodies to check for cross-reactivity (Fig. 7.1). The studies showed that anti-Err2 primary antibody binds mouse Err2 protein, while anti-Err3 primary antibody binds to mouse Err3 protein. However, some fluorescent signal was detected on both electroporated and non-electroporated sides of spinal cord when chick cryosections were incubated with anti-Err3 primary and appropriate secondary antibodies, suggesting that anti-Err3 primary antibody may possibly bind endogenous chick Err3 protein or show some background fluorescence.

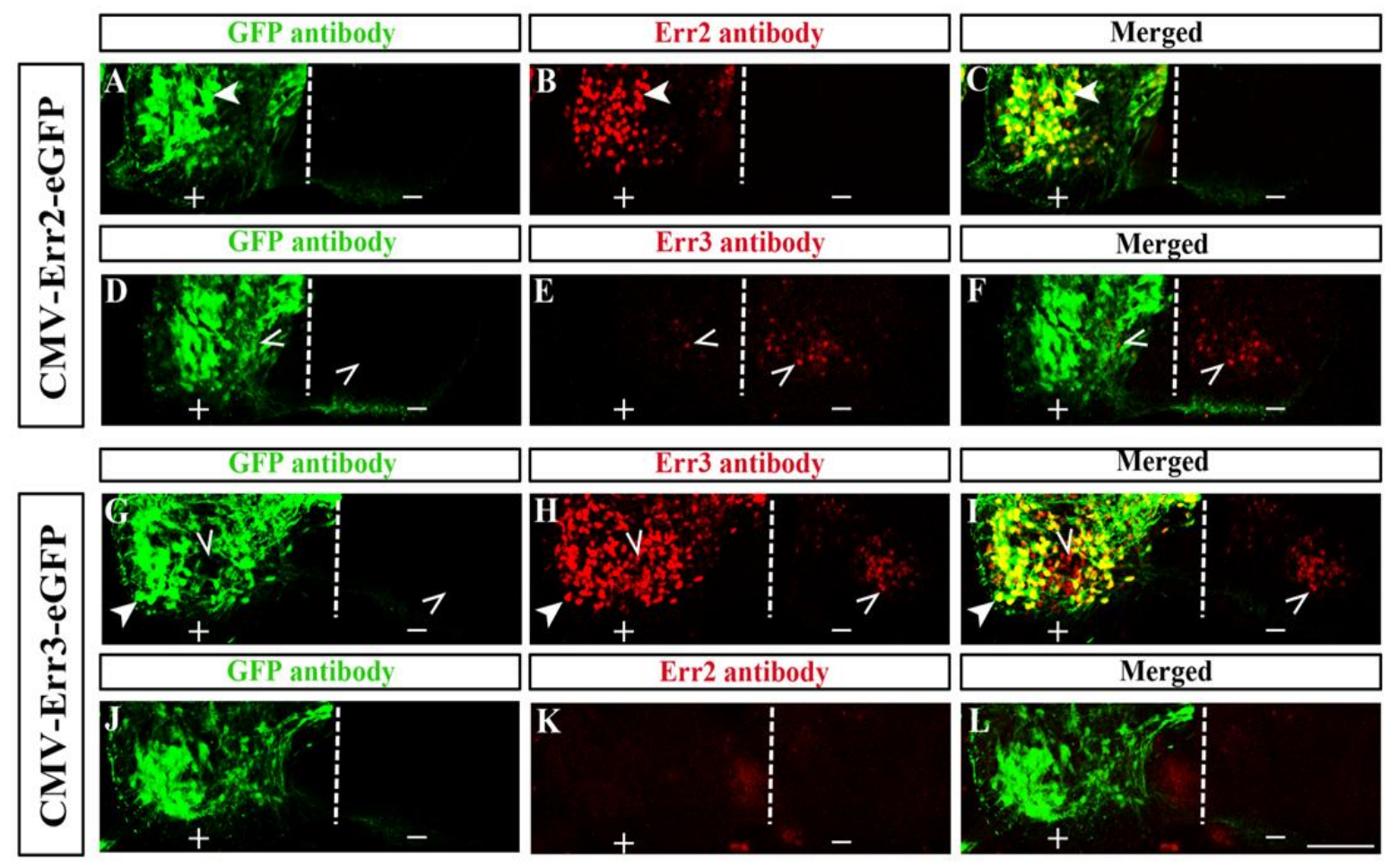

Figure 7.1 Anti-Err2 and anti-Err3 antibodies specifically recognize Err2 and Err3 proteins, respectively

(A-F) Split channel and merged confocal images of the ventral horn of the chick neural tube at E5.5 (HH St. 27-28) electroporated with a DNA construct carrying mouse Err2 (CMV-Err2-eGFP) and incubated with primary anti-Err2 antibody and anti-GFP antibody and appropriate secondary antibodies (A-C) or anti-Err3 and anti-GFP antibodies and appropriate secondary antibodies (D-F). (A-C) Solid arrows indicate co-localization of the anti-Err2 fluorescent signal with GFP fluorescent signal on the electroporated side (+). (D-F) While hollow arrows indicate endogenous fluorescent signal upon the incubation with the anti-Err3 antibody on both the electroporated (+) and the non- 
electroporated side (-) of the neural tube. (G-L) Confocal microscope images of chick embryo at E5.5 (HH St. 27-28) electroporated with a DNA construct carrying mouse Err3 (CMV-Err3-eGFP) and cryosections incubated with anti-Err3 and anti-GFP antibodies primary antibodies and appropriate secondary antibodies (G-I) or anti-Err2 and anti-GFP antibodies and appropriate secondary antibodies (J-L). (G-I) Solid arrows indicate co-localization of the anti-Err3 fluorescent signal with GFP fluorescent signal on the electroporated side $(+)$, while hollow arrows indicate endogenous signal on the non-electroporated side (-) of the neural tube. (J-L) No signal is detected by anti-Err2 antibody when electroporated with Err3 (CMV-Err3-eGFP) DNA construct. Scale bar represents $100 \mu \mathrm{m}$.

Wild-type mouse cryosections were used to verify whether Err2 and Err3 primary antibodies were bound by isotype-specific secondary antibodies (Fig. 7.2). The immunodetection studies showed that $\operatorname{IgG} 2 \mathrm{~b}$ and $\operatorname{IgG} 2 \mathrm{a}$ secondary antibodies are isotypespecific for Err2 and Err3 primary antibodies, respectively. Thus, anti-Err2 and anti-Err3 primary antibodies were co-incubated in a cryosection and treated with $\operatorname{IgG} 2 \mathrm{~b}$ and $\operatorname{IgG} 2 \mathrm{a}$ isotype-specific secondary antibodies to observe the co-expression patterns of Err2 and Err3 in mouse spinal cord postnatally.
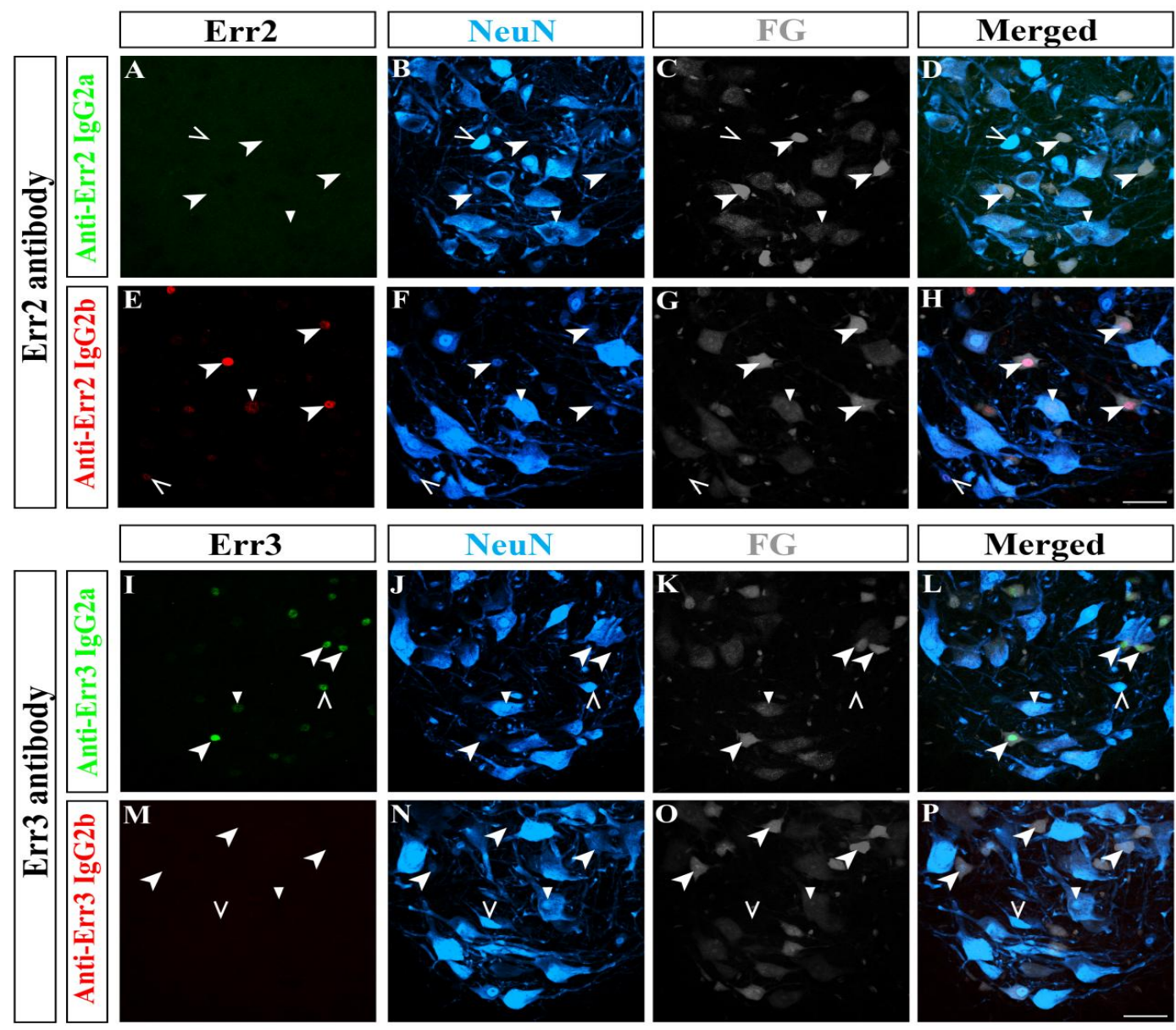

Figure 7.2 Secondary antibodies IgG2b and IgG2a are isotype-specific for Err2 and Err3, respectively

(A-P) Split channel and merged confocal images from P21 thoracolumbar spinal cord regions incubated with anti-Err2 or anti-Err3 primary antibodies and then with isotype-specific anti-Err2 
IgG2a or IgG2b and anti-Err3 IgG2a or IgG2b secondary antibodies, respectively. (A-D) Cryosections co-incubated with anti-Err2 primary antibody and anti-Err2 Alexa Fluor IgG2a secondary antibody showed no fluorescent signal in putative $\gamma$-MNs (solid arrows), putative $\alpha$-MNs (triangle), putative non-cholinergic cells (hollow arrows). (E-H) While cryosections co-incubated with anti-Err2 primary antibody and Alexa Fluor IgG2b secondary antibody showed high fluorescent signal (solid arrows) in putative $\gamma$-MNs. (I-L) Cryosections co-incubated with anti-Err3 primary antibody and anti-Err3 Alexa Fluor IgG2a secondary antibody showed fluorescent signal in putative $\gamma$-MNs (solid arrows). (M-P) While cryosections co-incubated with an anti-Err3 primary antibody and anti-Err3 Alexa Fluor IgG2b secondary antibody showed no signal in putative $\gamma$-MNs (solid arrows), putative $\alpha$-MNs (triangle), or putative non-cholinergic cells (hollow arrows). Scale bar represents $50 \mu \mathrm{m}$. 


\section{APPENDIX 2}

Table 8.1 Summary of biophysical properties of putative $\alpha$ - and $\gamma$-MNs from

\begin{tabular}{|c|c|c|c|c|}
\hline $\begin{array}{l}\text { Biophysical } \\
\text { Property } \\
\end{array}$ & $\begin{array}{l}\text { putative } \gamma \text {-MNs } \\
\text { (control) }(n=24)\end{array}$ & $\begin{array}{l}\text { putative } \alpha-M N s \\
\text { (control) }(n=22)\end{array}$ & $\begin{array}{l}\text { putative } \gamma \text {-MNs } \\
\left(E r r 2 / 3^{c K o}\right)(n=18)\end{array}$ & $\begin{array}{l}\text { putative } \alpha-M N s \\
\left(E r r 2 / 3^{c K o}\right)(n=9)\end{array}$ \\
\hline $\begin{array}{l}\text { mean input resistance } \\
{[\mathrm{M} \Omega]}\end{array}$ & $136.62 \pm 14.63$ & $\begin{array}{l}36.55 \pm 4.16 \\
a_{* * *}\end{array}$ & $\begin{array}{l}114.48 \pm 17.89 \\
b \text { N.S. }\end{array}$ & $\begin{array}{l}35.79 \pm 7.62 \\
c \text { N.S. }\end{array}$ \\
\hline mean capacitance $[\mathrm{pF}]$ & $76.07 \pm 6.01$ & $\begin{array}{l}239.1 \pm 17.17 \\
a_{* * *}\end{array}$ & $\begin{array}{l}117.67 \pm 8.93 \\
b_{* * *}\end{array}$ & $\begin{array}{l}247.85 \pm 22.19 \\
c_{\text {N.S. }}\end{array}$ \\
\hline mean rheobase $[\mathrm{pA}]$ & $221.87 \pm 31.34$ & $\begin{array}{l}909.09 \pm 82.11 \\
a_{* * *}\end{array}$ & $\begin{array}{l}419.72 \pm 84.11 \\
b_{*}\end{array}$ & $\begin{array}{l}855.55 \pm 124.85 \\
{ }^{c} \text { N.S. }\end{array}$ \\
\hline $\begin{array}{l}\text { mean AHP amplitude } \\
{[\mathrm{mV}]}\end{array}$ & $3.23 \pm 0.45$ & $\begin{array}{l}4.57 \pm 0.28 \\
a_{*}\end{array}$ & $\begin{array}{l}3.69 \pm 0.56 \\
b \text { N.S. }\end{array}$ & $\begin{array}{l}4.27 \pm 0.30 \\
c_{\text {N.S. }}\end{array}$ \\
\hline $\begin{array}{l}\text { mean AHP half-width } \\
\text { [ms] }\end{array}$ & $32.22 \pm 2.36$ & $\begin{array}{l}39.76 \pm 2.75 \\
a_{*}\end{array}$ & $\begin{array}{l}45.31 \pm 3.03 \\
b_{* * *}\end{array}$ & $\begin{array}{l}33.55 \pm 3.27 \\
c \text { N.S. }\end{array}$ \\
\hline $\begin{array}{l}\text { mean AHP half-decay } \\
{[\mathrm{ms}]}\end{array}$ & $22.10 \pm 1.89$ & $\begin{array}{l}27.78 \pm 1.94 \\
a_{*}\end{array}$ & $\begin{array}{l}30.08 \pm 2.50 \\
b\end{array}$ & $\begin{array}{l}23.89 \pm 2.55 \\
{ }^{c} \text { N.S. }\end{array}$ \\
\hline $\begin{array}{l}\text { mean firing frequency } \\
{[\mathrm{Hz}]}\end{array}$ & $50.65 \pm 3.23$ & $\begin{array}{l}20.41 \pm 1.70 \\
a_{* * *}\end{array}$ & $\begin{array}{l}27.61 \pm 3.15 \\
b_{* * *}\end{array}$ & $\begin{array}{l}25.03 \pm 3.76 \\
{ }^{c} \text { N.S. }\end{array}$ \\
\hline $\begin{array}{l}\text { mean firing frequency } \\
\text { (steady-state) }[\mathrm{Hz}]\end{array}$ & $49.37 \pm 3.20$ & $\begin{array}{l}20.18 \pm 1.32 \\
a_{* * *}\end{array}$ & $\begin{array}{l}26.25 \pm 3.10 \\
b_{* * *}\end{array}$ & $\begin{array}{l}24.99 \pm 2.42 \\
c \text { N.S. }\end{array}$ \\
\hline $\begin{array}{l}\text { mean firing } \\
\text { frequency } \\
\text { (instantaneous) [Hz] }\end{array}$ & $104.88 \pm 6.90$ & $\begin{array}{l}30.09 \pm 3.79 \\
a_{* * *}\end{array}$ & $\begin{array}{l}\mathbf{5 6 . 5 2} \pm 7.86 \\
b_{* * *}\end{array}$ & $\begin{array}{l}35.83 \pm 5.90 \\
c \text { N.S. }\end{array}$ \\
\hline mean gain $[\mathrm{Hz} / \mathrm{nA}]$ & $161.01 \pm 9.77$ & $\begin{array}{l}32.64 \pm 4.02 \\
a_{* * *}\end{array}$ & $\begin{array}{l}84.01 \pm 12.34 \\
b_{* * *}\end{array}$ & $\begin{array}{l}43.04 \pm 9.81 \\
{ }^{c} \text { N.S. }\end{array}$ \\
\hline $\begin{array}{l}\text { mean gain (steady- } \\
\text { state) }[\mathrm{Hz} / \mathrm{nA}]\end{array}$ & $155.12 \pm 9.87$ & $\begin{array}{l}31.66 \pm 3.57 \\
a_{* * *}\end{array}$ & $\begin{array}{l}78.01 \pm 12.25 \\
b_{* * *}\end{array}$ & $\begin{array}{l}41.86 \pm 8.81 \\
{ }^{c} \text { N.S. }\end{array}$ \\
\hline $\begin{array}{l}\text { mean gain } \\
\text { (instantaneous) } \\
{[\mathrm{Hz} / \mathbf{n A}]}\end{array}$ & $350.57 \pm 17.15$ & $\begin{array}{l}70.62 \pm 15.22 \\
a_{* * *}\end{array}$ & $b_{* * *}^{215.45 \pm 22.58}$ & $\begin{array}{l}79.15 \pm 23.97 \\
c \text { N.S. }\end{array}$ \\
\hline $\begin{array}{l}\text { MNs held at mean } \\
\text { membrane potential } \\
{[\mathrm{mV}]}\end{array}$ & $-69.74 \pm 0.23$ & $-68.70 \pm 0.20$ & $-69.43 \pm 0.35$ & $-68.53 \pm 0.73$ \\
\hline
\end{tabular}

Values show mean \pm standard error of the mean (S.E.M.).

${ }^{a}$ indicates significant difference between the putative $\gamma$-MNs (control) $(\mathrm{n}=24)$ and the putative $\alpha$-MNs (control) (n=22) (student's t-test);

${ }^{b}$ indicates significant difference between the putative $\gamma$-MNs (control) $(\mathrm{n}=24)$ and the putative $\gamma$-MNs $\left(E r r 2 / 3^{c K O}\right)(\mathrm{n}=18)$ (student's t-test); 
${ }^{c}$ indicates significant difference between the putative $\alpha$-MNs (control) $(\mathrm{n}=22)$ and the putative $\alpha$-MNs $\left(E r r 2 / 3^{c K O}\right)(\mathrm{n}=9)$ (student's t-test); $* * * p$-value $<0.001$;

$* * p$-value $<0.01$;

$* p$-value $<0.05$ 
Table 8.2 Summary of biophysical properties of chick spinal cord MNs

\begin{tabular}{|c|c|c|c|c|c|c|c|}
\hline $\begin{array}{l}\text { Biophysical } \\
\text { Property }\end{array}$ & $\begin{array}{l}\text { CMV-eGFP } \\
(\mathrm{n}=21)\end{array}$ & $\begin{array}{c}C M V-V P 16- \\
\text { Err2-eGFP } \\
(\mathrm{n}=20)\end{array}$ & $\begin{array}{l}C M V- \\
\text { Err2-eGFP } \\
(\mathrm{n}=21)\end{array}$ & $\begin{array}{c}C M V-E r r 3- \\
\quad e G F P \\
(\mathrm{n}=21)\end{array}$ & $\begin{array}{c}\text { CMV-EnR- } \\
\text { Err2-eGFP } \\
(\mathrm{n}=17)\end{array}$ & $\begin{array}{l}\dagger C M V- \\
e G F P \\
(\mathrm{n}=23)\end{array}$ & $\begin{array}{l}+C M V- \\
\text { Kcna10- } \\
e G F P \\
(\mathrm{n}=24)\end{array}$ \\
\hline $\begin{array}{l}\text { mean input } \\
\text { resistance }[\mathrm{M} \Omega]\end{array}$ & $\begin{array}{l}272.13 \pm \\
34.62\end{array}$ & $\begin{array}{l}298.72 \pm \\
26.67 \\
{ }^{a} \text { N.S. }\end{array}$ & $\begin{array}{l}309.96 \pm \\
28.96 \\
a_{\text {N.S. }}\end{array}$ & $\begin{array}{l}299.52 \pm \\
31.54 \\
a \text { N.S. }\end{array}$ & $\begin{array}{l}181.48 \pm \\
14.14 \\
a_{*}\end{array}$ & $\begin{array}{l}263.26 \pm \\
31.59 \\
b \text { N.S. }\end{array}$ & $\begin{array}{l}373.99 \pm \\
47.38 \\
c \text { N.S. }\end{array}$ \\
\hline $\begin{array}{l}\text { mean capacitance } \\
{[\mathrm{pF}]}\end{array}$ & $\begin{array}{l}237.77 \pm \\
14.82\end{array}$ & $\begin{array}{l}141.63 \pm \\
7.38 \\
a_{* * *}\end{array}$ & $\begin{array}{l}185.20 \pm \\
7.96 \\
a_{* * *}\end{array}$ & $\begin{array}{l}189.54 \pm \\
7.33 \\
a_{* * *}\end{array}$ & $\begin{array}{l}218.26 \pm \\
8.91 \\
a^{\text {N.S. }}\end{array}$ & $\begin{array}{l}241.29 \pm \\
12.01 \\
b \text { N.S. }\end{array}$ & $\begin{array}{l}183.96 \pm 9.74 \\
c_{* * *}\end{array}$ \\
\hline mean rheobase $[\mathrm{pA}]$ & $\begin{array}{l}160.95 \pm \\
25.85\end{array}$ & $\begin{array}{l}61 \pm 6.36 \\
a_{* * *}\end{array}$ & $\begin{array}{l}114.28 \pm \\
15.10 \\
{ }^{a} \text { N.S. }\end{array}$ & $\begin{array}{l}129.28 \pm \\
23.41 \\
{ }^{a} \text { N.S. }\end{array}$ & $\begin{array}{l}182.94 \pm \\
18.81 \\
a_{\text {N.S. }}\end{array}$ & $\begin{array}{l}160.43 \pm \\
19.97 \\
b \text { N.S. }\end{array}$ & $\begin{array}{l}67.91 \pm 11.15 \\
c_{* * *}\end{array}$ \\
\hline $\begin{array}{l}\text { mean AHP } \\
\text { amplitude [mV] }\end{array}$ & $8.48 \pm 0.75$ & $\begin{array}{l}5.85 \pm 0.51 \\
a_{* * *}\end{array}$ & $\begin{array}{l}6.54 \pm 0.81 \\
a^{a} \text { N.S. }\end{array}$ & $\begin{array}{l}5.44 \pm 0.51 \\
a_{* * *}\end{array}$ & $\begin{array}{l}8.17 \pm 0.61 \\
a \text { N.S. }\end{array}$ & $\begin{array}{l}7.52 \pm 0.58 \\
b \text { N.S. }\end{array}$ & $\begin{array}{l}6.33 \pm 0.54 \\
{ }^{c} \text { N.S. }\end{array}$ \\
\hline $\begin{array}{l}\text { mean AHP half- } \\
\text { width [ms] }\end{array}$ & $\begin{array}{l}118.53 \pm \\
9.37\end{array}$ & $\begin{array}{l}97.27 \pm 5.57 \\
{ }^{a} \text { N.S. }\end{array}$ & $\begin{array}{l}110.39 \pm \\
7.13 \\
{ }^{a} \text { N.S. }\end{array}$ & $\begin{array}{l}110.62 \pm \\
4.01 \\
{ }^{a} \text { N.S. }\end{array}$ & $\begin{array}{l}94.80 \pm 4.06 \\
a_{*}\end{array}$ & $\begin{array}{l}130.96 \pm \\
8.22 \\
b \text { N.S. }\end{array}$ & $\begin{array}{l}121.55 \pm 8.14 \\
{ }^{c} \text { N.S. }\end{array}$ \\
\hline $\begin{array}{l}\text { mean AHP half- } \\
\text { decay [ms] }\end{array}$ & $85.97 \pm 7.16$ & $\begin{array}{l}69.91 \pm 4.26 \\
{ }^{a} \text { N.S. }\end{array}$ & $\begin{array}{l}75.60 \pm \\
5.54 \\
a \text { N.S. }\end{array}$ & $\begin{array}{l}77.0 \pm 3.22 \\
{ }^{a} \text { N.S. }\end{array}$ & $\begin{array}{l}67.49 \pm 3.12 \\
a_{*}\end{array}$ & $\begin{array}{l}93.34 \pm 5.88 \\
b^{b} \text { N.S. }\end{array}$ & $\begin{array}{l}87.34 \pm 6.17 \\
{ }^{c} \text { N.S. }\end{array}$ \\
\hline $\begin{array}{l}\text { mean firing } \\
\text { frequency }[\mathrm{Hz}]\end{array}$ & $13.63 \pm 0.63$ & $\begin{array}{l}19.82 \pm 1.10 \\
a_{* * *}\end{array}$ & $\begin{array}{l}15.91 \pm 0.78 \\
a_{*}\end{array}$ & $\begin{array}{l}16.93 \pm 1.12 \\
a_{* * *}\end{array}$ & $\begin{array}{l}15.08 \pm 0.87 \\
{ }^{a} \text { N.S. }\end{array}$ & $\begin{array}{l}12.69 \pm 0.73 \\
{ }^{b} \text { N.S. }\end{array}$ & $\begin{array}{l}15.83 \pm 0.57 \\
c_{* * *}\end{array}$ \\
\hline $\begin{array}{l}\text { mean firing } \\
\text { frequency } \\
\text { (steady-state) [Hz] }\end{array}$ & $13.26 \pm 0.62$ & $\begin{array}{l}19.14 \pm 1.06 \\
a_{* * *}\end{array}$ & $\begin{array}{l}15.66 \pm \\
1.05 \\
a_{*}\end{array}$ & $\begin{array}{l}16.65 \pm 0.67 \\
a_{* * *}\end{array}$ & $\begin{array}{l}14.42 \pm 0.87 \\
{ }^{a} \text { N.S. }\end{array}$ & $\begin{array}{l}12.51 \pm 0.73 \\
{ }^{b} \text { N.S. }\end{array}$ & $\begin{array}{l}15.49 \pm 0.56 \\
c_{* * *}\end{array}$ \\
\hline $\begin{array}{l}\text { mean firing } \\
\text { frequency } \\
\text { (instantaneous) } \\
{[\mathrm{Hz}]}\end{array}$ & $14.87 \pm 1.05$ & $\begin{array}{l}32.01 \pm 2.36 \\
a_{* * *}\end{array}$ & $\begin{array}{l}18.39 \pm \\
1.42 \\
a_{*}\end{array}$ & $\begin{array}{l}22.0 \pm 1.26 \\
a_{* * *}\end{array}$ & $\begin{array}{l}14.79 \pm 1.36 \\
{ }^{a} \text { N.S. }\end{array}$ & $\begin{array}{l}14.12 \pm 1.22 \\
{ }^{b} \text { N.S. }\end{array}$ & $\begin{array}{l}21.46 \pm 0.94 \\
c_{* * *}\end{array}$ \\
\hline mean gain $[\mathrm{Hz} / \mathrm{nA}]$ & $44.84 \pm 2.75$ & $\begin{array}{l}101.01 \pm \\
6.58 \\
a_{* * *}\end{array}$ & $\begin{array}{l}58.0 \pm 3.49 \\
a_{* * *}\end{array}$ & $\begin{array}{l}62.47 \pm 2.66 \\
a_{* * *}\end{array}$ & $\begin{array}{l}44.58 \pm 5.14 \\
{ }^{a} \text { N.S. }\end{array}$ & $\begin{array}{l}42.45 \pm 2.63 \\
b^{b} \text { N.S. }\end{array}$ & $\begin{array}{l}46.57 \pm 2.69 \\
c^{c} \text { N.S. }\end{array}$ \\
\hline $\begin{array}{l}\text { mean gain (steady- } \\
\text { state) }[\mathrm{Hz} / \mathbf{n A}]\end{array}$ & $44.35 \pm 2.79$ & $\begin{array}{l}93.21 \pm 6.13 \\
a_{* * *}\end{array}$ & $\begin{array}{l}57.12 \pm \\
4.33 \\
a_{* * *}\end{array}$ & $\begin{array}{l}60.75 \pm 2.69 \\
a_{* * *}\end{array}$ & $\begin{array}{l}43.99 \pm 5.43 \\
{ }^{a} \text { N.S. }\end{array}$ & $\begin{array}{l}42.1 \pm 2.63 \\
{ }^{b} \text { N.S. }\end{array}$ & $\begin{array}{l}44.26 \pm 2.76 \\
{ }^{c} \text { N.S. }\end{array}$ \\
\hline $\begin{array}{l}\text { mean gain } \\
\text { (instantaneous) } \\
{[\mathrm{Hz} / \mathrm{nA}]}\end{array}$ & $60.86 \pm 5.67$ & $\begin{array}{l}209.59 \pm \\
16.68 \\
a_{* * *}\end{array}$ & $\begin{array}{l}79.59 \pm 8.88 \\
a^{\text {N.S. }}\end{array}$ & $\begin{array}{l}106.75 \pm \\
8.27 \\
a_{* * *}\end{array}$ & $\begin{array}{l}54.67 \pm 8.99 \\
{ }^{a} \text { N.S. }\end{array}$ & $\begin{array}{l}58.07 \pm 5.20 \\
b \text { N.S. }\end{array}$ & $\begin{array}{l}85.72 \pm 5.36 \\
c_{* * *}\end{array}$ \\
\hline $\begin{array}{l}\text { MNs held at mean } \\
\text { membrane potential } \\
{[\mathrm{mV}]}\end{array}$ & $\begin{array}{l}-60.82 \pm \\
0.44\end{array}$ & $-59.64 \pm 0.51$ & $\begin{array}{l}-60.38 \pm \\
0.21\end{array}$ & $\begin{array}{l}-60.82 \pm \\
0.24\end{array}$ & $-60.13 \pm 0.27$ & $\begin{array}{l}-60.91 \pm \\
0.16\end{array}$ & $-60.88 \pm 0.28$ \\
\hline
\end{tabular}

CMV-eGFP (control) versus CMV-VP16-Err2-eGFP, CMV-Err2-eGFP, CMV-Err3-eGFP, CMVEnR-Err2-eGFP studies were carried out using same internal solution;

$\dagger$ CMV-eGFP (control) versus CMV-Kcnal0-eGFP studies were carried out using same internal solution.

Values show mean \pm standard error of the mean (S.E.M.).

${ }^{a}$ indicates significant difference compared to CMV-eGFP (student's t-test);

${ }^{b}$ indicates significant difference between CMV-eGFP and CMV-eGFP (student's t-test); 
${ }^{c}$ indicates significant difference between CMV-eGFP and CMV-Kcnal0-eGFP (student's t-test); $* * * p$-value $<0.001$

$* * p$-value $<0.01$

$* p$-value $<0.05$ 


\section{APPENDIX 3}

Table 9.1 Forelimb variables control versus $E r r 2 / 3^{c K O}$ at mice at all speeds

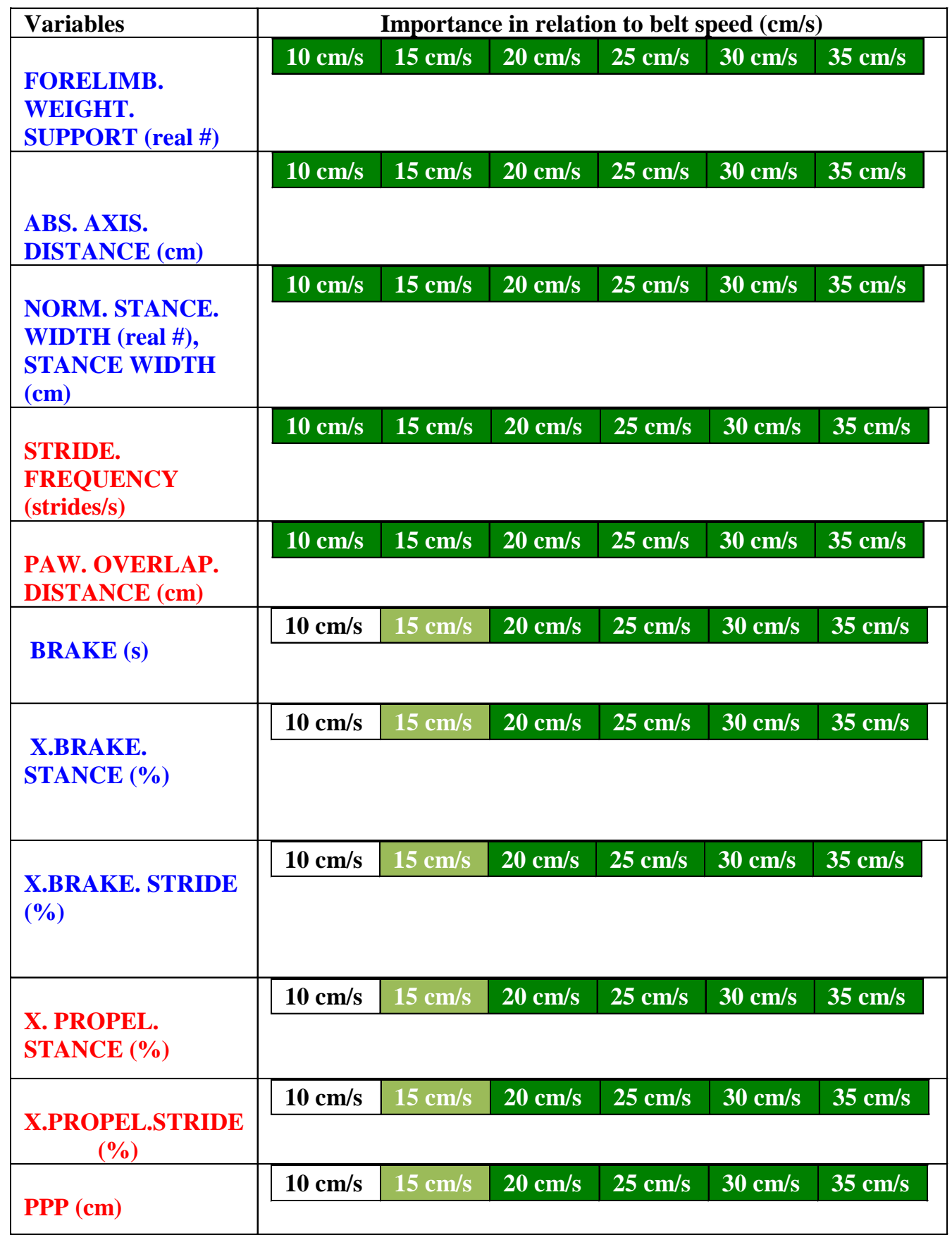




\begin{tabular}{|c|c|}
\hline \multirow{4}{*}{ color key } & mean Err $2 / 3^{\text {cko }}<$ mean control \\
\cline { 2 - 2 } & mean Err $2 / 3^{\text {cko }}>$ mean control \\
\cline { 2 - 2 } & more important at speed \\
\cline { 2 - 2 } & less important at speed \\
\hline
\end{tabular}


Table 9.2 Hindlimb variables control versus $E r r 2 / 3^{c K O}$ at mice at all speeds

\begin{tabular}{|c|c|c|c|c|c|c|}
\hline Variable & \multicolumn{6}{|c|}{ Importance in relation to belt speed $(\mathrm{cm} / \mathrm{s})$} \\
\hline MAX.DA.DT $\left(\mathrm{cm}^{2} / \mathrm{s}\right)$ & $10 \mathrm{~cm} / \mathrm{s}$ & $15 \mathrm{~cm} / \mathrm{s}$ & $20 \mathrm{~cm} / \mathrm{s}$ & $25 \mathrm{~cm} / \mathrm{s}$ & $30 \mathrm{~cm} / \mathrm{s}$ & $35 \mathrm{~cm} / \mathrm{s}$ \\
\hline PAW.AREA $\left(\mathrm{cm}^{2}\right)$ & $10 \mathrm{~cm} / \mathrm{s}$ & $15 \mathrm{~cm} / \mathrm{s}$ & $20 \mathrm{~cm} / \mathrm{s}$ & $25 \mathrm{~cm} / \mathrm{s}$ & $30 \mathrm{~cm} / \mathrm{s}$ & $35 \mathrm{~cm} / \mathrm{s}$ \\
\hline \multirow[b]{2}{*}{$\begin{array}{l}\text { X.PROPEL.STRIDE } \\
\text { (\%), PROPULSION } \\
\text { DURATION (ms) }\end{array}$} & $10 \mathrm{~cm} / \mathrm{s}$ & $15 \mathrm{~cm} / \mathrm{s}$ & $20 \mathrm{~cm} / \mathrm{s}$ & $25 \mathrm{~cm} / \mathrm{s}$ & $30 \mathrm{~cm} / \mathrm{s}$ & $35 \mathrm{~cm} / \mathrm{s}$ \\
\hline & & & & & & \\
\hline \multirow[b]{2}{*}{$\begin{array}{l}\text { X.STANCE.WIDTH } \\
\text { VARIANCE }(\mathbf{c m}), \\
\text { STANCE.WIDTH. } \\
\text { CV }(\text { CV } \%)\end{array}$} & $10 \mathrm{~cm} / \mathrm{s}$ & $15 \mathrm{~cm} / \mathrm{s}$ & $20 \mathrm{~cm} / \mathrm{s}$ & $25 \mathrm{~cm} / \mathrm{s}$ & $30 \mathrm{~cm} / \mathrm{s}$ & $35 \mathrm{~cm} / \mathrm{s}$ \\
\hline & & & & & & \\
\hline \multirow[b]{2}{*}{$\begin{array}{l}\text { ATAXIA.COEFFICI } \\
\text { ENT (real \#) }\end{array}$} & $10 \mathrm{~cm} / \mathrm{s}$ & $15 \mathrm{~cm} / \mathrm{s}$ & $20 \mathrm{~cm} / \mathrm{s}$ & $25 \mathrm{~cm} / \mathrm{s}$ & $30 \mathrm{~cm} / \mathrm{s}$ & $35 \mathrm{~cm} / \mathrm{s}$ \\
\hline & & & & & & \\
\hline \multirow{2}{*}{$\begin{array}{l}\text { PAW AREA } \\
\text { VARIANCE }\left(\mathrm{cm}^{2}\right)\end{array}$} & $10 \mathrm{~cm} / \mathrm{s}$ & $15 \mathrm{~cm} / \mathrm{s}$ & $20 \mathrm{~cm} / \mathrm{s}$ & $25 \mathrm{~cm} / \mathrm{s}$ & $30 \mathrm{~cm} / \mathrm{s}$ & $35 \mathrm{~cm} / \mathrm{s}$ \\
\hline & & & & & & \\
\hline \multirow{2}{*}{$\begin{array}{c}\text { X.BRAKE.STRIDE } \\
(\%)\end{array}$} & $10 \mathrm{~cm} / \mathrm{s}$ & $15 \mathrm{~cm} / \mathrm{s}$ & $20 \mathrm{~cm} / \mathrm{s}$ & $25 \mathrm{~cm} / \mathrm{s}$ & $30 \mathrm{~cm} / \mathrm{s}$ & $35 \mathrm{~cm} / \mathrm{s}$ \\
\hline & & & & & & \\
\hline \multirow{2}{*}{$\begin{array}{l}\text { X.BRAKE.STANCE } \\
(\%)\end{array}$} & $10 \mathrm{~cm} / \mathrm{s}$ & $15 \mathrm{~cm} / \mathrm{s}$ & $20 \mathrm{~cm} / \mathrm{s}$ & $25 \mathrm{~cm} / \mathrm{s}$ & $30 \mathrm{~cm} / \mathrm{s}$ & $35 \mathrm{~cm} / \mathrm{s}$ \\
\hline & & & & & & \\
\hline \multirow{2}{*}{$\begin{array}{l}\text { X.STANCE.STRIDE } \\
(\%)\end{array}$} & $10 \mathrm{~cm} / \mathrm{s}$ & $15 \mathrm{~cm} / \mathrm{s}$ & $20 \mathrm{~cm} / \mathrm{s}$ & $25 \mathrm{~cm} / \mathrm{s}$ & $30 \mathrm{~cm} / \mathrm{s}$ & $35 \mathrm{~cm} / \mathrm{s}$ \\
\hline & & & & & & \\
\hline \multirow{2}{*}{$\begin{array}{l}\text { MIDLINE.DISTANC } \\
\text { E (cm) }\end{array}$} & $10 \mathrm{~cm} / \mathrm{s}$ & $15 \mathrm{~cm} / \mathrm{s}$ & $20 \mathrm{~cm} / \mathrm{s}$ & $25 \mathrm{~cm} / \mathrm{s}$ & $30 \mathrm{~cm} / \mathrm{s}$ & $35 \mathrm{~cm} / \mathrm{s}$ \\
\hline & & & & & & \\
\hline \multirow[b]{2}{*}{$\begin{array}{l}\text { SHARED.STANCE } \\
(\mathrm{ms})\end{array}$} & $10 \mathrm{~cm} / \mathrm{s}$ & $15 \mathrm{~cm} / \mathrm{s}$ & $20 \mathrm{~cm} / \mathrm{s}$ & $25 \mathrm{~cm} / \mathrm{s}$ & $30 \mathrm{~cm} / \mathrm{s}$ & $35 \mathrm{~cm} / \mathrm{s}$ \\
\hline & & & & & & \\
\hline \multirow[b]{2}{*}{$\mathbf{P P P}(\mathbf{c m})$} & $10 \mathrm{~cm} / \mathrm{s}$ & $15 \mathrm{~cm} / \mathrm{s}$ & $20 \mathrm{~cm} / \mathrm{s}$ & $25 \mathrm{~cm} / \mathrm{s}$ & $30 \mathrm{~cm} / \mathrm{s}$ & $35 \mathrm{~cm} / \mathrm{s}$ \\
\hline & & & & & & \\
\hline
\end{tabular}




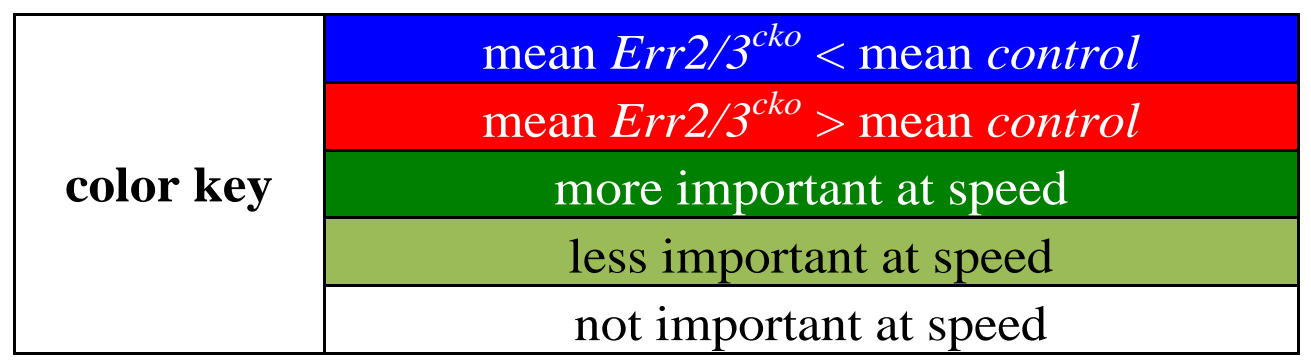

Statistical analysis using a Student's $t$-test gave a probability of $<0.1 \%$ of replicating the selected model's performance due to chance. The $\mathrm{Q}^{2}$ and RMSEP $p$-value are summarized for both forelimbs and hindlimbs for all Digigait treadmill speeds $(10 \mathrm{~cm} / \mathrm{s}, 15$ $\mathrm{cm} / \mathrm{s}, 20 \mathrm{~cm} / \mathrm{s}, 25 \mathrm{~cm} / \mathrm{s}, 30 \mathrm{~cm} / \mathrm{s}, 35 \mathrm{~cm} / \mathrm{s}$ ).

Table 9.3 $Q^{2}$ and RMSEP $p$-value for forelimbs and hindlimbs

\begin{tabular}{ccccc}
\hline & \multicolumn{2}{c}{ Forelimbs } & \multicolumn{2}{c}{ Hindlimbs } \\
\hline Belt Speed & $\mathrm{Q}^{2}$ & RMSEP & $\mathrm{Q}^{2}$ & RMSEP \\
$\mathbf{c m} / \mathbf{s}$ & p-value & $p$-value & p-value & $p$-value \\
$\mathbf{1 0}$ & $7.356 \mathrm{E}-28$ & $1.446 \mathrm{E}-36$ & $3.473 \mathrm{E}-24$ & $6.717 \mathrm{E}-42$ \\
$\mathbf{1 5}$ & $7.191 \mathrm{E}-26$ & $2.613 \mathrm{E}-35$ & $8.789 \mathrm{E}-25$ & $4.683 \mathrm{E}-37$ \\
$\mathbf{2 0}$ & $3.558 \mathrm{E}-30$ & $2.613 \mathrm{E}-41$ & $2.434 \mathrm{E}-29$ & $2.648 \mathrm{E}-38$ \\
$\mathbf{2 5}$ & $1.113 \mathrm{E}-30$ & $2.263 \mathrm{E}-36$ & $1.810 \mathrm{E}-33$ & $2.393 \mathrm{E}-38$ \\
$\mathbf{3 0}$ & $2.864 \mathrm{E}-26$ & $3.168 \mathrm{E}-28$ & $4.584 \mathrm{E}-34$ & $5.116 \mathrm{E}-44$ \\
$\mathbf{3 5}$ & $8.827 \mathrm{E}-33$ & $3.984 \mathrm{E}-29$ & $2.915 \mathrm{E}-39$ & $4.437 \mathrm{E}-43$ \\
\hline
\end{tabular}




\section{CURRICULUM VITAE}

\section{Personal Information:}

$\begin{array}{ll}\text { Name: } & \text { Mudassar Nazar Khan } \\ \text { Date of Birth: } & \text { November } 18^{\text {th }} 1984 \\ \text { Place of Birth: } & \text { Khanewal, Pakistan } \\ \text { Nationality: } & \text { American }\end{array}$

\section{Education:}

07/2013-Current Ph.D. in Biology (Developmental Neurobiology) at the Georg-AugustUniversität Göttingen

Thesis Project: "Regulatory mechanisms driving motor neuron functional diversification."

05/2010-05/2012 M.S. in Neuroscience at the University of Michigan, Ann Arbor Thesis Project: "The effects of Kir2.1 (inward-rectifier potassium channel) expression on neural morphology in the hippocampus in vivo."

08/2008-05/2010 M.S. in Biochemistry and Molecular Biology at The George George Washington University Thesis Project: "Ionizing radiation regulates p21-activated kinase activity and function."

09/2003-06/2007 B.S. in Cell Biology at the University of California, Santa Barbara 08/1999-05/2003 High School Diploma at Tokay High School, Lodi, California

\section{Publications:}

Johnson-Venkatesh EM, Khan MN, Murphy GG, Sutton MA, Umemori H. (2015). Excitability governs neural development in a hippocampal region-specific manner. Development. 142(22):3879-91. doi: 10.1242/dev.121202.

Li DQ, Ohshiro K, Khan MN, Kumar R. (2010). Requirement of MTA1 in ATR-mediated DNA damage checkpoint function. $J$ Biol Chem. 285(26):19802-12. doi: 10.1074/jbc.M109.085258.

Research Forum Publication: Yan A. Su, He Li, Ping He, Maree J. Webster, Qiuyang Zhang, Lei Zhang, Mudassar Khan, David M. Su, Robert J. Ursano and Owen M. Rennert. Identification of Stress-Responsive Genes, Canonical Pathways, Molecular Networks and Drug Targets in Brain Tissues of Human and Animals for Systems-Biology Study of PostTraumatic Stress Disorder. DOD Congressionally Directed Medical Research Programs. Military Health Research Forum 2009, Hallmark Crown Center Aug. 2009.

\section{Poster Presentation:}

Da-Qiang Li, Kazufumi Ohshiro, Rakesh Kumar. Requirement of MTA1 in ATR-mediated 
DNA Damage Checkpoint function. The George Washington University Research Day. (Washington, D.C. March 2010). Awarded $2^{\text {nd }}$ place.

\section{Awards:}

Rackham Merit Fellowship (RMF) at the University of Michigan, Ann Arbor (2010). "The Rackham Merit Fellowship is competitive and recognizes entering students who have outstanding academic qualifications, show exceptional potential for scholarly success in their graduate program, and demonstrate promise for contributing to wider academic, professional, or civic communities. The fellowship is a funding package that includes tuition, required fees, stipend and other benefits." Rackham Graduate School at the University of Michigan, Ann Arbor, 2010. 